PE NOTE: Some of the literature is very rare. As such, it has been impossible to determine details in some cases. With these references, missing information in indicated by ??.

\title{
THE THECAMOEBIAN BIBLIOGRAPHY
}

\section{F.S. Medioli, D.B. Scott, E. Collins, S. Asioli, and E.G. Reinhardt}

\section{ABSTRACT}

The literature on thecamoebians can be rather confusing, partly because it has been published in many different languages, but mainly because these Rhizopoda have the subject of study for a wide array of researchers with very different interests. Not only has this resulted in fragmentation of the literature due to research results being published in journals specializing in different fields, but inevitably has also resulted in development of a chaotic teminology and nomenclature. For example there is even confusion as to what to call the group, as terms such as "rhizopods", "testate amoebae" and "arcellaceans" have all been used by various authors as synonyms of "Thecamoebians". Even more confusing is the nomenclature of the described thecamoebian species. Lack of access to the literature and limited interchange between the various research groups, has generated many synonyms. Although only a first step this fairly complete bibliography on thecamoebians has been compiled to assist researchers become more aware of the available literature.

F.S. Medioli, D.B. Scott, E. Collins, Dalhousie University, Department of Earth Sciences, Halifax, Nova Scotia, B3H 3J5, Canada

S. Asioli, Istituto di Geologia Marina del CNR, via P. Gobetti 101, 40129 Bologna Italy

E.G. Reinhardt, McMaster University, School of Geography and Geology, Hamilton, Ontario, L8S 4M1, Canada

Keywords: arcellaceans, bibliography, rhizopods, testate amoebae, thecamoebians

Copyright: Paleontological Society, 15 March 1999

Submission: 22 September, 1998, Acceptance: 23 December 1998

\section{INTRODUCTION}

During the course of many years of research on thecamoebians we have built a substantial collection of reprints and photocopies of recent and old articles. As a result we have indirectly accumulated a large number of references (over 3000 ) which we report in this bibliography. We hope that this information will be a useful reference for researchers interested in working with fossil and sub-fossil thecamoebians. The World Wide Web is a perfect vehicle to disseminate this type of information, and makes it possible for constant updates and refinements to be made. 
The literature on thecamoebians can be rather confusing, partly because it is in many different languages but mainly because it is published in journals specialized in different fields. Inevitably, this has resulted in a somewhat chaotic teminology and nomenclature, so that terms such as "rhizopods", "testate amoebae" and "arcellaceans" have been used by various authors as synonyms of "Thecamoebians". The term "Arcellacea", often used as a synonym of Thecamoebians, is just a Superfamily of the Order Arcellinida, while "Thecamoebians" (Loeblich and Tappan, 1964) is an informal name, indicative of a polyphiletic [i.e.: coming from different Classes] "group" of convenience, including part of the class Rhizopodea, Subclass Lobosia, Orders Arcellinida, as well as part of the Class Reticularea, Subclass Filosia, Order Gromida [and probably part of the Suborder Allogromiina]. Even more confusing is the nomenclature of the thecamoebian species. Lack of access to the literature and poor communication between the various authors, as well as an almost endemic tendency to oversplit, have generated an almost unbelievable clutter of synonyms. We feel that the availability of a fairly complete bibliography, accessible to all researchers, will help mitigate some of these problems.

In forming this bibliography we tried to be as informative as possible. Some of the entries are incomplete and we were never able to find the actual article. We list them, however, because they still represent a clue and are thus potentially useful. We hope that by listing even the incomplete entries here for universal view on the WWW that we will finally complete them all, with the help of the users of this bibliography. In some cases we had a shortage of dependable information. In some casese we had a shortage of dependable information. The organization of many old journals, for example, can be very confusing. For example, the Learned Societies published the transcripts of oral presentation under two dates: the date of presentation and the date of publication of the journal. In addition, some authors were in the habit of presenting the same paper at meetings of different societies. It was sometimes frustrating for us to find, often after lengthy searches, that we had simply obtained a second copy of the same paper. To avoid similar struggles for users of this bibliography, we indicate when this is the case.

For some recent references, particularly European ones printed on local journals, we often had pre-prints with pagination different from the final one or no pagination at all; we could not do much about these. We apologize for the relatively few tentative or vague references, and for the abundant mistakes that we probably inherited from other authors' bibliographies. We feel they are better leads than no reference at all. Whenever we were aware of problems, however, we made it clear by inserting the symbol (?).

In a few cases, particularly amongst the very old references, our entries are "derived" from other authors' bibliographies and we have not seen the original published papers. Knowing that some earlier authors were at times somewhat inaccurate in their bibliographies, we do not rule out the possibility that some of our entries may lead nowhere. We definitely know that some of these authors did occasionally reference nonexistent papers (whether the references were simply wrong or whether they were quoting by memory articles that were never written, we do not know). 
For a a very few other references we were not certain whether the cited paper actually dealt with thecamoebians. Although the title and the general production of the authors suggest that the paper should be included here, without catually seeing the article we could not verify it. In such cases, we have supplied the reference anyway, in hopes that someone with access to the journal will let us know.

Since we intend to keep working at improving this bibliography, we would appreciate receiving corrections and clarifications and, if at all possible, reprints and/or photocopies of recent and old articles. Errors or ommissions can be brought to our attention at the email addresses listed with the Abstract. 


\section{AAA-AWE}

Abdul, K.S., Younis, W.R., 1984, Difflugia species from the sediments of the Mesopotamian region, southern Iraq: Journal of the Geological Society of Iraq. vs. 16-17, pp. 98-102.

Abraham, A., Biczok, F., Horvath, A., Megyeri, J., 1956, Hydrobiologische und faunistische studien im südwestlichen Teil des Bukk-Gebirges: Acta Biologica, v. 2, p. 137-154.

Agardh, C. A., 1827, Aufzählung einiger in den österreichischen Ländern gefundenen neue Gattungen und Arten von Algen: Flora oder allgemeine (Botanische Zeitschrift) (Regensburg), v. 10, no 2, p. 625-646.

Alabouvette, C., Coteaux, M. M., Old, K. M., Pussard, M., Reisinger, O., Toutain, F., 1981, Les Protozaires du sol: aspects écologiques et méthodologiques: L'Année biologique, v. 20 (fasc. 3), p. 255-303.

Allman, G.J., 1877a, Recent rersearches among some of the more simple sarcode organisms: Journal of the Linnean Society of London, v. 13, p. 261-305.

Allman, G.J., 1877b, Recent rersearches among some of the more simple sarcode organisms: Journal of the Linnean Society of London, v. 13, p. 385-439.

André, E., 1898, Note sur les Rhizopodes testacés du bassin de la Plessur: Jahrebericht der naturische Gesellschaft Graubünden, Neue Folge, v. 41, p. 57-59.

Archer, W., 1866a, "No title". (Remarks on freshwater Rhizopoda): Quarterly Journal of Microscopical Science, new ser., v. 6, p. 185-188.

Archer, W., 1866b, "No title". (Remarks on freshwater Rhizopoda): Quarterly Journal of Microscopical Science, new ser., v. 6, p. 266-267.

Archer, W., 1867a, "No title". (Remarks on freshwater Rhizopoda): Quarterly Journal of Microscopical Science, new ser., v. 7, p. 173-174.

Archer, W., 1867b, "No title". (Remarks on freshwater Rhizopoda): Quarterly Journal of Microscopical Science, new ser., v. 7, p. 177-178.

Archer, W., 1868a, "No title": Proceedings of the Dublin Microscopical Society, v. 1, no 2, p. 51-52.- Reproduces text of Archer 1866a.

Archer, W., 1868b, "No title": Proceedings of the Dublin Microscopical Society, v. 1, no 2, p. 118.- Reproduces text of Archer 1867b.

Archer, W., 1868c, Remarks on freshwater Rhizopoda: Quarterly Journal of Microscopical Science, new series, v. 8, p. 68-72. 
Archer, W., 1868d, "No title": Quarterly Journal of Microscopical Science, new series, v. 8, 295-296.

Archer, W., 1868e, "No title": Proceedings of the Dublin Microscopical Society, v. 1, no 2, p. 121-123.- Reproduces text of Archer 1867b.

Archer, W., 1869a, On some fresh-water Rhizopoda, new or little-known: Quarterly Journal of Microscopical Science, new series, v. 9, p. 250-271.

Archer, W., 1869b, On some fresh-water Rhizopoda, new or little-known: Quarterly Journal of Microscopical Science, new series, v. 9, p. 386-397, pls., 16, 17, 20.

Archer, W., 1869c, Remarks on freshwater Rhizopoda: Quarterly Journal of Microscopical Science, new series, v. 9, p. 321.

Archer, W., 1869d, "No title": Proceedings, Dublin Microscopical Society, Session 18th March, 1869: Quarterly Journal of Microscopical Science, new series, v. 9, p. 322.

Archer, W., 1869e, "No title": Quarterly Journal of Microscopical Science, new series, v. 9, p.421.

Archer, W., 1870a, On some fresh-water Rhizopoda, new or little known: Quarterly Journal of Microscopical Science, v. 10, p. 17-34, pls. 16, 17, 20 (contains the same plates as Archer 1869b).

Archer, W., 1870b, On some fresh-water Rhizopoda, new or little known: Quarterly Journal of Microscopical Science, v. 10, p. 101-124.

Archer, W., 1870c, On some freshwater Rhizopoda, new or little known: Proceedings of the Royal Irish Academy, ser. 2, Science Section, v. 1, p. 67-104, pls., 12, 13.Reprinted as Archer 1871b.

Archer, W., 1871a, A rhizopod new to Britain: Plagiophrys sphaerica: Quarterly Journal of Microscopical Science, new series, v. 11, p. 101.

Archer, W., 1871b, On some freshwater Rhizopoda, new or little known: Quarterly Journal of Microscopical Science, new series, v. 11, p. 107-151.

Archer, W., 1872a, Remarks on freshwater Rhizopoda: Quarterly Journal of Microscopical Science, new series, v. 12, p. 87-88.

Archer, W., 1872b, "No title": Quarterly Journal of Microscopical Science, new series, v. 12, p. 90.

Archer, W., 1872c, Remarks on freshwater Rhizopoda: Quarterly Journal of Microscopical Science, new series, v. 12, p. 194-195.

Archer, W., 1872d, Remarks on freshwater Rhizopoda: Quarterly Journal of Microscopical Science, new series, v. 12, p. 310. 
Archer, W., 1872e, "No title": Quarterly Journal of Microscopical Science, new series, v. 12, p. 423.

Archer, W., 1873a, Remarks on freshwater Rhizopoda: Quarterly Journal of Microscopical Science, new series, v. 13, p. 437.

Archer, W., 1873b, "No title": Proceedings of the Dublin Microscopical Club, v. 2, no 1, p. 108.

Archer, W., 1874a, Remarks on freshwater Rhizopoda: Quarterly Journal of Microscopical Science, new series, v. 14, p. 107-108.

Archer, W., 1875a, "No title": Proceedings of the Dublin Microscopical Club, v. 2, no 3, p. 301. (Same text as: Archer 1874a).

Archer, W., 1875b, "No title": Quarterly Journal of Microscopical Science, new series, v. 15, p. 202-203.

Archer, W., 1876a, Résumé of recent contributions to our knowledge of "Freshwater Rhizopoda." Part 1. Heliozoa: Quarterly Journal of Microscopical Science, new series, v. 16, p. 283-309.

Archer, W., 1876b, Résumé of recent contributions to our knowledge of "Freshwater Rhizopoda." Part 2. Heliozoa: Quarterly Journal of Microscopical Science, new series, v. 16, p. 347-376.

Archer, W., 1876c, Remarks on freshwater Rhizopoda: Quarterly Journal of Microscopical Science, new series, v. 16, p. 107-108.

Archer, W., 1876d, Remarks on freshwater Rhizopoda: Quarterly Journal of Microscopical Science, new series, v. 16, p. 237.

Archer, W., 1876e, Remarks on freshwater Rhizopoda: Quarterly Journal of Microscopical Science, new series, v. 16, p. 331.

Archer, W., 1876f, New species of Qudarula, Schulze: Quarterly Journal of Microscopical Science, new series, v. 16,p. 337.

Archer, W., $1876 \mathrm{~g}$, A new form of freshwater amphistomatous rhizopod: Quarterly Journal of Microscopical Science, new series, v. 16, p. 340-342.

Archer, W., 1876h, Proceedings of the Dublin Microscopical Club, 23rd March, 1876: Quarterly Journal of Microscopical Science, new series, v. 16, p. 343-344.

Archer, W., 1876i, "No title": Proceedings of the Dublin Microscopical Club, v. 3, no 1, p. 110-111.

Archer, W., 1877a, Résumé of recent contributions to our knowledge of "Freshwater Rhizopoda." Part 3. Heliozoa (Desmothoraca) and Monothalamia (Monostomata): 
Quarterly Journal of Microscopical Science, new series, v. 17, p. 67-80.

Archer, W., 1877b, Résumé of recent contributions to our knowledge of "Freshwater Rhizopoda." Part 4: Quarterly Journal of Microscopical Science, new series, v. 17, p. 107-124, pl. 8.

Archer, W., 1877c, Résumé of recent contributions to our knowledge of "Freshwater Rhizopoda." Part 4: Quarterly Journal of Microscopical Science, new series, v. 17, p. 197-204, pl. 13.

Archer, W., 1877d, Résumé of recent contributions to our knowledge of "Freshwater Rhizopoda." Part 4: Quarterly Journal of Microscopical Science, new series, v. 17, p. 330-353, pl. 21.

Archer, W., 1877e, Proceedings of the Dublin Microscopical Club, session July 13, 1876: Quarterly Journal of Microscopical Science, new series, v. 17, p. 102-104.

Archer, W., 1877f, "No title": Quarterly Journal of Microscopical Science, new series, v. 17, p. 194, pl. 12.

Archer, W., 1877g, "No title": Quarterly Journal of Microscopical Science, new series, v. 17, p. 196.

Archer, W., 1877h, Amphizonella violacea, Greeff: Quarterly Journal of Microscopical Science, new series, v. 17, p. 464.

Archer, W., 1877i, "No title": Quarterly Journal of Microscopical Science, new series, v. 17, p. 469.

Archer, W., 1878a, "No title": Proceedings of the Dublin Microscopical Club, v. 3, no 2, p. 228.

Archer, W., 1878b, "No title": Quarterly Journal of Microscopical Science, new series, v. 18, p. 104-105.

Archer, W., 1878c, "No title": Quarterly Journal of Microscopical Science, new series, v. 18, p. 212-213.

Archer, W., 1878d, "No title": Proceedings of the Dublin Microscopical Club, v. 3, no 2, p. 198.

Archer, W., 1880a, "No title": Proceedings of the Dublin Microscopical Club, v. 3, no 3, p. 273, pl. 10.

Archer, W., 1880b, "No title": Proceedings of the Dublin Microscopical Club, v. 3, no 3, p. 284-285.

Archer, W., 1880c, "No title": Proceedings of the Dublin Microscopical Club, v. 3, no 3, p. 311-312. 
Arndt, A., 1924, Rhizopodenstudien. I: Archiv für Protistenkunde, v. 49, no 1, p. 1-83, pl. 1-5.

Arndt, H., 1993, A critical review of the importance of rhizopods (naked and testate Amoebae) and actinopods (Heliozoa) in lake plankton: MAR.-MICROB.-FOOD-WEBS, v. 7 , pp. 3-29

Arnold, Z.M., 1966, Observations on the sexual generation of Gromia oviformis Dujardin: Journal of Protozoology, v. 13, no 1, p. 23-27.

Arnold, Z.M., 1971, Ecological relationships of Gromia oviformis, a large coastal sarcodinian (Protozoa): Abstract, National Coastal and Shallow Water Research Conference, v. 2, pp. 16.

Arnold, Z.M., 1982, Shell-wall lamination in Gromia oviformis Dujardin: Journal of Foraminiferal Research, v. 12, pp. 298-316.

Arnold, Z.M., McLendon, ??, 1952, Structure and paleontological significance of the oral apparatus of the foraminiferoid Gromia oviformis Dujardin: Journal of Paleontology, v. 26, pp. 829-831.

Asioli, A., 1993, Ricerche su paleoambienti olocenici di alcune aree padanoadriatiche attraverso l'individuazione di ecofenotipi nei Rizopodi: (Unpublished Ph. D. Thesis) Università degli Studi di Parma, 105 pp.

Asioli, A., Medioli, F.S., 1992, Ricostruzione dei paleoambienti attraverso le Tecamebe in alcuni laghi sudalpini (Orta, Varese e Candia): Atti X Congresso Associazione Italiana Oceanologia e Limnologia, (Alassio, 4-6 novembre 1992), p. 487-501.

Asioli, A., Medioli, F.S., Patterson, R.T., 1996, Thecamoebians as a tool for reconstruction of paleoenvironments in some Italian lakes in the foothills of the Southern Alps (Orta, Varese and Candia): Journal of Foraminiferal Research, v.26, no 3, p. 248-263.

Auerbach, L., 1856: Ueber die Einzelligkeit der Amoeben: Zeitschrift für wissenschaftliche Zoologie, v. 7, p. 365-430, pls. 19-22.

Averintsev, S., 1906, Rhizopoda prêsnkh vod. Imperatorskago Sankt Peterburgskago Obshchestva Estestvoisptateleì Trud, v. 36, no 2, p. 1-351, pls. 15 .

Averintsev, S., 1907, Fresh-water Rhizopods: Journal of the Royal Microscopical Society, p. 684; written report of a verbal presentation.

Awerintzew, S. (1906) Die Süsswasser Rhizopoden. I and II. Trudui S.-Peter. Obscch. 36, 1-351.

Awerintzew, S. (1907) Ueber einige neue Arten gehaüsetragender Rhizopoden des 
Süsswassers. Archiv für Protistenkunde, 8 86-94. 


\section{BAB-BEY}

Babitski, V. A., 1980a, Methodological problems in studies of shell amoebas Rhizopoda Testacea and their population development in the Naroch Group of Lakes Belorussian-SSR USSR: Vyestsi Akademii Navuk Bssr Syeryya Biyalahichnykh Navuk, v. 0, p. 109-114.

Babitski, V. A., 1980b, Micro benthos in three different types of lake: Gidrobiologicheskii Zhurnal, v. 16, p. 37-45.

Bailey, J. W., 1850. Microscopic observations made in South Carolina, Georgia and Florida. Smithsonian Contributions to Knowledge, v. 2, art. 8, p. 1-48, pls. 1-3.

Bailey, J. W., 1853, Observations on a newly discovered animacule: American Journal of Science and Arts, 2nd series, v. 15, 45, p. 341-347.

Bailey, J. W., date ??,A sketch of the Infusoria of the family Bacillaria, Part II: American Journal of Sciences and Arts, v. 42, p. 85-105, pls. 1, 2.

Bailey, J. W., date ??,Notice of microscopic forms found in the surroundings of the Sea of Kamtschatka: American Journal of Science and Arts, 2nd series, v. 22, 64, p. 1-6, pl. I.

Balbiani, E, G., 1884, Les organisms unicellaires. Les Protozoaires: Journal de Micrographie, v. 8, p. 9-375.

Balik, V., 1986, The study of Algerian Testacea Rhizopoda: Vestnik Ceskoslovenske Spolecnosti Zoologicke, v. 50, p. 81-86.

Balik, V., 1988: Centropyxis moldavica new species Rhizopoda Testacea a new species of soil Testacea from Czechoslovakia: Acta Protozoologica, v. 27, p. 161-164.

Bamforth, S.S., 1963, Microhabitat and community structure as ecological factors for Protozoa: in: Ludvík, J., Lom,J., and Vávra, J. (eds.): Progress in Protozoology: Proceedings of the First International Congress on Protozoology, Prague, August 1961, p.301-302, Publishing House of the Czechoslovak Academy of Sciences, Prague.

Bamforth, S.S., 1971, The numbers and proportions of Testacea and Ciliates in litters and soils: Journal of Protozoology, v. 18, 1, p. 24-28.

Bamforth, S.S., 1976, Rhizosphere-soil microbial comparisions in subtropical forests of Southeastern Louisiana: Transactions of the American Microscopical Society, v. 95, p. 613-621.

Bamforth, S.S., 1977, Litters and soils as freshwater ecosystems: in: Cairns, J. J., (ed.): Aquatic microbial communities, p. 243-256, Garland Publishing Inc.: "Garland Reference Library of Science and Technology", v. 15, New York. 
Bamforth, S.S., 1980a, Terrestrial Protozoa: Journal of Protozoology, v. 27, 1, p. 3336.

Bamforth, S.S., de Pelaez, N., 1977, Numbers and proportions of microorganisms in humid forest litters: Proceedings of the Louisiana Academy of Sciences, v. 40, p. 33-38.

Barbieri, S.M., Orlandi, J.L.G., 1989, Ecological studies on the planktonic Protozoa of a eutrophic reservoir (Rio Grande Reservoir, Brazil): Hydrobiologia, v. 183, pp. 1-10

Bargoni, E., 1894, Di un foraminifero parassita nelle Salpe (Salpicola amylacea , n. g., n. sp.) e considerazioni sui corpuscoli amilacei dei Protozoi superiori: Ricerche fatte nel Laboratorie di Anatomia normale della R. Università di Roma, v. 4, fasc. 1-2, p. 43-64, pl. 3, 4.

Barker, J., 1868, Proceedings of the Dublin Microscopical Club, December 19, 1867: Quarterly Journal of Microscopical Science, new series, v. 8, p.123.

Barker, J., 1869, Brief notes on freshwater Rhizopods: Quarterly Journal of Microscopical Science, new series, vs. 6-9, pages unknown.

Barker, T., 1992, A scanning electron microscope study of Arcellaceans from Oromocto Lake, New Brunswick, Department of Earth Sciences, Carleton University Honour's Thesis, $34 \mathrm{p}$.

Barnard, W. S., 1876, Protozoan studies: American Association for the Advancement of Science, Proceedings, v. 24 (1875), p. 240-242.

Barnard, W. S., 1879, New rhizopods: American Quarterly Microscopical Journal, v. 1, 2, p. 83-85, pl. 8.

Barrois, T., 1888, Faune d'eau douce des Açores, II \& III: - no further information; this appears to be a book.

Barrois, T., 1894, Contribution à l'ètude de quelques Lacs de Syrie: Revue Biologique du Nord de la France, v. 6, p. 224-312.

Barrois, T., 1896, Recherches sur la faune des eaux douces des Açores: Mémoires de la Société des Science de l'Agricolture et des Arts de Lille, ser. 5, v. 6, p. 1-172, pls. 1-3.

Bartlett, G.A., 1966, Distribution and abundance of Foraminifera and Thecamoebina in Mirimachi River and Bay: Bedford Institute of Oceanography, Report \# 66-2, 104 pp.

Bartos, E., 1938a, Die moosbewohnenden Rhizopoden III, die Vertreter der Familie Difflugiidae: Priroda, v.31, - pages unknown.

Bartos, E., 1938b, Eine neue moosbewohnende Nebella-Art, Nebela pulchra m. n. sp: Archiv für Protistenkunde, v. 90, no 2, p. 346-347. 
Bartos, E., 1938c, Beitrage zur kenntnis des Baues der Schale von Bullinula indica Penard: Archiv für Protistenkunde, v. 90, no 2, p. 348-353.

Bartos, E., 1938d, Die moosbewohnenden Rhizopoden IV, die Vertreter der Familie Nebelidaedae: Priroda, v.31, - pages unknown.

Bartos, E., 1938e, Zastipci celedi Difflugiidae: Casopi su Priroda, v. 31, no 2, - pages unknown.

Bartos, E., 1938f, Zastupci celedi Nebellidae: Casopi su Priroda, v. 31, no 2,- pages unknown.

Bartos, E., 1939, Über den Baunder Shalen einigar moosbewohnender Rhizopoden: Archiv für Protistenkunde, v. 92, p. 149-151.

Bartos, E., 1940a, Studien über die moosbewohnenden Rhizopoden der Karpaten: Archiv für Protistenkunde, v. 94, p. 93-160.

Bartos, E., 1940b, Zastupci celedi Arcellidae et Gromidae: Casopi su Priroda, - other data unknown.

Bartos, E., 1946, The analysis of the microscopical fauna of the Bohemian mosses: Vestnik Ceskoslovenske Spolecnosti Zoologicke, v. 10, p. 55-88.

Bartos, E., 1947, Korenonozci mech prazkeho okoli (The moss dwelling Rhisopoda of the neighbourhood of Prague): Z. Sborník Klunu prírodovédeckého v Breb, v. 28, p. 1-10.

Bartos, E., 1949a, Drobnohladna fauna slovenskych machov: Académie scienturium et artium slovaca, - other data unknown.

Bartos, E., 1949b, Mikroskopich a zvirena su'uavskych mechu. Mechy okoli Plesného jereza: Vestnik Csl. zoologické spotecnosti, v. 13, p. 10-29.

Bartos, E., 1963a, Die Rhizopoden einiger Moosproben aus China: Acta Societatis Zoologicae Bohemoslovenica, v. 27, no 2, p. 85-96.

Bartos, E., 1963b, Rhizopoden einiger moosproben aus Java: Acta Universitatis Carolinaae, Biologica, p. 119-190.

Bartos, E., Korenonozci virnici a zelvusky mechu Sumavskych Predhori. - other data unknown

Bartos, E., date ??, Zvirena beskydskych aerophytickych mechu. - other data unknown

Baruah, A., Singh, D.K., Sinha, A.K., Sharma, U.P., 1993, Plankton variability of a tropical wetland, Kawar Lake (Begusarai), Bihar: Journal of Freshwater Biology, v. 5, pp. 27-32 
Baumeister, W., 1975, Ein bemerkenswerter Moortümpel bie Gschöd, Gemeinde Wittibreut (Niederbayern): Mitteilungen der Zoologischen Gesellschaft in Braunau, v. 2, p. 171-183.

Baumeister, W., 1976, Der Kreis Rottal-Inn als hydrobiologisches Forschungsgebiet: Arbeitstälte zur Erforschung des Lebens in Kleingewässern, Mitteilung, v. 10, p. 115.

Beardsley, A. E., 1902, Notes on Colorada Protozoa: Transactions of the American Microscopical Society, v. 23, p. 49-58, pl. XI.

Bennet, L. W., Bamforth, S. S., 1982, Seasonal distribution of litter soil ciliates and testacea in a cold Sagebrush Desert: 97th Annual Meeting of the American Microscopical Society, Dallas, Texas, USA, Dec. 27-30, 1981: Transactions of the American Microscopical Society, v. 101, p. 112.

Bereczky, M. C., 1969, Untersuchungen über die Protozoenfauna der Donau bei Alsogöd (Ungarn): (Danubialia Hungarica, LII): Opuscola Zoologica (Budapest), v.9, no I, p. 87-96, pls. 1-3.

Bereczky, M. C., 1970, Untersuchungen über die Rhizopodenfauna der Aggteleker "Baradla" - Höhle. (Biospeologica Hungarica, XXXII): Opuscula Zoologica (Budapest), v. 10,1, p. $69-82$.

Bereczky, M. C., 1973a, Kennzeichnung des Schlammes im offenen Wasser des Balatons mit Hilfe des Testaceen-Fauna: Verhandlungen des Internationalen Vereins für Limnologie, v. 18, p. 1406-1412.

Bereczky, M. C., 1973b, Beitrage zur kenntnis der im Eprofundal des Balaton lebenden testaceen: Annales Universitatis Scientiarum Budapestiensis de Rolando Eötvös Nominatae (Sectio biologica), v. 15, p. 117-127.

Bereczky, M. C., 1977, Kennzeichnung der saprobiologischen Verhältnisse des oberen ungarischen Donauabschnittes mit Hilfe von Protozoen als Indikatoren (Danubialia Hungarica, XLIV): Opuscula zoologica (Budapest), v. 14, p. 55-66.

Bereczky, M. C., 1979a, Gestaltung der Ciliata- und Testacea- Populationen der Donau unter der Einwirkung des Flussregimes und der Wasserkunstbauten zwischen Vác und Göd: Annales Universitatis Scientiarum Budapestiensis de Rolando Eötvös Nominatae (Sectio biologica), v. 20-21, p. 205-227.

Bereczky, M. C., 1979b, Vergeichende Untersuchungen über die Gestaltung der im Plankton vorkommenden Testaceen im Haupt und Nebenarm der Donau bei Göd (Stromkm 1669): Annales Universitatis Scientiarum Budapestiensis de Rolando Eötvös Nominatae (Sectio biologica), v. 20-21, p. 229-236.

Berger, H., Foissner, W., Adam, H., 1984, Effects of ammotium sulfate on soil Testacea Ciliates and Nematodes: Annual Meeting of the Society of Protozoologists 
(German Section), Bonn, West Germany, Mar. 29-31, 1984: Journal of Protozoology, v. 31, p. 59

Berger, H., Foissner, W., Adam, H., 1986, Field experiments on the affects of fertilizers and lime on the soil microfauna of an alpine pasture: Pedobiologia, v. 29, p. 261-272.

Bergonzini, C., 1882, Un Catalogo dei Protozoi da lui raccolti nel Modenese, con un'appendice sui Flagellati: Atti della Società dei Naturalisti di Modena, v. 1, p. 1923.

Bergonzini, C., 1883a, Catalogo dei Protozoi raccolti nel Modenese e suoi dintorni: Atti della Società dei Naturalisti di Modena, Memorie, v. 2, ser. 3, p. 71-80.

Bergonzini, C., 1883b, Osservazione sulla vita e lo sviluppo di alcuni Protozoi: Atti della Società dei Naturalisti di Modena, v. 2, p. 1-18.

Bernard, F., 1947, Protistes pélagiques et couches marines au large d'Alger: Cr. Soc. Biol. Fr. [NOTE: spell out abbreviation, please], v. 141, p. 400-403.

Beyens, L. (1984) A concise survey of testate amoebae analysis. Bulletin de la Société Belge de Géologie, 93 261-266.

Beyens, L. (1985) On the subboreal climate of the Belgian campine as deduced from diatom and testate amoebae analysis. Review of Palaeobotany and Palynology, 46 9-31.

Beyens, L., Chardez, D., 1982, Cryptodifflugia angustastoma new species and Nebela carinatella new species new Testacea from fen bogs in the Belgian Campine: Archiv für Protistenkunde, v. 126, p. 169-172.

Beyens, L., Chardez, D., 1984, Testate amoeba Rhizopoda from Southwest Ireland: Archiv für Protistenkunde, v. 128, p. 109-126.

Beyens, L., Chardez, D. 1987 Evidence from testate amoebae for changes in some local hydrological conditions between c.5000BP and c.3800BP on Edgeøya (Svalbard). Polar Research, 5 165-169.

Beyens, L., Chardez, D. 1995 An annotated list of testate amoebae observed in the Arctic between the longitudes $27^{\circ} \mathrm{E}$ and $168^{\circ} \mathrm{W}$. Archiv für Protistenkunde, 146219 233.

Beyens, L., Chardez, D., de Baere, D., 1991, Ecology of aquatic testate amoebae in coastal lowlands of Devon Island (Canadian High Arctic): Archiv für Protistenkunde, v. 140 , pp. $23-33$

Beyens, L., Chardez, D., de Baere, D., de Bock, P. 1992, The testate amoebae from the Soendre Stroemfjord region (West-Greenland): Their biogeographic implications: 
Archiv für Protistenkunde, v. 142, pp. 5-13.

Beyens, L., Chardez, D., de Baere, D., de Bock, P., Jacques, E., 1988, Some data on the testate amoebae from the Shetland Islands and the Faeroeer: Archiv für Protistenkunde, v. 136, pp. 79-96.

Beyens, L., Chardez, D., de Baere, D., Verbruggen, C., 1995, The aquatic testate amoebae fauna of the Stroemness Bay area, South Georgia: ANTARCT.-SCI., v. 7, pp. 3-8. [SPELL OUT ABBREVIATION]

Beyens, L., Chardez, D., and de Landtsheer, R. 1986 Testate amoebae from moss and lichen habitats in the Arctic. Polar Biology, 5 165-173.

Beyens, L., Chardez, D., de Landtsheer, R., de Baere, D., 1986, Testate amoebae communities from aquatic habitats in the Arctic: Polar Biology. v. 6, pp. 197-205. 


\section{BHA-BOV}

Bhatia, B., 1930, On some freshwater Rhizopods and flagellates from Kashmir: Archiv für Protistenkunde, v. 72, p. 156-167.

Biczok, F., 1954, Testaceen in der Rhizosphare: Annales Universitatis Scientiarum Budapestiensis de Rolando Eötvös Nominatae (Sectio biologica), v. 2, p. 385-394.

Biernacka, l., 1956, Przyczynek do znajomoci pierwotniakw Zalewu Wilanego: Polskie Archiwum Hydrobiologii, v. 3, no 16, p. 43-68.

Biernacka, I., 1959, Plankton Jeziora Roznowskiego w czerwcu 1957: Polskie archiwum hydrobiologii, tom. XVIII, NR. 2, p. 147-159.

Blainville, H. M. D. de, 1834-1837, Manuel d'Actinologie ou de Zoophytologie: 2 volumes, Volume I, text: Volume II, atlas of plates (pages unknown): F.- G. Levrault (Paris).

Blainville, H. M. D. de, Milne-Edwards, H., 1839, Rapport sur un Mémorie de M. P. Gervais, intitulé: Observations pour servir à l'histoire des Polypes d'eau douce: Compte rendu des Séances de l'Académie des Sciences, v. 8, no 14, p. 522-527.

Blanc, H., 1884, Rhizopods nouveaux pour la faune profonde du Lac Leman: Belletin de la Société Vaudoise des Sciences Naturelles, v. 20, p. 287-288.

Blanc, H., 1885, New Rhizopoda of the deep-water fauna of the Lake of Geneva: Annales and Magazine of Natural History, v. 15, p. 427.

Blanc, H., 1886, Un nouveau Foraminifère de la faune profonde du Lac [Léman]: Archives des Science physiques et naturelles (Genève), sèr. 3, v. 16, p. 362-366.

Blanc, H., 1891, Sur quelques Protistes dragués au fond du lac Léman appartenant au genre Difflugia: Archives des Science physiques et naturelles (Genève), sèr. 3, v. 26, p. 576-577.

Blanc, H., 1892, Les difflugies de la faune profonde du Lac Léman, in: Recueil Inaugural de l'Université de Lausanne 1892, p. 377-387, pl. 2.

Bles, E. J., 1929, Arcella. A study in cell physiology: Quarterly Journal of Microscopic Science, new ser., v. 72, no 288, p. 527-648, pls. 30-40.

Blochmann, F., 1886, Die mikroskopische Thierwelt des Süsswasser, Braunschweig - no further information, probably a book.

Blochmann, F., 1887, Zur Kenntniss der Fortpflanzung von Euglypha alveolata Dujardin: Morphologische Jahrbuch, v. 13, no 1, p. 173-183, pl. 5.

Blochmann, F., 1888, Contributions to the knowledge of the reproduction of Euglypha 
alveolata Dujardin: Annals and Magazine of Natural History, ser. 6, v. 1, p. 27-36, pl.4. - Translation of Blochmann 1887.

Blochmann, F., 1894, Über die Kerntedung bei Euglypha: Biologisches Zentralblatt, v. 14, p. $194-197$.

Blochmann, F., 1895, Protozoa: p. 1-134, pls. 1-8, in: Blochmann, F. and Kirchener, ?., 1895, Die mikroskopische Tier- und Pflazenwelt des Süsswasser, Lucas Gräfe and Sillem (Hamburg).

Bobrov, A.A., Yazvenko, S.B., Warner, B.G., 1995, Taxonomic and ecological implications of shell morphology of three testaceans (Protozoa: Rhizopoda) in Russia and Canada: Archiv für Protistenkunde, v. 145, pp. 119-126

Bolli, H. M., Saunders, J. B., 1954, Discussion of some Thecamoebina described erroneously as Foraminifera: Cushmann Foundation for Foraminiferal Research, Contributions, v. 5, no 2, p. 45-52.

Bolton, T., 1886, Microorganisms in a swampy ditch in Sutton Park: Midland Naturalist, v. 9, p. 173-176.

Boltovskoy, E., 1956, Contribucin al conocimiento de las tecamebas del Ro de - la Plata: Acta Geologica Lilloana (Tucuman), v. I, p. 298-313, 1 pl.

Boltovskoy, E., Boltovskoy, A., 1968, Foraminferos y Tecamebas de la parte inferior del Ro Quequén Grande, Provincia de Buenos Aires, Argentina (sistemtica, distribucin, ecologa): Revista del Museo Argentino de Ciencias Naturales "Bernardino Rivadavia" e Instituto Nacional de Investigacin de las Ciencias Naturales, Hidrobiologia, v. 2, no 4. p. 127-166, pls. 1-3.

Boltovskoy, E., Lena, H., 1966, Contribucin al conocimiento de las tecamebas de Ushuaia (Tierra del Fuego, Argentina): Neotropica, v. 12, p. 55-65, 1 pl.

Boltovskoy, E., Lena, H., 1971a, Contribution a l'etude des thecamoebiens de la Province de Buenos Aires: Hydrobiologia, v. 38, pp. 441-451.

Boltovskoy, E., Lena, H., 1971b, Tecamebas de Isla de los Estados (Argentina): Revista Española de Micropaleontologia, v. 3, pp. 129-140.

Boltovskoy, E., Lena, H., 1971a, Contribution à l'étude des Thécamoebiens de la Province de Buenos Aires: Hydrobiologia, v. 38, no 3-4, p. 441-450, pls. 1-3.

Boltovskoy, E., Lena, H., 1971b, Tecamebas de Isla de los Estados: Revista Española de Mícropaleontologia, v. 3, no 2, p. 129-140.

Boltovskoy, E., Lena, H., 1974, Tecamebas del Río de la Plata: Servício de Hydrografia Naval, Publicación H660, p. 1-32, 6 pls.

Bonnet, L., 1953, Thécamoebiens de quelques stations du midi de la France et sur les 
Thécamoebiens de la region d'Oredon (Hautes Pyrénées): Bulletin de la Société d'Histoire Naturelle de Toulouse, v.88, p. 1-2.

Bonnet, L., 1958, Les Thécamoebiens du sol: Bulletin de la Société d'Histoire Naturelle de Toulouse, v.93, p. 529-543.

Bonnet, L., 1959a, Dékystement, phase trophique et enkystement chez Plagiopyxis minuta Bonnet (Thécamoebiens). Incidences systématiques: Comptes rendus hebdomadaires des Séances de l'Académie des Sciences, Paris, v. 249, no 23, p. 2617-2619.

Bonnet, L., 1959b, Nuveaux Thécamoebiens du sol: Bulletin de la Société d'Histoire Naturelle de Toulouse, v. 94, no 1-2, p. 177-188, pls. 1-2.

Bonnet, L., 1959c, Quelques aspects des populations Thécamoebiennes endogées: Bulletin de la Société d'Histoire Naturelle de Toulouse, v. 94, p. 413-428.

Bonnet, L., 1960a, Thécamoebiens du sol d'Angola: Publicaçes culturais da Companhia de Diamantes de Angola, no ?, p. 78-86.

Bonnet, L., 1960b, Nouveaux Thécamoebiens du sol (III): Bulletin de la Société d'Histoire Naturelle de Toulouse, v. 95, no 3-4, p. 209-211.

Bonnet, L., 1960c, Kystes et Trophophase chez Plagiopyxis minuta Bonnet (Thécamoebiens): Bulletin de la Société Zoologique de France, v. 85, p. 43-52.

Bonnet, L., 1961a, L'émission pseudopodique chez les thécamoebiens endogés (I): Bulletin de la Société Zoologique de France, v. 86, no 1, p. 17-28.

Bonnet, L., 1961b, Caractéres generaux des populations thécamoebiennes endogées: Pedobiologia, v. 1, p. 6-24.

Bonnet, L., 1962, Biologie de l'Amérique Australe: Centre National de la Recherche Scientifique, v. 1, p. 43-47.

Bonnet, L., 1964, Le peuplement thécamoebien des sols: Revue d'Écologie et de Biologie du Sol, v. 1, no 2, p. 123-408, pls. 21-22.

Bonnet, L., 1965a, Thécamoebiens d'Orédon: Annales de Limnologie, v. 1, no 2, p. 113-143.

Bonnet, L., 1965b, Sur le peuplement thécamoebiens de quelques sols du Spitzbergen: Bulletin de la Société d'Histoire Naturelle de Toulouse, v. 100, no 3-4, p. 281-293.

Bonnet, L., 1965c, Nouveaux thécamoebiens du sol: Bulletin de la Société d'Histoire Naturelle de Toulouse, v. 100, no 3-4, p. 330-332.

Bonnet, L., 1966a, Le peuplement thécamoebien de quelques sols du Chili: 
Protistologica, v. 2, no 2, p. 113-140, pls. 1-2.

Bonnet, L., 1966b, Le peuplement thécamoebiens des sols du Gabon: Biologica Gabonica, v. 2, no 3, p. 183-214.

Bonnet, L., 1967a, Le peuplemet thécamoebiens du sol de Gréce: Biologica Gallohellenica, v. 1, no 1, p. 7-26.

Bonnet, L., 1967b, Le peuplemet thécamoebiens de quelques sols de la Republique du Congo-Brazza: O.R.S.T.O.M. Série Biologique, no 3, p.43-53.

Bonnet, L., 1969a, Thécamoebiens des sols de l'Angole (II): in: Subsídios para o estudo de biologia na Lunda: p. 115-135, pl. 5, Publicaçes culturais da Companhia de Diamantes de Angola, no 81.

Bonnet, L., 1969b, Aspects généraux du peuplement thécamoebiens édaphique de l'Afrique intertropicale: in: Subsídios para o estudo de bìologia na Lunda: p. 137-176, pl. 5, Publicaçes culturais da Companhia de Diamantes de Angola, no 81.

Bonnet, L., 1970a, Les Distomatopyxidae fam. nov. et la structure diplostome chez les Thécamoebiens (Rhizopoda Testacea): Comptes rendus des Séances de l'Académie des Sciences, Paris, v. 271, sér. D, p. 1189-1191.

Bonnet, L., 1970b, Le peuplemet thécamoebiens du sol de Gréce (suite).Modéles mathématiques du nilieu et de la popolation: Biologica Gallo-hellenica, v. 3, no 1, p. 521.

Bonnet, L., 1970c, Nouveaux Thécamoebiens du sol (VI): Bulletin de la Société d'Histoire Naturelle de Toulouse, v. 106, fasc. 3-4, p. 328-332, pl. 1.

Bonnet, L., 1972a, Aspects généraux du peuplement thécamoebiens des sols de l'Auvergne: Annales de la Station Biologique de Basse-en-Chandesse, no 6-7, p. 12.

Bonnet, L., 1972b, Premiere relevé de certaines groupes de protozoaires libres sur le territoire de la station de Biologie de l'Université de Montréal: Naturaliste Canadien, v. 99, p. 417-440.

Bonnet, L., 1973a, Aspects généraux du peuplement des mousses coticoles: in: De Puytorac, P., Grain, J. (eds.): Progress in Protozoology. Abstracts of papers read at the Fourth International Congress on Protozoology, Clermont-Ferrand, Sept. 1973: p. 51 (Clermont-Ferrand).

Bonnet, L., 1973b, A propos de Geopyxella sylvicola et de Pseudawerintzewia calcicola (Rhizopods Thécamoebiens édaphiques): Revue d'Ecologie et de Biologie du Sol, v. 10, no 4, p. 509-522.

Bonnet, L., 1973c, Le peuplement thécamoebiens des mousses corticoles: 
Protistologica, v. 9, p. 319-338.

Bonnet, L., 1974a, Ecologie des Protistes du sol: in: De Puytorac, P., Grain, J. (eds.): Actualités Protozoologiques, v. I. Résumé des discussions et des Tables Rondes du 4e Congrès International de Protozoologie, Clermont-Ferrand, Sept. 1973: p. 239-250, (Clermont-Ferrand).

Bonnet, L., 1974b, Observations préliminaires sur le peuplement thécamoebiens des sols de Lamto et du Massif du Nimba: Bulletin de liaison des Chercheurs du Lamto, P.B.I., p. 25-28.

Bonnet, L., 1974c, Les Lamtopyxidae fam.nov. et la structure propylostome chez les Thécamoebiens (Rhizopoda Testacea): Comptes rendus des Séances de l'Académie des Sciences, Paris, v. 278, p. 2935-2937.

Bonnet, L., 1974d, Quelques aspects dun peuplement thécamoebiens des sols de la province de Québec (Canada): Canadian Journal of Zoology, v. 52, no 1, p. 29-91, pl. 1.

Bonnet, L., 1974e, Quelques aspects du peuplement thécamoebiens des sols des truffières: Protistologica, v. 10, fasc. 3, p. 281-291.

Bonnet, L., 1974f, Nouveau Thécamoebiens du sol (VII): Bulletin de la Société d'Histoire Naturelle de Toulouse, v. 110, fasc. 3-4, p. 283-290.

Bonnet, L., 1974g, Lamtoquadrula gen. nov, et la structure plagiuostome chez les Thécamoebiens nébéliformes: Bulletin de la Société d'Histoire Naturelle de Toulouse, v. 110, fasc. 3-4, p. 297-299.

Bonnet, L., 1975a, Nouvelles données sur le peuplement thécamoebien des sols de Lamto et du Nimba: Bulletin de liaison des chercheurs de Lamto, P.B.I., p. 21-24.

Bonnet, L., 1975b, Nouveaux Thécamoebiens du sol (VIII): Bulletin de la Société d'Histoire Naturelle de Toulouse, v. 111, no 3-4, p. 300-302.

Bonnet, L., 1975c, Type morphologique, écologie et évolution de la thèque chez les Thécamoebiens: Protistologica, v. 11, no 3, p. 363-378.

Bonnet, L., 1976a, Caractéres généraux du peuplement thécamoebien des sols calcimorphes: Journal of Protistology, v. 23, no 4, p. 5a.

Bonnet, L., 1976b, Le peuplement thécamoebien édaphique de la Côte-d'Ivoire. Sols de la région de Lamto: Protistologica, v. 12, no 4, p. 539-554.

Bonnet, L., 1977a, Thécamoebiens et potentialités truffières des sols: Abstracts: 16138me Réunion du Groupement des Protistologues de Langue Française, p. 21 - Stencilled document. [NOTE: is number after ABSTRACTS correct?]

Bonnet, L., 1977b, Faunistique et biogéographie des Thécamoebiens. I. 
Thécamoebiens des sols du Mexique: Bulletin de la Société d'Histoire Naturelle de Toulouse, v. 113, no 1-2, p. 40-44.

Bonnet, L., 1977c, Faunistique et biogéographie des Thécamoebiens. II. Thécamoebiens des sols de la Guadeloupe et de la Martinique: Bulletin de la Société d'Histoire Naturelle de Toulouse, v. 113, no 1-2, p.45-49.

Bonnet, L., 1977d, Nouveaux Thé du sol (IX): Bulletin de la Société d'Histoire Naturelle de Toulouse, v.113, no 1-2, p. 152-156.

Bonnet, L., 1977e, Le peuplement thécamoebien des sols du Népal et son intêrêt biogéografique: Bulletin de la Société d'Histoire Naturelle de Toulouse, v. 113, no 34, p. 331-348.

Bonnet, L., 1978a, Faunistique et biogéographie des thécamoebiens. IV. Thécamoebiens des sols du massif du Nimba (Côte-d'voire): Bulletin de la Société d'Histoire Naturelle de Toulouse, v. 114, no 1-2, p. 59-64.

Bonnet, L., 1978b, Le peuplement thécamoebiens des sols calcimagnésiques: Revue d'Ecologie et de Biologie du sol, v. 15, no 2, p. 169-190.

Bonnet, L., 1978c, Faunistique et biogéographie des Thécamoebiens. III. Thécamoebiens des épiphytes de la région de Lamto (Côte-d'Ivoire): Bulletin de la Société d'Histoire Naturelle de Toulouse, v. 114, no 1-2, p. 51-58.

Bonnet, L., 1979a, Origine et biogeographie des Distomatopyxidae (Rhizopodes Thécamoebiens des sols, Lobosia, Arcellinida): Comptes rendus des Séances de l'Académie des Sciences, Paris, sér. D, v. 288, no 8, p. 775-778.

Bonnet, L., 1979b, Nouveaux Thécamoebiens du sol (X): Bulletin de la Société d'Histoire Naturelle de Toulouse, v. 115, no 1-2, p. 106-118.

Bonnet, L., 1979c, Faunistique et biogéographie des Thécamoebiens du Brésil et du Paraguay: Bulletin de la Société d'Histoire Naturelle de Toulouse, v. 115, no 1-2, p. 119-122

Bonnet, L., 1979d, Les Thécamoebiens (Rhizopoda Testacea) des sols: p. 157-172, in: Sauvage et al.: Les biocénoses halophiles de Camargue, La Terre et la Vie, Revue d'Ecologie appliquée, supplément 2, Numèro special 2.

Bonnet, L., 1980a, Nouveaux Thécamoebiens du sol: Bulletin de la Société d'Histoire Naturelle de Toulouse, v. 116, n167 3-4, p. 255-265.

Bonnet, L., 1980b, Quelques aspects de la faune thécamoebiennes des sols de la Nouvelle-Guinée (Papouasie): Bulletin de la Société d'Histoire Naturelle de Toulouse, v. 116, no 3-4, p. 266-276.

Bonnet, L., 1980c, Faunistique et biogéographie des Thécamoebiens. VI. 
Thécamoebiens de quelques sols des Philippines: Bulletin de la Société d'Histoire Naturelle de Toulouse, v. 116, n167 3-4, p. 277-282.

Bonnet, L., 1981a, Faunistics and bio geography of the thecamoebins 6:

Thecamoebians of some soils from the Philippines: Bulletin de la Société d'Histoire Naturelle de Toulouse, v. 116, p. 277-282.

Bonnet, L., 1981b, L'intêrêt biogéographique du peuplement Thécamoebien des sols du Népal: Comptes rendu, Paleogéographie et Biogéographie de l'Himalaya et du Sous-Continant Indien, Table Ronde, Paul Sabatier Universitè de Paris, p. 53-60.

Bonnet, L., 1981c, Some aspects of the thecamoebian fauna of the soils of New Guinea Papua: Thecamoebians of some soils from the Philippines: Bulletin de la Société d'Histoire Naturelle de Toulouse, v. 116, p. 266-276.

Bonnet, L., 1984a, Ecologic citation of the thecamoebians of the soil: Bulletin de la Société d'Histoire Naturelle de Toulouse, v. 120, p. 103-110.

Bonnet, L., 1984b, New data on the genus Planhoogenraadia Thecamoebians: Bulletin de la Société d'Histoire Naturelle de Toulouse, v. 120, p. 117-122.

Bonnet, L., 1984c, Plagiopyxis with a Plagiopyxis callida structure soil Thecamoebians: Protistologica, v. 20, p. 475-490.

Bonnet, L., 1988d, Ecological description of soil thecamoebians 3: Characteristics of the facies: Bulletin de la Société d'Histoire Naturelle de Toulouse, v. 116, p. 7-12.

Bonnet, L., 1988e, The ecology of the genus Plagiopyxis soil thecamoebians: Bulletin de la Société d'Histoire Naturelle de Toulouse, v. 124, p. 13-22.

Bonnet, L., Brabet, J., Comoy, N., Guitard, J., 1978, Ultrastructure et composition de la thèque chez quelques Thécamoebiens: Journal of Protozoology, v. 25, no 3, part 1, p. $48 \mathrm{a}-49 a$.

Bonnet, L., Brabet, J., Comoy, N., Guitard, J., 1979, Nouvelles observations sur l'ultrastructure de la paroi de la thèque chez les Thécamoebiens (Rhizopodes

Testacés): Comptes Rendus de l'Académie des Sciences, Paris, sér. D, v. 288, no 2, p. 227-230.

Bonnet, L., Brabet, J., Comoy, N., Guitard, J., 1981a, New data on the thecamoebian Filosia Amphitrema flavum: Protistologica, v. 17, p. 225-234.

Bonnet, L., Brabet, J., Comoy, N., Guitard, J., 1981a, Nouvelle données sur le thécamoebien filosia Amphitrema flavum (Archer 1877) Penard 1902: Protistologica, v. 17, no 2, p.225-233, pl.17.

Bonnet, L., Brabet, J., Comoy, N., Guitard, J., 1981b, Observations sur l'ultrastructure de Nebela marginata (Rhizopoda, Testacea, Lobosia, Hyalosphenidae): Protistologica, 
v. 17, p. 235-341, pl. 17.

Bonnet, L., Comoy, N., 1977, Les groupements thécamoebiens de quelques sols du littoral français méditerranéen: Bulletin de la Société d'Histoire Naturelle de Toulouse, v. 113, no 3-4, p. 349-365.

Bonnet, L., Gomez-Sanchex, M.- S., 1984: Preliminary note on the thecamoebian population of soils of Asturias Spain: Bulletin de la Société d'Histoire Naturelle de Toulouse, v. 120, p. 111-116.

Bonnet, L., Thomas, R., 1955, Étude sur les Thécamoebiens du sol (I): Bulletin de la Société d'Histoire Naturelle de Toulouse, v. 90, n167 3-4, p. 411-428.

Bonnet, L., Thomas, R., 1958, Une téchnique d' isolement des thécamoebiens (Rhizopoda Testacea) du sol et ses résultats:Comptes rendus hebdomadaires des Séances de l'Académie des Sciences, Paris, v. 247, p. 1901-1903.

Bonnet, L., Thomas, R., 1959, Nouveaux Thécamoebiens du sol (II): Bulletin de la Société d'Histoire Naturelle de Toulouse, v. 94, p. 407-412.

Bonnet, L., Thomas, R., 1960a, Étude sur les Thécamoebiens du sol: Bulletin de la Société d'Histoire Naturelle de Toulouse, v. 95, no 3-4, p. 339-349.

Bonnet, L., Thomas, R., 1960b, Thécamoebiens du sol: in: Hermann (ed.), Faune terrestre et d'eau douce des Pyrénées-Orientales, no 5, 113 p., Supplement to Vie et Milieu, (Paris).

Bovee, E. C., ??year?? Distribution and forms of siliceous structures among Protozoa: in: Simpsom T. C., Volcani, B. E. (eds.): Chapter 9, Silicon and siliceous structures in biological systems.?? p. 1-587, Springer (New York). [NOTE MISSING INFORMATION]

Bovee, E. C., 1960, Protozoa of the Mountain Lake region, Giles County, Virginia: Journal of Protozoology, v. 7, no 4, p. 353-361.

Bovee, E. C., 1977, Sarcodina: in: Hurlbert, S. H. (ed.): Biota acuatica de Sudamerica Austral, p. 48-54, San Diego State University Press, San Diego (California).

Bovee, E. C., Jahn, T. L., 1960, Locomotion and the classification of Amoebida and Testacida: Journal of Protozoology, v. 7 (suppliment), p. 8.

Bovee, E., C., 1979, Protozoa from acid-bog mosses and forest mosses of the Lake Itasca region (Minnesota, U.S.A.): University of Kansas Science Bulletin, v. 51, no 21 , p. $615-629$. 


\section{BRA-CAV}

Brabet, J., Comoy, N., Guitard, J., Bonnet, L., 1983, Some data on the ultrastructure of Heleopera petricola amethystea Rhizopoda testacea: Bulletin de la Société d'Histoire Naturelle de Toulouse, v. 118, p. 161-168.

Bradley, W. H., 1931, Origin and microfossils of the oil shale of the Green River Formation of Colorado and Utah: U. S. Geological Survey, Professional Paper 168, p. I-VI, 1-58.

Brandwein, P., 1937, Culture of some freshwater Rhizopoda: in: Galtsoff, P. S., Lutz, F. E., Welch, P. S., Needham, J. G. (eds.): Culture methods for invertebrate animals, $p$. 72-74, Comstock Publishing Company Inc., Ithaca (New York).

Brightwell, T., 1848, Sketch of a fauna Infusoria, for East Norfolk: Printed by J. Fletcher, p. I-VIII, 1-40, (Norwich).

Bronn, H. G., 1859, Die Klassen und Ordnungen der formlosen Thiere (Amorphozoa) wissenschaftlich dargestellt in Wort und Bild: in: Bronn, H. G.: Die Klassen und Ordnungen des Thier-Reichs, wissenschaftlich dargestellt in Wort und Bild. I. Band: Amorphozoen, p. I-XVI, 1-142, pls. 1-12, C. F. Winter, Leipzig and Heidelberg.

Brown, J. M., 1909, Freshwater Rhizopods from the Sheffield district: Naturalist, a monthly journal of natural history for the North of England, p. 105-108.

Brown, J. M., 1910a, Rhizopods from the Sheffield district: Naturalist, a monthly journal of natural history for the North of England, p. 91-93.

Brown, J. M., 1910b, Freshwater Rhizopods from the English Lake District: Journal of the Linnean Society of London, Zoology, v. 30, no 201, p. 360-368, pl. 50.

Brown, J. M., 1910c, Protozoa: in: Handbook and guide to the Sheffield district. p. 499-502, publisher (Sheffield).

Brown, J. M., 1911a, A contribution to our knowledge of the freshwater Rhizopoda and Heliozoa of Scotland: Annals of Scottish Natural History, v. 20, no 80, p. 226-232.

Brown, J. M., 1911b, Observations on some new and little-known British Rhizopods: Journal of the Linnean Society of London, Zoology, v. 32, no 212, p. 77-85, pl.9.

Brown, J. M., 1912a, Further contributions to our knowledge of the Rhizopoda and Heliozoa of Scotland: Scottish Naturalist, p. 108-114.

Brown, J. M., 1912b, Freshwater Rhizopoda and Heliozoa from Kinder Scout: Naturalist, no 665, p. 179-182.

Brown, J. M., 1913a, A further contribution to our knowledge of the Rhizopods of Scotland: Scottish Naturalist, p. 185-188, 205-210. 
Brown, J. M., 1915, On the occurrence of Paulinella chromatophora in Britain: Naturalist, p. 157-159.

Brown, J. M., 1918, On Pyxidicula invisitata, a rhizopod new to Britain, and Hedriocystis spinifera, a new Heliozoon: Journal of the Royal Microscopical Society, no 2, p. 170-172.

Buck, E., 1877, Einige Rhizopodenstudien: Zeitschrift für wissenschaftliche Zoologie, v. 30, no 1, p. 1-49, pls. 1, 2.

Buisan, H. G., 1946, Contribución al estudies a los Protozos de las aguas dulces: Instituto de Biologia Aplicada. , Publicaciones (Barcelona), ?? pages.

Bunescu, V. I., 1971a, Presenta testacelor in unele soluri din Massivul Valdeasa: Stiinta solului, v. 9, no 3, p. 45-5?.

Bunescu, V. I., 1971b, Contribuii la studuil testaceelor din soluril Muntilor Bucegi: Institutal Agronomica Timisoara Lucrraris Tiintifice. Seria Zootehnie, v. 27, p. 3138.

Bunescu, V. I., 1974, Testaceele (Protozoa: Rhizopoda Testacea) din solurile Muntilor Bucegi: Rezumat teza de doctorat, Universitatea "Babes-Bolyai", Cluj-Napoca: ??pages.

Bunescu, V. I., 1977, Testaceele (Protozoa: Rhizopoda Testacea) din solurile litomorfe (Muntii Bucegi-Romania): Publicatiile S. N. Republicii Socialiste Romania, v. 16, p. 267-275.

Bunescu, V.I., 1981, The Testacea of the Podzolic brown soils in the Bucegi Mountains Romania: Buletinul Institutului Agronomic Cluj-Napoca Seria Agricultura, v. 35, p. 15-28.

Bunescu, V. I., Matic, Z., Blaga, G., Moldovan, I., 1985, Testacea Protozoa Rhizopoda in forset litters of the Bucegi Mountains Romania: Studia Universitatis Babes-Bolyai Biologia, v. 30, p. 25-29.

Burbidge, S.M. 1997 Holocene Environmental History of Lake Winnipeg:

Thecamoebians and stable lead isotopes. Ph.D. Dissertation, Carleton University, Ottawa, Ontario Canada 186pp.

Burbidge, S.M, Patterson, R.T., Schroeder-Adams, C.J., 1994, Holocene thecamoebians (arcellaceans) as environmental indicators for lakes in southern New Brunswick, Canada: Abstract in: Geological Society of America, 1994 annual meeting, Boulder, CO, United States.

Burbidge, S. M. and Schroder-Adams, 1998. Thecamoebians in Lake Winnipeg: a tool for Holocene paleolimnology, Journal of Paleolimnology 19: 309-328. 
Burgess, A., 1958, Micro-organisms in the soil: in: Biological Sciences, p. 1-188, Hutchinson University Library (London).

Butler, J. B., 1908, Protozoa: in: Handbook to the City of Dublin and surrounding districts, p. 216-220, ??publisher, (Dublin).

Butschinsky, P., 1897, Die Protozoen-Fauna der Salzsee-Limane bei Odessa: Zoologischer Anzeiger, v. 20, no 533, p. 194-197.

Bütschli, O., 1873, Einiges über Infusorien: Archiv für Mikrosopische Anatomie, v. 9, p. 657-678.

Bütschli, O., 1875, Zur Kenntniss der Fortpflanzung bei Arcella vulgaris Ehr.: Archiv für mikroskopische Anatomie, v. 11, p. 459-467, pl. 25.

Bütschli, O. (1880) Erster Band. Protozoa. In: Bronn, H.G. Klassen und Ordnungen des Their-Reichs. I Band, I Abth, 1-224.

Bütschli, O. 1880-1889, Protozoa. Mit einem Beitrag: Palaeontologische Entwicklung der Rhizopoda, von C. Schwager, in: Bronn, H. G. (ed.), Die Klassen und Ordnungen des Thierreichs, wissenschaftlich dargestellt in Wort und Bild: Winter'sche Verlagshandlung (Leipzig), 3 volumes: Volume 1. 1880-1888, Abtheilung: Sarkodina und Sporozoa, p. I-XVIII, 1-616, pls. 1-38; Volume 2. 1883-1885, Abtheilung:

Mastigophora, p. 617-1097, pls. 39-55; Volume 3. 1887-1889, Abtheilung: Infusoria und System der Radiolaria, p. I-VII, 1098-2035, pls. 56-79.

Buttler, A., Warner, B.G., Grosvernier, P. \& Matthey, Y. (1996) Vertical patterns of testate amoebae (Protozoa: Rhizopoda) and peat-forming vegetation on cutover bogs in the Jura, Switzerland. New Phytologist, 134 371-382

Cambar, R., Leblanc, M., Mercier, M., Thomas, R., 1964, Contribution à l'étude d'ultrastructures superficcelles de protistes: constitution de la thèque de quelques Rhizopodes testacés: in: Titlbach, M. (ed.), Electron Microscopy 1964- Proceedings of the Third European Regional Conference held in Prague, August 26- September 3, 1964, v. B, p. 185-186: Publishing House of the Czechoslovak Academy of Sciences (Prague).

Cambar, R., Thomas, R., Leblanc, M., 1963, Recherches sur la constitution de la theques des Arcelles (Genre Arcella, Rhizopoda testacea) observations au microscope electronique: Compte Rendu Hebdomadaire des Seances de l'Académie des Sciences, ser. D, v. 256, p. 1364-1366.

Cantor, T., 1842, General features of Chusan, with remarks on the flora and fauna of that island: Annals and Magazine of Natural History, ser. 1, v. 9, no 60, p. 481-493.

Carpenter, W. A., 1891a, The microscope and its revelations, 7th edition, prepared with the assistance of W. H. Dallinger: p. I-XVIII, 1-1099, 20 plates: J. and A. Churchill (London). 
Carpenter, W. A., 1891b, The microscope and its revelations, 7th edition, prepared with the assistance of W. H. Dallinger: p. I-XVIII, 1-1099, 20 plates: P. Blakiston, Son, and Co. (Philadelphia).

Carpenter, W. B., 1856a, The microscope and its revelations, 1st edition: $p$. V-XX + 1-778: J. Curchill (London).

Carpenter, W. B., 1856b, The microscope and its revelations, p. I-XXIV, 33-724: Blanchard and Lea (Philadelphia).

Carpenter, W. B., 1857, The microscope and its revelations, 2nd edition: p. I--XIX, 1-719: J. Churchill (London).

Carpenter, W. B., 1861, On the systematic arrangement of the Rhizopoda: Natural History Review (Dublin and London), new series, v. 1, no 4, p. 456-472.

Carpenter, W. B., 1862, The microscope and its revelations, 3rd edition: p. 1--792: J. Churchill (London).

Carpenter, W. B., 1868, The microscope and its revelations, 4th edition: p. I-XXVIII, 1-796, pls. 1-25: J. Churchill and Sons (London).

Carpenter, W. B., 1875, The microscope and its revelations, 5th edition, prepared with the assistance of H. J. Slack: p. I-XXXII, 1-848, pls. 1-25: J. and A. Churchill (London).

Carpenter, W. B., 1881a, The microscope and its revelations, 6th edition: p. 1-882, pls. 1-26: J. and A. Churchill (London).

Carpenter, W. B., 1881b, The microscope and its revelations, 6th edition: 2 volumes, pages unknown, 26 plates: Wood (New York).

Carpenter, W. B., 1881c, The microscope and its revelations, 6th edition: p. IXXXII, 1-882, pls. 1-25: P. Blakiston (Philadelphia).

Carpenter, W. B., 1883, The microscope and its revelations, 6th edition: 2 volumes, pages unknown: "Wood's Library of standard medical authors", Wood (New York).

Carpenter, W. B., 1901a, The microscope and its revelations, 8th edition, prepared with the assistance of W. H. Dallinger: p. I-XX, 1-1181, pls. 1-22: J. and A. Churchill (London).

Carpenter, W. B., 1901b, The microscope and its revelations, 8th edition, prepared with the assistance of W. H. Dallinger: p. I-XX, 1-1181, pls. 1-22: Blakiston (Philadelphia).

Carter, F. B., 1888, Rhizopoda, their life-history and classification: American Monthly Microscopical Journal, v. 9, p. 3-10. 
Carter, H. D., 1870, On two new species of the foraminiferous genus Squamulina; and on a new species of Difflugia: Annals and Magazine of Natural History, ser. 4, v. 5, no 29, p. 309-326, pl. 4, 5.

Carter, H. J. 1857b, On the ultimate structure of Spongilla, and additional notes on freshwater Infusoria: Annals and Magazine of Natural History, ser. 2, v. 20, no 115, p. 21-41, pl. 1.

Carter, H. J., 1856a, Notes on the freshwater Infusoria of the island of Bombay: Annals and Magazine of Natural History, ser. 2, v. 18, no 104, p: 115-132.

Carter, H. J., 1856b, Notes on the freshwater Infusoria of the island of Bombay: Annals and Magazine of Natural History, ser. 2, v. 18, no 105, p. 221-249, pls. 5-7.

Carter, H. J., 1857, Additional notes on the freshwater Infusoria of the Island of Bombay: Annals and Magazine of Natural History, v. 20, p. 34-41.

Carter, H. J., 1863, On the presence of chlorophyll-cells and starch-granules as normal parts of the organism, and on the reproductive process, in Difflugia pyriformis, Perty; also on a freshwater species of Echinocystidia: Annals and Magazine of Natural History, ser. 3, v. 12, no 70, p. 249-264.

Carter, H. J., 1864, On freshwater Rhizopoda of England and India: Annals and Magazine of Natural History, ser. 3, v. 13, no 73, p. 18-39, pls. 1-2.

Carter, H. J., 1865, On the fresh- and salt-water Rhizopoda of England and India: Annals and Magazine of Natural History, ser. 3, v. 15(?), no 88, p. 277-293, pl. 12.

Cash, J., 1892, The fresh-water Rhizopoda of the Manchester district: Transactions of the Manchester Microscopical Society, p. 47-55, pl. 2.

Cash, J., 1904, On some new and little known British freshwater Rhizopoda: Journal of the Linnean Society of London, Zoology, v. 29, p. 218-225, pl. 26.

Cash, J. and Hopkinson, J., 1905, The British freshwater Rhizopoda and Heliozoa: v. I: Rhizopoda, Part I: Ray Society (London) publication no 85, p. i-ix, 1-151, pls. 1-16.

Cash, J. and Hopkinson, J., 1909, The British freshwater Rhizopoda and Heliozoa: v. II: Rhizopoda, Part II: Ray Society (London) publication no 89, p. i-xviii, 1-166, pls. 17-32.

Cash, J., Wailes, G.H. and Hopkinson,J. (1915) The British Freshwater Rhizopoda and Helioza: Vol 3 Rhizopoda part 3. The Ray Society, London, pp 156.

Cash, J., Wailes, G. H. and Hopkinson, J., 1919, The British freshwater Rhizopoda and Heliozoa: Ray Society (London) publication no 103, v. IV, p. i-xii, 1-130, pls. 5863. The volume contains: (1) Wailes, G. H., Supplement to the Rhizopoda: p. 1-71, pls. 58-63; (2) Hopkinson, J., Bibliography of v. I-IV, p. 72-122, General index of the 
Rhizopoda: p. 123-130.

Casper, S. J., Schönborn, W., 1985, Difflugia limnetica (Levander) Penard (Protozoa: Testacea) as indicator organism of calcite precipitation in Lake Stechlin, GDR: Archiv für Protistenkunde, v. 130, p. 305-311.

Cattaneo, G., 1877, Intorno all' ontogenesi dell' Arcella vulgaris Ehr.: Atti della Società italiana di Scienze naturali, v. 20, p. 331-343, pl. 6.

Cattaneo, G., 1879a, Intorno ai Rizopodi: Bollettino scientifico (Pavia), v. 1, anno 1, no 1, p. 6-8.

Cattaneo, G., 1879b, Intorno ai Rizopodi: Bollettino scientifico (Pavia), v. 1, anno 1, no 2, p. 25-29.

Cattaneo, G., 1879c, Intorno ai Rizopodi: Bollettino scientifico (Pavia), v. 1, anno 1, no 3, 4, p. 50-61.

Cattaneo, G., 1882, Sui Protisti del Lago di Como: Bollettino scientifico (Pavia), v. 1, anno 3, no 4, p. 111-121.

Cavallini, F., 1926a, ?? title: Journal of Experimental Zoology, v. 43, pages (?)

Cavallini, F., 1926b, ?? title: Journal of Experimental Zoology, v. 43,pages (?) 


\section{CER-CLA}

Certes, A., 1889, Protozoaires: in: Mission scientifique du Cap Horn 1882-1883: v. VI, Zoologie, 3e partie, section L, p. L3-L43, pls. 1-6: Gauthier-Villars (Paris). No additional data available.

Certes, A., ??title: Mem. Academia dei Lincei, v. 21, p. 293. (see Hopkinson 1919). No additional data available.

Chardez, D., 1956, Variations morphologiques et tératologie chez quelques Rhizopodes testacés: Biologisch Jaarboek Dodonaea (Gent), Jaargang, v. 23, p. 265276, pls. 1-5.

Chardez, D., 1956a, Thécamoebiens de la region Verviétoise: Revue Verviétoise d'Histoire Naturelle, v. 13, p. 23-32.

Chardez, D., 1956b, Contribution à la faune Rhizopodique de Belgique I: Revue Verviétoise d'Histoire Naturelle, v. 13, p. 23-32.

Chardez, D., 1957a, Quelques thécamoebiens d'une station muscicole du Congo. No data available.

Chardez, D., 1957b, Thécamoebiens mousses Aériennes: No data available.

Chardez, D., 1957c, Thécamoebiens nouveaux pour la faune Rhizopodique de Belgique: Revue Verviétoise d'Histoire Naturelle, v. 14, p. 13-15.

Chardez, D., 1957d, Contribution à la faune Rhizopodique de Belgique II.

Thécamoebiens de la Fagne: Revue Verviétoise d'Histoire Naturelle, v. 14, p. 62-79. check vol and pages

Chardez, D., 1957e, Rhizopodes de Belgique: Revue Verviétoise d'Histoire Naturelle, v. 13, p. 82-87.

Chardez, D., 1958a, Contribution à la faune Rhizopodique de Belgique III. Thécamoebiens des Ardennes: Revue Verviétoise d'Histoire Naturelle, v. 15, p. 2429, 88-90.

Chardez, D., 1958b, Étude sur les thécamoebiens d'une petite pièce d'eau: Hydrobiologia, v. 10, p. 292-304.

Chardez, D., 1959, Thécamoebiens des terres de Belgique I: Hydrobiologia, v. 14, p. 72-78.

Chardez, D., 1960a, Introduction à l'étude des Thécamoebiens du sol: Bulletin de I'Institut Agronomique et des Stations de Recherches de Gembloux, v. 28, no 2, p. 118-131. 
Chardez, D., 1960b, Étude comparée des Thécamoebiens de trois biotopes dans trois milieux différents: Bulletin de l'Institut Agronomique et des Stations de Recherches de Gembloux, v. 28, no 2, p. 132-138.

Chardez, D., 1960c, Note sur les Thécamoebiens d'Otrange (Hesbaye) et découverte d'un genere nouveau: Annales de la Société Royale de Zoologie de Belgique, v. 91, fasc. 1, p. 39-43.

Chardez, D., 1960d, Sur quelques Thécamoebiens du genre Trinema Dujardin:

Bulletin de l'Institut Agronomique et des Stations de Recherches de Gembloux, v. 28, p. 266-271.

Chardez, D., 1960e, Thécamoebiens terricoles d'une station préhistorique: Revue Verviétoise d'Histoire Naturelle, v. 17, p. 67.

Chardez, D., 1960f, Thécamoebiens des terres Belgiques II: Hydrobiologia, v. 16, p. 197-202.

Chardez, D., 1960g, Étude sur deux Difflugia: Hydrobiologia, v. 16, p. 118-125.

Chardez, D., 1961a, Catalogue des Théamoebiens de Belgique (Protozoa, Rhizopoda testacea): Bulletin de l'Institut Agronomique et des Stations de Recherches de Gembloux, v. 29, no 3-4, p. 269-300.

Chardez, D., 1961b, Sur Difflugia acuminata Ehrenberg (Rhizopoda, Testacea): Bulletin de l'Institut Agronomique et des Stations de Recherches de Gembloux, v. 29, no 3-4, p. 301-308.

Chardez, D., 1961c, Note sur Phryganella acropodia (Hertwig et Less) Hopkinson et sa variété penardi Decloître (Protozoa Rhizopoda): Bulletin de l'Institut Agronomique et des Stations de Recherches de Gembloux, v. 29, no 2, p. 122-124.

Chardez, D., 1961d, Contribution à la faune Rhizopodique de Belgique IV.

Thécamoebiens des régions Campinoises: Revue Verviétoise d'Histoire Naturelle, v. 18, p. 34-37.

Chardez, D., 1961e, Thécamoebiens de la Baraque Fraiture: Biologisch Jaarboek Dodonaea (Gent), Jaargang.

Chardez, D., 1962a, Note sur Euglypha loevis Perty (Protozoa Rhizopoda): Bulletin de I'Institut Agronomique et des Stations de Recherches de Gembloux, v. 30, no 1-2, p. 84-86.

Chardez, D., 1962b, Deux vaeiétés nouvelles de Thécamoebiens, Rhizopoda testacea: Bulletin de l'Institut Agronomique et des Stations de Recherches de Gembloux, v. 30, no 3-4, p. 260-262.

Chardez, D., 1962c, Sur les Thécamoebiens des sols forestiers du Sart-Tilman (Liège): 
Bulletin de I'Institut Agronomique et des Stations de Recherches de Gembloux, v. 30, no 3-4, p. 263-272.

Chardez, D., 1962d, Sur Centropyxis sylvatica (Deflandre) Thomas: Biologische Jaarboek (Gent), v. 30, p. 317-320.

Chardez, D., 1963a, Thécamoebiens des fumiers: Bulletin de l'Institut Agronomique et des Stations de Recherches de Gembloux, v. 31, no 1, p. 17-20.

Chardez, D., 1963b, Thécamoebiens des Hautes Fagnes en Belgique: Revue des Hautes Fagnes, v. 3, p. 142-153.

Chardez, D., 1963c, Documents ichonographiques sur les Thécamoebiens: Roneo chez l'auteur (?), p. 1-21. [NOTE: check name]

Chardez, D., 1964a, Thécamoebiens pélagiques (Protozoa Testacea): Bulletin de I'Institut Agronomique et des Stations de Recherches de Gembloux, v. 32, no 3, p. 299-304.

Chardez, D., 1964b, Thécamoebiens (Rhizopodes testacés): in: Symoens, J.- J.,(ed.): Exploration hydrobiologique du bassin du Lac Bangweolo et du Luapula. Résultats scientifiques, Thécamoebiens, v. 10, fasc. 2, p. 1-77: Cercle Hydrobiologique de Bruxelles (Bruxelles).

Chardez, D., 1964c, Sur la répartition verticale des Thécamoebiens endogé: Bulletin de I'Institut Agronomique et des Stations de Recherche de Gembloux, v. 32, no 1, p. 26-32.

Chardez, D., 1964d, Sur la nutrition de Centropyxis discoides (Penard) Deflandre (Rhizopoda Testacea): Bulletin de l'Institut Agronomique et des Stations de Recherche de Gembloux, v. 312, no 3, p. 305-308.

Chardez, D., 1964e, Thécamoebiens des environs de Waremme (Hesbaye): Revue Verviétoise d'Histoire Naturelle, v.(?)., no 10-12, p. 1-5.

Chardez, D., 1965a, Sur un mode particulier et peu connu de reproduction chez les Thécamoebiens aquatique (Rhizopoda Testacea): Bulletin de l'Institut Agronomique et des Stations de Recherche de Gembloux, v. 33, no 1, p. 25-34.

Chardez, D., 1965b, Kystes de résistance chez Hyalosphenia papilio Leidy (Rhizopoda Testacea): Bulletin de I'Institut Agronomique et des Stations de Recherche de Gembloux, v. 33, no 4, p. 488-490.

Chardez, D., 1965c, Ecologie générale des Thécamoebiens: Bulletin de l'Institut Agronomique et des Stations de Recherche de Gembloux, v. 33, no.(?).., p.307341.

Chardez, D., 1966a, Sur Difflugia bacillifera Penard (Thécamoebien): Revue 
Verviétoise d'Histoire Naturelle, v. 23, no 1-2, p. 15-17.

Chardez, D., 1966b, Note sur quelques Thécamoebiens Eulobosa: Revue Verviétoise d'Histoire Naturelle, v. 23, no 3-5, 4 p.??

Chardez, D., 1966c, Sur la présence en Belgique de Difflugia sarissa Li Sun Taï (Protozoa Rhizopoda Testacea): Revue Verviétoise d'Histoire Naturelle, v. 23, no 912, $3 \mathrm{p}$.

Chardez, D., 1966d, Influence du milieu sur Centropyxis aculeata (Ehrenberg) Stein (Rhizopoda Testacea): Bulletin de l'Institut Agronomique et des Stations de Recherche de Gembloux, v. 1, no 1, p. 13-19.

Chardez, D., 1966e, Note sur quelques Thécamoebiens: Bulletin de l'Institut Agronomique et des Stations de Recherche de Gembloux, v. 1, no 1, p. 20-24.

Chardez, D., 1966f, Études monographiques sur quelques genres de Thécamoebiens (Protozoa Rhizopoda Testacea): Bulletin des Recherches Agronomiques de Gembloux, new series, v. 1, no 2, p. 177-189.

Chardez, D., 1967a, Histoire Naturelle des Protozoaires Thécamoebiens: Les Naturalistes Belges, v. 48, no 10, p. 484-576, pls.1-8.

Chardez, D., 1967b, Remarques sur le genre Phryganella: ?? 4 p. No additional data available.

Chardez, D., 1968, Étude statistique sur l'écologie et la morphologie des Thécamoebiens: Hydrobiologia, v. 32, p. 271-287.

Chardez, D., 1969a, Le genre Phryganella Penard (Protozoa Rhizopoda Testacea): Bulletin des Recherches Agronomiques de Gembloux, new series, v. 4, no 3-4, p. 315-322.

Chardez, D., 1969b, Contribution à la faune thécamoebienne de l'Island (Protozoa Rhizopoda Testacea): Bulletin de l'Institut Royal des Sciences Naturelles de Belgique, v. 45, no 29, p. 1-16, 2 pls.

Chardez, D., 1969c, Notre sur Euglipha (sic!) filifera Penard (Rhizopoda Testacea: Revue Verviétoise d'Histoire Naturelle, v. 26, no 4-6, 4 p.

Chardez, D., 1970, Étude sur Centropyxis aculeata (Ehrenberg) Stein (Protozoa Rhizopoda Testacea): Bulletin des Recherches Agronomiques de Gembloux, new series, v. 5, no 1-2, p. 76-86.

Chardez, D., 1971a, Protozoaires ciliés et Thécamoebiens du système digestif des Bovidés: Bulletin des Recherches Agronomiques de Gembloux, new series, v. 5, no 1-2, p. 15-32.

Chardez, D., 1971b, Étude sur les thécamoebiens des biotopes interstitiels psammons 
littoraux et zones marginales souterraines des eaux douces: Bulletin des Recherches Agronomiques de Gembloux, new series, v. 6, no 3-4, p. 257-268.

Chardez, D., 1973a, Sur Difflugia ventricosa Deflandre 1926 (Rhizopode Thécamoebien): Revue Verviétoise d'Histoire Naturelle, v. 30, no 7-9, p. 57-60, 4 p.

Chardez, D., 1973b, Sur quelques Thécamoebiens d'Islande: Revue Verviétoise d'Histoire Naturelle, v. 30, no 10-12, 4 p.

Chardez, D., 1974a, Sur les thécamoebiens des rhizosphères et des feuilles d'arbre: Bulletin des Recherches Agronomiques de Gembloux, new series, v. 9, no 1, p. 312.

Chardez, D., 1974b, Thécamoebiens du Djebel Marra, massif volcanique au Soudan: Revue Verviétoise d'Histoire Naturelle, v. 31, no 1-3, p. 1-8.

Chardez, D., 1975a, Thécamoebiens des Lichens: Revue Verviétoise d'Histoire Naturelle, v. 32, no 7-9, 7 p.

Chardez, D., 1975b, Quelques Thécamoebiens de la région de Butare (Zaïre): Revue Verviétoise d'Histoire Naturelle, v. 32, no 7-9, 2 p.

Chardez, D., 1976a, Addition au catalogue des thécamoebiens de Belgique: Revue Verviétoise d'Histoire Naturelle, v. 33, no 7-9, p. 47-48.

Chardez, D., 1976b, Thécamoebiens récoltés dans un prélèvement de mousses épigées: Revue Verviétoise d'Histoire Naturelle, v. 33, no 4-6, p. 25-27.

Chardez, D., 1977, Thécamoebiens du mésopsammon des plages de la mer du Nord: Revue Verviétoise d'Histoire Naturelle, v. 34, no 4-6, p. 18-34.

Chardez, D., 1978a, Note thécamoebologiques: Revue Verviétoise d'Histoire Naturelle, v. 35, no 1-3, p. 6-9.

Chardez, D., 19781b, Colonisation par les Protozoaires des surfaces immergées dans an aquarium: Les Naturalistes Belges (Bruxelles), v. 59, p. 132-137.

Chardez, D., 1978c, Hyalosphenia savoiei sp. nov. (Thécamoebien mésopsammique): Revue Verviétoise d'Histoire Naturelle, v. 35, no 7-9, 2. p.

Chardez, D., 1979, Variations chez Centropyxis aerophila sphagnicola Deflandre: Revue Verviétoise d'Histoire Naturelle, v. 36, no 1-3, 4 p.

Chardez, D., 1980, Thécamoebiens de l'étang de Thommen (Protozoa Rhizopoda Testacea): Les Naturalistes Belges, v. 61, p. 88-91.

Chardez, D., Gaspar, C., 1976, Thécamoebiens aquatiques du domaine des Epioux (Ardenne, Belgique) (Protozoa, Rhizopoda, Testacea): Biologische Jaarboek, v. 44, pp. $86-100$ 
Chardez, D., 1986, Thécamoebiens des plages de la Mer du Nord en Angleterre: Acta Protozoologica, v. 25, pp. 375-378

Chardez, D., 1987, Contribution a la connaissance des thecamoebiens des depots lacustres: Acta Protozoologica, v. 26, pp. 59-62

Chardez, D., 1990, Contribution a la connaissance des Thécamoebiens aquatiques du Tyrol allemand (Rhizopoda, Testacea): Acta Protozzologica, v. 29, pp. 153-156

Chardez, D., 1990, Thécamoebiens (Rhizopoda, Testacea) des millieux anisooligohydriques mousses et lichens: Acta Protozzologica, v. 29, pp. 147-152

Chardez, D. 1991, Note sur Difflugia humilis sp. n. (Protozoa: Rhizopoda: Testacea): Acta Protozoologica, v. 30, pp. 45-47

Chardez, D., 1994, Contribution á l'étude biogeographique de Centropyxis carinata Chardez: Acta Protozoologica, v. 33, pp. 195-198

Chardez, D., Beyens, L., de Bock, P., 1988, Centropyxis gasparella sp. nov. and Parmulina louisi sp. nov., new testate amoebae from the Canadian High Arctic (Devon Island, NWT): Archiv für Protistenkunde, v. 136, pp. 337-344

Chardez, D. and Decloître, L., 1973, Nota sistematica sobre la validez de dos especies de Tecamebianos: Difflugia oblonga Ehrenberg y Difflugia pyriformis Perty: Physis (Buenos Aires), Sección B, v. 32, no 85, p. 359-365, pl. 1.

Chardez, D., Delecour, F., 1970: Premières observations sur les thécamoebiens des sols forestiers ardennais: Revue d'Ecologie et de Biologie du Sol, v. 7, no 1, p. 3334.

Chardez, D., Delecour, F., Weissen, F., 1972, Evolution des populations thécamoebiennes de sols forestiers sous l'influence de fumure artificielles: Revue d'Ecologie et de Biologie du Sol, v. 9, no 2, p. 185-196.

Chardez, D., Gaspar, Ch., 1976, Thécamoebiens aquatiques du domaine des Epioux (Ardenne, Belgique) (Protozoa Rhizopoda Testacea): Biologish Jaarb (Gent), v. 44, p. 86-100.

Chardez, D., Hellebaut, R., 1978, Effet de la pollution sur la population thécamoebiennes d'un étang: Revue Verviétoise d'Histoire Naturelle, v. 35, no 4-6, p. 29-32.

Chardez, D., Leclercq, J., 1963, Variabilité des populations d’Euglypha strigosa (Ehrenberg) Leidy en fonction de l'habitat (Rhizopoda Testacea): Bulletin de l'Institut Agronomique et des Stations de Recherche de Gembloux, v. 31, no 1, p. 21-27.

Chardez, D., Krizelj, S., 1970, Recherches sur l'écosystème forêt. Sér. B.La chênaie mélangé calcicole de Virelles. Contribution no 22. Protozoaires thécamoebiens et ciliés 
du sol: Bulletin de l'Institut Royal des Sciences Naturelles de Belgique, v. 46, no 12, p. 1-17.

Chardez, D., Mommaerts, F., Tahon, J., 1972, Recherches sur l'écosystème forêt. Sér. B: la chênaie mélangée calcicole de Virelles-Blaimont. Contribution no 38. Thécamoebiens des dépôts épiphytes arboricoles: Bulletin de l'Institut Royal des Sciences Naturelles de Belgique, v. 48, Biologie, no 2, p. 1-7, pl. 3.

Chardez, D., Thomas, R., 1980, Thécamoebiens du mésopsammon des plages de Lacanau et Leporge-Océan (Gironde, France) (Protozoa Rhizopoda Testacea): Acta Protozoologica, v. 19, no 3, p. 277-286.

Charman, D.J. (1997) Modelling hydrological relationships of testate amoebae (Protozoa: Rhizopoda) on New Zealand peatlands. Journal of the Royal Society of New Zealand, 27 465-483.

Charman, D.J., Warner, B.G. (1992) Relationship between testate amoebae (Protozoa:Rhizopoda) and the micro-environmental parameters on a forested peatland in northeastern Ontario. Canadian Journal of Zoology, 70 2474-2482.

Charman, D.J., Warner, B.G. (1997) The ecology of testate amoebae (Protozoa: Rhizopoda) in oceanic peatlands in Newfoundland, Canada: modelling hydrological relationships for palaeoenvironmental reconstruction. Écoscience, 4 555-562.

Charman, D.J., Roe, H.M. and Gehrels, W.R. (1998) The use of testate amoebae in studies of sea-level change: a case study from the Taf estuary, South Wales, UK. The Holocene, 8 209-218.

Charret, R., 1962, L'exuviation chez Hyalosphenia papilio (Rhizopode Testacé):

Compt. rend. Acad. Sci., Paris, v. 254, no 4, p. 730-732.

Charret, R. and Vivier, E., 1964, Sur l'ultrastructure d'Arcella polypora (Rhizopode testacé): in: Titlbach, M. (ed.): Electron Microscopy 1964-Proceedings of the Third European Regional Conference held in Prague, August 26- September 3, 1964, v. B, p. 187-188: Publishing House of the Czechoslovak Academy of Sciences (Prague).

Chatton, É, 1925, Pansporella perplexa, Amoebien à spores protégées parasite des Daphnies. Réflexions sur la biologie et la phylogénie des Protozoaires: Annales des Sciences Naturelles, Zoologie, 10 sér., v. 8, no 1, p. 5-85, pl. 1.

Chen, J.C., Shieh, Y.C., 1981, Primary investigation on plankton distribution of several lakes and reservoirs in Taiwan: Journal, Fisheries Society of Taiwan, v. 8, pp. 70-88 (in Chinese).

Chodat, R., 1920, Algues de la région du Grand St-Bernard: Bulletin de la Société de Botanique de Genève, sér. 2, v. 12, p. 293-305.

Churchill, E. P. Jr., 1922, The effects of so-called conjugation in shelled rhizopods: 
American Naturalist, v. 55 , p. $466-470$

Cicak, A., Mclaughlin, J. J. A., Wittenberg, J. B., 1963, Oxygen in the gas vacuole of the rhizopod protozoan, Arcella: Nature, v. 199, p. 983-985.

Cienkowsky, L., 1875, Ueber einige Rhizopoden und verwandte Organismen: Archiv für Mikroskopische Anatomie, v. 12, p. 15-50, pls. 4-8.

Claparède, E., Ueber Actinophrys eichhornii, Archiv für Anatomie, Physiologie und wissenschaftliche Medicin (Berlin), p. 398-419, pl. 15.

Claparède, E., Lachmann, J., 1858 - 1859 - 1861, Études sur les infusoires et les rhizopodes: Mémoires de I'Institut National Genevois, v. I, v. 5 (1858), p. 1-260, pls. 1-24; v. I, v. 6 (1859), p.261-482; v. II, v. 7 (1861), p. 1-291, pls. 1-13.

Claus, C., 1872, Grundzüge der Zoologie: Marburg and Leipzig, p. 1-1170.

Claus, C., 1884a, Traité de Zoologie: Paris. pages??

Claus, C., 1884b, Elementary text-book of Zoology; v. 1, London. ??pages.

Claus, C., 1887, Lehrbuch der Zoologie: p. I-XII, 1-886, N. G. Elwert (Marburg).

Claus, C., Grobben, K., 1905, Lehrbuch der Zoologie: Marburg in Hessen, p. 1-955.

Claus, C., Grobben, K., 1905, Lehrbuch der Zoologie: Marburg in Hessen:

Elwert'sche Verlagsbuchhandlung: ??pages. 


\section{COC-DAN}

Cockerell, T. D. A., 1907, The protozoa of the University Campus: University of Colorado Studies (University of Colorado, Boulder), Series A, v. 4, no 4, p. 261264.

Cockerell, T. D. A., 1909a, Notes on Protozoa: University of Colorado Studies (University of Colorado, Boulder), Series A, v. 6, no 4, p. 305-307.

Cockerell, T. D. A., 1909b, New names for two genera of Protozoa: Zoologischer Anzriger, v. 34, p. 565.

Cockerell, T. D. A., 1911a, The fauna of Boulder County, Colorada: University of Colorado Studies (University of Colorado, Boulder), Series A, v. 8, no 4, p. 227 256.

Cockerell, T. D. A., 1911b, The nomenclature of the Rhizopoda: Zoologischer Anzeiger, v. 38, p. 136-137.

Cockerell, T. D. A., 1930, Siliceous shell of Protozoa: Nature, v. 125, p. 975.

Thechamoebians (?)

Cohn, F., 1853, Beiträge zur Entwickelungsgeschichte der Infusorien: Zeitschrift für wissenschaftliche Zoologie (Leipzig), v. 4, no 3, p. 253-281, pl. 13.

Cole, T., 1853, List of Infusorial objects found chiefly in the neighbourhood of Salem, Mass: Proceedings of the Essex Institute, v. 1, p. 33-48.

Collin, B., 1914, Notes Protistologiques: Archives de Zoologie expérimentale et générale, v. 54, ??p. 85-97.

Collins, E.S., McCarthy, F.M.G., Medioli, F.S., Scott, D.B., Honig, C.A., 1990, Biogeographic distribution of modern thecamoebians in a transect along the eastern North American coast. In .Proceedings of the NATO Advanced Study Institute on Paleoecology, biostratigraphy, paleoceanography and taxonomy of agglutinated foraminifera; Hemleben, C., Kaminski, M.A., Kuhnt, W., Scott, D.B., (eds.), NATO ASI Series. Series C: Mathematical and Physical Sciences, v. 327, pp. 783-792, D. Reidel Publishing Company. Dordrecht-Boston, International.

Collins, E.S., Schafer, C.T , 1987, Environmental impacts on the foraminifera and thecamoebians of the upper Saguenay Fiord, Quebec, Canada: Abstract, Geological Society of America, Boulder, CO, United States. 1987, v. 19, pp. 624

Colom, G., 1982, Notas paleontologicas y estratigraficas (referentes a Mallorca e lbiza): Boletin de la Sociedad de Historia Natural de Baleares, v. 26, pp. 195-206.

Conn, H. W., 1904, A preliminary report on the Protozoa of the fresh waters of Connecticut: State of Connecticut, State Geological and Natural History Survey 
Bulletin no 1, p. 5-19, pls. 1-5.

Conrad, W., 1942, Flagellates, Algues et Thécamoebiens d'Ardenne: Bulletin du Musee Royal d'Histoire Naturelle de Belgique, v. 18, p. 1-12.

Constantin, P., Godeanu, S. And Negrea, S., 1978, Studiul calitativ si cantitativ al faunei din mlastina Benii V (Subcarpatii de curbura): Studii si Cercetari de Biologie, Seria Biologie Animala (Bucuresti), v. 30, p. 149-153.

Coppa, A., 1921, Ricerche sui Protozoi del terreno e delle acque ticinesi: Staz. Sper. Agrarie Ital. (Modena), v. 54, p. ??.

Coppa-Patrini, A., 1928, I Rizopodi del Lario: Atti della Società Italiana di Scienze Naturali (Milano), v. 67, p. ??.

Corbett, S. A., 1973, An illustrated introduction to the Testate Rhizopods in Sphagnum, with special reference to the area around Malham Tarn, Yorkshire: Field Studies, v. 3, no 5, p. 801-838, pls.1-2.

Cosmovici, N. L., 1912, Contribution à l'étude de la faune protozoaire de la Roumanie: Bull. Soc. Zool. France, v. 37, p. 205-211.

Couteaux, M.M., 1967, Une technique d'observation des Thécamoebiens du sol pour l'estimation de leur densité absolue: Revue d'Ecologie et de Biologie du Sol, v. 4, p. 593-596.

Couteaux, M.M., 1969a, Étude de la communauté de Thécamoebiens d'une chênaire à luzule (Moyenne-Belgique): Comptes Rendus des Séances de l'Académie des Sciences, Paris, Sér. D, v. 269, p. 335-338.

Couteaux, M.M., 1969b, Thécamoebiens muscicoles de Gaume et de MoyenneBelgique: Revue d'Ecologie et de Biologie du Sol, v. 6, no 4, p. 413-428.

Couteaux, M.M., 1970, Thécamoebiens des sols forestiers: recensement quantitatif pour une étude écologique: Journal of Paleontology, v. 17 (suppl.), p.33.

Couteaux, M.M., 1972, Distribution des Thécamoebiens de la litière et de l'humus de deux sols forestiers d'humus brut: Pedobiologia, v. 12, no 4, p. 237-243.

Couteaux, M.M., 1973, Ecologie des Thécamoebiens de quelques humus bruts forestiers. L'espèce dans la dynamique de l'équilibre: Thèse dans d'Etat, Université de Paris VI: p. 1-311.

Couteaux, M.M., 1974, Dynamique des Thécamoebiens dans trois sols forestiers acides: in: DePuyorac, P. and Grain, J. (eds.): Actualités Protozoologiques, v. 1. Résumé des discussions des Tables Rondes de 4e Congrès International de Protozoologie, 2-9 September 1973, Clermont-Ferrand, France, p. 359: Université de Clermont (Clermont-Ferrand). 
Couteaux, M.M., 1975a, Estimation quantitative des Thécamoebiens édaphiques par rapport à la surface du sol: Comptes rendus des Séances de l'Academie des Sciences, Paris, Sér. D, v. 281, p. 739-741.

Couteaux, M.M., 1975b, Quelques aspects des relations entre les Thécamoebiens et les sols: Revue d'Écologie et de Biologie du Sol, v. 12, no 1, p. 45-55.

Couteaux, M.M., 1975c, Écologie des Thécamoebiens de quelques humus bruts forestiers: l'espèce dans la dynamique de l'équilibre: Revue d'Écologie et de Biologie du Sol, v. 12, no 2, p. 421-447.

Couteaux, M.M., 1976a, Dynamisme de l'équilibre des Thécamoebiens dans quelques sols climaciques: Mémoires du Muséum National d'Histoire Naturelle, nouvelle sér: Sér. A, Zoologie, v. 96, p. 1-183, 2 plates.

Couteaux, M.M., 1976b, Modification de la faune thécamoebien sous l'effet d'un incendie de for136t en région subméditerranéenne: Compt. Rend. Acad. Sci., Paris, (Sér. D), v. 282, no 9, p. 925-928.

Couteaux, M.M., 1976c, Le peuplement Thécamoebien du sol et la nature de l'eau disponible: Bulletin d'Ecologie (Brunoy), v. 7, no 2, p. 197-206.

Couteaux, M. M., 1976d, Étude quantitative des Thécamoebiens d'une savane à Hyparrhenia à Lamto (Côte-d'Ivoire): Protistologica, v. 12, no 4, p. 563-570.

Couteaux, M.M., 1977, Reconstitution d'une nouvelle communauté Thécamoebienne dans la litière d'une forêt incendiée en région subméditerranéenne: Ecologial Bulletins, Swedish Natural Science Research Council (Statens Naturvetenskapliga Forskningsraad, Ekologi Kommitten) (Stockholm), no 25, p. 102-108.

Couteaux, M.M., 1978a, Essai de taxonomie numérique du genre Euglypha: Journal of Protozoology, v. 25, no 3, part 1, p. 50A.

Couteaux, M.M., 1978b, Quelques Thécamoebiens des sols du Japon: Revue d'Ecologie et de Biologie du Sol, v. 15, no 1, p. 119-128.

Couteaux, M.M., 1978c, Étude quantitative des Thécamoebiens édaphiques dans une savane à Loudetia à Lamto (Côte d'Ivoire): Revue d'Ecologie et de Biologie du Sol, v. 15 , no 3, p. 401-412.

Couteaux, M.M., 1979, L'effet de la déforestation sur le peuplement thécamoebien en Guyane française: étude préliminaire: Revue d'Ecologie et de Biologie du Sol, v. 16, no 3, p. 403-413.

Couteaux, M.M., 1981, Relations trophiques chez les Protozoaires du sol: in: Dryl, S., Kazubski, S. L. and Ploszaj, J. (eds.), Progress in Protozoology. Abstracts of papers submitted to VI International Congress of Protozoology, Warazawa, 
Poland, July 5-11, 1981, p. 64: (Warszawa).

Couteaux, M.M., Chardez, D., 1981, Thécamoebiens édaphiques et muscicoles de Guyane Française: Revue d'Écologie et de Biologie du Sol, v. 18, no 2, p. 193-208.

Couteaux, M.M., Jeanson, C. Y., 1977, Essai d'analyse élémentaire de la thèque de Trigonopyxis arcula (Leidy) Penard (Rhizopoda, Testacea): Ecologial Bulletins, Swedish Natural Science Research Council (Statens Naturvetenskapliga Forskningsraad, Ekologi Kommitten) (Stockholm), no 25, p. 164-172.

Couteaux, M.M., Munsch, A., 1978, Thécamoebiens de mangroves: Revue d'Ecologie et de Biologie du Sol, v. 15, no 3, p. 391-399.

Couteaux, M.M., Munsch, A., Ponge, J.-F., 1979, Le genre Euglypha: essai de taxinomie numérique: Protistologica, v. 15, no 4, p. 565-579.

Craig, T., 1897, ?? title: American Monthly Microscopical Journal, v. 18, p. ?.

Cumming, J.D., 1992, The physical and chemical characteristics of Oromocto Lake, New Brunswick, and the use of arcellaceans as geochemical indicators, Department of Earth Science, Carleton University Honour's Thesis, $35 \mathrm{p}$.

Cuneo, G., 1890, Ricerche sui Protisti della acque di Rapallo: Bollettino Scientifico (Pavia), v. 3, anno 12, no 4, p. 140-149.

Cunha, A. M. da, 1913, Contribuiço para o conhecimento da fauna de protozoarios do Brazil. Beitraege zur Kenntnis der Protozoenfauna Brasiliens: Mem. Inst. Oswaldo Cruz, v. 5, no 2, p. 101-122, pls. 9, 10.

Cushman, J. A.,1906a, Fresh-water Rhizopods of Nantucket: American Naturalist, v. 40, p. 371-373.

Cushman, J. A., 1906b, A preliminary study of the finer structure of Arcella: American Naturalist, v. 40, p. 797-802.

Cushman, J. A., 1930, The Foraminifera of the Chaoctawhatchee Formation of Florida: Florida State Geological Survey Bulletin, v. 4, p. 1-63, pls. 1-12.

Cushman, J. A., Bronnimann, P., 1948a, Some new genera and species of Foraminifera from brackish water of Trinidad: Cushman Laboratory for Foraminiferal Research, Contributions, v. 24, no 1, p. 15-21, pls. 3-4.

Cushman, J. A. Bronnimann, P., 1948b, Additional new species of arenaceous Foraminifera from shallow waters of Trinidad: Cushman Laboratory for Foraminiferal Research, Contributions, v. 24, no 2, p. 37-42, pls. 7-8.

Cushman, J. A., Cahill, E. D., 1933, Miocene Foraminifera of the Coastal Plain of the Eastern United States: U.S. Geological Survey, Professional Paper 175-A, p. 1-50, pls. 1-13. 
Cushman, J. A., Henderson, W. P. 1905, Freshwater Rhizopods from the White Mountain region of New Hampshire: American Naturalist, v. 39, p. 147-155.

Cushman, J. A., McCulloch I., 1939, A report on some arenaceous Foraminifera: Allan Hancock Pacific Expeditions, v. 6, no 1, p. i-vi, 1-113, pls. 1-12.

Cushman, J.A., McCulloch , I., 1948, Three new names for Recent Pacific Foraminifera: Cushman Laboratory for Foraminiferal Research, Contributions, v. 24, no 3, p. 76.

Daday, J., 1885, Adatok a Balaton-tó faunájának ismeretéhez: Értes. math.termész. Akad. Budapest, v. 3, p. 160-164.

Daday, J., 1891, Beiträge zur mikroskopischen Süsswasserfauna Ungarns: Természetragzi Füzetek (Naturhistorische Hefte, herausgegeben vom Ungarischen National-Museum) (Budapest), v. 14, p. 107-123, pl. 1.

Daday, J., 1892, A mezöségi tavak mikrospópos állatvilagé: Természetragzi Füzetek (Naturhistorische Hefte, herausgegeben vom Ungarischen National-Museum) (Budapest), v. 15, p. 1-31, pl.1.

Daday, J. 1892, Die mikroscopische Thierwelt der Mezoseger Teich: Termeszetrajzi Fuzetek, v. 15, p. 166-207.

Daday, J., 1897, Beiträge zur Kenntniss der Mikrofauna der Tatra-Seen: Természetragzi Füzetek (Naturhistorische Hefte, herausgegeben vom Ungarischen National-Museum) (Budapest), v. 20, p. 149-196.

Daday, J., 1898, Mikroskopische Süsswasserthiere aus Ceylon: Anhang Természetrajzi Füzetek, v. 21, p. 1-123.

Daday, J., 1901, Mikroskopische Süsswasserthiere in Horváth, (ed.), Zoologische Ergebnisse Zichy, v. 2, p. 375-470, pls. 14-28.

Daday, J., 1903, Mikroskopische Süsswasserthiere aus Kleinasien: Sitzungberichte der Akademie der Wissenschaftlichen in Wein, v. 112, no 1, p. 139-167.

Daday, J., 1903 or 4, Mikroskopische Süsswasserthiere der Umgebung des Balaton: Zoologische Jahrbuch (Systematic), v. 19, no 1, p. 37-98.

Daday, J., 1904, Mikroskopische Süsswasserthiere aus Turkestan: Zoologische Jahrbuch (Systematic), v. 19, no ??, p. 469-553.

Daday, J., 1905, Untersuchungen über die Süsswasser-Mikrofauna Paraguays: Zoologica, v. 18 (part 44), p. 1-374, pls. 1-23.

Daday, J., 1906, Mikroskopische Süsswasserthiere aus der Mongolei: Math.Termész értes., v. 24, p. 34-77. 
Daday, J., 1907a, ??title: Math. Termész értes., v. 25, p. 402-420.

Daday, J., 1907b, Plankton-Tiere aus dem Victoria-Nyanza: Zoologische Jahrbuch (Systematic), v. 25, no 2, p. 245-262.

Daday, J., 1910a, Beiträge zur Kenntnis der Mikrofauna des Nils: Sitzungsberichte der Akadenie der Wissenschaften in Wien, v. 119, no 1, p. 537-589.

Daday, J., 1910b, Untersuchungen über die Süsswasser-Mikrofauna Duetsch-OstAfrikas: Zoologica ??, v. 23 (part 59), p. 1-314, pls. 1-18.

Daday, J., 1910c, ??title: in editor, Deutsch-Ost-Afrika, Band 4, part 23, ??pages.

Dampf, G., 1913, Die faunistische Erforschung der moore Ostpreussens: Schriften der Physikalische-Okonomischen Gesellschaft zu Konigsberg, v. 54, p. 95-109.

Dangeard, P. A., C., 1900, ??title: Le Botaniste, v. 7, ??pages.

Dangeard, P. A. C., 1903a, Contribution à l'étude des Diplozoaires: Comptes rendus des Séances de I'Académie des Sciences, Paris, v. 136, p. 769-771.

Dangeard, P. A. C., 1903b, Contribution à l'étude des Diplozoaires: Le Botaniste, v. 9., no 1, p. 25-28.

Dangeard, P. A. C., 1904, Contribution à l'étude des Diplozoaires. L' Arcella vulgaris: Microgr. prép., v. 12, p. 84-86. 


\section{DEB-DEC}

Debenay, J.P., 1987, Foraminiferes et thecamoebiens de l'estuaire hyperhalin du fleuve Casamance (Senegal): Revue D’ Hydrobiologie Tropicale, v. 20, pp. 233-256.

Debenay, J.P., Diouf, P.S, 1986, Zonation de la Casamance basee sur les peuplements. Comparaison avec d'autres zonations ecologiques in L'estuaire de la Casamance: environnement, peche, socio economie. le-Reste,-Fontana, L., Samba, A. (eds.), pp. 85-90.

Decloître, L., 1947a, Rhizopodes de la Station de Tanaf: La Feuille des Naturalistes, Bulletin de la Société des Naturalistes Parisienes, new series, v. 2, no 9-10, p. 9194.

Decloître, L., 1947b, Faune Rhizopodique des marécages de Tiaroye: La Feuille des Naturalistes, Bulletin de la Société des Naturalistes Parisienes, new series, v. 5, no 4, p. 38.

Decloître, L., 1948, Matériaux pour une faune Rhizopodique d'A. O. F.: Bulletin de I'Institut Français d'Afrique Noire, v. 10, p. 235-284.

Decloître, L., 1949a, Faune Rhizopodique des marécages de M'Baqouar: La Feuille des Naturalistes, Bulletin de la Société des Narutalistes Parisienes, new series, v. 4, p. 32-36.

Decloître, L., 1949b, Matériaux pour une faune Rhizopodique d'A. O. F.: Bulletin de I'Institut Français d'Afrique Noire, v. 11, no 3, p. 281-301.

Decloître, L., 1950, Étude sur les Rhizopodes: La Feuille des Naturalistes, Bulletin de la Société des Naturalistes Parisienes, new series, v. 5, no 5-6, p. 41-46.

Decloître, L., 1951a, Matérieaux pour une faune rhizopodique d'A.O.F. (Suite). Faune du Lac Tamna (Sénégal): Bulletin de l'Institut Français d'Afrique Noire, v. 13, no I, p. 87-108.

Decloître, L., 1951b, Rhizopods de la station de Tairoye (Sénégal) in: Trabalhos da 2 Conferência Internacional Sôbre Africa ocidental, v. 3, p. 99-102, (Bissau).

Decloître, L., 1953a, Recherches sur les Rhizopodes Thécamoebiens d'A.O.F.: Mémoires de I'Institut Français d'Afrique Noire, no 31, p. 1-249.

Decloître, L., 1953b, Rhizopodes de l'Afrique orientale anglaise: La Feuille des Naturalistes, Bulletin de la Société des Naturalistes Parisienes, new series, v. 11, no 4 , p. ??.

Decloître, L., 1954a, Répartition biogéographie de quelques Nebela: CR. Soc. Biogéograph, no 271, p. ???. 
Decloître, L., 1954b, Mission A. Villiers au Togo et au Dahomey (1950), XXIII Rhizopodes: Bulletin de I'Institut Français d'Afrique Noire, v. 16, no 1, sér.A, p. 89125.

Decloître, L., 1954c, Biostatistique, biogéographie et Thécamoebiens d'A.O.F.: Bulletin de l'InstitutFrançais d'Afrique Noire, v. 16, no 2, sér. A, p. ??.

Decloître, L., 1954d, Contribution à l'étude du peuplement de la Mauritanie: Bulletin de I'Institut Français d'Afrique Noire, v. 16, no 2, sér. A, p. 398-413.

Decloître, L., 1954e, Biométrie et Thécamoebiens d'A.O.F.: Bulletin de l'Institut Français d'Afrique Noire, v. 16, no 3, sér. A, p??.

Decloître, L., 1954f, Thécamoebiens d'une source d'eau chaude de Guinée: Bulletin de I'Institut Français d'Afrique Noire, v. 16, no.., sér. A, p. 829-833.

Decloître, L., 1955a, Rhizopodes Thécamoebiens du Vénézuéla: Hydrobiologia, v. 7, p. 325-372.

Decloître, L., 1955b, Speleologia Africana. Thécamoebiens de la grotte des Singes à Ségéa (Guinée): Bulletin de I'Institut Français d'Afrique Noire, v. 17, no., sér. A, p. 989-1019.

Decloître, L., 1956a, Les Thécamoebiens de l'Eqe (Groenland): Expéditions Polaires Françaises - Missions Paul-Emile Victor - VIII - p. 100, pls. 1-8; Actualités Scientifiques et Industrialles, no 1242, Hermann and Co. (Paris).

Decloître, L., 1956b, Matériaux pour une faune thécamoebiennes de Madagascar: Mémoires de l'Institut Scientifique du Madagascar, (A), v. 11, p. 1-31.

Decloître, L., 1956c, Rhizopodes thécamoebiens: Mémoires de l'Institut Français d'Afrique Noire, no 48, Le Parc National du Niokolo-Koba, fasc. 1, p. 233-258.

Decloître, L., 1958, Thécamoebiens récoltés au Cameroun: Bulletin de l'Institut Français d'Afrique Noire, v...., no 4, sér. A, p. 139-144.

Decloître, L., 1959, Sur quelques thécamoebiens du Bassin Parisien: Cahiers des Naturalistes, Bulletin des Naturalistes Parisiens, new series, v. 15, no 2, p. 33-34.

Decloître, L., 1961a, Matériaux pour une faune thécamoebienne du Maroc. Prèmiere note: Bulletin de la Société des Sciences Naturelles et Physiques du Maroc, v. 41, p. 117-119.

Decloître, L., 1961b, Matériaux pour une faune thécamoebienne du Maroc. Deuxième note: Bulletin de la Société des Sciences Naturelles et Physiques du Maroc, v. 41, p. 121-136.

Decloître, L., 1962a, Le genre Euglypha Dujardin: Archiv für Protistenkunde, v. 106, p. 51-100. 
Decloître, L., 1962b, Trinema lineare var.terricola nov. var. (Thécamoebiens):

Internationale Revue der gesamten Hydrobiologie, v. 47, no 1, p. 157-163.

Decloître, L., 1962c, Sur quelques thécamoebiens récoltés en Norvège:

Hydrobiologia (?), v. 19, no 2, p. 179-182.

Decloître, L., 1962d, Thécamoebiens de la XIII Expédition Antartique Française:

Territoires des terres australes et antartiques françaises, publication no 259, p. 147.

Decloître, L., 1964a, Rectification: Schoenbornia nov. gen. (Testacea): Limnologica, v. 2, no 3, p. 336.

Decloître, L., 1964b, Matériaux pour une faune thécamoebienne du Maroc: Bulletin de la Société des Sciences Naturelles et Physiques du Maroc, v. 44, no 1,2, p. 121136.

Decloître, L., 1965a, Contribution à la faune du Congo (Brazzaville?). Mission A. Dexarpentries et A. Villiers. III. Rhizopodes Thécamoebiens: Bulletin de l'Institut Français d'Afrique Noire, v. 27, no 1, sér. A, p. 165-184.

Decloître, L., 1965b, Amoebida testacea (Rhizopoda) in Bertelsen, E., Einarsson, H., Fridriksson, Gudmundsson, F., Spärck, Rand Tuxen, S. L. (eds.), The Zoology of Iceland, v. 2, part 1, p. 1-58: Ejnar Mu nksgaard (Copenhagen and Reykjavík).

Decloître, L., 1965c, Thécamoebiens recueillis dans les sols aériens des palmiers de Quarzazate: Bulletin de la Société des Sciences Naturelles et Physiques du Maroc, v. 45 , p. $17-28$.

Decloître, L., 1965d, Thécamoebiens nouveaux du Maroc: Société des Sciences Naturelles et Physiques du Maroc, v. 31, p. 33-38.

Decloître, L., 1965e, Catalogue des thécamoebiens reconnus au Maroc: Bulletin de la Société des Sciences Naturelles et Physiques du Maroc, v. 45, no 1,2, p. 17-27. same page numbers as $1965 \mathrm{c}$.

Decloître, L., 1966a, Thécamoebiens d'une cressonière naturelle dans une seghuia de l'Ecole Nationale d'Agriculture de Meknès (Maroc): Internationale Revue der gesamten Hydrobiologie, v. 47, no 2, p. 307-314.

Decloître, L., 1966b, Comment compter le nombre de Thécamoebiens dans une ricolte: Limnologica, v. 4, no 3, p. 489-492.

Decloître, L., 1968, Les Thécamoebiens du Grand Lautien (Var): Annales de la Société des Sciences Naturelles et d'Archéologie de Toulon et du Var, no 20, p. 182-190.

Decloître, L., 1969a, Thécamoebiens du sol dans le Var. Pinède de Six-Fours-la-Plage: 
Annales de la Société des Sciences Naturelles et d'Archéologie de Toulon et du Var, no 21, p. 150-153.

Decloître, L., 1969b, Thécamoebiens bryomadicoles dans le Var. Récolte de SixFours-la-Plage: Annales de la Société des Sciences Naturelles et d'Archéologie de Toulon et du Var, no 21, p. 154-158.

Decloître, L., 1969c, Contribution à la faune de l'Iran. Rhizopodes thécamoebiens: Bulletin de Muséum National d'Histoire Naturelle (2e série) (Paris), v. 41, no 1, p. 363-371.

Decloître, L., 1970a, Thécamoebiens de liches et mousses, région Méditerranéenne: Annales de la Société des Sciences Naturelles et d'Archéologie de Toulon et du Var, no 22, p. 145-152.

Decloître, L., 1970b, Thécamoebiens du sol dans le Var (2me note). Étude d'une pinède à Six-Fours-la-Plage: Annales de la Société des Sciences Naturelles et d'Archéologie de Toulon et du Var, no 22, p. 153-158.

Decloître, L., 1970c, Sue quelques Thécamoebiens, dont deux nouveaux, récoltés en Haute-Savoie: Cahiers des Naturalistes, Bulletin des Naturalistes Parisiens, nouvelle série, v. 26, p. 55-56.

Decloître, L., 1970d, Thécamoebiens d'un bassin artificiel dans une courde l'E.N.A. de Meknès: Hydrobiologia, v. 67, p. 97-102.

Decloître, L., 1971, Thécamoebiens de Solliés-Pont (Var): Annales de la Société des Sciences Naturelles et d'Archéologie de Toulon et du Var, no 23, p. 161-165.

Decloître, L., 1972a, Thécamoebiens du Var (Suite): Annales de la Société des Sciences Naturelles et d'Archéologie de Toulon et du Var, no 24, p. 164-171.

Decloître, L., 1972b, Notes sur les Thécamoebiens: Travaux du Laboratoire d'Hydrobiologie (Grenoble), no 63, p. 253-256. check serial, vol, no?

Decloître, L., 1973a, Thécamoebiens muscicoles du Var: Annales de la Société des Sciences Naturelles et d'Archéologie de Toulon et du Var, no 25, p. 149-155.

Decloître, L., 1973b, Thécamoebiens des sols cultivés dans le Var: Annales de la Société des Sciences Naturelles et d'Archéologie de Toulon et du Var, no 24, p. 147-148.

Decloître, L., 1973c, Thécamoebiens des lles Galapagos: Ciencia y Naturaleza, v. 19, no 1, p. 11-20.

Decloître, L., 1974a, Thécamoebiens des sols cultivés dans le Var (10 suite): Annales de la Société des Sciences Naturelles et d'Archéologie de Toulon et du Var, no 26, p. 3-4. 
Decloître, L., 1974b, Thécamoebiens de sol cultivé en mimosas dans le Var: Annales de la Société des Sciences Naturelles et d'Archéologie de Toulon et du Var, no 26, p. 5-7.

Decloître, L., 1974c, Thécamoebiens de sol cultivé en figuier dans le Var: Annales de la Société des Sciences Naturelles et d'Archéologie de Toulon et du Var, no 26, p. 9-10.

Decloître, L., 1974d, Thécamoebiens de sol cultivé en olivier dans le Var: Annales de la Société des Sciences Naturelles et d'Archéologie de Toulon et du Var, no 26, p. 11-12.

Decloître, L., 1974e, Thécamoebiens des mousses terrestres dans le Var: Annales de la Société des Sciences Naturelles et d'Archéologie de Toulon et du Var, no 26, p. 13-15.

Decloître, L., 1974f, Thécamoebiens de sols cultivés en amandiers dans le Var: Annales de la Société des Sciences Naturelles et d'Archéologie de Toulon et du Var, no 26, p. ??.

Decloître, L., 1975a, Thécamoebiens observés dans la zone littorale et supra-littorale au Brusc à Six-Fours-les-Plages: Annales de la Société des Sciences Naturelles et d'Archéologie de Toulon et du Var, no 27, p. 171-176.

Decloître, L., 1975b, Thécamoebiens observés dans différents milieux à Tourtour (Var): Annales de la Société des Sciences Naturelles et d'Archéologie de Toulon et du Var, no 27, p. 177-179.

Decloître, L., 1975c, Thécamoebiens d'un sol cultivé en framboisier: Annales de la Société des Sciences Naturelles et d'Archéologie de Toulon et du Var, no 27, p. 180.

Decloître, L., 1976a, Le genre Euglypha. Compléments à jour au 31 décembre 1974 de la monographie du genre parve en 1962: Archiv für Protistenkunde, v. 118, no 1, 2, p. 18-33.

Decloître, L., 1976b, Le Genre Arcella Ehrenberg. Compléments à jour au 31 décembre 1974 de la monographie du genre parve en 1928: Archiv für Protistenkunde, v. 118, no 1, 2, p. 291-309.

Decloître, L., 1976c, Thécamoebiens observés dans différents milieux dans le départment du Var: Annales de la Société des Sciences Naturelles et d'Archéologie de Toulon et du Var, no 28, p. 174-180.

Decloître, L., 1976d, Thécamoebiens du Gouffre de la Ripelle: Annales de la Société des Sciences Naturelles et d'Archéologie de Toulon et du Var, no 28, p. 180.

Decloître, L., 1976e, Thécamoebiens des sables des bords de mer, Plage de Bonne- 
Grâce à Six-Fours-les-Plages (Var). 2me note: Annales de la Société des Sciences Naturelles et d'Archéologie de Toulon et du Var, no 28, p. 181-183.

Decloître, L., 1976f, Thécamoebiens des îles Galapagos: Ciencia y Naturaleza (Quito), v. 14, p. 11-20.

Decloître, L., 1976g, Thécamoebiens de l'île de Tahiti: Archives Originales du Centre National de la Recherche Scientifique, no 560, p. ??

Decloître, L., 1976h, Le genre Arcella Ehrenberg, complements a jour au 31 decembre 1974 de la monographie du genre parue en 1928: Archiv für Protistenkunde, v. 118, pp. 291-309.

Decloître, L., 1977a, Le genre Cyclopyxis. Compléments à jour au 31 décembre 1974 de la monographie du genre parve en 1929: Archiv für Protistenkunde, v. 119, p. 3153.

Decloître, L., 1977b, Thécamoebiens de quelques rhizosphères dans le Var: Annales de la Société des Sciences Naturelles et d'Archéologie de Toulon et du Var, no 29, p. 164-166.

Decloître, L., 1977c, Thécamoebiens d'une pinède dans le Var: Annales de la Société des Sciences Naturelles et d'Archéologie de Toulon et du Var, no 29, p. 167.

Decloître, L., 1977d, Thécamoebiens des bords de mer, zone littorale et supralittoral, Six-Fours-les-Plages (Var): Annales de la Société des Sciences Naturelles et d'Archéologie de Toulon et du Var, no 29, p. 168-170.

Decloître, L., 1977e, Thécamoebiens observés dans différents milieux dans le département du Var à Tourtour le 22 juin 1976: Annales de la Société des Sciences Naturelles et d'Archéologie de Toulon et du Var, no 29, p. 171-173.

Decloître, L., 1977f, Le genre Nebela. Compléments à jour au 31 décember 1974 de la monographie du genre parve en 1936: Archiv für Protistenkunde, v. 119, no 4, p. 325352.

Decloître, L., 1977g, Faune thécamoebienne de France à jour au 31-XII 1976: Archives Originales du Centre National de la Recherche Scientifique, no 401, p. ??.

Decloître, L., 1977h, Faune thécamoebienne d'Europe par pays et région: Archives Originales du Centre National de la Recherche Scientifique, no 403, p. ??.

Decloître, L., 1977i, Faune thécamoebienne d'Europe: Archives Originales du Centre National de la Recherche Scientifique, no 403, p. ??.

Decloître, L., 1977j, Faune thécamoebienne du monde: Archives Originales du Centre National de la Recherche Scientifique, no 404, p. ?? 
Decloître, L., 1977k, Faune thécamoebienne de la zone supralittorale marine et des milieux saumâtres: Archives Originales du Centre National de la Recherche Scientifique, no 520, p. ??.

Decloître, L., 1977I, Thécamoebiens de Turquie: Archives Originales du Centre National de la Recherche Scientifique, no 561, p. ??.

Decloître, L., 1978a, Le genre Centropyxis I. Compléments à jour au 31 décembre 1974 de la Monographie du genre parve en 1929: Archiv für Protistenkunde, v.120, p.63-85.

Decloître, L., 1978b, Thécamoebiens récoltés sur feuilles de différents végétaux: Annales de la Société des Sciences Naturelles et d'Archéologie de Toulon et du Var, no 30, p. 191-197.

Decloître, L., 1978c, Thécamoebiens observés dans le haut Var: Annales de la Société des Sciences Naturelles et d'Archéologie de Toulon et du Var, no 30, p. 198-201.

Decloître, L., 1978d, Recherches aux plages du Brusc et de Bonne Grâce à Six-Fours: Annales de la Société des Sciences Naturelles et d'Archéologie de Toulon et du Var, no 30, p. 202.

Decloître, L., 1979a, Le genre Centropyxis II. Compléments à jour au 31 décembre 1974 de la Monographie du genre parue en 1929: Archiv für Protistenkunde, v. 121, no 1, 2, p. 162-192.

Decloître, L., 1979b, Mises à jour au 31.12.1978 des mises à jour au 31.12.1974 concernant les genres Arcella, Centropyxis, Cyclopyxis, Euglypha et Nebela: Archiv für Protistenkunde, v. 122, no 3, 4, p. 387-397.

Decloître, L., 1979c, Thécamoebiens récoltés sur feuilles de différents végétaux: Annales de la Société des Sciences Naturelles et d'Archéologie de Toulon et du Var, no 31, p. 148-155.

Decloître, L., 1979d, Thécamoebiens reconnus dans le massif des cèdes du Lubéron: Archives Originales du Centre National de la Recherche Scientifique, no 590, p. ??.

Decloître, L., 1979e, Thécamoebiens observés sur feuilles de plantes exotiques cultivées en France: Annales de la Société des Sciences Naturelles et d'Archéologie de Toulon et du Var, no 31, p. 156-159.

Decloître, L., 1979f, Thécamoebiens observés de la station de Chandourène, près de Digne: Archives Originales du Centre National de la Recherche Scientifique, no 589 , p. ??.

Decloître, L., 1980a, Thécamoebiens observés dans le sol. Pépinières Clément à La 
Crau (Les Meissoniers): Annales de la Société des Sciences Naturelles et d'Archéologie de Toulon et du Var, no 32, p. 168-171.

Decloître, L., 1980b, Thécamoebiens d'humus d'une pinède à Six-Fours: Annales de la Société des Sciences Naturelles et d'Archéologie de Toulon et du Var, no 32, p. 172-173.

Decloître, L., 1980c, Thécamoebiens des sables des bords de mer: Annales de la Société des Sciences Naturelles et d'Archéologie de Toulon et du Var, no 32, p. 174.

Decloître, L., 1980d, Thécamoebiens observés sur feuilles de végétaux à Six-Fours: Annales de la Société des Sciences Naturelles et d'Archéologie de Toulon et du Var, no 32, p. 175.

Decloître, L., 1981a, Le genre Trinema Dujardin, 1841. Révision à jour au 31 XII. 1979: Archiv für Protistenkunde, v. 124, p. 193-218.

Decloitre, L. (1982) The genera Arcella, Centropyxis, Cylopyxis, Nebela and Trinema. Supplements closed 31.12.1981. Archiv für Protistenkunde, 126 393-407.

Decloître, L., 19??, Thécamoebiens observés dans des Rhizosphères du Var: Annales de la Société des Sciences Naturelles et d'Archéologie de Toulon et du Var, no ??, p. ??. date, no, pages unknown.

Decloître, L., 19??, Thécamoebiens observés sur feuilles de différents végétaux de la Région de Six-Fours-les-Plages: Annales de la Société des Sciences Naturelles et d'Archéologie de Toulon et du Var, no ??, p. ??. date, no, pages unknown.

Decloître, L., 19??, Thécamoebiens observés dans un humus de pinède: Annales de la Société des Sciences Naturelles et d'Archéologie de Toulon et du Var, no ??, p. ??. date, no, pages unknown.

Decloître, L., 19??, Thécamoebiens observés dans des humus naturels dans le HautVar: Annales de la Société des Sciences Naturelles et d'Archéologie de Toulon et du Var, no ??, p. ??. date, no, pages unknown.

Decloître, L., 19??, Thécamoebiens dans le sol dans le Haut-Var: Annales de la Société des Sciences Naturelles et d'Archéologie de Toulon et du Var, no ??, p. ??. date, no, pages unknown.

Decloître, L., Cailleux, A., 1980, Sur la repartition taxonomique et geographique des Thécamoebiens et des Angiospermes: Geographie Physique et Quaternaire, v. 34, pp. 351-358. 


\section{DEF-EGB}

Deflandre, G. and Deflandre-Rigaud, M., 1958, Difflugia? marina Bailey, une espèce oubliée, synonyme de Quadrulella symmetrica (Wallich), Rhizopode testacé d'eau douce. Remarques sur la systématique des Nebelidae: Hydrobiologia, v. 12, no 4, p. 299-307.

Deflandre, G., 19??, Eugène Penard (1855-1954). Correspondance et souvenirs. Bibliographie et bilan systématique de son oeuvre: Hydrobiologia, v. 10, p. 20-37??. date, pages

Deflandre, G., 1926, Matériaux pour la faune Rhizopodiques de France. Rhizopodes du Confolentais: Feuille Naturalistes, v. 33, p. 169-170.

Deflandre, G., 1926a. Notes sur quelques Rhizopodes et Héliozoaires du Venezuela: Bulletin de la Société Zoologique de France, v. 51, p. 515-530.

Deflandre, G., 1927, Matériaux pour la faune rhizopodique de France: -III: Bulletin de la Société Zoologique de France, v. 52, p. 496-519.

Deflandre, G., 1928a, Le genre Arcella Ehrenberg. Morphologie-Biologie. Essai phylogénétique et systématiqe: Archiv für Protistenkunde, v. 64, p. 152-287.

Deflandre, G., 1928b, Deux genres nouveaux de Rhizopodes testacés: Annales de Protistologie, p. 37-43.

Deflandre, G., 1928c, A propos du genre "Arcella": Annales de Protistologie, v. 1, p. 198.

Deflandre, G., 1929a, Le genre Centropyxis Stein: Archiv für Protistenkunde, v. 67, p. 322-375.

Deflandre, G., 1929b, Observations sur les mouvements propres, pistes et vitesses de déplacement de quelques Protistes: Annales de Protistologie, v. 2, p. 1-40.

Deflandre, G., 1931, Thécamoebiens nouveaux ou peu connus, I: Annales de Protistologie, v. 3, no 2, 3, p. 81-95, pls. 11-17.

Deflandre, G., 1932, Paraquadrula nov. gen. irregularis (Archer). Conjugaison et enkystement: Comptes rendus hebdomadaires des Séances de la Société de Biologie et de ses filiales et associées (Paris), v. 109, no 15, p. 1346-1347.

Deflandre, G., 1936a, Remarques sur le comportement des pseudopodes chez quelques Thécamoebiens: Annales de Protistologie, v. 5, p. 65-71.

Deflandre, G., 1936b, Etude monographique sue le genre Nebela Leidy (RhizopodaTestacea): Annales de Protistologie, v. 5, p. 201-322, pls. 10-27. 
Deflandre, G., 1937, Adaption stationnelle et notion d'espèce chez les Thécamoebiens in Comptes rendus du LXX Congrès des Sociétés Savantes de Paris et des Départements. Section des Sciences, p. 223-225.

Deflandre, G., 1952, Groupe des Thécamoebiens in Piveteau, J. (ed.), Traité de paléontologie, $v$. I: Les stades inférieurs d'organisation du règne animal. Introduction. Généralités. Protistes. Spongiaires. Coelentérés. Bryozoaires, $p$. 131-132.

Deflandre, G., 1953, Ordres des Testaceolobosa (de Saedeleer,1934), Testaceafilosa (de Saedeleer, 1934), Thalamia (Haeckel, 1862) ou Thécamoebiens (Auct.) (Rhizopoda Testacea) in Grassé, P.-P., (ed.), Traité de Zoologie, v. I, fasc. II, p. 97-148, pl. 1; Masson and Co. (Paris).

Deflandre, G., 1953a, Super-classe des Rhizopodes (Rhizopoda von Siebold, 1845, Sarkodina Hertwig et Lesser, 1874). Généralités in Grassé, P.-P., (ed.), Traité de Zoologie, v. I, fasc. II, p. 3-4; Masson and Co. (Paris).

Deflandre, G., 1954a, Eugène Penard (1855-1954), sa vie et son oeuvre: Journal of Protozoology, v. 1, no 3, p. 187-190.

Deflandre, G., 1954b, Eugène Penard et la protistologie: La Nature (Paris), no 3227, p. 110-111.

Deflandre, G., 1959, Rhizopoda and Actinopoda in Edmondson, W. T. (ed.), (H. B. Ward and G. C. Whipple's) Fresh-water biology, 2nd. edition, p. 232-264; John Wiley and Sons, Inc. (New York).

Delage, Y., Hérouard, E., 1896, Traité de zoologie concrète. v. I: la cellule et les Protozoaires: p. I-XXX, 1-584, pls.1-15; C. Reinwald/Schleicher Bros. (Paris).

Delphy, J., 1936, Sous-règne des Protozoaires in Perrier, R. (ed.), La Faune de la France en tableaux synoptiques illustrés, v. 1A, p. PZ1-PZ95, II-III; Delagrave (Paris).

De Tarr, D. N., 1884, A list of the Rhizopoda found in the vicinity of Albany, N.Y.: Report of the New York State Museum, v. 35, p. 165-167.

Diesing, C. M., 1848, Systematische Uebersicht der Foraminiferen monostegia und Bryozoa anopisthia: Sitzungsberichte der Kaiserlichen Akademie der Wissenschaften, v. 1, p. 494-527.

Dioni, V., 1970, Taxocenos de tecamebianos en cuencas islenas de Parana Medio 1: Acta Zoologica Lilloana, v. 27, p. 201-240.

Doflein, F., 1908, Ueber den Theilungsvorgang bei den Süsswasserthalamophoren: Sitzungsberichte der Gesellschaft für Morphologie und Physiologie in München, v. 23 , no 2 , p. $115-125$. 
Doflein, F., 1916, Studien zur Naturgeschichte der Protozoen. VIII. Pyxidicula operculata: Zoologische Jahrbuch, Abhandlungen, Anatomie, v. 39, p. 585-??.

Doflein, F., Reichenow, E., 1927-1928-1929, Lehrbuch der Protozoenkunde. Eine Darstellung der Naturgeschichte der Protozoen mit besonderer Berücksichtigung der der parasitichen und pathogenen Formen: content: I. Teil - Allgemeine der Protozoen (1927): p. 1-436; II. Teil - Spezielle Naturgeschichte der Protozoen. (1) Mastigophoren und Rhizopoden (1928), p. 437-864; (2) Sporozoa, Ciliata und Suctoria (1929), p. 8651262; 5. Auflage, Gustav Fisher, Jena.

Doflein, F., Reichenow, E., 1949-1952-1953, Lehrbuch der Protozoenkunde. Eine Darstellung der Naturgeschichte der Protozoen mit besonderer Berücksichtigung der der parasitichen und pathogenen Formen: content: I. Teil - Allgemeine der Protozoen (1949): p. 1-436; II. Teil - Spezielle Naturgeschichte der Protozoen: (1) Mastigophoren und Rhizopoden (1952), p. 409-776; (2) Sporozoa und Ciliophora (1953), p. 777-1214; 5. Auflage, Gustav Fisher, Jena.

Dreyer, F., 1889a, Betrachtungen über den Bau der Rhizopodenschalen: Biologisches Zentralblatt, v. 9, p. 333-352.

Dreyer, F., 1889b, Considerations on the structure of rhizopod shells: Annals and Magazine of Natural History, ser. 6, v. 4, no 22, p. 300-319.

Dreyer, F., 1891, Die Principien der Gerüstbildung bei den Rhizopoden, Spongien und Echinodermen. Ein Versuch zur mechaniscen Erklärung organischer Gebilde:

Jenaische Zeitschrift für Naturwissenschaft, v. 26, p. 204-296, pls. 15-19.

Dreyer, F., 1892a, Die Principien der Gerüstbildung bei den Rhizopoden, Spongien und Echinodermen (Fortsetzung): Jenaische Zeitschrift für Naturwissenschaft, v. 26, v. 19 , p. 297-468, pls. 20-29.

Du Plessis, G., 1876, Arcellina marina, gen. et sp. nov., eine neue Rhizopodenform, aus der Familie der Arcellideen: Sitzungsberichte der Physikalisch-medizinischen Societät zu Erlangen, v. 8, p. 100-107.

Du Plessis, G., 1877, Notice sur un Rhizopode nouveau marin voisin des Arcelles et des Amphizonelles: Bulletin de la Société Vaudoise des Sciences Naturelles (Lausanne), v. 15 (1878), p. 1-13. - This is a translation of Du Plessis 1976.

Du Plessis, G., Gouret, G., 1885, Essai sur la faune profonde des lacs de la Suisse: Mémoires couronnés de la Société Helvétique, v. 34, no 2, p. 1-64.

Dubois, G., Dubois, C., 1943, Notes paléontologiques sur le Tonnelet des Tourbières (Amphitrema flavum Archer): Bulletin de la Société Géologique de France, ser. 5, v. 13, p. 21-36.

Dujardin, F., 1835a, Observations nouvelles sur les Céphalopodes microscopiques: Annales de Science Naturelles, Zoologie, ser. 2, v. 3, p. 108-109. 
Dujardin, F., 1835b, Observations nouvelles sur les prétendus Céphalopodes microscopiques: Annales de Science Naturelles, Zoologie, ser. 2, v. 3, p. 312-314.

Dujardin, F., 1835c, Recherches sur les organismes inférieurs: Annales de Science Naturelles, Zoologie, ser. 2, v. 4, p. 343-377, pl. 9-11.

Dujardin, F., 1835d, Observations sur les rhizopodes et les infusoires: Comptes Rendus hebdomadaires des Séances de l'Académie des Sciences de Paris, v. 1, p. 338-340.

Dujardin, F., 1836a, Recherches sur les organismes inférieurs: Annales de Science Naturelles, Zoologie, ser. 2, v. 5, p. 193-206.

Dujardin, F., 1836b, Rhizopodes d'eau douce: Extraits des Procès-verbaux des Séances de la Société Philomatique de Paris, p. 26-28.

Dujardin, F., 1837, Sur une nouvelle espèce de Gromia et sur les Difflugies: Annales des sciences Naturelles, Zoologie, ser. 2, v. 8, p. 310-313, pl. 9.

Dujardin, F., 1838a, Mémoire sur l'organisation des infusoires: Annales des sciences Naturelles, Zoologie, ser. 2, v. 10, p. 230-315.

Dujardin, F., 1838b, Animaux microscopique: Procès-verbaux des Séances de la Société Philomatique de Paris, p. 7.

Dujardin, F., 1840, Mémoires sur une classification des infusoires en rapport avec leur organisation: Comptes Rendus hebdomadaires des Séances de l'Académie des Sciences de Paris, v. 11, no 7, p. 281-286.

Dujardin, F., 1841, Histoire naturelle des Zoophytes. Infusoires, comprenant la physiologie et la classification de ces animaux, et la manière de les étudier à l'aide du microscope: De Roret, collection "Nouvelle suites a Buffon, formant, avec les oeuvres de cet auteur, un cours complet d'Histoire naturelle." (Paris). There are two volumes: a text volume (p. I-XII, and 1-684) and an Atlas of plates (p. 1-14, pls. 1 16,16 bis, 17-22).

Eckert, B. S., Mcgee-Russell, S. M., 1973a, The patterned organization of thick and thin microfilaments in the contracting psuedopod of Difflugia: Journal of Cell Science, v. 13 , no 1 , p. $727-739$.

Eckert, B. S., Mcgee-Russell, S. M., 1974, Shell structure in Diffluqia lobostoma observed by scanning and transmission electon microscopy: Tissue and cell, v. 6, no 2, p. 215-221.

Eckert, B. S., Mcgee-Russell, S. M., 1973b, Scanning electron microscopy of dried and acid tested shells of Difflugia in Arcenaux, C.J. (ed.), Proceedeings of the 31st Annual Meeting of the Electron Microscopy Society of America, New Orleans (Louisiana), p. 448-449. 
Edmonson, C. H., 1906, The Protozoa of lowa. A study of species known to occur in the waters of this State: Davenport Academy of Sciences Proceedings, v. 11, p. 1124, pls. 1-30.

Edmonson, C. H., 1910, A report on the fresh-water Protozoa of Tahiti: Science, new ser., v. 32, p. 349-351.

Edmonson, C. H., 1912, Protozoa of high mountain lakes in Colorado: University of Colorado Studies (University of Colorado, Boulder), ser. A, v. 9, no 2-3, p. 65-74.

Edmonson, C. H., 1918, Amoeboid Protozoa in Ward and Whipple (eds.), Freshwater Biology, p. 210-237, New York and London.

Egborge, A.B.M., 1972, A preliminary checklist of the zooplankton organisms of the River Oshun in the Western State of Nigeria: Nigerian Journal of Science, v. 6, p. 6772. 


\section{EHR}

Ehrenberg, G. C., 1830, Organisation, Systematik und geographisches Verhältniss der Infusionsthierchen: p.1-108, pls. 1-8 : Printed by: Druckerei der Königlichen

Akademie der Wissenschaften (Berlin). - This paper is almost identical to Ehrenberg $1832 \mathrm{a}$ and $1832 \mathrm{~b}$ combined.

Ehrenberg, G. C., 1832a, Die geographische Verbreitung der Infusionsthierchen in Nord-Afrika und West-Asien, beobachtet auf Hemprich und Ehrenberg's Reisen: Königliche Akademie der Wissenschaften zu Berlin, Physikalische Abhandlungen (1829), p. 1-20.

Ehrenberg, G. C., 1832b, Beiträge zur Kenntnis der Organisation der Infusorien und ihrer geographischen Verbreitung, besonders in Sibirien: Königliche Akademie der Wissenschaften zu Berlin, Physikalische Abhandlungen (1830), p. 1-88, pl. 1-8.

Ehrenberg, G. C., 1832c, Zur Erkenntniss der Organisation in der Richtung des kleinsten Raumes. Zweiter Beitrag. Entwinckelung, Lebensdauer und Structur der Magenthiere und Räderthiere, oder sogenannten Infusorien, nebst einer physiologischen Charakteristik beiden Klassen und 412 Arten derselben: Königliche Akademie der Wissenschaften zu Berlin, p. 1-154, pls.1-4. - Except for the title, this paper and Ehrenberg 1832d are identical.

Ehrenberg, G. C., 1832d, Über die Entwickelung und Lebensdauer der Infusionsthiere, nebst ferneren Beiträgen zu einer Vergleichung ihrer organischen Systeme: Königliche Akademie der Wissenschaften zu Berlin, Physikalische Abhandlungen (1831), $p$. 1-154, pl. 1-4.

Ehrenberg, G. C., 1835, Dritter Beitrag zur Erkenntniss grosser Organisation in der Richtung des kleinster Raumes: Abhandlungen der Königliche Akademie der Wissenschaften zu Berlin (1833), p. 149-336, 11 pls.

Ehrenberg, G. C., 1836, Mittheilung einer sehr einfachen Methode zum Fashalten, vergleiche, und Aufbewahren der Fernsten und verganglichsten mikroskopischen Objecte: Königliche Akademie der Wissenschaften zu Berlin, Physikalische Abhandlungen (1835), p. 14-149.

Ehrenberg, G. C., 1837, Zusätze zur Erkenntniss grosser organischer Ausbildung in den kleinsten Thierischen Organismen: Königliche Akademie der Wissenschaften zu Berlin, Physikalische Abhandlungen (1835), p. 151-180, pl. 1

Ehrenberg, G. C., 1838, Die Infusionsthierchen als vollkommene Organismen. Ein Blick in das tiefere organische Leben der Natur:, 2 vols., p. i-xviii, 1-547, pls. 1-64., L. Voss (Leipzig).

Ehrenberg, G. C., 1839a, Die Bildung der europäischen, libyschen und arabischen Kreidefelsen und des Kreidemergels aus mikroskopischen Organismen, dargestellt und 
physiologisch erläutert: p.1-91, Printed by: Königliche Akademie der Wissenschaften zu Berlin (Berlin). - This book is almost identical to Ehrenberg 1840b.

Ehrenberg, G. C., 1839b, Eine vorläufige Übersicht seiner Untersuchungen der Schecken-Corallen oder Polythalamien als Thiere: Bericht über die zur Bekanntmachung geeigneten Verhandlungen der Königliche Preussischen Akademie der Wissenschaften zu Berlin, no 12. p. 196-200.

Ehrenberg, G. C., 1840a, (no title: Loeblich and Tappan (1964) gave it the following one: "Das grössere Infusorienwerke"): Bericht über die zur Bekanntmachung geeigneten Verhandlungen der Königliche Preussischen Akademie der Wissenschaften zu Berlin, v. 5, p. 197-219.

Ehrenberg, G. C., 1840b, Über die Bildung der Kreidefelsen und des Kreidemergels durch unsichtbare Organismen, Abhandlungen der Königliche Akademie der Wissenschaften zu Berlin (1838), p. 59-147, pls. 1-4.

Ehrenberg, G. C., 1843a, Verbreitung und Einfluss des mikroskopischen Lebens in Süd- und Nord-Amerika: Abhandlungen der Königliche Akademie der Wissenschaften zu Berlin (1841), p. 291-446, pls. 1-4.

Ehrenberg, G. C., 1843b, Über die weitere Entwickelung der Verbreitung und des Rinflusses des mikroskopischen Lebens in Afrika: Bericht über die zur Bekanntmachung geeigneten Verhandlungen der Königliche Preussischen Akademie der Wissenschaften zu Berlin, v. 8, p. 133-143.

Ehrenberg, G. C., 1843c, Über den sichtlichen Einfluss der mikroskopischen MeeresOrganismen auf den Baden des Elbbettes bis oberhalb Hamburg: Bericht über die zur Bekanntmachung geeigneten Verhandlungen der Königliche Preussischen Akademie der Wissenschaften zu Berlin, v. 8, p. 160-167.

Ehrenberg, G. C., 1844, Untersuchungen über die kleinsten Lebensformen im Quellenlande des Euphrats und Araxes, so wie über eine an neuen Formen sehr reiche marine Tripelbildung von den Bermuda-Inseln: Bericht über die zur Bekanntmachung geeigneten Verhandlungen der Königliche Preussischen Akademie der Wissenschaften zu Berlin, v. 9, p. 253-275.

Ehrenberg, G. C., 1845a, Vorläufige zweite Mittheilung über die weitere Erkenntniss der Beziehungandes kleinsten organischen Lebens zu der vulkanischen Masser der Erde: Bericht über die zur Bekanntmachung geeigneten Verhandlungen der Königliche Preussischen Akademie der Wissenschaften zu Berlin, p. 133-157.

Ehrenberg, G. C., 1845b, Über das kleinste organische Leben an meheren bisher nicht untersuchten Erdpunkten vor: Bericht über die zur Bekanntmachung geeigneten Verhandlungen der Königliche Preussischen Akademie der Wissenschaften zu Berlin, v. 10, p. 304-322. 
Ehrenberg, G. C., 1845c, Über das kleinste organische Leben an meheren bisher nicht untersuchten Erdpunkten; mikroskopische Lebensformen von Portugal und Spanien, Süd-Afrika, Hinter-Indian, Japan und Kurdistan: Bericht über die zur Bekanntmachung geeigneten Verhandlungen der Königliche Preussischen Akademie der Wissenschaften zu Berlin, v. 10, p. 357-377.

Ehrenberg, G. C., 1846, Mikroskopische Organismen in den vulkanischen Ablagerungen am Laucher-See: Bericht über die zur Bekanntmachung geeigneten Verhandlungen der Königliche Preussischen Akademie der Wissenschaften zu Berlin, v. 11, p. 158-173.

Ehrenberg, G. C., 1848a, Über eigenthümliche auf den Bäumen des Urwaldes in SüdAmerika zahlreich lebende mikroskopische oft kieselschalige Organismen: Bericht über die zur Bekanntmachung geeigneten Verhandlungen der Königliche Preussischen Akademie der Wissenschaften zu Berlin, v. 13, p. 213-220.

Ehrenberg, G. C., 1848b, Über das gewöhnlich in der Atmospäre insichtbar getragene formenreiche Leben: Bericht über die zur Bekanntmachung geeigneten Verhandlungen der Königliche Preussischen Akademie der Wissenschaften zu Berlin, v. 13, p. 325-345, 349-362.

Ehrenberg, G. C., 1848c, Fortgesetzte Beobachtungen über jetzt herrschende atmosphärische mikroskopische Verhältnisse: Bericht über die zur Bekanntmachung geeigneten Verhandlungen der Königliche Preussischen Akademie der Wissenschaften zu Berlin, v. 13, p. 370-381.

Ehrenberg, G. C., 1848d, Über die in der heissen Quelle des Rio-Taenta-Flusses in Afrika, im Innern von Mosambik vorkommenden mikroskopischen Organismen: Bericht über die zur Bekanntmachung geeigneten Verhandlungen der Königliche Preussischen Akademie der Wissenschaften zu Berlin, v. 13, p. 225-227.

Ehrenberg, G. C., 1849a, Passatstaub und Blutregen; ein grosses organisches unsichtbares Wirken und Leben in der Atmosphäre: Königliche Akademie der Wissenschaften zu Berlin, Physikalische Abhandlungen (1847), v. 32, p. 269-460, pls. 1-6. - This article is almost identical to Ehrenberg (1849b).

Ehrenberg, G. C., 1849b, Passatstaub und Blutregen; ein grosses organisches unsichtbares Wirken und Leben in der Atmosphäre: Königliche Akademie der Wissenschaften zu Berlin, p. 1-192, pls. 1-6.

Ehrenberg, G. C., 1849c, Über das mächtigste bis jetzt bekannt gewordene Lager von mikroskopischen reinen kieselschaligen Süsswasserformen am Wasserfal-Flüsse im Oregon: Bericht über die zur Bekanntmachung geeigneten Verhandlungen der Königliche Preussischen Akademie der Wissenschaften zu Berlin, v. 14, p. 76-98.

Ehrenberg, G.C., 1850a, Mikroskopische Bestandtheile der Schwarzerde in Russland: Bericht über die zur Bekanntmachung geeigneten Verhandlungen der Königliche 
Preussischen Akademie der Wissenschaften zu Berlin, p. 268-272.

Ehrenberg, G.C., 1850b, Mikroskopische Bestandtheile der Schwarzerde in Russland: Journal für praktische Chemie, v. 51, p. 172-176.

Ehrenberg, G.C., 1853a, Über die auf den höchsten Gipfeln der Alpen lebenden mikroskopischen Organismen: Bericht über die zur Bekanntmachung geeigneten Verhandlungen der Königliche Preussischen Akademie der Wissenschaften zu Berlin, v. 8, p 315-362.

Ehrenberg, G.C., 1853b, Über einige neue Materialen zür Übersicht des kleinsten Lebens: Bericht über die zur Bekanntmachung geeigneten Verhandlungen der Königliche Preussischen Akademie der Wissenschaften zu Berlin, v. 18, p. 505533.

Ehrenberg, G.C., 1853c, Über das jetzige mikroskopische Süsswasser der GalapagosInseln: Bericht über die zur Bekanntmachung geeigneten Verhandlungen der Königliche Preussischen Akademie der Wissenschaften zu Berlin, v. 18, p. 178179.

Ehrenberg, G.C., 1853d, Über das jetzige mikroskopische Leben als Flusstrübung und Humusland in Florida: Bericht über die zur Bekanntmachung geeigneten Verhandlungen der Königliche Preussischen Akademie der Wissenschaften zu Berlin, v. 18, p. 252-271.

Ehrenberg, G.C., 1854a, Mikrogeologie. Das Erden und Felsen schaffende Wirken der unsichtbar kleinen selbständigen Lebens auf der Erde: v. 1, p. i-xxviii, 1-374, L. Voss (Leipzig).

Ehrenberg, G.C., 1854b, Zur Mikrogeologie von C. G. Ehrenberg: p. 1-31, pls. 1-40, L. Voss (Leipzig).

Ehrenberg, G.C., 1854c, Beitrag zur Kenntniss der Natur und Enstehung des Grünsandes als Zeuge reichen organischen Lebens selbst im unteren VebergangsGebirge: Bericht über die zur Bekanntmachung geeigneten Verhandlungen der Königliche Preussischen Akademie der Wissenschaften zu Berlin, v. 19, p. 374377.

Ehrenberg, G.C., 1854d, Weitere Mittheilungen über die Natur und Entstehung des Grüundsandes: Bericht über die zur Bekanntmachung geeigneten Verhandlungen der Königliche Preussischen Akademie der Wissenschaften zu Berlin, v.19, p. 384-410.

Ehrenberg, G.C., 1856a, Mikrogeologie. Das Erden und Felsen schaffende Wirken der unsichtbar kleinen selbständingen Lebens auf der Erde. Fortsetzung: p. 1-88, L.Voss (Leipzig).

Ehrenberg, G.C., 1856B, Über das mikroskopische Leben des centralen Landflächen 
Mittel-Afrika's: Monatsberichte der Königlichen Preussische Akademie der Wissenschaften zu Berlin, p. 323-338, 1 pl.

Ehrenberg, G.C., 1856c, - Title and other data unknown - Monatsberichte der Königlichen Preussische Akademie der Wissenschaften zu Berlin (fide: Ehrenberg 1872b, p. 236).

Ehrenberg, G.C., 1856d, Über den Grünsand seine Erläuterung des organischen Lebens: Königliche Akademie der Wissenschaften zu Berlin, Physikalische Abhandlungen (1855), p. 85-176, pls. 1-7.

Ehrenberg, G. C., 1859a, Beitrag zur Bestimmung des stationären mikroskopischen Lebens in bis 20,000 Fuss Alpenhöhle: Königliche Akademie der Wissenschaften zu Berlin, Physikalische Abhandlungen (1858), p. 429-456, 3 pls.

Ehrenberg, G. C., 1859b, Über die mit dem Proteus anguinus (Hypochthon Laurenti) zusammenlebenden mikroskopischen Thierformen in den Bassins der Magdalenengrotte in Krain: Monatsberichte der Königlichen Preussische Akademie der Wissenschaften zu Berlin, p. 758-775.

Ehrenberg, G. C., 1860, Über zwei neue Oberflächen-Erden aus Central-Afrika: Monatsberichte der Königlichen Preussische Akademie der Wissenschaften zu Berlin, p. 151-157.

Ehrenberg, G. C., 1861a, Über die Tiefgrund-Verhältnisse des Oceans am Eingange der Davis-Strasse und bei Isländ: Monatsberichte der Königlichen Preussische Akademie der Wissenschaften zu Berlin, p. 275-315.

Ehrenberg, G. C., 1861b, Über die neveren die Japanische Glaspflanze als Spongia betreffenden Ansichten, und Erläuterungen der Synonyme zu Hrn. Bowerbank's Spongolithen: Monatsberichte der Königlichen Preussische Akademie der Wissenschaften zu Berlin, p. 448-452.

Ehrenberg, G. C., 1862a, Über das mikroskopiosche Leben auf der Insel St.Paul im Süd-Ocean: Monatsberichte der Königlichen Preussische Akademie der Wissenschaften zu Berlin (1861), p. 1085-1102.

Ehrenberg, G. C., 1862b, On the microscopic life of the Island of St.Paul, in the Southern Ocean: Annals and Magazine of Natural History, ser. 3, v. 9, no 53, p. 353366.

Ehrenberg, G. C., 1862c, Über die mikroskopischen Lebensformen als Nahrung des Höhlen-Salamanders: Monatsberichte der Königlichen Preussische Akademie der Wissenschaften zu Berlin, p. 579-598.

Ehrenberg, G. C., 1869a, Über mächtige Gebirgs-Schichten vorherrschend aus mikroskopischen Bacillarien unter und bei der Stadt Mexico: bibliographic data unknown, p. 1-66, pls. 1-2. - This is probably the book version of the article by 
Ehrenberg 1870.

Ehrenberg, G. C., 1869b, Die mikroskopische Lebensverhältnisse auf der Oberfläche der Insel Spitsbergen: Monatsberichte der Königlichen Preussische Akademie der Wissenschaften zu Berlin, p. 257-262.

Ehrenberg, G. C., 1870, Über mächtige Gebirgs-Schichten vorherrschend aus mikroskopischen Bacillarien unter und bei der Stadt Mexico: Königliche Akademie der Wissenschaften zu Berlin, Physikalische Abhandlungen (1869), v. 54 (?), p. 1-66, pls. 1-2.

Ehrenberg, G. C., 1871, Übersicht der seit 1847 fortgesetzten Untersuchungen über das von der Atmosphäre unsichtbar getragene reiche organische Leben: Königliche Akademie der Wissenschaften zu Berlin, p. 1-150, pls. 1-2. - This is the book version of the article by Ehrenberg 1872a.

Ehrenberg, G. C., 1872a, Übersicht der seit 1847 fortgesetzten Untersuchungen über das von der Atmosphäre unsichtbar getragene reiche organische Leben: Königliche Akademie der Wissenschaften zu Berlin, Physikalische Abhandlungen (1871), p. 1-150, pls. 1-2.

Ehrenberg, G. C., 1872b, Nacthrag zur Übersicht der organischen Atmosphärilien: Königliche Akademie der Wissenshaften zu Berlin, Physikalische Abhandlungen (1871), p. 233-275, pl. 3.

Ehrenberg, G. C., 1872c, Mikrogeologische Studien als Zusammenfassung seiner Beobachtungen des kleinsten Lebens der Meeres-Tiefgründe aller Zonen und dessen geologischen Einfluss: Monatsberichte der Königlichen Preussische Akademie der Wissenschaften zu Berlin, p. 265-322.

Ehrenberg, G. C., 1874, Das unsichtbar wirkende Leben der Nordpolarzone am Lande und in den Meerestiefgründe bei 300 mal verstärkter Sehkraft, nach Materialen der Germania erläutert: in: Hartlaub, G. \& Lindeman, M. (eds.): Die zweite deutsche Nordpolarfahrt in den Jahren 1869 und 1870 unter Führung des Kapitän Karl Koldewey: v. 2, p. 437-467. 


\section{ELL-GOL}

Ellinger, T., 1914, Protozoa: Meddelelser om Grnland: v. 23, part 3, p. 743-951.

Ellison, R.L., 1972, Ammobaculites, foraminiferal proprietor of Chesapeake Bay estuaries: Memoir of the Geological Society of America, v. 133, Environmental framework of coastal plain estuaries, pp. 247-262.

Ellison, R.L, 1995, Paleolimnological analysis of Ullswater using testate amoebae: Journal of Paleolimnology, v. 13, pp. 51-63.

Ellison, R.L., Ogden, C.G. (1987) A guide to the study and identification of fossil testate amoebae in Quaternary lake sediments. International Review of Hydrobiology, 72 639-652.

Elpatiewsky, W., 1907, Zur Fortpflanzung von Arcella vulgaris Ehrbg.: Archiv für Protistenkunde, v. 10, no 2, 3, p. 441-466, pls. 21-22.

Engelmann, T. W., 1869a, Beitrage zur Physiologie des Protoplasma: Archiv gesamte Physiologie, v. 2, p. 307-322.

Engelmann, T. W., 1869b, Sur le développement périodique de gaz dans le protoplasme des Arcelles vivantes: Nederlandsche Archieven Natuurkunde, v. 4, p. 427-430, pl. 4.

Engelmann, T. W., 1869c, Sur l'irritation électrique des Amibes et des Arcelles: Nederlandsche Archieven Natuurkunde, v. 4, p. 431-442.

Engelmann, T. W., 1870, title???: Nederlandsche Archieven Natuurkunde, v. 5, p. $29-42$.

Engelmann, T. W., 1878, Über Gastenwicklung im Protoplasma lebender Protozoen: Zoologischer Anzeiger, v. 1, p. 152-153.

Engelmann, T. W., 1969d, title???: Nederlandsche Archieven Natuurkunde, v. 4, p. 501-508.

Entz, G., 1877a, A szamosfalvi sóstóban élö gyÖklbúakról. (Rhizopoda).:

Természetrajzi Füzetek (Naturhistorische Hefte, herausgegeben vom Ungarischen National-Museum) (Budapest), v. 1, no 3, p. 154-168, pls. 9, 10. -This paper was translated in german (Entz, 1877b).

Entz, G., 1877b, Ueber die Rhizopoden des Salzteiches zu Szamosfalva: Természetrajzi Füzetek (Naturhistorische Hefte, herausgegeben vom Ungarischen National-Museum) (Budapest), v. 1, no 4, p. 185-199.

Entz, G., 1878a, On the Rhizopoda of the salt lake of Szamosfalva: Annals and Magazine of Natural History, ser. 5, v. 1, no 5, p. 423. 
Entz, G., 1878b, Zur Gasentiwicklung im Protoplasma lebender Protozoen: Zoologischer Anzeiger, v. 1, p. 248-249.

Entz, G., 1882, Das konsortialverhaltnis von Algen und Tieren: Biologische Zentralblatt, v. 2, p. 451-464.

Entz, G., 1888, Tanulmnyok a véglények KÖrebÖl. 1. Rész. A véglények ismeretének fejlödése. TÖrténeti és kritikari tpillants. Studien über Protisten. I. Theil: Entwickelung der Kenntniss der Protisten; ein historisch-kritischer Überblick.: p. i-xv, 1-464; Kiajda a Kir. Majyar Természettudomnyi Trsulat Verlag der Königlichen Ungarischen Naturwissenschaftlichen Gesellschaft (Budapest).

Entz, G., 1897a, Einleitung und allemeine Betrachtungen über die Fauna des Balatonsees in Resultat wissenschaftliche Erforschung des Balatonsees, v. 2, part 1, p. I-XXXIX; (Wien).

Entz, G., 1897a, Einleitung und allemeine Betrachtungen über die Fauna des Balatonsees in Resultat wissenschaftliche Erforschung des Balatonsees, v. 2, part 1, p. I-XXXIX; (Wien).

Entz, G., 1897b, Protozoen aus Neu-Guinea: Matematikai Termeszettudomanyi Ertesito, v. 15, p. 170-184.

Entz, G., 1899, Protozoen aus Neu-Guinea: Math. Naturwissenschaftl. Ber Ungarn, v. 15, p. 181-195.? Ersatz der Fortpflanzung von Amoeba proteus durch fortgesetze regenerationen: Zoologische Jarbucher Abteilung für Allgemeine Zoologie und Physiologie der Tier, v. 45, p. ??.

Erskian, M.G., Lipps, J.H., 1997, Distribution of foraminifera in the Russian River estuary, northern California: Micropaleontology, v. 23, pp. 453-469

Ertl, M., 1954: Kritische Bemerkungen über Difflugia leidyi Wailes: Biologia (Bratislava), v. 2, p. 423-429.

Ertl, M., 1955, Rhizopoda Raseliniska Bor (Orava): Prace II Skcie, Slovenskey, Akademie Vied Svazökk i-z, 12, p. (?)

Ertl, M., 1965: Zur Kenntnis der Testaceenfauna der slowakischen Reisfelder: Hydrobiologia, v. 26, p. 13-20.

Evans, W., 1907, Our present knowledge of the fauna of the Forth area: Proceedings of the Royal Physical Society of Edinburgh, v. 17, no 1, p. 1-64d.

Eyferth, B., 1878, Die einfachsten Lebensformen. Systematische Naturgeschichte der mikroskopischen Süsswasserbewohner: p. (?) (Braunschweig).

Eyferth, B., 1885, Die einfachsten Lebensformen des Thier- und pflanzenreiches. Naturgeschichte der mikroskopischen Süsswasserbewohner 2nd edition: p. (?) 
(Braunschweig).

Eyferth, B., Schoenichen, W., Kalberlah, A., 1900, Die einfachsten Lebensformen des Thier- und pflanzenreiches. Naturgeschichte der mikroskopischen Süsswasserbewohner 2nd edition: p. (?) (Braunschweig), p.???

Fabre-Domergue, P., 1884, Note sur les Rhizopods et les Infusoires des eaux de Toulouse, leur récolte et leur préparation: Société d'Histoire naturelle de Toulouse, Bulletin, no 3, p. 152-188.

Fauré-Fremiet, E., 1905a, Sur l'organisation du Cochlcopium pellucidum (Hertwig et Lesser): Compte rendu de la Société de Biologie et de ses filiales, v. 58, p. 497499.

Fauré-Fremiet, E., 1905b, Sur une sécrétion interne chez Cochlicopium pellucidum: Compte rendu de la Société de Biologie et ses filiales, v. 58, p. 905-907.

Fermor, X., 1913, Einige neue Befunde aus der Entwicklungsgeschichte von Arcella vulgaris (EHRBG.): Archiv für Protistenkunde, v. 31, p. 39-46, pl. 4.

Fielde, A. M., 1887a, Chinese Rhizopods: Proceedings of the Natural Sciences of Philadelphia, for year 1887, p. 31.

Fielde, A. M., 1887b, Notes on fresh-water rhizopods of Swatow, China: Proceedings of the Natural Sciences of Philadelphia, for year 1887, p. 122-123.

Flossner, D., Kasprzak, P., Mothes, G., Ronnenberger, D., Schönborn, W., 1985: title (?), in: Casper, S. J., Lake Strechlin. A temperate oligotrophic Lake, p. 213-218; The Hague.

Foissner, W., 1979, Ökologie und Systematik der edaphischen Protozoen in den Hohen Tavern (Österreich): UNESCO-Programm der Mensch und die Biosphäre (UNESCO-Man and the Biosphere Programme), Deutsches Nationalkomitee, MABMitteilungen, v. 4, p. 40-65.

Foissner, W., 1981a, Die Gemeinschaftsstruktur der Ciliatenzönose in alpinen Böden (Hohe Tavern, Österreich) und Grundlagen für eine Synökologie der terricolen Ciliaten (Protozoa, Ciliophora) in Bödenbiologische Untersuchungen in den Hohen Tavern 1974-1978, p. 7-52, (Innsbruck).

Foissner, W., 1981a, Terrestrial Protozoa as bioindicators: investigaations at a graded ski trail at the Schlossalm near Bad Hofgastein (Austria) in Dryl, S., Kazubski, S. L. And Ploszaj, J. (eds.): Progress in Protozoology. Abstracts of papers submitted to VI International Congress of Protozoology, Warszawa, Poland, July 5-11, 1981, p. 96, (Warszawa).

Foissner, W., 1981b, Die Gemeinschaftsstruktur der Ciliatenzönose in alpinen Böden (Hohe Tavern, Österreich) und Grundlagen für eine Synökologie der terricolen Ciliaten 
(Protozoa, Ciliophora) in Bödenbiologische Untersuchungen in den Hohen Tavern 1974-1978, p. 7-52, (Innsbruck).

Foissner, W., 1994, High numbers of testate amoebae (protozoa) in the benthon of clean, acidified mountain streams: Limnologica, v. 24, pp. 323-331

Foissner, W., Adams, H., 1980, Abundanz, Vertikalverteilung und Artenzahl der terrestrischen Ciliaten und Testaceen einer Almweide und einer Schipiste auf der Schlossalm bei Bad Hofgastein (Österreich): Zoologischer Anzeiger, v. 205, no 3, 4, p. $181-178$.

Foissner, W., Adams, H., 1981b, Die Gemeinschaftsstruktur und Produktion der terricolen Testaceen (Protozoa, Rhizopoda) in einigen Böden der Österreichischen Zentralalpen (Hohe Tavern, Glocknergebiet) in Bödenbiologische Untersuchungen in den Hohen Tavern 1974-1978, p. 53-78.

Foissner, W., Korganova, G.A. 1995, Redescription of three testate amoebae (Protozoa, Rhizopoda) from a Caucasian soil: Centropyxis plagiostoma Bonnet \& Thomas, Cyclopyxis kahli (Deflandre) and C. intermedia Kufferath: Archiv für Protistenkunde, v. 146, pp. 13-28

Forbes, S. A., 1881, Rhizopods as food for young fishes: Academy of Natural Sciences of Philadelphia Proceedings, v. 4, p. 9-10.

Forel, F.A.C., 1885, La faune profonde des Lacs Suisses: Neue Denkschriften des Allgemein Schweizerische Gesellschaft gesamten Naturwissenschaft, v. 19, (Abh. II), p. 1-234.

Forel, F.A.C., 1886, Le Lac Leman. Precis Scientifique 2 me ed., Neuchatel. journal, p. (?)

Forel, F.A.C., 1887, Faune e Flore del lag di Genevra: Bolletino Scientifico, v. 3, p. 86-92.

Forel, F.A.C., 1902, Le Léman. Monographie limnologique: 3 volumes, v. 3, pt.1, (Lausanne), p. (???)

Francé, R, 1897, Protozoen in Resultaten der wissenschaftliche Erforschung des Balatonsees, v. 2, pt. 1, p. 1-64, (Wien)

Francé, R. H., 1913, Das Edaphon, Untersuchungen zur Oekologie der bodenbewohnenden Mikroorganismen: Arbeiten aus dem Biologischen Institut München, no 2, p. 1-99.

Freeman, C. J. Jr., 1974a, Shell formation, cell division and longevity in Lesquereusia spirlis (Ehrenberg) Penard and Pontiqulasia vas (Leidy) Schouteden. Ph.D. thesis, University of South Carolina, p. I-IV, 1-66, pls. 1-2. 
Freeman, C. J. Jr., 1974b, Shell formation, cell division and longevity in Lesquereusia spirlis (Ehrenberg) Penard and Pontiqulasia vas (Leidy) Schouteden. Dissertation Abstracts International, Section B, v. 35, no 4, p. 1507-B.

Frenguelli, G., 1933, Tecamebiani e Diatomee nel Miocene del Neuquen (Patagonia Settentrionale): Bollettino della Società Geologica Italiana, v. 52, p. 33-43, pl. 5.

Frenguelli, J., Cordini, I. R., 1937, La Diatomita de Quilino; Rev. Mus.LaPlata, Sección Geologia, v. 1, p. 67-116.

Frenzel, H.J., 1976, Die Besiedlung des belebten Schlammes in Abhaengigkeit von Temperatur und Nitrifikation: Z.-Wasser-Abwasser-Forsch., v. 9, pp. 71-75

Frenzel, J., 1891, Untersuchungen über die mikroskopische fauna Argentiniens, Vorlaufiger Bericht: Archiv für Mikroskopishe Anatomie, v. 38, p. 1-24.

Frenzel, J., 1892 or 1897, Untersuchungen über die mikroskopische Faune Argentiniens. I. Theil: Die Protozoen. IV. Abtheilung: Bibliotheca Zoologica, v. 4, Heft 12, p. (?)

Fresenius, G., 1857, Beiträge zur Kenntniss Mikroskopischer Organismen: Abhandlungen, Senckenberg. Naturforsch. Gesellsch. v. 2, p. 211-242, pls. 10-12.

Fric, A., Vvre, J., 1897, Untersuchungen über die Fauna der gewässer Böhmens. III.: Archiv für Naturwissenschaftliche Landes durchforschung von Böhmen, v. 10, no 3, p. 1-74, 1 pl.

Früh, J., Schröter, C., 1904, Die Moore der Schweiz mit Berücksichtigung der gesamten Moorfrage in A. Francke (Herausgegeben durch die Stiftung von Wartensee), Beiträge zur Geologie der Schweiz, herausgegeben von der Geologischen Kommission der Schweizerischen naturforschenden Gesellschaft. Geotechnische Series, 3. Lieferung, p. I-XVIII, 1-751, (IX)-XV, pl. 1-5.

Fuhrmann, O., 1897, Recherches sur I faune des lacs alpins du Tessin: Revae suisse de Zoologie, v. 4, p. 489-543.

Fuhrmann, O., 1900, Le plankton du lac de Neuchâtel: Bulletin de la Société Neuchâtel, v. 28, p. 86-99.

Gabriel, B., 1874, Über die Zeugungs- und Entwickelungscyclus von Troglodytes: Tageblatt: deutsch Naturfosch. (???), v.47, p. 210.

Gabriel, B., 1876, Untersuchungen über Morphologie, Zeugung und Entwickelung der Protozoen: Gegenbaurs Morphologisches Jahrbuch, v. 1, no 4, p. 535-572, pl. 20.

Gagliardi, J., 1871, Rhizopods in London: Quarterly Journal of microscopical Science, new ser., v. 11, p. 80-85.

Gams, H., 1927, Die Geschichte der Lunzer See, Moore, Walder: International Revue 
der gesamten Hydrobiologie und Hydrographie, v. 18, p. 47-81.

Garbini, A., 1898a, Un pugillo di plancton del lago di Como: Atti del Reale Istituto Veneto di Scienze, Lettere ed Arti, ser. 7, v. 9 (1897-98), p. 668-679.

Garbini, A., 1898b, Due nuovi Rizopodi limnetici (Difflugia ciclotellina - Heterophrys pavesii): Geologischer Anzeiger, v. 21, no 575, p. 667-670.

Gassowsky, G.N., 1936, Nove Rhizopoda iz ozer Konchezerskoi grupp (v Karelii) [French summary: Quelques Rhizopodes nouveaux des lacs du group de Kontchesero (en Karélie)]: Trud Borodinskoi Biologicheskoi Stantsii/Berichte der Biologischen Borodin Station, v. 8, no 2, p. 101-121.

Gaumont, R., 1972, Rhizopodes in Encyclopedia Universalis, (France), v. 14, p. 246-249.

Gauthier-Liévre, L., Thomas, R., 1954, Les genres Nebela, Paraquadrula et Pseudonebela (Rhizopodes testacés) en Afrique: Bulletin de la Société d'Histoire Naturelle de l'Afrique du Nord, v. 44 (1953), no 7-8, p. 324-366.

Gauthier-Liévre, L., Thomas, R., 1958, Les genres Diffluqia, Pentagonia, Maghrebia et Hooqenraadia (Rhizopodes testacés) en Afrique: Archiv für Protistenkunde, v. 103, no 1-2, p. 241-370, pl. 8-14.

Gauthier-Liévre, L., Thomas, R., 1960, Le genre Cucurbitella Penard: Archiv für protistenkunde, v. 104, no 4, p. 569-602, pl. 39-43.

Geel, E. van, 1976, A paleontological study of Holocene peat bog sections based on the analysis of pollen, spores and micro- and microscopic remains of fungi, algae, cormophytes and animals: p. 1-75; Hugo de Vries Laboratorium, Universiteit van Amsterdam (Amsterdam).

Gegenbaur, C., 1870, Grundzüge der verleichenden Anatomie. 2. Aufl.: p. (?), eds. (?), (Leipzig).

Gegenbaur, C., 1874, Manuel d'anatomie comparée. Traduit par Carl Vogt: p. (?), eds. (?), (Paris).

Gegenbaur, C., 1878, Grundzüge der verleichenden Anatomie. 8. Aufl.: p. (?), eds. (?), , (Leipzig).

Geitler, L., 1927, Bemerkungen zu Paulinella chromatophora: Zoologischer Anzeiger, v. 73, p. 333-(?).

Gel'tser, Yu. G., 1972, Pochvenne rakovinne kornenozhki (Soil shelled rhizopods) in Problem pochvennoi zoologii. Material IV Vsesoyuznogo soveshchaniya, p. 35, (Moskva).

Gel'tser, Yu. G., 1980, Metod izucheniya pochvennkh prosteishikh (Methods of 
studying soil protozoa) in Nikolyuk, V.F. (chief ed.), Gelt'ser, Yu.G., Korganova, G.A and Mavlyanova, M.I. (eds.): Pochvenne prosteishie (The soil Protozoa): Nauka, series Protozoologiya, no 5, p. 154-165, 1 unnumbered plate, (Leningrad).

Gel'tser, Yu. G., Ibadov, R. R., Mordkovich, G. D., 1980, Pochvenne prosteishie kak komponent biogeotsenoza (Soil protozoa as components of the biogeocoenosis) in Nikolyuk, V.F. (chief ed.), Gelt'ser, Yu.G., Korganova, G.A and Mavlyanova, M.I. (eds.): Pochvenne prosteishie (The soil Protozoa): Nauka, series Protozoologiya, no 5, p. 21-35 (Leningrad).

Gel'tser, Yu. G., Korganova, G.A., 1973, Soil Protozoa as a component of biogeocoenosis in De Puytorac, P., Grain, J. (eds.): Progress in Protozoology. Abstracts of papers read at the Fourth International Congress on Protozoology, Clermont-Ferrand, Sept. 1973, p. 51 (Clermont-Ferrand).

Gel'tser, Yu. G., Korganova, G.A., 1975, Rakovinne kornrnozhki (Testacida) v pochvakh SSSR (Shelled rhizopods (Testacida) in the soils of the USSR) in Problem sel'skokhozyaistvennoi nauki v Moskovskom universitete, p.325-343; publisher??. (Moskva).

Gel'tser, Yu. G., Korganova, G.A., 1976, Pochvoobitayushchie rakovinne kornenozhki (Protozoa, Testacida) i ikh indikatsionnooe znachenie (Soil Testacea as bioindicators) in Problem i metod biologicheskoi diagnostiki i indikatsii pochv (Problems and methods of soil biological diagnosis and indication): Nauka, eds.(?), p. 116-140 (Moskva).

Gel'tser, Yu. G., Korganova, G.A., 1980, Adaptasiya prosteishikh k zhizni vpochve i ikh indikatsionnoe znachenie (Adaptation of Protozoa to soil conditions and their role as indicators) in Nikolyuk, V.F. (chief ed.), Gelt'ser, Yu.G., Korganova, G.A and Mavlyanova, M.I. (eds.): Pochvenne prosteishie (The soil Protozoa): Nauka, series Protozoologiya, no 5, p. 36-51, 2 unnumbered plates, (Leningrad).

Gel'tser, Yu., Korganova, G.A., Yakovlev, A. S. And Alekseev, D. A., 1980, Rakovinne kornenozhki (Testacida) pochv (Soil Tetsacea): Nikolyuk, V.F. (chief ed.), Gelt'ser, Yu.G., Korganova, G.A and Mavlyanova, M.I. (eds.): Pochvenne prosteishie (The soil Protozoa): Nauka, series Protozoologiya, no 5, p. 108-142, 1 unnumbered plate (Leningrad).

Gel'tser, Yu.G., Korganova, G.A., Alekseev, D.A., 1978, (The number, distribution and species composition of Protozoan in some soils of the podzolic zone), in ????

Gel'tser, Yu.G., Korganova, G.A., Alekseev, D.A., 1979, Rakovinne amëb (Testacida, Protozoa) v pochvakh (Shelled amoebae (Testacea, Protozoa) in soils) in Dobrovol'skii, G.V. (ed.): Genezis i ékologiya pochv Tsentral'no-Lesnogo Gosudarstvennogo Zapovednika (Genesis and ecology of the soils of the Central Forest State Park): Nauka, p. 197-229, (Moskva). 
Genin, M., Lagarde, E., 1968, Relations entr les teneurs en azote protéique et l'état physiologique du Rhizopode Gromia oviformis Dujardin: Comptes Rendus de l'Academie des Sciences de Paris, ser. D, v. 266, p. 364-366.

Giard A., 1900, Sur un protozoaires nouveau de la famille des Gromidae (Amoebogromia cinnabarina Gd.): Comptes Rendus de la Société de Biologie de Paris, v. 52, p. 377-378.

Gillies, C. D., 1918a, The spine mode of Centropyxis aculeata Stein: Journal and Proceedings of the Royal Society of New South Wales, v. 52, article 2, p. 166-174.

Gillies, C. D., 1918b, On the seasonal distribution of some Queensland species of Arcella Ehrenberg: Proceedings of the Linnean Societry of New South Wales, v. 43, no 170, p. 237-246.

Gilyarov, M. S., 1955, Pochvenne rakovinn ameb (Testacea) i ikh znachenie dlya diagnostiki bolotnkh pochv (Soil shelled amoebae (Testacea) and their significance for the diagnosis of swampy soils): Pochvovedenie, no 10, p. 61-65.

GL, D., 1961, Das Leben der Tisza. X. Die Rizhopodenfauna der auf ungarischen Boden fliessenden oberen Strecke der Tisza im Jahre 1959-1960: Acta Biologica, v. 7, p. 77-83.

GL, D., 1969, Zooplanktonuntersuchungen im Östlichen-Hauptkanal: Acta Biologica, new ser., v. 15, p. 93-100, 1 pl.

Godeanu, S., 1972, Especes nouvelles de thécamoebiens (Protozoa, Rhizopoda, Arcellinida): Revue Roumaine de Biologie (sér. zoologique), v. 17, p. 227-236.

Godeanu, S., 1975, Protozoa, Testacea in lonescu, M. (ed.), Fauna (bonei Porile, de Fier) in Milcu, .,(Coord.): Seria Monografica - Grupul de Cercetari Complexe " Porile de Fier: Editura Academiei Republicii Socialiste Romania (Bucureti).

Godeanu, S., 1977, Ökologische Untersuchungen an den Testaceen in de Gewässern des eingedeichten Überschwemmungsgebietes Obretin (Donaudelta): Archiv für Hydrobiologie, Supplementband, v. 52, p. 72-105.

Godeanu, S., Godeanu, M., Ionescu, V., 1973, Variaa anualá a planctonolui dir incintá indiquita Obrein (Delta Dunárii): Peace (?), v. 3, p. 211-260. [what should be bold?]

Godet, P. 1900, Les Protozoaires neuchâtelois: Bulletin de la Société Neuchâteloise des Sciences Naturelles (?), v. 28, p. 61-79.

Goette, A., 1902, Lehruch der Zoologie, ???journal etc.

Goette, A., 1916, Über den Lebenscyclus von Difflugia lobostoma: Archiv für Protistenkunde, v. 37, no 2, p. 93-138, pls. 7-9.

Goldschmidt, R., 1904, Die Chromiden der Protozoen: Archiv für Protistenkunde, v. 
5, no 1 , p. $126-144$

Golemansky, V., 1966, Playfairina valkanovi nov. sp.- une nouvelle (sic) rhizopode d'eau douce (Rhizopoda, Testacea): Doklad Bolgarskoi Akademii nauk/ Comptes rendus de l"Académie bulgare des Sciences, v. 19, no 1, p. 57-59.

Golemansky, V., 1966, Playfairina valkanovi nov. sp.- une nouvelle rhizopode d'eau douce (Rhizopoda, Testacea): Doklad Bolgarskoi Akademii Nauk/ Comptes rendus de l'Académie bulgare des Sciences, v. 19, no 1, p. 57-59.

Golemansky, V., 1967a, Tecamoebiens muscicolas (Rhizopoda, Testacea) de México: Revista de la Sociedad Mexicana de Historia natural, v. 18, p. 73-77.

Golemansky, V., 1967b, Etudes de la faune Rhizopodes (Sarcodina, Rhizopoda) des mousses epiphytes et terricoles en Bulgarie: Izvestiya na Zoologocheskiya Institut (Sophia), v. 24, p. 103-119.

Golemansky, V., 1967c, Materiaux sur la systematique et l'ecologie des thecamoebiens (Protozoa, Rhizopoda) du Lac d'Ohrid: Section des Sciences Naturelles de I'Universite de Skopje, Station Hydrobiologique- Ohrid, v. 14, no 4, p. $1-26$.

Golemansky, V., 1967d, Etude sur les especes et l'ecologie des Rhizopodes (Rhizopoda, Testacea) des Hautes Tourbieres de la Montagne Vitocha: Annuaire de I'Universite de Sophia, Faculte de Biologie, v. 59, p. 17-36.

Golemansky, V., 1968a, Materiaux (sic) sur la faune thecamoebienne (sic) (Rhizopoda, Testacea) de Cuba: Acta Protozoologica, v. 6, fasc. 28, p. 335-340.

Golemansky, V., 1968b, Nouvelle contribution a la connaissance des Thécamoebiens (Rhizopoda, Testacea) en Yougoslavie: Académie Bulgare des Sciences, Bulletin de I'Institut de Zoologie (Sofia), v. 27, p. 57-63.

Golemansky, V., 1968c, On the composition and distribution of the Rhizopod fauna of Upper Smolian Lakes: Académie Bulgare des Sciences, Bulletin de l'Institut de Zoologie (Sofia), v. 27, p. 171-187.

Golemansky, V., 1970a, A list of Testacea (Protozoa, Rhizopoda) from the Duszatyskie Lakes in Poland: Fragmenta Faunistica (Warszawa), v. 16, no 3, p. 21-25.

Golemansky, V., 1970b, Rhizopodes nouveaux du psammon littoral de la mer Noire: Protistologica, v. 6, p. 365-371.

Golemansky, V., 1970c, Contribution a la connaissance des Thecamoebiens (Rhizopoda, Testacea) des eaux souterraines littorales du Golf de Gdansk (Pologne): Academie Bulgare des Sciences, Bulletin de I'Institut de Zoologie (Sofia), v. 31, p. 77-87. 
Golemansky, V., 1970d, Psammonobiotus communis Golemansky, 2968 (Rhizopod, Testacea) une relicte marine du Lac d'Ohrid: Académie Bulgare des Sciences, Bulletin de l'Institut de Zoologie (Sofia), v. 32, p. 63-67.

Golemansky, V., 1970e, Thecamoebiens (Rhizopoda, Testacea) des eaux souterraines littorales de quelques plages de Cuba: Académie Bulgare des Sciences, Bulletin de I'Institut de Zoologie (Sofia), v. 32, p. 151-158.

Golemansky, V., 1970f, Thécamoebiens (Rhizopoda, Testacea) nouveaux des eaux souterraines littorales de la Mer Noire: Acta Protozoologica (Warszawa), v. 8, no 2, p. 41-46.

Golemansky, V., 1970g, Chardezia caudata gen. n. sp. n. et Rhumberiella filosa gen. n. sp. n. Deux Thécamoebiens nouveaux du psammon littoral de la mer noire (Rhizopoda, Testacea): Académie Bulgare des Sciences, Bulletin de l'Institut de Zoologie et Musée (KH), v. 32, p. 121-125.

Golemansky, V., 1973a, Distribution horizontale des Thécamoebiens du psammon littoral des mers in DePuytorac, P. and Grain, J. (eds.), Progress in Protozoology. Abstracts of papers read at the Fourth International Congress on Protozoology, Clermont-Ferrand, 2-10 septembre 1973, p. 157; Université de Clermont (ClermontFerrand).

Golemansky, V., 1973b, Note sur la faune thécamoebienne (Rhizopoda, Testacea) du lac Morskie Oko en Tatras polonaises: B"Igarska Akademiya na Naukite, Izvestiya na Zoologicheskiya Institut s Muzei/Académie Bulgare des Sciences, Bulletin de I'Institut de Zoologie et Musée (Sofia), v. 38, p. 21-24.

Golemansky, V., 1973c, Deuxieme contribution a la connaissance des Thécamoebiens (Rhizopoda, Testacea) du psammal littoral de la Mer Baltique: Académie Bulgare des Sciences, Bulletin de I'Institut de Zoologie (Sophia), v. 38, p. 49-60.

Golemansky, V., 1973d, Messemvriella filosa n. gen. n. sp.- une nouvelle thécamoebienne psammobionte (Rhizopoda, Testacea) des eaux souterraines littorales de la Mer Noire: Zoologische Anzeiger, Leipzig, v. 190, no 5,6, p. 302-304.

Golemansky, V., 1974a, Rhizopodnata fauna (Rhizopoda, Testacea) na litorala i bentala na Popovo ezero v Pirin Planina, [French summary: La faune Rhizopodique (Rhizopoda, Testacea) du littoral et du benthal du lac Popovo dans la montagne de Pirine]: B"Igarska Akademiya na Naukite, Izvestiya na Zoologicheskiya Institut s Muzei/Académie Bulgare des Sciences, Bulletin de I'Institut de Zoologie et Musée (Sofia), v. 40, p. 47-58.

Golemansky, V., 1974b, Sur la composition et la distribution horizontale de l'association thécamoebienne (Rhizopoda, Testacea) des eaux souterraines littorales de la mer Noire en Bulgarie: B"Igarska Akademiya na Naukite, Izvestiya na Zoologicheskiya Institut s Muzei/Académie Bulgare des Sciences, Bulletin de 
I'Institut de Zoologie et Musée (Sofia), v. 40, p. 195-202.

Golemansky, V., 1974c, Lagenidiopsis valkanovi gen. n. sp. n.- un nouveau thécamoebien (Rhizopoda: Testacea) du psammal supralittoral des mers: Acta Protozoologica, v. 13, fasc. 1, p. 1-4, pl. 1.

Golemansky, V., 1974d, Psammonobiotidae fam. nov.- une nouvelle famille de thécamoebiens (Rhizopoda, Testacea) du psammal supralittoral des mers: Acta Protozoologica, v. 13, fasc. 11, p. 137-142, pls. 1, 2.

Golemansky, V., 1976a, Contribution à l'étude des Rhizopodes et des Héliozoaires du psammal supralittoral de la Méditerranée: Acta Protozoologica, v. 15, no 1, p. 35-45.

Golemansky, V., 1976b, Rhizopodes psammobiontes (Protozoa, Rhizopoda) du psammal supralittoral des côtes guinéennes de l'Atlantique: Acta Zoologica Bulgarica, v. 4 , p. 23-29.

Golemansky, V., 1977, Origine et évolution de l'association thécamoebienne du psammal supralittoral des mers in Hutner, S. H. (ed.), The Fifth International Congress of Protozoology, New York City 26 June- 2 July 1977. Abstracts of papers read at the Fifth International Congress on Protozoology, p. 359.

Golemansky, V., 1978, Adaptations morphologiques des thécamoebiens psammobiontes du psammal supralittoral des mers: Acta Protozoologica, v. 17, no 1, p. 141-152.

Golemansky, V., 1979a, Thécamoebiens psammobiontes du supralittoral coréen de la mer Japonaise et description de deux nouvelles espèces- Rhumbleriella coreana $\mathrm{n}$. $\mathrm{sp}$. et Amphorellopsis conica n. sp. (Rhizopoda, Testacea): Acta Zoologica Bulgarica, v. 12, p. 5-11.

Golemansky, V., 1979b, Thécamoebiens psammobiontes du supralittoral vietnamien de la Mer Chinoise et description de Cryptodifflugia brevicolla n. sp. (Rhizopoda: Arcellinida): Acta Protozoologica, v. 18, no 2, p. 285-292.

Golemansky, V., 1979c, Cyphoderia compressa n. sp. (Rhizopoda, Arcellinida) un nouveau thécamoebien psammbionte du supralittoral des mers: Acta Protozoologica, v. 18, p. $429-434$.

Golemansky, V., 1981a, Origine, relations philogénétiques (sic) et évolution des thécamoebiens interstitiels du supralittoral marin in: Dryl, S., Kazubski, S. L. and Ploszaj, J. (eds.): Progress in Protozoology. Abstracts of papers submitted to VI International Congress of Protozoology, Warszawa, Poland, July 5-11, 1981, p. 117; (Warszawa).

Golemansky, V., 1981b, Descrption de trois thécamoebiens (Protozoa: Rhizopodea) nouveaux des eaux souterraines littorales des mers: Acta Protozoologica, v. 20, no 1, p. 115-119. 
Golemansky, V., 1982a, Revision du genre Ogdeniella nom n. (=Amphorellopsis Golemansky, 1970) (Rhizopoda, Gromida) avec considerations sur son origine et evolution dansle milieu interstitiel: Acta Zoologica Bulgarica, v. 19, p. 3-12.

Golemansky, V., 1982b, Thécamoebiens (Protozoa: Rhizopodea) interstitiels du supralittoral Grec de la Mer Egée: Biologia Gallo-Hellenica, v. 9, no 2, p. 271-276.

Golemansky, V., 1983, Interstitial Thécamoebas (Rhizopoda, Arcellinida and Gromida) of the Soviet Finn Bay Coast: Acta Zoologica Bulgarica, v. 23, p. 1-8.

Golemansky, V., Couteaux, M.M., 1982, Étude en microscopie ?élecrtouigue à huit espèces de thécamoebiens interstitials du supralittoral mariu: Protistologica, v. 18, fasc. 4 , p. 473-480.

Golemansky, V., Ogden, C. G., 1980, Shell structure of three littoral species of testate amoebae from the Black Sea (Rhizopodes: Protozoa): Bulletin of the British Museum of Natural History (Zoology), v. 38, no 1, p. 1-6.

Golemansky, V.G. (1991) Thécamoebiens mésopsammiques (Rhizopoda: Arcellinida, Gromida \& Monothalamida) du sublittoral marin de l'Atlantique dans la région de Roscoff (France). Archiv für Protistenkunde, 140 35-43

Golemansky, V.G. (1992) Thécamoebiens interstitiels (Rhizopoda: Arcellinida, Gromida et Monothalamida) du supralittoral français de l'Atantique dans la région du Roscoff (Bai de Morlaix). Acta Zoologica Bulgaria, 45 3-13 


\section{GON-GUR}

Gong, X.X., Huazhong, S.Y., 1990, Studies on the protozoan fauna of Three-Gorge area in Changjiang (the Yangtze) River: Acta Hydrobiologica Sinica, v. 14, pp. 289297 (in Chinese).

Gosse, P. H., 1859, Evenings with the microscope: publ. (???), p. (???), (London).

Graaf, F. de, 1956, Studies on Rotatoria and Rhizopoda from the Netherlands.-I. Rotatoria and Rhizopoda from the "Grote Huisven": Biologisch Jaarboek Dodonaea (Gent), v. 23, p. 147-217.

Graaf, F. de, 1957, Hydrobiologisch Onderzoek van de Nederlandse Vennen: Natura Nr., v. 4, p. 41-46.

Graber, V., 1889/90?, title (?): Biologisches Centralblatt, v. 9, p. 729-735.

Gracia, M. del P., 1963, Resultados de la Expedición Peris-Alvarez a la isla de Annobón (Golfo de Guinea). VI. Tecamebas muscícolas: Publicaciones del Instituto de Biologia Aplicada Barcelona, v. 34, p. 5-16.

Gracia, M. del P., 1964, Tecamebas muscícoles del valle de Ribas (Gerona): Publicaciones del Instituto de Biologia Aplicada Barcelona, v. 39?, p. 123-127.

Gracia, M. del P., 1965a, Tecamebas muscícolas de Gran Canaria: Publicaciones del Instituto de Biologia Aplicada Barcelona, v. 38, p. 93-96.

Gracia, M. del P., 1965b, Nota sobre Tecamebas muscícoles de Ibiza (Islas Baleares): Publicaciones del Instituto de Biologia Aplicada Barcelona, v. 39, p. 109-111.

Gracia, M. del P., 1965c, Tecamebas muscícolas de Tenerife: Publicaciones del Instituto de Biologia Aplicada Barcelona, v. 39, p. 123-127.

Gracia, M. del P., 1966, Tecamebas muscícolas de Vallvidrera: Miscelánea Zoológica, v. 2, p. 3-6.

Gracia, M. del P., 1968a, Nota sobre Nebela carinata (Archer.) Leidy (Thecamoeboidea): Publicaciones del Instituto de Biologia Aplicada Barcelona, v. 44. p. $45-55$.

Gracia, M. del P., 1968b, Nota sobre algunas Tecamebas (Profozoos Rizópodos) de Nueva Guinea: Publicaciones del Instituto de Biologia Aplicada Barcelona, v. 45, p. 27-31.

Gracia, M. del P., 1971, Contribución al estudio morfológico y ecológica de las Tecamebas: p. 1-18; Universidad de Barcelona, Secretariado de Publicaciónes (Barcelona). 
Gracia, M. del P., 1972a, Contribución al estudio de los Tecamebas (Protozoa, Thecamoeboides). Tecamebas esfagnícolas de la Península Ibérica: Publicaciones del Instituto de Biologia Aplicada Barcelona, v. 52, p. 5-42.

Gracia, M. del P., 1972b, Contribución al estudio de los Tecamebas (Protozoa, Thecamoeboides). Tecamebas muscícolas de la Península lbérica: Publicaciones del Instituto de Biologia Aplicada Barcelona, v. 54, p. 5-16.

Gracia, M. del P., 1973a, Contribución al conocimiento de las Tecamebas (Protozoa, Thecamoeboidea. Estudio analítico y morfológico de los Tecamebas muscícolas: Publicaciones del Instituto d e Biologia Aplicada Barcelona, v. 54, p. 53-86.

Gracia, M. del P., 1973b, Ecologie des Thécamoebiens des tourbières pyrénéennes: in: DePuytorac, P. and Grain, J. (eds.), Progress in Protozoology. Abstracts of papers read at the Fourth International Congress on Protozoology, Clermont-Ferrand, 210 septembre 1973, p. 160; Université de Clermont (Clermont-Ferrand).

Gracia, M. del P., 1976, Ecología de las Tecamebas en las turberas pirenaicas: Miscelánea Zoológica (Barcelona), v. 3, no 5, p. 3-8.

Gracia, M. del P., 1978a, Tecamebocenosis de musgos aéreos de la isla de Mallórca: Publicaciones del Departamento de Zoología, Universidad de Barcelona, v. 3, p. 510.

Gracia, M. del P., 1978b, Distribución de las Tecamebas en la zona de bosque mediterraneo del Montseny: Miscelánea Zoologica, v. 4, p. 3-9.

Gracia, M. del P., 1980, Thécamoebiens de l'île de Tasmanie: Journal of Protozoology, v. 27, no 3, p. 87A-88A.

Grandori, L., 1934, Prime ricerche sui protozoi dei terreni e delle acque dell'alta montagna alpina (Piccolo San Bernardo): Bollettino del Laboratorio di Zoologia agraria e Bachicoltura del Reale Istituto Superiore Agrario di Milano, v. 4, fasc. 2, p. 14-42.

Grandori, R., Grandori, L., 1934, Studî sui Protozoi del terreno: Bollettino del Laboratorio di Zoologia agraria e Bachicoltura del Reale Istituto Superiore Agrario di Milano, v. 5, p. 1-341, pls. 1-14.

Gravenhorst, J. L. C., 1844, Naturgeschichte der Infusionsthierchen, nach Ehrenbergs grossem Werke über diese Thiere, in einer gedrängten vergleichenden Uebersicht dargestellt: p. I-VI, 1-60, Barth (Breslau).

Gray, T. R. G., Williams, S. T., 1971, Soil micro-organisms: in: University Reviews in Botany, p. I-VIII, 1-240, pls. 1-11; Oliver and Boyd (Edinburgh).

Greeff, R., 1866a, Ueber einige in der Erde lebende Amöben und andere Rhizopoden: Archiv fÜr Mikroskopische Anatomie, v. 2, no 2, 3, p. 299-331, pls. 17, 18. 
Greeff, R., 1866b, Zoologische Beobachtungen über neidere Thiere: Sitzungberichte des Naturwissenschaftlichen Veriens der Rheinland, v. 23, p. 10-12.

Greeff, R., 1870, Untersuchungen über Protozoen. II. Untersuchungen über Rhizopoden. 1. Ueber einen dem Bathybius haeckelii (Huxley) der Meerestiefen durch Vorkommen und Bau nahestehenden Organismus des süssen Wassers. 2. Ueber eine bei den Rhizopoden entdeckte wahrscheinlich geschlechtliche Fortpflanzung:

Verhandlungen des naturhistorischen Vereins der Preussischen Rheinlande und Westphalens (Bonn), (ser. 3, v. 7), 27p. + Appendix (?).

Greeff, R., 1888, Studien über Protozoen. Land-Protozoen: Sitzungsberichte der Gesellschaft zur Beförderung der gesammten Naturwissenschaften zu Marburg, v. 3, p. $90-158$.

Green, J., 1963, Zooplankton of the River Sokoto, the Rhizopoda Testacea: Zoological Society of London Proceedings, v. 141, p. 497-514.

Green, J., 1975, Freshwater ecology in the Mato Grosso, central Brazil, IV:

Associations of testate Rhizopoda: Journal of Natural History, v. 9, no 5, p. 545560.

Green, J., 1996, Associations of testate rhizopods (Protozoa) in the plankton of a Malaysian estuary and two nearby ponds: Journal of Zoology, v. 239, pp. 485-506

Green, J., Corbet, S. A. Watts, E., Oey, B. L., 1976, Ecological studies on Indonesian lakes overturn and restrastification of Ranu Lamongan: Journal of Zoology (London), v. 180 , no 3 , p. $315-354$.

Griffin, J. L., 1972, Movement, fine structure, and fusion of pseudopods of an enclosed amoeba, Diffluqiella sp.: Journal of Cell Science, v. 10, p. 563-583.

Griffith, J. W., Henfrey, A., 1855-1856, The micrographic dictionary, v. 1 (1855), v. 2 (1856), (London).

Griffith, J. W., Henfrey, A., Jones, T. R., 1883, The micrographic dictionary, 4th edition: 2 vols.: p. 1-XLVI + 1-829, (london).

Groenlund, T., Lortie, G., Guilbault, J.P., Bouchard, M.A., Sarnisto, M., - 1990 Diatoms and arcellaceans (thecamoebians) from the Lac du Cratere du NouveauQuebec, Ungava, Quebec: Canadian Journal of Botany - Journal Canadien de Botanie???, v. 68, pp. 1187-1200.

Groenlund, T., Lortie, G., Guilbault, J.P., Bouchard, M.A., Sarnisto, M., anno?? Diatoms and arcellaceans (thecamoebians) from the lac du cratere du NouveauQuebec, Ungava, Quebec, Canada.: In: L'Histoire naturelle du cratere du Nouveau Quebec, Bouchard, M.A. and , Peloquin, S. (eds.), Collection Environnement et Geologie. v. 7, pp. 277-313, Section du Genie de l'Environnement de l'Ecole Polytechnique de Montreal. 
Grospietsch, T. 195(?), Zur Rhizopodenfauna Schwedisch-Lapplands: p. 55-56, in: Groupement d'études sur les Thécamoebiens, 195(?): Communiqué du Groupement d'Etudes sur les Thécamoebiens: Protistologica, v. 13, no 3, p. 333-334.

Grospietsch, T., 195(?). Rhizopoden-Analyse der Moore: in: Bericht über den VI. Internationalen Kongress für Moorforschung, vom 5. bis 9. Juni 1958 in Brüssel und Spa, p. 175-182.

Grospietsch, T., 1952, Die beschalten Amöben unserer Hochmoore: Mikrokosmos), v. 41 , no 10 , p. 219-224, Stuttgart.

Grospietsch, T., 1952b, Die Rhizopodenanalyse als Hilfsmittel der Moorforschung: Naturwissenschaften, v. 39 , no 14 , p. 318-323

Grospietsch, T., 1953a, Die Untersuchung von Mooren mit Hilfe der Rhizopodenanalyse: Mikrokosmos, v. 42, no 5, p. 101-106.

Grospietsch, T., 1953b, Rhizopodenanalytische Untersuchungen an Mooren Ostholsteins: Archiv für Hydrobiologie, v. 47, no 3, p. 321-452.

Grospietsch, T., 1954a, Studien über die Rhizopodenfauna von Schweidisch Lappland: Archiv für Hydrobiologie, v. 49, n167 4, p. 546-580.

Grospietsch, T., 1954b, Die bedeutung der Rhizopodenanalyse für die oorforschung: Mitteilungen aus der Max-Planck-Gesellschaft, v. 2, p. 94-97.

Grospietsch, T., 1955, Die testaceen Rhizopoden der Hochmoore und ihre Bedeutung für die Moorforschung: Gewässer und Abwässer, v. 6, p. 5-19, (Düsseldorf) - [reprints bears date 1954/55]

Grospietsch, T., 1957, Beitrag zur Rhizopodenfauna des Lago Maggiore: Archiv für Hydrobiologie, v. 53, n167 3, p. 323-331.

Grospietsch, T., 1958a, Beiträge zur Rhizopodenfauna Deutschlans. I. Die Thekamöben der Rhön: Hydrobiologia, v. 10, p. 305-322.

Grospietsch, T., 1958b, Wechseltierchen (Rhizopoden): Kosmos-Gesellschaft der Naturfreunde/Franckh'sche Verlagshandlung; Sammlung " Einführung in die Kleinlebewelt", p. 1-82, pl. 1-4, (Stuttgart).

Grospietsch, T., 1964, Die Gattungen Cryptodifflugia und Difflugiella (Rhizopoda Testacea): Zoologische Anz., v. 172, no 4, p. 243-257.

Grospietsch, T., 1965a, Monographische Studie der Gattung Hyalosphenia Stein (Rhizopoda Testacea): Hydrobiologia, v. 26, p. 211-241.

Grospietsch, T., 1965b, Schalenamöben im Boden: Mikrokosmos, v. 54, p. 14-18.

Grospietsch, T., 1967, Die Rhizopodenanalyse der Moore und ihre 
Anwendungsmöglichkeit: in: Tüxen, R. (ed.): Pflanzensoziologie und Palynologie. Bericht über das Internationale Symposium in Stolzenau/Weser, 1962, der Internationalen Vereinigungf für Vegetationskunde, p. 181-192; Dr. W.Junk (Den Haag).

Grospietsch, T., 1970, Rhizopoda: in: Gray, P. (ed.): The Encyclopedia of the Biological Sciences: p. 810-812; Van Nostrand Reinhold Co. (New York)

Grospietsch, T., 1971, Rhizopoda, Beitrag zur Ökologie der testaceen Rhizopoden von Marion Island, in: van Zinderen Bakker, E.M.Sc., Winterbottom, J.M. and Dyer R.A. (eds): Marion and Prince Edward Islands. Report on the South African Biological and Geological expedition 1965/1966: p. 411-423, 89 photos; A.A.Bolkema (Cape Town).

Grospietsch, T., 1972a, I. Protozoa.A. Testacea und Heliozoa: in: Elster, H.J.and Ohle, W. (eds.): Die Binnengewässer; v. 26: Das Zooplankton der Binnengewässer, 1. Teil, p. 1-30; E. Schweizerbart (Stuttgart).

Grospietsch, T., 1972b, Neue Ergebnisse der Rhizopodenanalyse für die Moorforschung: in: Sládecek, V, (ed.): Congress in USSR 1971: Internationale Verein für theoretische und angewandte Limnologie. Verhandlungen, v. 18, p. 1031-1038.

Grospietsch, T., 1975, Beitrag zur Kenntnis der Testaceen-Fauna des Lago Valencia (Venezuela): in: Sládecek, V. (ed.), Congress in Canada 1974: XI. Ecology of Aquatic Organisms. 3. Animals: Verh. internat Vereins theor. angew Limnol. v. 19, part 4, p. 2778-2784.

Grospietsch, T., 1976, Rhizopodenanalytische Untersuchungen in zwei Mooren im oberbayerischen Jungmoränengebiet südlich des Starnberger Sees (Weidfilz und Schögenfilz): Telma (Hanover), v. 6, no 4, p. 25-34.

Grospietsch, T., 1979a, Informative Bulletin of Testacean Workers, no 4, p. 1-61.

Grospietsch, T., 1979b, List of the new species and subspecies (also new variations and forms) describes, a. til (sic) 1975; b. in 1976; c. in 1977; d. 1978: Informative Bulletin of Testacean Workers, no 4, p. 25-27.

Grospietsch, T., 1979c, Appendix: List of the slide collection made by E. Penard which is deposited in the British Museum (Register numbers 20.12.8.1-706): Informative Bulletin of Testacean Workers, no 4, p. 30-61.

Grospietsch, T., 1982a, The thermal lake of Héviz (Hungary) and its fauna of Rhizopod Testacea (Der Thermalsee von Héviz (West Ungarn) und seine Testaceen-Fauna): Arch. Hydrobiol. v. 95, no 1-4, p. 93-105.

Grospietsch, T., 1982b, Informative Bulletin of Testacean Workers, no 5, p. ???.

Gruber, A., 1880, Fortpflanzung bei Euglypha alveolata: Zoologischer Anzeiger, v. 3, 
p. 582-584 -[summarized in Gruber, 1881a].

Gruber, A., 1881a, Reproduction of Euglypha alveolata: Journal of the Royal Microscopical Society, ser. 2, v. 1, part 2, p. 69.

Gruber, A., 1881b, Der Theilungsvorgang bei Euglypha alveloala: Zeitschrift für wissenschaftliche Zoologie, v. 35, p. 431-439 - [summarized in Gruber, 1882a].

Gruber, A., 1881c, Die Theilung der monothalamen Rhizopoden: Zeitschrift für wissenschaftliche Zoologie, v. 36, no 1, p. 104-124, pls. 4, 5.

Gruber, A., 1881d, Beiträge zur Kenntniss der Amöben: Zeitschrift für wissenschaftliche Zoologie, v. 36, no 3, p. 459-470, pl. 30 - [translated in Gruber, 1882b].

Gruber, A., 1881e, Der Teilungsvorgang bei den Rhizopoden: Biologisches Zentralblatt, v. 1, p. 456-459.

Gruber, A., 1881f, Ein Wurzelfusser des Susswassers in Bau und Lebenerscheinungen dargestellt: in: Zacharias, O., Tier- und Pflanzenwelt des Susswassers, v. 2, p. 135162.

Gruber, A., 1881g, title (?): Archives des Sciences Physiques et Naturelles, sér. 3, v. 6, p. 624-627.

Gruber, A., 1881h, title (?): Biologisches Centralblatt, v. 1, p. 79-80.

Gruber, A., 1882a, On the phenomena of division in Euglypha alveolata and the monothalamous Rhizopods in general: Annals and Magazine of Natural History, ser. 5, v. 9 , no 50, p. $135-137$.

Gruber, A., 1882b, Contributions to the knowledge of the Amoebae: Annals and Magazine of Natural History, ser. 5, v. 9, no 50, p. 106-116, pl. 9.

Gruber, A., 1882c, Untersuchungen über einige Protozoen: Zeitschrift für Wissenschaftliche Zoologie, v. 38, p. 45-70, pl. 2-4 - [translated in Gruber, 1883b; summarized in Gruber, 1883c].

Gruber, A., 1883a, Ueber Kerntheilungsvorgänge bei einigen Protozoen: Zeitschrift für Wissenschaftliche Zoologie, v. 38, no 3, p. 372-391.

Gruber, A., 1883b, Investigations upon some Protozoa: Annals and Magazine of Natural History, ser. 5, v. 11, no 64, p. 266-276, no 65, p. 315-326, pl. 13.

Gruber, A., 1883c, Observations on Protozoa: Journal of the Royal Microscopical Society, ser. 2, v. 3, part 1, p. 222-223.

Gruber, A., 1884a, Die Protozoen des Hafens von Genua: Nova Acta Academiae Caesareau Leopoldino-Carolinae Germanicae Naturae Curiosorum 
[Verhandlungen der Kaiserlichen Leopoldino-Carolina Deutschen Akademie der Naturforscher], v. 46, p. 473-539, pls. 7-11.

Gruber, A., 1884b, Ueber Kern und Kernteilung bei den Protozoen: Zeitschrift für Wissenschaftliche Zoologie, v. 40, no 1, p. 121-153, pls. 8, 9 - [summarized in Gruber 1884c].

Gruber, A., 1884c, ??title: Biologisches Centralblatt, v. 4, p. 233-235.

Gruber, A., 1886, Die Frage nach dem Bestehen verschiedener Plasmaschichten im Weichkörper der Rhizopoden: Biologisches Centralblatt, v. 6, p. 5-8.

Gruber, A., 1888a, Kleinere Miltheilungen über Protozoen-Studien: Bericht der Naturforschende Gesellschaft zu Freiburg i. Br., v. 2, p. 149-162, pl. 6; [summarized in Gruber, 1888b]

Gruber, A., 1888b, Notes on Protozoa: Journal of the Royal Microscopical Society, part 2, p. 969-970. [written report of a verbal presentation]

Gruber, A., 1891, Ein Wurzelfüsser des Süsswassers in Bau und Lebenserscheinungen: in: Zacharias, $O$. (ed.), Die Tier- und Pflanzenwelt des Süsswassers, v. 1, p. 140-(???) (Leipzig).

Gruber, A., 1893, Amöben-Studien: Bericht der Naturforschende Gesellschaft zu Freiburg i. Br., v. 8, p. 24-34.

Gruithuisen, F. von P., 1818, Physiologische und physiographische Bemerkungen über mikroskopische Thiere, besonders in Hinsicht ihrer Entstehung: in: Ehrhart, J. N. (ed.), Medicinisch-chirurgische Zeitung, p. (???). (Salzburg).

Gruithuisen, F. von P., 1820, Physiologische und physiographische Bemerkungen über mikroskopische Thiere, besonders in Hinsicht ihrer Entstehung: in: Oken, L. (ed.), Isis, columns 247-260.

Guenther, K., 1927, Die Welt der Urtierchen: in: (?), p. 1-118, Ulstein (Berlin).

Guerne, J. de, 1888, Excursions zoologiques dans les îles de Fayal et de San Miguel: no further information - (Paris).

Güntert, A., 1925, Über postglaziale Rhizopodenfunde: in: Bosch, R., et al., Prähistorisches und Naturwissenschaftliches vom Hallwilersee: Mitteilungen der Aargauischen Naturforschenden Gesellschaft, v. 17, p. (?).

Gurvich, V. V., 1975, Formation of Rhizopoda: Testacea taxocenoses in the Kachovka Resevoir: Acta Protozoologica, v. 14, p. 297-311. 


\section{HAE-HER}

Haeck, M.Cl., 1956, Bijdrage tot de kennis der drentse Rhizopoda Testacea en Heliozoa: Biologische Jaarboek, "Dodonaea" (Gent), v. 23, p. 254-264.

Haeckel, E., 1861, De Rhizopodum finibus et ordinibus: 16p, (Berolini).

Haeckel, E., 1866 (1868, 1869?), Generelle Morphologie der Organismen. Allgemeine Grundzüge der organischen Formen-Wissenschaft, mechanisch begründet durch die von Charles Darwin reformirte Descendenz-Theorie, [2 volumes: I. Band: Allgemeine Anatomie der Organismen. Kritische Grundzüge der mechanischen Wissenschaft von den entwickelten Formen der Organismen, begründet durch die Descendenz-Theorie, p. I-XXXII, 1-574; II. Band: Allgemeine Entwicklungsgeschicte der Organismen. Kritische Grundzüge der mechanischen Wissenschaft von den entwickelten Formen der Organismen, begründet durch die Descendenz-Theorie], p. I-CLX + 1-462; Georg Reimer (Berlin).

Haeckel, E., 1870a, Ueber Entwicklungsgang und Aufgabe der Zoologie: Jenaische Zeitschrift für Medizin und Naturwissenschaft, v. 5, p. 353-370. - [text contained also in Haeckel 1870c and 1879b].

Haeckel, E., 1870b, Beiträge zur Plastiden-Theorie: Jenaische Zeitschrift für Medizin und Naturwissenschaft, v. 5, p. 492-550. - [text contained also in Haeckel 1870c].

Haeckel, E., 1870c, Biologische Studien. Erstes Heft: Studien über Moneren und andere Protisten, nebst einer Rede über Entwickelungsgang und Aufgabe der Zoologie: p. 1-184, pls. 1-4, W. Engelmann (Leipzig).

Haeckel, E., 1871, Nachträge zur Monographie der Moneren: Jenaische Zeitschrift für Medizin und Naturwissenschaft, v. 6, p. 23-44. - [text contained also in Haeckel 1870c].

Haeckel, E., 1878a, Das Protistenreich. Eine populäre Uebersicht über das Formengebiet der niedersten Labewesen: Kosmos (Leipzig), v. 3, p. (?). [translated in Haeckel, 1879a and 1880]

Haeckel, E., 1878b, Das Protistenreich. Eine populäre Uebersicht über das Formengebiet der niedersten Labewesen. Mit einem wissenschaftlichen Anhange: System der Protisten: p. 1-104, E. Günther (Leipzig). [translated in Haeckel, 1879a and 1880].

Haeckel, E., 1879a, Le Règne des Protistes. Aperçu sur la morphologie des êtres vivants les plus inférieurs, suivi de la classification des protistes. Traduit de l'allemand et précédé d'une introduction par J. Soury: p. I-LXIV, 1-121, C. Reinhold (Paris).

Haeckel, E., 1879b, Gesammelte populäre Vortäge aus dem Gebiete der Entwicklungslehre. Zweites Heft. p. (?), E. Strauss (Bonn). 
Haeckel, E., 1879c, Natürliche Schöpfungsgeschichte, p. I-XXXII, 1-718, G. Reimer (Berlin).

Haeckel, E., 1880, Le Règne des Protistes: - no further information.

Haeckel, E., 1894, Systematische Phylogenie. Entwurf eines natürlichen Systems der Organismen auf Grund ihrer Stammesgeschichte. 1. Theil: Systematische Phylogenie der Protisten und Pflanzen: p. I-XV + 1-400, Georg Reimer (Berlin).

Haeckel, E., 1906, Prinzipien der generellen Morphologie der Organismen: p. I-XVI, 1-447, Georg Reimer (Berlin). [the text is a partial reprint of Haeckel, 1866]

Hall, R. P., Loefer, J. B., 1930, studies on Euglypha. I. Cytoplasmic inclusions of Euglypha alveolata: Archiv für Protistenkunde, v. 72, p. 365-376.

Hallas, T. E., 1975, Notes on the encystation in Microcorycia radiata (Testacea lobosa, Protozoa): Pedobiologia, v. 15, no 2, p. 149-150.

Haman, D, 1986, Testacea lobosa from Big Bear Lake, California, with comments on Difflugia tricuspis Carter, 1856: Revista Española de Micropaleontologia, v. 18, pp. 47-54.

Haman, D., 1982a, Modern Thechamoebinids (Arcellinida) from the Balize Delta, Louisiana: Transactions, Gulf Coast Association of Geological Societies, v. 32, p. 353-376, pls. 1-4.

Haman, D., 1982b, Modern Thechamoebinids (Arcellinida) from the Balize Delta, Louisiana: American Association of Petroleum Geologists Bulletin, v. 69, no 9, p. 1430-1431.

Haman, D., 1986, Testacealobosa from Big Bear Lake, California, with comments on Difflugia tricuspis Carter, 1856: Revista Espaola de Micropaleontología, v. XVIII, no 1, p. 47-54.

Haman, D., 1988, Annotated lexicon of testate lobosid and filosid amoebean genera, subgenera on type species (Sarcodina, Protozoa, "thecamoebians"): Revista Española de Micropaleontologia, v. 20, 2, pp. 205-244

Haman, D., 1990, Living thecamoebinid distribution, biotopes and biofacies, in an upper deltaic plain lacustrine subenvironment, Lac des Allemands, Louisiana: Revista Española de Micropaleontologia, v. 22, pp. 87-100.

Hanson, E. D., 1967, Protozoan development: in: Florkin, M. and Scheer, B. T. (eds.), Chemical zoology; v. 1: Kidder, G. W. (ed.), Protozoa, p. 395-539, Academic Press (New York).

Hanson, E. D., 1977, The origin and early evolution of animals: p. I-X, 1-670, Wesleyan University Press (Middletown, Conn) and Pitman (London). 
Harnish, 0., 1948, Rhizopodenanalyse der Moore: Biologisches Zentralblatt, v. 67, p. 551-562.

Harnish, O., 1924, Studien zur Okologie der Moorfauna: Biologisches Zentralblatt, v. 44, p. 110-127.

Harnish, O., 1925a, Studien zur Ökologie und Tiergeographie der Moore: Zoologisch Jahrbuch (Abteilung Systematik), v. 51, p. 1-166.

Harnish, O., 1925b, Die Beziehungen der mitteleuroppaischen Tier welt zue Eiszeit: Archiv für Hydrobiologie, v. 15, p. 512-525.

Harnish, O., 1929a, Einige Daten zur rezenten und fossilen testaceen Rhizopodenfauna der Sphagnen, Archiv für Hydrobiologie, v. 18, p. 345-360.

Harnish, O., 1929b, Biologie der Moore: in: Die Binnengewässer, Band 7, 146p (Stuttgart).

Harnish, O., 1932, Die testaceen Rhizopoden der deutschen limnologischen SundaExpedition: Archiv für Hydrobiologie, Supplementband 11, p. 578-595.

Harnish, O., 1937, Neue daten zur testaceen Rhizopodenfauna nicht moorbildender Sphagnete: Zoologischer Anzeiger, v. 120, p. 129-137.

Harnish, O., 1938, Weitere daten zur Rhizopodenfauna Lapplands: Zoologischer Anzeiger, v. 124, p. 138-150.

Harnish, O., 1950 (?), Daten zur Gestaltung der oekologischen Valenz der sphagnicolen Rhizopoden im Verlauf des Postglazials: Deutsche Zoologische Zeitschrift (Hannover), v. 1, p. 222-233.

Harnish, O., 1951a. Rhizopodenanalytische studien an einigen Vogesenmooren: Archiv für Hydrobiologie, v. 45, p. 332-345.

Harnish, O., 1951b, Bemerkungen zu meiner Bearbeitung der testaceen Rhizopoden der deutschen limnologischen Sunda-Expedition: Archiv für Hydrobiologie, Supplementband 19, p. 627-629.

Harnish, O., 1958, II. Klasse: Wurzelfüssler, Rhizopoda: in: Brohmer, P., Ehrmann, P. and Ulmer, G. (eds.), Die Tierwelt Mitteleuropas, Band 1: Urtiere-Hohltiere-Würmer, Lieferung 1b, p. 1-75, pls. 1-26; Quelle and Meyer (Leipzig).

Harrison, F. W., 198?, ?title: in: Simpson, T. and Volcani, B. (eds.), Biology of Silicon, ?pages, ?publisher.

Harrison, F. W., Dunkelberger, D., Watabe, N., Stump, A. B., 1975, The cytology of the testaceous rhizopod Lesquereusia spirilis (Ehrenberg) Penard. I. Cytochemistry: Acta Histochemica, v. 54, no 1, p. 71-77. 
Harrison, F. W., Dunkelberger, D., Watabe, N., Stump, A. B., 1976, The cytology of the testaceous rhizopod Lesouereusia spiralis (Ehrenberg) Penard. I. Ultrastructure and shell formation: Journal of Morphology, v. 150, no 2, part 1, p. 343-358, pls. 1-4.

Hartman, M., 1907, Das system der Protozoen. Zugleich vorläufige Mitteilung über Proteosoma (Labbé): Archiv für Protistenkunde, v. 10, p. 139-158.

Hartman, M., 1913, Rhizopoda: in: Handwörterbuch der Naturwissenschaften, Band 8, p. 422-446; Gustav Fischer (Jena).

Hartman, M., 1928, Über experimental Unsterblichkeit von Protozoen individuen. Ersatz der Fortpflanzung von Amoeba proteus durch fortgesetze regenerationen: Zoologische Jarbucher Abteilung für Allgemeine Zoologie und Physiologie der Tier, v. 45, p. ??.

Hartog, M., 1906, Protozoa: in: Harmer, S. F. and Shipley, A. E. (eds.), The Cambridge Natural History, v. 1, p. 1-162; Macmillan and Co., Ltd. (London).

Hartog, M., 1911, Rhizopoda: in: Enclclopedia Britannica, 11th edition, v. 23, p. 245248; University Press (Cambridge).

Harvey, F. L., 1888, Contribution to the fresh-water Rhizopods: American Naturalist, v. 22 , p. $71-74$.

Hauer-Eichardt, H., 1954, Das zooplankton in den seen des Sudlichen Schwarzwaldes: Archiv für Hydrobiologie, Suppliment to v. 20?, p. 304-374.

Hausmann, E., 1975, Die Regeneration der Zelloberfläche bei Hvalodiscus simplex: Protistologica, v. 11, no 2, p. 135-147.

Hayward, B.W., Grenfell, H., Cairns, G., Smith, A. (1996) Environmental controls on benthic foraminiferal and thecamoebian associations in a New Zealand tidal inlet. Journal of Foraminiferal Research, 26 150-171.

Heal, 0. W., 1964a, Observations on the seasonal and spatial distribution of Testacea (Protozoa: Rhizopoda) in Sphagnum: Journal of Animal Ecology, v. 33, p. 395-412.

Heal, O. W., 1962, The abundance and micro-distribution of testsate amoeba (Rhizopoda: Testacea) in Sphagnum: Okios, v. 13, no 1, p. 35-47.

Heal, O. W., 1963a, Morphological variation in certain Testacea (Protozoa: Rhizopoda): Archiv für Protistenkunde, v. 106, p. 351-368.

Heal, O. W., 1963b, Soil fungi as food for amoeba: in: Doeksen, J. and Drift, J. van der (eds.), Soil Organisms. Proceedings of the Colloquium on soil fauna, soil microflora and their relationships, Oosterbeek, the Netherlands, September 10-16, 1962, p. 289-297; North-Holland (Amsterdam).

Heal, O. W., 1964b, The use of cultures for studying Testacea (Protozoa: Rhizopoda) in 
soil: Pedobiology, v. 4, p. 1-7.

Heal, O. W., 1965a, Observations on testate amoeba (Protozoa: Rhizopoda) from Signy Island, South Orkney Islands: Bull. Brit. Antarct. Surv., v. 6, p. 43-47.

Heal, O. W., 1965b, Some slide collections of Protozoa, especially Testacea: Journal of the Quekett Microscopical Club, ser. 4, v. 30, p. 1-6.

Heal, O. W., 1970, Methods of study of soil Protozoa: in: Methods of study in soil ecology. Proceedings of the Paris Symposium on ecology and conservation, organized by UNESCO and the International Biological Programme, p. 119-125; UNESCO publication (Place de Fontenoy, Paris).

Heal, O. W., 1970: Methods of study of soil Protozoa: ???journal, p. 119-126.

Heal, O., 1961, The distribution of testate amoeba (Rhizopoda, Testacea) in some fens and bogs in northern England: Journal of the Linnean Society of London, Zoology, v. 44, p. $369-382$.

Hedley, R. H., Bertaud, W. S., 1962, Electron microscope observations of (Sarcodina). Journal of Protozoology, v. 9, no 1, p.79-87.

Hedley, R. H., Ogden, C. G., 1973, Biology and fine structure of Euglypha rotunda (Testacea: Protozoa): British Museum of Natural History Bulletin (Zoology), v. 25, no 4, p. 119-137, pl. 1-7.

Hedley, R. H., Ogden, C. G., 1974a, Observations on Trinema lineare Penard (Testacea: Protozoa): British Museum of Natural History Bulletin (Zoology), v. 26, p. 187-199.

Hedley, R. H., Ogden, C. G., 1974b, Adhesion plaques associated with the production of a daughter cell in Euglypha (Testacea: Protozoa): Cell Tiss. Res., v. 153, p. 261268.

Hedley, R. H., Ogden, C. G., Krafft, J. I., 1974, Observations on the clonal cultures of Euglypha acanthopora and Euglypha strigosa (Testacea: Protozoa): British Museum of Natural History Bulletin (Zoology), v. 27, p. 103-111.

Hedley, R. H., Ogden, C. G., Mordan, N. S., 1976, Manganese in the shell of Centropyxis (Rhizopodea: Protozoa): Cell Tiss. Res., v. 171, no 4, p. 543-549.

Hedley, R. H., Ogden, C. G., Mordan, N. S., 1977, The biology and fine structure of Cryptodifflugia oviformis (Rhizopodea: Protozoa): British Museum of Natural History Bulletin (Zoology), v. 30, no 8, p. 311-328, pl. 1-6.

Hedley, R. H., Wakefield, J. St. J., 1969, Fine structure of Gromia oviformis (Rhizopodea: Protozoa): British Museum of Natural History Bulletin (Zoology), v. 18, p. 69-89, pl. 1-12. 
Hedley, R.H., 1962, Gromia oviformis (Rhizopodea) from New Zealand with comments on the fossil Chitinozoa: New Zealand Journal of Science, v. 5, pp. 121-136.

Hegner, R. W., 1918, Variation and heredity durring the vegetative reproduction of Arcella dentata: Proceedings of the National Academy of Science, v. 4, no 9, p. 283288.

Hegner, R. W., 1919a, Quantitative relations between chromatin and cytoplasm in the genus Arcella, with their relations to external characters: Proceedings of the National Academy of Science, v. 5, no 1, p. 19-22.

Hegner, R. W., 1919b, Heredity, variation, and the appearance of diversities during the vegetative reproduction of Arcella dentata: Genetics, v. 4, p. 95-150.

Hegner, R. W., 1919c, The effect of environmental factors upon the heritable characteristics of Arcella dentata and A. polypora: Journal of Experimental Zoology, v. 29, p. $427-441$.

Hegner, R. W., 1920, The relations between nuclear number, chromatin mass, cytoplasmic mass and shell characteristics in four species of the genus Arcella: Journal of Experimental Zoology, v. 30, no 1, p. 1-95.

Heinis, F. K., 1911, Beitrag zur Kenntnis der Zentalomerikanischen Moosfauna: Annales de la Société de Zoologie et du Muséum d'Histoire Naturelle de Genève, v. 19 , p. 253-266, 1 pl.

Heinis, F. K., 1928-1929 (?), Die Moosfauna der Krakatau: Truebia, v. 10, p. 231-244, 1 pl.

Heinis, F., 1908, Beitrag zur Kenntnis der Moosfauna der Kanarischen Inseln: Zoologischer Anzeiger, v. 33, p. 711-716.

Heinis, F., 1910, Systematik und Biologie der Moosbewohnenden Rhizopoden, Rotatorien und Tardigraden der Umgebung von Basel mit Berücksichtigung der übrigen Schweiz: Archiv für Hydrobiologie, v. 5, p. 89-166, 217-256.

Heinis, F., 1911a, Beiträge zur Kenntnis der Centralafrikanischen Moosefauna: Revue Suisse de Zoologie, v. 19, p. 253-256, pl. 4.

Heinis, F., 1911b, Über die Mikrofauna am Bolchen: Naturforschende Verhandlungen Gesellschaft Basel, v. (?), p. 81-96.

Heinis, F., 1914, Die Moosfauna Columbiens: in: Voyage d'exploration scientifique en Colombie; Mémoires de la Société Neuchateloise des Sciences Naturelles, v. 5, p. $675-730$.

Heinis, F., 1920, Über die Mikrofauna alpiner Polster- und Rosettenpflanzen:

Festschrift fur Zschokke, v. 6, p. 1-29. 
Heinis, F., 1933, Beiträge zur Mikrofauna der Unigebung von Leistal. IX:

Naturforschende Gesellschaft Basel, v. (?), p. 1-8.

Heinis, F., 1937, Beiträge zur Mikrobiozonose in Alpinen Pflanzenpolstern: Berichte Geobotanische Forschungsinstitut Rubel in Zurich, fur den Jahn 1936, v. (?), p. 61-76.

Heinis, F., 1945, Beiträge zur Mikrobiozonose der Sphagnumpolster auf God del Fourn im Nationalpark: Ergebnisse Wissenschaften Unters. Schwiez. Nationalparkes N. S., v. 1, p. 527-547.

Hempel, A., 1898, A list of the Protozoa and Rotifera found in the Illinois River and adjacent lakes at Havana, III.: Illinois State Laboratory of Natural History Bulletin, v. 5, art. 6, p. 301-388.

Hendon, D.,Charman, D.J. (1997) The preparation of testate amoebae (Protozoa: Rhizopoda) samples from peat. The Holocene, 7 199-205.

Henneguy, L. F., 1896, Leçons sur la cellule: morphologie et reproduction: ??journal, pages (Paris).

Hertwig, R., 1879a, Der Organismus der Radiolarien: Denkschriften der Medizinischnaturwissenschaftlichen Gesellschaft zu Jena (=Jenaische Denkschriften), v. 2, no 3, p. I, II, 129-277, pls. VI-XV.

Hertwig, R., 1879b, Der Organismus der Radiolarien: p. I-IV, 1-149, pls. I-X.

Hertwig, R., 1887, Über Kernteilung bei Infusorien: Sitzungsberichte der Gesellschaft für Morphologie und Physiologie in München, v. 3, p. 127-128.

Hertwig, R., 1891, Lehrbuch der Zoologie: v. 1, p. (?) (Jena).

Hertwig, R., 1892, Über Befruchtung und Conjugation: Verhandlungen der deutschen zoologischen Gesellschaft, v. 2, p. 95-113.

Hertwig, R., 1893, Lehrbuch der Zoologie: 2 Auflage, 576 p. (Jena)

Hertwig, R., 1899, Über Encystierung und Kernvermebrung bei Arcella vulgaris: in:

Festschrift zum siebenzigsten Geburtstag von Carl von Kupfer: p. 367-382, pls. 3739 in separate Atlas, Gustav Fischer (Jena).

Hertwig, R., 1900, Lehrbuch der Zoologie: 5 Auflage, p. (?), (Jena).

Hertwig, R., 1903, A manual of zoology: p. (?) [translated by J.S.Kinsley] (London).

Hertwig, R., 1919, Lehrbuch der Zoologie: 12 Auflage, 686 p. (Jena).

Hertwig, R., Lesser, E., 1874, Über Rhizopoden und denselben nahestehende Organismen: Archiv für mikroskopische Anatomie, v. 10 (Supplement), p. 35-243, 
pls. 2-5. 


\section{HES-KOR}

Hesmer, H., 1929, Microfossilien in Torfen: Paläontologische Zeitschrift: v. 11, p. 245-257.

Hickson, S.J., 1909, The Protozoa: Section D - The Lobosa: in: Lankester, E. R. (ed.): A treatise on zoology: Part I: Introduction and Protozoa: 1st fasc., p. 68-93, A. and C. Black (London).

Hill, J. C., 1933, The Golgi apparatus of Protozoa: Journal of the Royal Microscopical Society, ser. 3, v. 53, no 3, p. 227-247.

Hitchcock, R., 1881, Synopsis of the fresh-water Rhizopods: p. i-viii + 1-56, New York.

Hitlerman, H., Haman, D., 1985, Sociology and Synecology of brachish-water foraminifera and Thecamoebinids of the Balize Delta, Louisiana: Facies, v. 13, p. 287294.

Honiberg, B.M., Balamuth, W. (1963) Subphylum Sarcomastigophora nom. Nov. to embrace the flagellate and amoiboid assemblages of protozoans. Journal of Protozoology 10 (suppl.) 27.

Honig, C.A., Scott, D.B., 1987, Postglacial stratigraphy and sea-level change in southwestern New Brunswick: Canadian Journal of Earth Sciences, v. 24, pp. 354364.

Honigman, H., 1909, Beiträge zur Kenntnis des Süsswasserplanktons: Abhandlungen aus dem Museum der Magdeburg, v. 2, no 1, p. 49-87, pl. 2.

Hoogenraad, H. R., 1907a, Einige Beobachtungen an Vampyrella lateritica LEIDY: Archiv für Protistenkunde, v. 8, p. 216-224.

Hoogenraad, H. R., 1907b, Zur Kenntnis von Hyalodiscus rubicundus Hertwig und Lesser: Archiv für Protistenkunde, v. 9, p. 84-99.

Hoogenraad, H. R., 1908a, Rhizopoden en Heliozoën vit het zoetwater van Nederland. I: Tijdschrift der Nederlansche Dierkundige Vereeniging, ser. 2, v. 10, no 4, p. 384424.

Hoogenraad, H. R., 1908b, Bemerkungen über einige Süsswasse-rhizopoden und Heliozoen: Annales de Biologie Lacustre, v. 3, no 2, p. 248-258.

Hoogenraad, H. R., 1914, Rhizopoden en Heliozoën vit het zoetwater van Nederland. III: Tijdschrift der Nederlansche Dierkundige Vereeniging, ser. 2, v. 13, no, p. (?).

Hoogenraad, H. R., 1927, Zur Kenntnis der Fortpflanzung Paulinella chromatophora: Zoologischer Anzeiger, v. 72, p. 140-150. 
Hoogenraad, H. R., 1933, Einige Beobachtungen an Bulinella indica Penard: Archiv für Protistenkunde, v. 79, no 1, p. 119-130, pl. 12.

Hoogenraad, H. R., 1934, Studien über die sphagnicolen Rhizopoden der niederländischen Fauna: D. Sc. thesis, University of Utrecht, p. I-IV +1-100, pls. 1-2, Lippert and Co. (Naumburg - Saale).

Hoogenraad, H. R., 1935a, Studien über die sphagnicolen Rhizopoden der niederländischen Fauna: Archiv für Protistenkunde, v. 84, no 1, p. 1-100, pls. 1-2.

Hoogenraad, H. R., 1935b, Chyphoderia laevis: Die levende Natuur, v. 40, p. (?)

Hoogenraad, H. R., 1936a, Zusammenstellung der fossilen Süsswasserrhizopoden aus postglazialen Sapropelium - und Torfablagerungen Europas: Archiv für

Protistenkunde, v. 87, no 3, p. 402-416.

Hoogenraad, H. R., 1936b, Was ist Pamphagus mutabilis Bailey?: Archiv für Protistenkunde, v. 87, no 3, p. 417-426.

Hoogenraad, H. R., de Groot, A. A., 1927, Rhizopoden en Heliozoën vit het zoetwater van Nederland. IV: Tijdschrift der Nederlansche Dierkundige Vereeniging, ser. 2, v. 20 , p. (?).

Hoogenraad, H. R., de Groot, A. A., 1935, Rhizopoden und Heliozoen aus dem Süsswasser der Niederlande. V: Nederlandsche Archieven Natuurkunde, v. 1, no 4, p. 432-488, figs. 1-16.

Hoogenraad, H. R., de Groot, A. A., 1937, Biometrische Untersuchungen an Süsswasserrhizopoden. (Rhizopoden und Heliozoen aus dem Süsswasser der Niederlande. VI): Archiv für Hydrobiologie, v. 31, p. 101-132, pls. 3.

Hoogenraad, H. R., de Groot, A. A., 1940a, Moosbewohnende thekamöbe Rhizopoden von Java und Sumatra: Treubia (Buitenzorg), v. 17, no4, p. 209-259.

Hoogenraad, H. R., de Groot, A. A., 1940b, Zoetwaterrhizopoden en heliozoën: in:.A.W.Sijthoff (ed?), Fauna von Nederland: Aflerering 9, p. 1-303, (Leiden).

Hoogenraad, H. R., de Groot, A. A., 1941, Observations on a special manner of feeding of a species of Difflugia (Difflugia rubescebns Penard). (Rhizopoda and Heliozoa of the Netherlands. VIII.): Proceedings, Proceedings of the Koninklijke Nederlandse Akademie van Wetenschappen, v. 44, no 2, p. 217-228, 2 pls.

Hoogenraad, H. R., de Groot, A. A., 1942, On fossil freshwater Rhizopods from tropical moors in Sumatra and Borneo: Proceedings of the Koninklijke Nederlandse Akademie van Wetenschappen, v. 45, no 7, p. 734-741.

Hoogenraad, H. R., de Groot, A. A., 1946, Thekamoebe sphagnumrhizopoden van Buitenzorg (Java): Biologische Jaarboek, Dodonea (Gent), v. 13, p. 112-126. 
Hoogenraad, H. R., de Groot, A. A., 1948, Thecamoebous moss-rhizopods from New Zealand: Hydrobiologia, v. 1, p. 28-43.

Hoogenraad, H. R., de Groot, A. A., 1952a, Thekamöbe Moorrhizopoden aus Nordamerika: Archiv für Hydrobiologie, v. 47, no 2, p. 229-262.

Hoogenraad, H. R., de Groot, A. A., 1952b, Thekamöbe Moorrhizopoden aus Sien: Archiv für Hydrobiologie, v. 47, no 2, p. 263-287.

Hoogenraad, H. R., de Groot, A. A., 1953, Über rotselhafte Elemente in de Schäle der thekamöben Moosrhizopoden Bullinula indica Penard: Archiv für Hydrobiologie, v. 47,.p. 1-8.

Hoogenraad, H.R., de Groot, A.A., 1979, Comparison of rhizopod associations: Hydrobiol.-Bull., v. 13, pp. 50-55

Hopkinson, J., 1910, The freshwater Rhizopoda and Heliozoa of County Wocklow: Irish Naturalist, v. 99, p. 1-4.

Hopkinson, J., 1911, Protozoa: in: St. Albans and its neighbourhood: Transactions of the Hertsfordshire Natural History Society, v. 14, no 3, p. 232.

Hopkinson, J., 1914, Euglypha alveolata or acanthophora: a problem in nomenclature: Zoologischer Anzeiger, v. 44, p. 526-528.

Hopkinson, J., 1919, Bibliography: in: Cash, J., Wailes, G. H. and Hopkinson, J., The British freshwater Rhizopoda and Heliozoa: v. 4, p. 72-122; Ray Society Publication no 103 (London).

Hoppman, J., 1954, Die Thekamoeben der Tormoose des venner Moores: Nature und Heimat, v. 14, p. 1-8.

Huber, G., 1907, Der Kaltersee (Südtirol): Archiv für Hydrobiologie, v. 2, no 4, p. 448464.

Huddleston, R. W., Haman, D., 1985, Mississippiellidae, a new Eulobosinid (Thecamoebinid) Family (Protozoa): Biological Society of Washington, Proceedings, v. 98, no 1, p. 10-12.

Hunt, G.W., Chein, S.M., 1983, Seasonal distribution, composition and abundance of the planktonic Ciliata and Testacea of Cayuga Lake: Hydrobiologia, v. 98, pp. 257-266

Hutchinson, G. E., 1967, A treatise on limnology. Volume II: Introduction to lake biology and the limnoplankton: 1115p, Wiley and Dons (New York).

Imhof, O. E., 1884, Weitere Mittheilung über die pelagische Fauna der Susswasserbecken: Zoologischer Anzeiger, v. 7, p. 321-327.

Imhof , O. E., 1885a, Notiz bezüglich der Difflugia cratera Leidy: Zoologischer 
Anzeiger, v. 8, p. 293-294.

Imhof, O. E., 1885b, Zoologische Mittheilungen: Viertelj. natur. Ges. Zürich, v. 30, p. 369-389.

Imhof, O. E., 1887a, Studien über die Fauna hochalpiner Seen insbesonder des Kantons Graubünden: Jahresbericht natur. Ges. Graubünden, v. 30, p. 145-164.

Imhof, O. E., 1887b, -title(?)- :Jahresbericht natur. Ges. Graubünden, v. 30, p. (?)

Imhof, O. E., 1888, Beitrag zur Kenntnis der Susswasserfauna der Vogesen: Zoologischer Anzeiger, v. II, p. 565-566.

Imhof, O. E., 1893, Les organismes inferieurs des lacs de la region du Rhone: Archives des Sciences Physique et Naturelles, v. 30, p. 646-652.

Ishii, K., Kano, F., 1973, An analysis of ectoplasmic movement in amoeba: in: Puytorac, P. de, and Grain, J.(eds.): Progress in Protozoology. Abstracts of papers read at the Fourth International Congress on Protozoology, Clermont-Ferrand, 2-9 September 1973: p.192, Université de Clermont, UER Sciences Exactes et Naturelles (Clermont-Ferrand).

Issel, R., 1901a, Saggio sulla fauna termale Italiana. Nota I.: Atti della Reale Accademia della Scienze di Torino, v. 36, p. 53-74.

Issel, R., 1901b, Saggio sulla fauna termale Italiana. Nota II.: Atti della Reale Accademia della Scienze di Torino, v. 36, p. 265-277.

Issel, R., 1901c, Osservazioni sopra alcuni animali della fauna termale italiana: Atti della Società Linguistica di Scienze naturali e geografiche, v. 12, no 1, p. 59-73, pls. 1-2.

Ivanega, I. G., 1975, title (?): Vestnik Zoologii (Kiev), v. for 1975, no 6, p. (?)

Ivanic, M., 1925, Über die multiple und jugendliche Teilung bei Centropyxis aculeata Ehrbg.: Zoologischer Anzeiger, v. 63, p. 267-(?).

Ivanic, M., 1934, Über die gewöhnliche Zweiteilung, multiple Teilung und Encystierung bei zwei Euglypha-Arten: Archiv für Protistenkunde, v. 82, no 3, p. 363-379, pls. 9-10.

Ivanic, M., 1935a, Über die volkommene "Kopulation" bei Cochliopodium digitatum Pénard, nebst Bemerkungen über die Kopulation bei Süsswasserthalamophoren im allgemeinen: Biologisches Zentralblatt, v. 55, no 5, 6, p. 225-245.

Ivanic, M., 1935b, Über die mit der "volkommenen Kopulation" verbundene multiple Teilung in Form einer Vierteilung bei Euglypha binucleolus sp. n.: Zoologischer Anzeiger, v. 110, p. 71-76.

Ivanic, M., 1935c, Nahrungsaufnahme mittels Pseudopodien und scheinharer 
Parasitismus bei einer Süsswasserthalamophore (Cochliopodium spec.): Zoologischer Anzeiger, v. 112, p. 230-233.

Jawerowski, A., 1891, Przyczynek do znajomosci rozmnazania roznozek (Rhizopoda) slodkowodnych: Kosmos (Lemberg), v. 16, p. 281-296, pl. I.

Jawerowski, A., 1895, Neue Arten der Brunnenfauna von Krakau und Lemburg: Archiv für Naturgeschichte, v. 61, no 1, p. 319-345.

Jeanson, C., Couteaux, M.M, 1977, Microanalyse élémentaire de la théque de Thécamoebiens du genre Euglypha: Academie des Sciences, Paris; Comptes Rendus Hebdomadaires des Seances, sér. D, v. 284, no 19, p. 1895-1898, pls. 1-3.

Jeanson, C., Couteaux, M.M., 1976, Analyses élémentaires de la thèque de Thécamoebiens en microscopie à balayage: Journal of Protozoology, v. 23, no 4, p. $13 \mathrm{~A}$.

Jekkel, A., Bereczky, M. C., 1977, Zönologische Untersuchung der TorfmoosTestaceen: Annales Universitatis Scientiarum Budapestinensis de Rolando Eötvös Nominatae (Sectio biologica), v. 18-19, p. 197-204.

Jeliffe, S. E., 1893, A preliminary list of the microscopical Animalculi found in the Ridgewood water-supply: American Monthly Microscopical Journal, v. 14, p. 289290.

Jennings, H. S., 1901, A report of work on the Protozoa of Lake Erie, with especial reference to the laws of their movements: Bulletin U. S. Fishery Commission, v. 19, p. 105-114.

Jennings, H. S., 1916, Heredity, variation and the results of selection in the uniparental reproduction of Difflugia corona: Genetics, v. I, p. 407-534.

Jennings, H. S., 1929, Genetics of the Protozoa: Bibliographia Genetica, v.5, p.105330

Jennings, H. S., 1937, Formation, inheritance and variation of the teeth in Difflugia corona. A study of the morphogenic activities of rhizopod protoplasm: Journal of Experimental Zoology, v. 77, no 2, p. 287-336.

Jennings, H. S., 1941, Inheritance in Protozoa: in: Calkins, G. N. and Summers, F. M. (eds.), Protozoa in biological research, p.710-771, Columbia University Press (New York).

Jepps, M. W., 1926, Contribution to the study of Gromia oviformis Dujardin: Quarterly Journal of Microscopical Science, new ser., v. 70, no 280, p. 701-719, pls. 37-39.

Jiaji, W., 1977, Protozoa from some districts of Tibetan plateau: Acta Zoologica Sinica, v. 23, pp. 131-160 (in Chinese). 
Jikeli, C. F., 1884, Ueber die Copulation von Difflugia globulosa Duj.: Zoologischer Anzeiger, v. 7, p. 449-451.

Joblot, L., 1754, Observations d'histoire naturelle faites avec le microscope, sur an grand nombre d'insects, \& sur les animalcules qui se trouvent dans les liqueurs préparées, \& dans celles qui ne sont pas, .avec la description \& les usages des différens (sic) microscopes, .partie déjà publiée par feu M. Joblot, partie rédigée sur ses observations postérieures: Briasson (Paris); 2 volumes.

John, S.J., 1995, Microfaunal assemblages, their use in interpreting depositional environments within a transgressive valley-fill sequence: Abstract, Geological Society of America, v. 27, 6, pp. 28. ..

Jollos, V., 1921, Experimentelle Protistenstudien. I. Untersuchungen über Variabilität und Vererbung bei Infusorien: Archiv für Protistenkunde, v. 43, no 1-2, p. 1-222.

Jollos, V., 1924a, Untersuchungen über Variabilität und Vererbung bei Arcellen: Biologische Zentralblatt, v.44, p.194-208.

Jollos, V., 1924b, Untersuchungen über Variabilität und Vererbung bei Arcellen: Archiv für Protistenkunde, v.49, p.307-374

Jollos, V., 1934, Dauermodifikationen und Mutationen bei Protozoen: Archiv für Protistenkunde, v. 83, no 1, p. 197-219.

Jones, T. R., 1847, Polygastria: in: Todd, R. B. (ed.), The Cyclopaedia of Anatomy and Physiology, v. 4, part 1, p. 2-18: Longman, Brown, Green, Longmans and Roberts (London).

Joyon, L., Charret, R., 1962, Sur 1 'ultrastructure du Thécamoebien Hyalosphenia papilio (Leidy): Academie des Sciences, Paris; Comptes Rendus Hebdomadaires des Seances, v. 255, no 20, p. 2661-2663, 4 pls.

Jung, W., 1934, Beobachtungen an der Moor-Thekamöbe Bullinula indica PENARD: Abhandlungen des westfälischen Provinz Museum, v. 5, p. (?).

Jung, W., 1936a, Thekamöben eines Eggegebirgsmoores und zweier Moore im Hohen Venn: Annales de Protistologie, v. V, p. 83-123.

Jung, W., 1936b, Thekamöben ursprünglicher, lebender deutscher Hochmoore: Abhandlungen aus dem Landesmuseum der Provinz Westfalen, Museum für Naturkunde, 7 Jahrang, Heft 4, p. 1-87.

Jung, W., 1942a, Südchilenische Thekamöben (Aus dem südchilenischen Küstengebiet, Beitrag 10): Archiv für Protistenkunde, v. 95, no 3, p. 253-356.

Jung, W., 1942b, Illustrierte Thekamöben-Bestimmungstabellen. I. Die Systematik der Nebelinen: Archiv für Protistenkunde, v. 95, no 3, p.357-390. 
Jux, U., Moericke, V., 1965, Tytthodiscus suevicus Eisenack, eine Thekamoebe?: Palaeontographica, Abteilung B: Palaeophytologie, v. 115, Part 4-6, pp. 107-115.

Kaska, Z., Lebkowska, M., Rzechowska, E., 1981, Studies on the effect of industrial wastes on the Protozoa development in the activated sludge: in: Dryl, S., Kazubsky, S. L. and Ploszaj, J., (eds.), Progress in Protozoology. Abstracts of papers submitted to the VI International Congress of Protozoology, Warszawa, Poland, July 5-11, 1981): p.172.

Kemma, A., 1905, La biologie des eaux potables: Mémoires de la Société Malacologique de Begique, v. 39, p. 9-132.

Kent, W. S., 1880-81, A manual of the Infusoria: including a description of all known flagellate, ciliate, and tentaculiferous Protozoa, British and foreign, and an account of the organization and affinities of the sponges: v. 1 (1880-81), p. 1-472; D. Bogue (London).

Kepner, W. A., 1905, Paulinella chromatophora: Biological Bulletin, Marine Biology Laboratory, Woods Hole, Mass., v. 9, p. 128-129.

Khainsky. A., 1910. Untersuchungen über Arcellen: Archiv für Protistenkunde, v. 21, no 2, p. 165-185, pls. 14, 15.

Kikuchi, K., 1930, A comparison of the diurnal migration of plankton in eight Japanese lakes: Memoires of the College of Science, Kyoto Univ., ser. B, v. 5, p. 27-46.

Kliza, D.A., Schröder-Adams, C.J., 1994, Distribution of Arcellacea in freshwater lakes in Pond Inlet and Bylot Island, Northwest Territories: Abstract, Geological Society of America, v. 26, pp. 326.

Kliza, D.A., 1994, Distribution of Arcellacea in freshwater lakes of Pond Inlet and Bylot Island, Northwest Territories. Unpublished honours thesis, Carleton University, Ottawa, Ontario: 52 pp.

Kobylinska, D.(ed.), 1974, Katalog zbiorow geologicznych Instytutu Geologicznego, dzial paleontologiczno-stratygraficzny, fauna kopalna z obszaru Polski, Kenozoik [Catalog of geological collections in the Geological Institute, Paleontological-Stratigraphic Division, Fossil fauna from the territory of Poland, Cenozoic], Wydawn. Geol. 73p.

Koffman, M., 1934, Die Mikrofauna des Bodens,ihr Verhaltnis zu anderen Boden: Archiv für Mikrobiologie, v. 5, p. 246-302.

Kofoid, C. A., 1903, The Plankton of the Illinois River, 1894-1899. Part I. Quantitative investigations and general results: Bulletin of the Illinois State Laboratory, v. 6, no 2, p. 95-629.

Kofoid, C. A., 1908, The Plankton of the Illinois River, 1894-1899. Part II. Constituent 
organisms and their seasonal distribution: Bulletin of the Illinois State Laboratory, v. 8, no 1, p. 1-361.

Kofoid. C. A., 1923, The life cycle of the Protozoa: Science v. 57, p. 397-408.

Korganova, G. A., 1974, O vidovom sostave rakovinnkh kornenozhek (Testacida, Protozoa) v nekotorkh pochvakh Tambovskoi nizmennosti (On the species composition of the shelled Rhizopods (Testacea, Protozoa) in some soils of the Tambov depression): Dinamika mikrobiologicheskikh protsessov v pochve i obuslovlivayushchie ee faktor, part 2, p. 171-175; (Tallin).

Korganova, G. A., 1975, Rakovinne ameb (Testacida) nekotorkh pochv Evropeiskoi chastu SSSR (Testaceae of some soils of the European part of USSR): Pedobiologia, v. 15 , no 6 , p. $425-431$.

Korganova, G. A., 1976, Fauna rakovinnkh ameb v pochvakh el'nikov (Fauna of shelled amoebae in fir forest soils): in: Materiali 2-go Vsesoyuznogo s"ezda protozoologov", part 1, p.70-71; (Kiev).

Korganova, G. A., 1977, Adaptatsionne osobennosti pochvoobitayushchikh rakovinnkh ameb (Protozoa, Testacida) (Adaptation of Testacida to soil conditions: in: Gilyarov, M. S. (ed.), Adaptatsiya pochvennkh zhivotnkh k usloviyam sred, p. 82-101; Nauka (Moskva).

Korganova, G. A., 1978a, Testacida (Protozoa) as an indicator of soil conditions: Scientific Information (Soil Zoology), p. 153-156.

Korganova, G. A., 1978b, Testacida as an indicator of soil conditions in Moscow region forests: in: Problems of Soil Zoology, p. 116-118; (Minsk).

Korganova, G. A., 1981, Testacid distribution in forest soils: in: Dryl, S., Kazubski, S. L. and Ploszaj, J. (eds.): Progress in Protozoology. Abstracts of papers submitted to the VI International Congress of Protozoology, Warszawa, Poland, July 5-11, 1981, p.192; (Warszawa).

Korganova, G. A., Geltzer, J. G., 1975, Testacida in two types of soil in Leningrad region: in: Vanek, J., (ed.): Progress in soil zoology. Proceedings of the 5th International Colloquium on Soil Zoology held in Prague September 17-22, 1973, p. 121-124; Academia, Publishing House of the Czechoslovak Academy of Sciences (Prague).

Korganova, G. A., Geltzer, J. G., 1977, Stained smears for the study of soil Testacida (Protozoa, Rhizopoda): Pedobiologia, v. 17, no 3, p. 222-225.

Korotnef, A. A., 1880, Etudes sur les Rhizopodes: Archives de Zoologie Experimentale et Generale, v. 8, p. 467-482. 


\section{KOU-LON}

Kourov, 0., 1925, Faune Rhizopodique des bassins de Kossino: Trudy Kosinskoi Biologicheskoi Stantsii, v. 2, p. 43-68.

Kraschennikoff, S. A., 1922, Beobachtungen über die Verbreitung der Rhizopoden im See Glubokoje: Russ. Hydrobiologische Zeitschrift, v. I, p. I-8.

Krylov, M. V., Dobrovol'skii, A. A., Issi, I. V., Mikhalevich, V. I., Podlipaev, S. A., Reshetnyak, V. V., Seravin, L. N., Starobogatov, Y. I., Shul'man, S. S. And Yankovskii, A. V., 1980, Novye predstavleniya o sisteme odnokletochnykh zhivotnykh (New proposals for the system of the unicellular animals): in: Kyrlov, M. A. and Starobogatov, Y. I. (eds.), Printsipy postroeniya makrosistemy odnokletochnykh: Trudt Zoologicheskogo Instituta Akademii Nauk SSSR, v. 94, p. 122-132.

Kufferath, H., 1932, Rhizopodes du Congo: Revue de Zoologie et de Botanique Africaines. v. 23, no 1, p. 52-60, pls. 3, 4.

Kühn, A., 1926a, Morphologie der Tiere in Bildern. Heft 2: Protozoen. Teil 2. Rhizopoden: p. I-IV, 107-272; Bornträger (Berlin).

Kühn, A., 1926b, Grundriss der allgemeinen Zoologie. 2 Auflage: p.I-VIII, 1-261: Georg Thieme (Leipzig).

Kumar, A. and Dalby, A.P., 1998. Identification Key for Holocene Lacustrine Arcellacea (Thecamoebian) Taxa. Palaeontologia Electronica, 1(1): 36p., 3.1 MB. http://www-odp.tamu.edu/paleo/1998 1/dalby/issue1.htm

Kunhelt, W., 1955a, An introduction to the study of soil animals: in: Kevan, K. Mc. (ed.): Soil zoology. Proceedings of the University of Nottingham Second Easter School on Agricoltural Science: p. 3-22, Butterworths Scientific Publications (London).

Kunhelt, W., 1955b, A brief introduction to the major groups of soil animals and their biology: in: Kevan, K. Mc. (ed.): Soil zoology. Proceedings of the University of Nottingham Second Easter School on Agricoltural Science: p. 29-43, Butterworths Scientific Publications (London).

Kvry, J., 1956, Thékambák (Testaceák) a majyarországi alsópannóniai korú üledékekbl: Földtani Közlöny, v. 86, no 3, p. 266-272, pls. 35-39.

Labbé, A., 1895, Les théories récentes sur l'homologation du noyau des Protozoaires et du noyau des cellules des Métazoaires: Archives de Zoologie expérimental et générale, v. 3, no.3, p. 10-14.

Lachmann, J., 1859, Über Rhizopoden-Infusorien der Gegend von Bonn:

Verhandlungen des naturwissenshaftlichen Vereins Rheinlande, v. 16 (Supplement: Sitzsungsberichte), p. 57-93. 
Lagerheim, G., 1901, Om lämningar af Rhizopoder, Heliozoer och Tintinnider i Sveriges och Finlands lakustrina kvartäraflagringar: Geologiska Föreningen i Stockholm Förhandlingar, v. 23, no 2, p. 469-520.

Lagerheim, G., 1902, Om Quadrula subglobosa Lagerheim: Geologiska Föreningen i Stockholm Förhandlingar, v. 24, p. 346-352.

Lamarck, J.B., 1816, Histoire naturella des animaux sans vertèbres: Tone 2, p. 1-568, Verdière (Paris).

Lambert, J., Chardez, , D., 1978, Intérêt criminalistique de la microfaune terrestre: Revue Internationale de Police criminelle, no 319, p. 158-170.

Lambert, J., Chardez, D., 1976, Note sur les Thécamoebiens du sol au Zaïre: Revue Verviétoise d'Histoire Naturelle, v. 33, no 10-12, p. 61-63.

Laminger, H., 1971, Ein Beitrag zur Kenntnis der Thekamoebenfauna Oesterreichs (Protozoa, Rhizopoda testacea): Zoologischer Anzeiger, v. 187, pp. 372-382.

Laminger, H., 1971, Sedimentbewohnende Schalenamöben (Rhizopoda Testacea) der Finstertaler Seen (Tirol): Archiv für Hydrobiologie, v.69, no 1, p. 106-140.

Laminger, H., 1972a, Die profundale Testaceenfauna (Protozoa Rhizopoda) älterer und jüngerer Bodensee-Sedimente: Archiv für Hydrobiologie, v. 70, no 1, p. 108-129.

Laminger, H., 1972b, Ein beitrag zur Kenntnis der Hochgebirgs-Testaceen Österreiche: Archiv für Protistenkunde, v. 114, no 1-2, p. 101-151.

Laminger, H., 1972c, Notes on some terrestrial Testacea (Protozoa Rhizopoda) from Nepal, Himalaya (Lhotse Shar): Archiv für Protistenkunde, v. 114, no 4, p. 486-488.

Laminger, H., 1972d, Terrestrische Testaceen (Protozoa Rhizopoda) in der Omgebung von Obergurgl (Österreiche, Tirol): Pedobiologia, v. 12, p. 16-22.

Laminger, H., 1973, Untersuchung ueber die Testaceenfauna (Protozoa, Rhizopoda) in den juengsten Bodensee-Sedimenten: Biologische Jaarboek., v. 41, pp. 126-146.

Laminger, H., 1973a, Facteurs responsables de la distribution saisonnière et spatiale des Thécamoebiens d'un sédiment d'un lac de haute montagne: in: DePuytorac, P. and Grain, J. (eds.): Progress in Protozoology. Abstracts of papers read at the Fourth International Congress on Protozoology, Clermont-Ferrand, 2-10 Sept. 1973): p.239; Université de Clermont (Clermont-Ferrand).

Laminger, H., 1973b, Die Testaceen (Protozoa, Rhizopoda) einiger Hochgebirgsgewässer von Mexiko, Costa Rica und Guatemala: International Revue der Gesamten Hydrobiologie, v. 58, no 2, p. 273-305.

Laminger, H., 1973c, Die Testaceenfauna (Protozoa Rhizopoda) in der Umgebung von Obertavern (Salzburg): Archiv für Protistenkunde, v. 115, no 2-3, p. 253-270. 
Laminger, H., 1973d, Untersuchung über Abundanz und Biomasse der sediment bewohnenden Testaceen (Protozoa Rhizopoda) in einem Hochgebirgssee (Vorderer Finstertaler See, Kuthai, Tirol): International Revue der Gesamten Hydrobiologie, v. 58, no 4, p. 543568.

Laminger, H., 1973e, Zur kenntnis der Testaceenfauna in den jungsten Sedimenten des Bodenseeprofundals und litorals: Schweizerische Zeitschrift für Hydrologie, v. 35 , no 2, p. 239-246.

Laminger, H., 1973f, Quantitative Untersuchung über die Testaceenfauna (Protozoa Rhizopoda) in den jungsten Bodensee-Sedimenten: Biologische Jaarboek, v. 41, p. 126-146.

Laminger, H., 1974, Ein Beitrag zur Kenntnis der Protozoenfauna der Donau. I. Die Testaceen (Protozoa Rhizopoda) im Sbschnitt Obernzell Linz (Oberösterreich): Archiv für Hydrobiologie, v. 44, no 3, p. 330-337.

Laminger, H., 1975, Die Sukzession der Testaceen-Assoziationen (Protozoa Rhizopoda) im rezenten und subfossilen Sphagnum des Obersees bei Lunz (Niederösterreich): Hydrobiologia, v. 46, fasc. 4, p. 465-487.

Laminger, H., 1975, Die Sukzession der Testaceen-Assoziationen (Protozoa, Rhizopoda) im rezenten und subfossilen Spagnum des Obersees bei Lunz (Niederoesterreich): Hydrobiologia, v. 46, pp. 465-487.

Laminger, H., 1975, Die Sukzession der Testaceen-Assoziationen (Protozoa, Rhizopoda) im rezenten und subfossilen Spagnum des Obersees bei Lunz (Niederoesterreich): Hydrobiologia, v. 46, pp. 465-487.

Laminger, H., 1978, The effects of soil moisture fluctuations on the testacean species Trinema enchekys (Ehrenberg) Leidy in a high mountain brown-earths-podsol and its feeding behaviour: Aechiv für Protistenkunde, v. 20, no., p. 446-454.

Laminger, H., 1980, Bodenprotozoologie: Mikrobios, v. 1, p. 1-142 (Innsbruck).

Laminger, H., Geisler-Moroder, K., Siess, A., Spiss, E., Spiss, B., 1980, Populationsdynamik terrestrischer Protozoa (Testacea Rhizopoda) in zentralalpinen Lagen Tirols. I. Untersuchungen subalpiner Böden im Raum Obergurgl (Tirol/Österreich): Archiv für Protistenkunde, v. 123, no 3, p. 280-323.

Laminger, H., Schopper, M., Pipp, E., Hensler, I., Mantl, P., 1981, Untersuchungen über Nekrozönosen und Taxozönosen der Testacea (Protozoa) im Zirbenwaldmoor (Obergurgl, Tirol/Austria): Hydrobiologia, v. 77, p. 193-202.

Laminger, H., Zisette, R., Phillips, S., Breidigam, F., 1979, Beitrag zur Kenntnis der Protozoenfauna Montanas (U.S.A.): I. Die Testaceen (Rhizopoda) in der Region des Flathead-Lake-Tales: Hydrobiologia, v. 65, no 3, p. 257-271. 
Landacre, F. L., 1908, The protozoa of Sandusky Bay and its vicinity: Proceedings of the Ohio Academy of Science, v. 4, no 10, p. 427-429.

Lanessan, J. L. de, 1880, Les Protozoaires: Revue Internationale des Sciences, v. 6 , p. 1-49.

Lanessan, J. L. de, 1882, Traité de zoologie. I. Les Protozoaires: Octave Doin, Paris; $336 \mathrm{p}$.

Lang, A., 1888-1889, Lerbuch der vergleichenden Anatomie zum Gebrauche bei vergleichend anatomischen und zoologischen Vorlesungen: Band 1, p.i-xvi + 1290. [translated in Lang 1882].

Lang, A., 1891, Text-book of comparative anatomy. Vol. I: McMillan and Co., London; p.1-566.

Lang, A., 1892, Traité d'anatomie compare et de zoologie. Vol. I. G.Carro and C.Naud, Paris; p. (???).

Lang, A., 1892-1894, Lehrbuch der vergleichenden Anatomie der Wirbellosen Thiere: Band II, p.i-vi + 1-311; Gustav Fischer (Jena). [translated in Lang 1891].

Lang, F. H., 1865, A new Difflugia: Quarterly Journal of microscopical Science, new ser., v. 5, p. 285-286.

Lauterborn, R., 1895a, Protozoen-Studien. II. Paulinella chromatophora nov. gen. nov. spec., ein beschalter Rhizopode des Süsswasser mit blaugrünen chromatophorenartiger Einschlüssen: Zeitschrift für wissenschaftlichen Zoologie, v. 59, no 4, p. 534-544, p1. 30.

Lauterborn, R., 1895b, title (?): Journal of the Royal Microscopical Society, p. 542543.

Lauterborn, R., 1901, Die "sapropelische" Lebewelt: Zoologischer Anzeiger, v. 24, p. 50-55.

Lauterborn, R., 1908, Protozoen Studien. V. Zur Kenntniss einiger Rhizopoden und Infusorien aus dem Gebiete des Oberrheins: Zeitschrift für Wissenschaftliche Zoologie, v. 90, p. 645-669.

Laybourn, J., Whymant, L., 1980, The effect of diet and temperature on reproductive rate in Arcella vulgaris Ehrenberg (Sarcodina, Testacida): Oecologia, v. 45, p. 282-284.

Leclerc, L., 1816, Note sur la Difflugie, nouveau genre de Polype amorph: Mémoires du Muséum d'Histoire Naturelle (Paris) v. 2, no 12, p. 474-478, p1. 17.

Leclerc, L., 1817, Über die Difflugia, neue Sippe von ungestaltigen Polypen (with comments by L. von Oken): Isis (oder encyclopädische Zeitung), Zrich, v. I, columns 980-984, p1. 7. 
Leidy, J., 1874a, Remarks on Protozoa: Proceedings of the Academy of Natural Sciences of Philadelphia, 3rd ser., v. 26, p. 13-15.

Leidy, J., 1874b, Notice of some new fresh-water Rhizopods: Proceedings of the Academy of Natural Sciences of Philadelphia, 3rd ser., v. 26, p. 77-79.

Leidy, J., 1874c, Notice of some Rhizopods: Proceedings of the Academy of Natural Sciences of Philadelphia, 3rd ser., v. 26,p. 155-157.

Leidy, J., 1874d, Note on the enemies of Difflugia: Proceedings of the Academy of Natural Sciences of Philadelphia, 3rd ser., v. 26, p. 75.

Leidy, J., 1874e, Notice of some fresh-water and terrestrial rhizopods: Proceedings of the Academy of Natural Sciences of Philadelphia, 3rd ser., v. 26, p. 86-88.

Leidy, J., 1875a, Notices of rhizopods: Proceedings of the Academy of Natural Sciences of Philadelphia, 3rd ser., v. 26, p. 225-227 (1874).

Leidy, J., 1875b, Remarks on rhizopods: Proceedings of the Academy of Natural Sciences of Philadelphia, 3rd ser., v. 27, p. 413-415.

Leidy, J., 1876a, Remarks on Arcella etc.: Proceedings of the Academy of Natural Sciences of Philadelphia, 3rd ser., v. 28, p. 54-58..

Leidy, J., 1876b, Remarks on the rhizopod genus Nebela: Proceedings of the Academy of Natural Sciences of Philadelphia, 3rd ser., v. 28, p. 115-119.

Leidy, J., 1876c, Observations on rhizopods: Proceedings of the Academy of Natural Sciences of Philadelphia, 3rd ser., v. 28, p. 197-199.

Leidy, J., 1877a, The birth of a rhizopod: Proceedings of the Academy of Natural Sciences of Philadelphia, 3rd ser., v. 29, p. 261-265.

Leidy, J., 1877b, Remarks upon rhizopods, and notice of a new form: Proceedings of the Academy of Natural Sciences of Philadelphia, 3rd ser., v. 29, p. 293-294.

Leidy, J., 1878a, Remarks upon the American species of Difflucia: Proceedings of the Academy of Natural Sciences of Philadelphia, 3rd ser., v. 29, p. 306-308 (1877).

Leidy, J., 1878b, Rhizopods in an apple-tree: Proceedings of the Academy of Natural Sciences of Philadelphia, 3rd ser., v. 29, p. 321 (1877).

Leidy, J., 1878c, Species of Euglypha, Trinema, Pamphagus, and Cyphoderia, with synonyms and descriptions of new forms: Proceedings of the Academy of Natural Sciences of Philadelphia, 3rd ser., v. 30, p. 171-173 (1878).

Leidy, J., 1879a, Fresh-water Rhizopods of North America: Report of the United States Geological Survey of the Territories, v. 12, p. i-xi + 1-324, pl. 1-48. 
Leidy, J., 1879b, On rhizopods occurring in Sphagnum: Proceedings of the Academy of Natural Sciences of Philadelphia, 3rd ser., v. 31, p. 162-163.

Leidy, J., 1880a, Fresh-water rhizopods of North America: American Journal of Science, 3rd ser., v. 19, p. 240-244.

Leidy, J., 1880b, Rhizopods in the mosses on the summit of Roan Mountain, North Carolina: Proceedings of the Academy of Natural Sciences of Philadelphia, 3rd ser., v. 32, p. 333-340.

Lemmerman, E., 1907, Das plankton des Jang-tse-Kiang (China): Archiv für Hydrobiologie, v. 2, no 4, p. 534-544.

Lena, H., 1982, Benthic testacida (Rhizopoda, Protozoa) of Lake Washington, Brevard County, Florida: Florida-Science, v. 45, pp. 101-106.

Lena, H., 1982, Cysts of Difflugia mitriformis (Testacea Lobosia) with external characteristics of Psammosphaera (Foraminifera): Revista Española de Micropaleontologia, v. 14, no 1-3, p. 151-152, 1 pl.

Lena, H., 1983, On the taxonomic validity of two testaceans, Protozoa, Rhizopoda, Difflugia pyriformis Perty, forma compressa Carter and Pontigulasia compressa Rhumbler: Revista Española de Micropaleontologia, v. 15, pp. 409-414.

Lena, H., 1983, Testaceolobosia (Protozoa Rhizopoda) of Melbourne, Florida, USA: Revista Española de Micropaleontologìa, v. 15, no 2, p. 317-328, pls. 1-3

Lena, H., 1984, Revision de las Tecamebas (Rhizopoda Protozoa) citadas en Publicaciones foraminiferologicas: Revista Española de Micropaleontologìa, v. 16, no 16 , p. 5-18.

Lena, H., Cachi, J.C., 1972, Tecamebas de la Laguna de Chascomús (Buenos Aires, Argentina): Revista Española de Micropaleontologìa, v. 4, no 3, p. 377-386, pl. 1.

Lena, H., de Zaidenwerg, S.J., 1975, Tecamebas del Delta del Parana (Argentina): In: Numero especial dedicado a la micropaleontologia de America del Sur: Perconig, E., Bermudez, P.J., Martinez-Diaz, C., Moreno-de-Castro, E., Granados-Luis, F.(eds.), Revista Española de Micropaleontologia, v. 7, no. 3, p. 519-537, pls. 1-6.

Lendenfeld, R. von, 1886, The Australian fresh-water Rhizopods. Part I: Proceedings of the Linnean Society of New South Wales, v. 10, p.723-725 (1855?).

Lepinis, A. K., Gel'tser, Y. G., Chibisova, O. I., Geptner, A. V., 1973, Opredelitel' Protozoa pochv Evropeiskoi chasti SSSR (Identification key for the soil Protozoa of the European part of the USSR): Mintis (Vil'nius), p. 1-172.

Levander, K. M., 1893, Verzeichnis der wärhend des Sommers 1891 bei Rostock beobachteten Protozoen: Archiv des Vereins der Freunde der Naturgeschichte in 
Mecklenburg, v. 46, p. 113-118.

Levander, K. M., 1894a, Materialen zur Kenntnis der Wasserfauna in der Umgebung von Helsingfors, mit besonderer Berücksichtigung der Meeresfauna. I. Protozoen: Acta Societatis pro Fauna et Flora Fennica, v. 12, no 2, p. 1-115, pls. 1-3.

Levander, K. M., 1894b, Liste über im Finnischen Meerbusen in der Umgebung von Helsingfors beobachteten Protozoen: Zoologischer Anzeiger, v. 17, p. 209-212.

Levander, K. M., 1900a, Zur Kenntnis des Lebens in den stehenden Kleingewässern auf den Skäreninseln: Acta Societatis pro Fauna et Flora Fennica, v. 18, no 6, p. 1107.

Levander, K. M., 1900b, Zur Kenntnis der Fauna und Flora finnischer Binnenseen: Acta Societatis pro Fauna et Flora Fennica, v. 19, no 2, p. 1-55.

Levander, K. M., 1901a, Zur Kenntnis des Planktons und der Bodenfauna: Acta Societatis pro Fauna et Flora Fennica, v. 20, no 5, p. 1-34.

Levander, K. M., 1901b, Übersicht in der Umgebung von Esbo-Lofo im Meereswässer vorkommenden Thiere: Acta Societatis pro Fauna et Flora Fennica, v. 20, no 6, p. 1 20.

Levander, K. M., 1901c, Beiträge zur Fauna und Algenflora der süssen Gewässer an der Murmanküste: Acta Societatis pro Fauna et Flora Fennica, v. 20, no 8, p. 1-35.

Levander, K. M., 1902, Meddelande om Paulinella chromatophora: Meddelande Societatis pro Fauna Fennica, v. 28, p. 26-27.

Levander, K. M., 1905, Zur Kenntniss des Planktons einiger Binne seen in RussischLapland: Festschrift für Palmen, v.1, no 2, p. 1-49, 3 pls.

Levine, N. D., Corliss, J. 0., Cox, F. E. G., Deroux, G., Grain, J., Honiger, B. M., Leedale, G. F., Loeblich, A. R., lii, Lom, J., Lynn, D., Merinfeld, E. G., Page, F. C., Poljansky, G., Sprague, V., Vavra, J., Wallace, F. G., 1980, Newly revised classification of the Protozoa: Journal of Protozoology, v. 27, no. 1, p. 37-58.

Lister, J. J., 1903, The Protozoa: Section I. The Foraminifera in: Lankaster, E.R. (ed.): A Treatise on Zoology; Part I: Introduction and Protozoa, Fasc. 2, p. 47-149.

Lloyd, F. E., 1929, The behavior of Vampyrella lateritia with special reference to the work of Prof. Chr. Gobi: Archiv für Protistenkunde, v. 67, p. 219.

Loeblich, A. R. Jr., Tappan, H., 1961a, Remarks on the systematics of the Sarcodina (Protozoa), renamed homonyms and new and validated genera: Proceedings of the Biological Society of Washington, v. 74, p. 213-234.

Loeblich, A. R. Jr., Tappan, H., 1961b, Suprageneric classification of the Rhizopoda: Journal of Paleontology, v. 35, no 2, p. 245-330. 
Loeblich, A. R. Jr., Tappan, H., 1964, Sarcodina, chiefly "Thecamoebians" and Foraminiferida: in: Moore, R. C. (ed.): Treatise on Invertebrate Paleontology: Part C, Protista 2, v. I, p.i-xxxi + 1-510a(16-54): Geological Society of America and University of Kansas Press.

Longhi, P., 1892, Protisti delle acque dolci di Genova e dintorni: Atti della Società Ligustica di Scienze naturali e geografiche, v. 3, p. 137-161.

Longhi, P., 1894, Prime ricerche intorno ai Protisti del distretto di Belluno e suoi dintorni: Atti della Società Ligustica di Scienze naturali e geografiche, v. 5, p. 1327.

Longhi, P., 1895, Seconda serie di ricerche intorno ai Protisti delle acque dolci del distretto di Belluno e suoi dintorni: Atti della Società Ligustica di Scienze naturali e geografiche, v. 6, p. 67-83, pls. 3. 


\section{LOR-NUT}

Lord, J. E., 1891a, Rosendale Rhizopods: Science Gossip, v. 27, p. 267-268.

Lord, J. E., 1891b, Rosendale Rhizopods: Science Gossip, v. 27, p. 276.

Lord, J. E., 1892a, Rosendale Rhizopods: Science Gossip, v. 28, p. 129-130.

Lord, J. E., 1892b, Fresh-water Rhizopods found in the neighborhood of Rawtenstall:

Transactions of the Manchester Microscopical Society, v. for the year 1891, p. 5557.

Lord, J. E., 1904, On a peculiar habit of Pamphagus hyalinus: Transactions of the Manchester Microscopical Society, v. for the year 1903, p. 76-78, pl. 3.

Lord, J. E., 1905, The micro-flora and fauna of our wells and surface throughs:

Transactions of the Manchester Microscopical Society, v. for the year 1904, p. 5557.

Lousier, J. D., 1974a, Effects of experimental soil moisture fluctuations on turnover rates of Testacea: Soil Biology and Biochemistry, v. 6, p. 19-26.

Lousier, J. D., 1974b, Response of soil Testacea to soil moisture fluctuations: Soil Biology and Biochemistry, v. 6, p. 235-239.

Lousier, J. D., 1975, Relationships between distribution of Testacea (Protozoa Rhizopoda) and the soil habitat: Naturaliste Canadien, v. 102, no 1, p. 57-72.

Lousier, J. D., 1976, Testate Amoebae (Rhizopoda Testacea) in some Canadian Rocky Mountain soils: Archiv für Protistenkunde, v. 118, p. 191-201.

Lousier, J. D., Elliot, M. J., 1975, Colonization of deciduous leaf litter by testate amoebae (Rhizopoda Testacea) and bacteria in: Kilbertus, G., Reisinger, 0., Mourey, A. and Cancela da Fonseca, J. A.(eds.): "Biodgradation et humification.": p. 98-107; Pierron, Sarreguemines (France).

Lousier, J. D., Parkinson, D, 1981b, Evaluation of a membrane filter technique to count soil and litter Testacea: Soil Biology and Biochemistry, v. 13, p. 209-213.

Lousier, J. D., Parkinson, D., 1981a, The disappearance of the empty tests of litterand soil-testate amoebae (Testacea Rhizopoda Protozoa): Archiv für Protistenkunde, v. 124, p. 312-336.

Lowman, S. W., 1949, Sedimentary facies in Gulf Coast: Bulletin of the American Association of Petroleum Geologists, v. 33, no 12, p. 1939-1997.

Lüftenegger, G., Petz, W., Berger, H. Foissner, W., Adam, H. (1988) Morphological and biometric characterisation of twenty-four testate amoebae. Archiv für 
Protistenkunde, 124 312-336.

Lwoff, A., 1925, La sporogenèse chez une Gromie marine: Gromia dujardini: Travaux de la Station zoologique de Wimereux, v. 9, p. 141-145.

Mackinlay, R. B., 1936, Observations on Nebela collaris Leidy (pro-parte), a testate amoeba of moorland waters: Part I: Journal of the Royal Microscopical Society, ser. 3 , v. 56 , p. $307-325$, pls. $1-2$.

Maes, G., Paulus, J., Chardez, D., 1972, Thécamoebiens d'un sol forestier zaïrois: Bulletin des Recherches Agronomiques de Gembloux, new sér., v. 7, no 1-2-3-4, p. 230-232.

Maggi, L., 1876, Intorno ai Rizopodi d'acqua dolce della Lombardia, ed in particolare del "Podostoma filigerum Clap. e Lach.": Rendiconti del Reale Istituto Lombardo di Scienze e Lettere, ser. 2, v. 9, p. 538-550, pl. 4.

Maggi, L., 1877a, Contribuzione alla morfologia delle Amphizonella: Rendiconti del Reale Istituto Lombardo di Scienze e Lettere, ser. 2, v. 10, p. 315-323, pl. 2.

Maggi, L., 1877b, Sull'esistenza dei Moneri in Italia: Rendiconti del Reale Istituto Lombardo di Scienze e Lettere, ser. 2, v. 10, p. 360-371.

Maggi, L., 1877c, Contribuzione al catalogo dei Rizopodi d'acqua dolce della Lombardia e loro distribuzione secondo la classificazione di Hertwig e Lesser modificata da Archer: Atti della Società Italiana di Scienze Naturali, v. 20, p. 313-319.

Maggi, L., 1880, Esame protistologico delle acque di alcuni laghi Italiani: Bollettino Scientifico (Pavia), v. 1, anno 2, no 2, p. 33-43.

Maggi, L., 1888a, Intorno ai Protozoi viventi sui muschi delle piante: Rendiconti del Reale Instituto Lombardo di Scienze e Lettere, ser. 2, v. 21, p. 300-311. [translated in Maggi 1888b].

Maggi, L., 1888b, Sur les Protozoaires vivant sur les mousses des plantes: Archivio Italiano di Biologia, v. 10, no 2, 184-189.

Maggi, L., 1893, Alcuni nuovi Protisti: Bollettino Scientifico (Pavia), v. 4, anno 15, no 1, p. 13-17.

Malhotra, Y. R., Dutta, S. P. S., 1977, On some freshwater Rhizopoda from Jammu: Proceedings of the Indian Science Congress, v. 64, p. 217.

Margalef, R., 1946, Algunos rizopodos testáceos de Cataluña: Boletin de la Real Sociedad Española de Historia Natural, v. 44, no 1-2, p. 73-75.

Margalef, R., 1955, Contribución al estudio de la fauna de las aguas dulces del noreste de España: Publicacione del Instituto de Biologia aplicada de Barcelona, v. 21, p. 137-171. 
Marsson, M., 1901, Zur kenntnis der Planktonverhaltnis einiger Gewasser der Umgebung von Berlin: Forschungsberichte aus der Biologischen Station zu PIon, v. 8, p. 86-119.

Matic, Z., Bunescu, V., 1977, Aspects concernant la densité et la biomasse des thécamoebiens (Protozoa: Rhizopoda, Testacea) des sols des Monts Bucegi Roumanie: Pedobiologia, v. 17, no 5, p. 297-304.

Maupas, E., 1882a, Sur le Lieberkuehnia, Rhizopode d'eau douce multinucléé: Comptes rendus des Séances de l'Académie des Science, Paris, v. 95, p. 191-194. [translated in Maupas, 1882b].

Maupas, E., 1882b, On Lieberkuehnia, a freshwater multinucleated Rhizopod: Annals and Magazine of Natural History, ser. 5, v. 10, p. 410-413.

McCarthy, F.M.G., Kerr, H.A., Scott, D.B., Collins, E.S., McAndrews, J.H., 1987, Arcellaceans as paleoecological indicators in Atlantic Canada: Abstract, Congress of the International Union for Quaternary Research, pp. 223.

McCarthy, F.M.G., Collins, E.S., MCAndrews, J.H., Kerr, H.A., Scott, D.B., Medioli, F.S. (1995) A comparison of postglacial arcellacean ("Thecamoebian") and pollen succession in Atlantic Canada, illustrating the potential of arcellaceans for paleoclimatic reconstruction. Journal of Paleontology, 69 980-993.

McCourt, R.M., Reshly, E., 1993, Spirogyra filament morphology affects predation by the protozoan Difflugia: Journal of Phycology, v. 29, suppl. no 3, p. 13.

McMurrich, J. P., 1883, Notes on some Canadian Infusoria: Proceedings of the Royal Canadian Institute, new ser., v. 1, fasc. 4, p.300-309, pl. I (plate published in v. 1, fasc. 5).

Medioli, B.E., 1995, Marginal marine foraminifera and thecamoebians in the Upper Cretaceous to Eocene deposits of the south-central Pyrenees, Spain: In: Atlantic Universities Geological Conference 1995, abstraacts, Atlantic Geology, v. 31, 3, p. 211.

Medioli, B.E., 1995, Marginal marine foraminifera and thecamoebians in the Upper Cretaceous to Eocene deposits of the south-central Pyrenees, Spain: Unpublished B. Sc. Honors Thesis, Dalhousie University, Halifax, N.S., Canada.

Medioli, F. S., Scott, D. B., 1983, Holocene Arcellacea (Thecamoebians) from Eastern Canada: Cushman Foundation for Foraminiferal Research, Special Publication no. 21, p. 1-63, pls. 1-7.

Medioli, F.S., Scott, D. B., 1985, Designation of types, for one genus and nine species of Arcellaceans (Thecamoebians) with additional reference material for four other species: Journal of Foraminiferal Research, v. 15, no 1, p. 24-37, pl. 1. 
Medioli, F.S., Asioli, A. Parenti, G., 1994, Manuale per l'identificazione e la classificazione delle tecamebe con informazioni sul loro significato paleoecologico e stratigrafico: Paleopelagos, v.4, p. 317-364.

Medioli, F.S., Petrucci, F., Scott, D.B., 1985, Sulla presenza di Tecamebe in Campioni Carotati sul Fondo del Lago di Garda "Missione Piccard - Settembre 1981": L’Ambiente Lago, Comunità del Garda, p. 83-103, 2 pls.

Medioli, F.S., Scott, D.B., 1988a, Lacustrine thecamoebians (mainly Arcellaceans) as potential tools for palaeolimnological interpretations: Paleogeography, Paleoeclimatology, Paleoecology, V. 62, p. 361-386.

Medioli, F.S., Scott, D.B., 1989b, Lacustrine thecamoebians (mainly Arcellaceans) as potential tools for palaeolimnological interpretations: In: Gray J., (ed.): "Aspects of freshwater Paleoecology and Biogeography", $i-X+1-678$, Published by: Elsevier, New York, N.Y. - [This is the same article listed as: Medioli and Scott 1989a.].

Medioli, F.S., Scott, D.B., 1989c, Methods for the collection and preparation of lacustrine Arcellaceans for micropalaeontological study: International advanced course on Paleoecology, Biostratigraphy, Paleoceanography and Taxonomy of agglutinated Foraminifera; NATO Advanced Study Institute (ASI) Programme, International Workshop on Agglutinated Foraminifera (IWAF III), Excursion guidebook (Eds.Kuhnt, W., Winkler, W., Morlotti, E. and Kaminski, M.A.), p. 154-156.

Medioli, F.S., Scott, D.B., Abbott, B.H., 1985, A method for objectively circumscribing living uniparental species of rhizopods: Abstract, Geological Society of America, v. 17, pp. 661.

Medioli, F.S., Scott, D.B., Abbott, B.H., 1987, A case study of protozoan intraclonal variability: taxonomic implications: Journal of Foraminiferal Research, v. 12, no 1, p. 28-47, 4 pls.

Medioli, F.S., Scott, D.B., Collins, E.S., Wall, J.H., 1990, Thecamoebians from the Early Cretaceous deposits of Ruby Creek, Alberta (Canada): In: Proceedings of the NATO Advanced Study Institute on Paleoecology, biostratigraphy, paleoceanography and taxonomy of agglutinated foraminifera; Hemleben, C., Kaminski, M.A., Kuhnt, W., Scott, D.B., (eds.), NATO ASI Series. Series C:

Mathematical and Physical Sciences. V. 327, pp. 793-812, D. Reidel Publishing Company. Dordrecht-Boston, International.

Medioli, F.S., Scott, D.B., Collins. E.S., McCarthy, F.M.G., 1990, Fossil thecamoebians; present status and prospects for the future: In: Proceedings of the NATO Advanced Study Institute on Paleoecology, biostratigraphy, paleoceanography and taxonomy of agglutinated foraminifera; Hemleben, C., Kaminski, M.A., Kuhnt, W., Scott, D.B., (eds.), NATO ASI Series. Series C: Mathematical and Physical Sciences. V. 327, pp. 813-839, D. Reidel Publishing Company. Dordrecht-Boston, International. 
Medioli, F.S., Scott, D.B., Wall, J.H., 1986, Early Cretaceous thecamoebians from Ruby Creek, Alberta: Abstract, Geological Society of America, Boulder, CO, United States, v.18, pp. 692

Meisterfeld, R., 1973, Die Testaceengesellschaften des Zeller Lochs (Rhizopoda, Testacea): Beiträge zur Naturkunde in Osthessen (Fulda), heft 5/6, p. 81-103.

Meisterfeld, R., 1977, Die horizontale und vertikale Verteilung der Testaceen (Rhizopoda, Testacea) in Sphagnum: Archiv für Hydrobiologie, v. 79, no 3, p. 319356.

Meisterfeld, R., 1978, Die Struktur von Testaceenzönosen (Rhizopoda, Testacea) in Sphagnum unter besonderer Berücksichtigung ihrer Diversität: Verhandlungen der Gesellschaft für Ökologie, v. 7, p. 441-450.

Meisterfeld, R., 1979a, Zur Systematik der Testaceen (Rhizopoda, Testacea) in Sphagnum. Eine REM-Untersuchung: Archiv für Protistenkunde, v. 121, p. 246-269, pls. 1-4.

Meisterfeld, R., 1979b, Clusteranalytische Differenzierung derTestaceenzönosen (Rhizopoda, Testacea) in Sphagnum: Archiv für Protistenkunde, v. 121, p. 270-307.

Meisterfeld, R., 1980, Die Struktur von Testaceenzönosen (Rhizopoda,Testacea) in Böden des Sollings: Verhandlungen der Gesellschaft für Ökologie, v. 8, p. 435-447.

Meisterfeld, R., 1981, The relation between generation time, temperature and cell size in testate amoebae (Rhizopodea): in: Dryl, S., Kazubski, S. L. and Ploszaj, J. (eds.):

"Progress in Protozoology. Abstracts of papers submitted to the VI International Congress of Protozoology, Warszawa, Poland, June 5-11, 1981": p. 243.

Meisterfeld, R., Porstendorfer, J., 1973, Rasterelektronenoptische Untersuchungen zur Schalenstruktur der Euglyphidae (Rhizopoda, Testacea): in: DePuytorac, P. and Grain, J. (eds.): "Progress in Protozoology. Abstracts of papers read at the Fourth International Congress on Protozoology, Clermont-Ferrand, 2-10 Septembre 1973": p. 277; Université de Clermont (Clermont-Ferrand).

Mercier, M., Leblanc, M., Thomas, R., Cambar, R., 1964, Observations, en microscopie électronique, sur la constitution de la thèque de quelques Euglyphidae (Rhizopodes testacés): Comptes Rendus des Séances de l'Academie des Sciences, Paris, v. 258, no 24, p. 5967-5968.

Mereschkowsky, C. von, 1878b, Studien über Protozoen des nördlichen Russland: Archiv für Mikroskopische Anatomie, v. 16, heft 2, p. 153-248, pls. 10-11.

Mereshkovskii, K. S., 1877, Étyud nad prostêishimi zhivotnmi Sêvera Rossi (Studies on the Protozoans of Northern Russia): Trud Sankt-Peterburgskago Obshchestva Estestvoisptateley- Travaux de la Sociétés Naturalistes de Saint-Pétersbourg, v. 8, p. 203-385, pls. 1-3. 
Mereshkovskii, K. S., 1878a, 0 rezul'tatakh izslêdovanii nad prostêishimi Sêvera Rossii (On the results of studies on Protozoa of Northern Russia): Trud Sankt

Peterburgskago Obshchestva Estestvoisptateley, Protokol Zasêdanii], tome 9, p. 16-19.

Meyen F. J. F., 1830, Nachträgliche Bemerkungen zur Naturgeschichte der Polypen des süssen Wassers: Isis, v. 23, columns 185-188.

Mignot, J.P., Raikov, I.B., 1990, New ultrastructural data on the morphogenesis of the test in the testacean Arcella vulgaris: European Journal of Protistology, v. 26, pp. 132-141

Mignot, J.P., Raikov, I.B., 1992, Evidence for meiosis in the testate amoeba Arcella: Journal of Protozoology, v. 39, pp. 287-289

Miller, A. A. L., Mudie, P. J., Scott, D. B., 1982, Holocene History of Bedford Basin, Nova Scotia: Foraminifera, Dinoflagellate and Pollen Records. Canadian Journal of Earth Sciences, v. 19, no. 12. In Press.

Miller, A. D., 1937, A method for culturing Arcella: in: Galstoff, P. S., Lutz, F. E., Welch, P. S. and Needham, J. G. (eds.): "Culture methods for invertebrate animals": p. 9192; Comstock Publishing Company Inc. (Ithaca, N.Y.).

Milne-Edwards, A., 1881, Compte rendu sommaire d'une exploration zoologique faite dans l'Atlantique, à bord du navire le Travailleur: Comptes Rendus des Séances de l'Academie des Sciences, Paris, v. 93, no 23,p. 931-936.

Milne-Edwards, H., 1839, Rapport sur un mémoire de M. Gervais, intitulé: Observations pour servir á l'histoire naturelle des Polypes d'eau douce, fait á l'Académie des Sciences: Annales des Science Naturelles, Zoologie, sér. 2, v. II, p. 171-185.

Minchin, E. A., 1912, An introduction to the study of the Protozoa, with special reference to the parasitic forms: p.I-XI, 1-517, 8 pls.; Edward Arnold (London). [Reprinted by same publisher in 1917 and 1922].

Minkievicz (or Minkevitsch), R., 1898, Nêkotorya nablyudenya nad prosteishikh na Bologovskoi biologicheskoi stantsi (Some observations on protozoans at the Bologoe Biological Station: Trud Sankt-Peterburgskago Obshchestva EstestvoisptateleyTravaux de la Sociétés Naturalistes de Saint-Pétersbourg, v. 29, no 7 (pagination unavailable in Zoological Record and in Royal Society Catalogue of Scientific Papers).

Minkievicz (or Minkevitsch), R., 1900, Petites études morphologiques sur le "limnoplankton": Zoologischer Anzeiger, v. 23, p. 618-623.

Mishra, A. B., Guru, B. C., Dash, M. C., 1977, Observations on the distribution of Testacea (Protozoa) in some aquatic habitats of Berhampur, Orissa, India:

Comparative Physiology and Ecology (Jadhpur, India), v. 2, no 2, p. 42-44. 
Mishra, S.R., Saksena, D.N, 1990, Seasonal abundance of the zooplankton of waste water from the industrial complex at Birla Nagar (Gwalior), India: Acta-Hydrochim.Hydrobiol. [Acta Hydrochimica-Hydrobiologica???], v. 18, pp. 215-220.

Möbius, K. A., 1889, Bruchstücke einer Rhizopoden-Fauna der Kieler Bucht: Abhandlungen der Königlichen Akademie der Wissenschaften zu Berlin, Physicalische-Mathematische Abhandlungen, Teil 2, p. 1-31, pls. 5-9.

Möbius, K. A., 1890, Verzeichnis der Rhizopoden der Kieler Bucht: Archiv für Naturgeschichte, Berlin, v. 56, no 1, p. 113-116.

Modenutti, B.E., Vucetich, M.C., 1987, Variacion espacial de los tecamebianos del Zooplankton del arroyo Rodriguez (Prov. de Buenos Aires): Limnobios, v. 2, pp. 671675

Monard, A., 1924, La Faune de la Motte. Lac de Neufchatel: Schweizerische Zeitschrift für Hydrologie, v. 2, p. 150-156.

Moniez, R. L., 1888, Faune des eaux souterraines du département du Nord et en particulier de la ville de Lille: Revue Biologique du Nord de la France, v. 1, no 3, p. 81-94.

Monteiro, M.T., Oliveira, R., Vale, C., 1995, Metal stress on the plankton communities of Sado River (Portugal): Water-Res., v. 29, pp. 695-701

Monti, R., 1899, I Protisti delle risaje. Nota la: Rendiconti del Reale Instituto Lombardo di Scienze e Lettere, ser. 2, v. 32, p. 159-164.

Monti, R., 1906, Recherches sur quelques lacs du massif du Ruitor: Annales de Biologie Lacustre, v. I, p. 120-167.

Moore, J. E. S., 1893, Observations upon Amoeba, with special reference to the existance of an apparent micro-nucleus in that organism: Annals and Magazine of Natural History, ser. 6, v. II, p. 149-154, pl. 12.

Moraczewski, J., 1961, Testacea du littoral peu profonde du lac Kisajno (Région des lacs de Mazurie): Polskie Archiwum Hydrobiologii, v. 9, p. 175-194.

Moraczewski, J., 1962, Différenciation écologique de la faune des Testace du littoral peu profonde du lac Mamry: Polskie Archiwum Hydrobiologii, v. 10, no 23, p. 333353.

Moraczewski, J., 1964, Testacea du seston des rivières Wkra et Narew: Acta Protozoologica, v. 2, p. 103-112.

Moraczewski, J., 1965, Taxocénoses des Testacea de quelques petits bassins de terrains inondables de la Narew: Acta Protozoologica, v. 3, p. 189-213.

Moraczewski, J., 1971, Quelques observations sur 1'ultrastructure du Thécamoebien: 
Arcella rotundata Play.: Protistologica, v. 6, p. 353-359.

Moraczewski, J., 1971a, La composition chimique de la coque d'Arcella discoides Ehrbg: Acta Protozoologica, v. 8, p.407-421.

Moraczewski, J., 1971b, Structure et formation de la coque d'Arcella discoides Ehrbg: Acta Protozoologica, v. 8, p. 423-437.

Moraczewski, J., Bonnet, L., 1969, Le peuplement thécamoebien de quelques tourbières dans la région de Basse-en-Chandesse (Puy-de-Dôme): Annales de la Station Biologique de Besse-en-Chandesse, no. 4, p. 291-334.

Murray, J. W., 1967, An ecological study of the Thecamoebina of Christchurch Harbour, England: Journal of Natural History, v. I, p. 377-387.

Murray, J., 1905a, The Rhizopods and Heliozoa of Loch Ness: Proceedings of the Royal Society of Edinburgh, v. 25, p. 609-615.

Murray, J., 1905b, Microscopic life of St. Kilda: Annals of Scottish Natural History, v. (?), p. 94-96.

Murray, J., 1907, Some Rhizopods and Heliozoa of the Forth area: Annals of Scottish Natural History, v. (?), p.93-96.

Murray, J., 1908, Biology of the Scottish Lochs: in: Murray, J. and Pullar, L. (eds.), "Bathymetrical Survey of the Scottish freshwater lochs": v. I, p. 275-334, (Edinburgh).

Nair, K. N., 1968, On a new species of Testacean Rhizopod (Protozoa: Euglyphidae from India: Journal of the Zoological Society of India, v. 20, p. 124-127.

Nair, K. N., Das, A. K., Mukherjee, R. N., 1971, On some freshwater Rhizopoda and Heliozoa (Protozoa) from Calcutta and its environs. Part I.: Zoological Survey of India. - no further information.

Nair, K. N., Mukherjee, R. N., 1969, On some testacean rhizopods (Protozoa: Sarcodina) of the ground and tree mosses from Calcutta and its environs: Proceedings of the National Academy of Science of India, ser. B, v. 38, p. 185-193.

Netzel, H., 1970, Mikro-Filmaufnahmen von Arcella dentata (Rhizopoda Testacea): Verhandlungsbericht der Deutschen Zoologischen Gesellschaft (supplement to Zoologischer Anzeiger), v. 64, p. 365-366.

Netzel, H., 1971a, Die Gehäusebildung bei der Thekamöbe Arcella vulgaris var. micronucleata im Forschungsfilm und im elektronenmikroskopischen Bild: Research Film, v. 7, no 4, p. 291-297.

Netzel, H., 1971b, Die Schalenbildung bei der Thekamöben-Gattung Arcella (Rhizopoda Testacea): Cytobiologie, v.3, no 1, p. 89-92. 
Netzel, H., 1971c, Form und Formbewegung beschalter Amöben (Testacea): Institut für den Wissenschaftlichen Film: Wissenschaftlicher Film C 1060/1971; Institut für den Wissenschaftlichen Film, Göttingen, p.1-14. - [Also published as Netzel, 1972d].

Netzel, H., 1971d, Morphogenese und Fortpflanzung beschalter Amöben (Testacea): Institut für den Wissenschasaftlichen Film: Wissenschaftlicher Film C 1059/1971; Institut für den Wissenschaftlichen Film, Göttingen, p.1-13. - [Also published as Netzel, 1972e].

Netzel, H., 1971e, Difflugia oviformis (Testacea). Bewegung und Fortpflanzung: Institut für den Wissenschaftlichen Film, Göttingen: pamphlet series "Encyclopaedia Cinematographica" (Wolf., G. ed.); Film E1641/1971, p. 1-10. [Also published as Netzel, 1972g].

Netzel, H., 1971f, Euglypha rotunda (Testacea). Bewegung und Fortpflazung: Institut für den Wissenschaftlichen Film, Göttingen: pamphlet series "Encyclopaedia Cinematographica" (Wolf., G. ed.); Film E1642/1971, p. I-II. - [Also published as Netzel, 1972h].

Netzel, H., 1971g, Arcella vulgaris var. multinucleata (Testacea). Bewegung und Fortpflanzung: Institut für den Wissenschaftlichen Film, Göttingen: pamphlet series "Encyclopaedia Cinematographica" (Wolf., G. ed.); Film E1643/1971, p. 1-17. - [Also published as Netzel, 1972i].

Netzel, H., 1971h, Arcella dentata (Testacea). Bewegung und Fortpflanzung: Institut für den Wissenschaftlichen Film, Göttingen: pamphlet series "Encyclopaedia Cinematographica" (Wolf., G. ed.); Film E1644/1971, p. 1-18. - [Also published as Netzel, 1972j].

Netzel, H., 1971i, Centropyxis aculeata (Testacea). Bewegung und Fortpflanzung: Institut für den Wissenschaftlichen Film, Göttingen: pamphlet series "Encyclopaedia Cinematographica" (Wolf., G. ed.); Film E1645/1971, p. 1-12. - [Also published as Netzel, 1972k].

Netzel, H., 1971j, Die Bildung des Gehauses bei Difflugia oviformis (Rhizopoda, Testacea): Archiv für Protistenkunde, v. 119, no 1, p. 1-30.

Netzel, H., 1972a, Die Bildung des Gehäusewand bei der Thekamöbe Centropyxis discoides (Rhizopoda, Testacea): Zeitschrift für Zellforschende und Mikroskopische Anatomie, v. 135, p. 45-54.

Netzel, H., 1972b, Die Schalenbildung bei Difflugia oviformis (Rhizopoda, Testacea): Zeitschrift für Zellforschende und mikroskopische Anatomie, v. 135, p. 55-61.

Netzel, H., 1972c, Morphogenese des Gehäuses von Euglypha rotunda (Rhizopoda, Testacea): Zeitschrift für Zellforschende und mikroskopische Anatomie, v. 135, p. 63-69. 
Netzel, H., 1972d, Form und Bewegung beschalter Amöben (Testacea): Publikationen zu wissenschaftlichen Filmen, Sektion Biologie (Göttingen), film C1060/1971, v. 5, no 1 , p. 40-51.

Netzel, H., 1972e, Morphogenese und Fortpflanzung beschalter Amöben (Testacea): Publikationen zu wissenschaftlichen Filmen, Sektion Biologie (Göttingen), film C1059/1971, v. 5., no 1, p. 52-62.

Netzel, H., 1972f, The origin of the shell in some Testaceous Rhizopods: Journal of Protozoology, v. 19 (Suppliment), p. 41.

Netzel, H., 1972g, Difflugia oviformis (Testacea). Bewegung und Fortpflanzung. Publikationen zu wissenschaftlichen Filmen, Sektion Biologie (Göttingen), film C1641/1971, v. 5, no 2, p. 143-150.

Netzel, H., 1972h, Euglypha rotunda (Testacea). Bewegung und Fortpflanzung. Publikationen zu wissenschaftlichen Filmen, Sektion Biologie (Göttingen), film C1642/1971, v. 5, no 2, p. 151-159.

Netzel, H., 1972i, Arcella vulgaris var. multinucleata (Testacea). Bewegung und Fortpflanzung. Publikationen zu wissenschaftlichen Filmen, Sektion Biologie (Göttingen), film C1643/1971, v. 5, no 2, p. 160-171.

Netzel, H., 1972j, Arcella dentata (Testacea). Bewegung und Fortpflanzung. Publikationen zu wissenschaftlichen Filmen, Sektion Biologie (Göttingen), film C1644/1971, v. 5, no 2, p. 171-179.

Netzel, H., 1972k, Centropyxis aculeata (Testacea). Bewegung und Fortpflanzung. Publikationen zu wissenschaftlichen Filmen, Sektion Biologie (Göttingen), film C1645/1971, v. 5, no 2, p. 180-189.

Netzel, H., 1973a, Fortpflanzung beschalter Amöeben (Rhizopoda, Testacea), film: in: DePuytorac, P. and Grain, J. (eds.), "Progress in Protozoology. Abstracts of papers read at the Fourth International Congress on Protozoology, Clermont-Ferrand, 210 Septembre, 1973)", p. 292; Université de Clermont (Clermont-Ferrand).

Netzel, H., 1973b, Morphogenese von Thekamöben (Rhizopoda, Testacea): in: DePuytorac, P. and Grain, J. (eds.), "Progress in Protozoology. Abstracts of papers read at the Fourth International Congress on Protozoology, Clermont-Ferrand, 210 Septembre, 1973)", p. 293; Université de Clermont (Clermont-Ferrand).

Netzel, H., 1973c, Musterbildung bei Thekamöben (Rhizopoda, Testacea): in: DePuytorac, P. and Grain, J. (eds.), "Progress in Protozoology. Abstracts of papers read at the Fourth International Congress on Protozoology, Clermont-Ferrand, 210 Septembre, 1973)", p. 294; Université de Clermont (Clermont-Ferrand).

Netzel, H., 1973d, Granular cement in the shell of Difflugia oviformis (Rhizopoda, Testacea): Journal of Protozoology, v. 20, no 4, p. 519-520. 
Netzel, H., 1975a, Struktur und Ultrastruktur von Arcella vulgaris var. multinucleata (Rhizopoda, Testacea): Archiv für Protistenkunde, v. 117, no 3, p. 219-245, pls. 1929.

Netzel, H., 1975b, Die Entstehung der hexagonalen Schalenstruktur bei der Thekamöbe Arcella vulgaris var. multinucleata (Rhizopoda, Testacea): Archiv für Protistenkunde, v. 117, no. 4, p. 321-357, pls. 43-51.

Netzel, H., 1975c, Morphologie und Ultrastruktur von Centropyxis discoides (Rhizopoda, Testacea): Archiv für Protistenkunde, v. 117, no 4, p. 369-392, pls. 5869.

Netzel, H., 1976a, Die Abscheidung der Gehäusewand bei Centropyxis discoides (Rhizopoda, Testacea): Archiv für Protistenkunde, v. 118, no 1, 2, p. 53-91, pls. 5-21.

Netzel, H., 1976b, Die Ultrastruktur der Schale von Difflugia oviformis (Rhizopoda, Testacea): Archiv für Protistenkunde, v. 118, no 4, p. 321-339, pls. 63-70.

Netzel, H., 1977a, Die Bildung des Gehäuses bei Difflugia oviformis (Rhizopoda, Testacea): Archiv für Protistenkunde, v. 119, no 1, 2, p. 1-30, pls. 1-14.

Netzel, H., 1977b, Die Struktur des Plättchen-Gehäuses von Euglypha rotunda (Rhizopoda, Testacea): Archiv für Protistenkunde, v. 119, no 3, p. 191-216, p1s. 1522.

Netzel, H., 1977c, Die Herstellung des Plättchen-Gehäuses bei Euglypha rotunda (Rhizopoda, Testacea): Archiv für Protistenkunde, v. 119, no 4, p. 301-324, p1s. 1-9.

Netzel, H., 1977d, Biphasic secretion during thecagenesis in the shelled rhizopod Difflugia oviformis: in: Hutner, S. H. (ed.): "The Fifth International Congress of Protozoology, New York City, 26 June- 2 July, 1977. Abstracts of the papers read at the Fifth International Congress on Protozoology, p.377.

Netzel, H., 1977e, Thekamöben: einzellige Drsen: Verhandlungen der Deutschen Zoologischen Gesellschaft, supplement to Zoologischer Anzeiger, p.266.

Netzel, H., 1979, Morphogenesis in testaceous amoeba: Europ. J. Cell Biology, v. 20, no 1, p. 117.

Netzel, H., 1980, Amöben als Baumeister: Biologie in Unserer Zeit, v. 10, no 6, p. 183-190.

Netzel, H., 1983, Gehäusewandbildung durch mehrphasige Sekretion bei der Thekamobe Netzelia oviformis (Rhizopoda, Testacea): Archiv für Protistenkunde, v. 127 , p. 351-381.

Netzel, H., Grunewald, B., 1977, Morphogenesis in the shelled rhizopod Arcella dentata: Protistologica, v. 13, no 2, p. 299-319. 
Netzel, H., Heunert, H. H., 1971, Die Zellteilung bei Arcella vulgaris var. multinucleata (Rhizopoda, Testacea): Archiv für Protistenkunde, v. 113, no 4, p. 285-292, pls. 3839.

Nigrelli, R. F., Hall, R. P., 1929, On certain cytoplasmic inclusions of Arcella vulgaris: Anatomical Record, v. 44, p. 239.

Nigrelli, R. F., Hall, R. P., 1930, Osmophilic and Neutral-red-stainable inclusions of Arcella: Transactions of the American Microscopical Society, v. 49, p. 18-22.

Nikolyuk, V. F., Gel'tser, Y. G., 1972, Pochvenne prosteishie SSSR [Soil Protozoa of the USSR]: Tashkent, p. I-311.

Nikolyuk, V. F., Tapil'skaya, N. V., 1976, Rol'prosteishikh v pochvennkh protsessakh [Role of the Protozoans in soil processes]: Tashkent, p. 1-88.

Nusslin, 0., 1884, Über einige Urthiere aus dem Herrenwieser See im badischen Schwarzwalde: Zetschrift fr wissenschaftliche Zoologie, v.40(4), p.697-712, 717724, pls.35-36.

Nuttycombe, J. W., 1937, Wheatgrain infusion: in: Galstoff, P. S., Lutz, F.E., Welch, P. S. and Needham, J. G., (eds.): "Culture methods for invertebrate animals", p. 135136. 


\section{ODE-PEL}

Odell, W. S., 1905, Notes on fresh-water Rhizopods: Ottawa Naturalist: v. 19, no 1, p. 16-20.

Ogden, C. G., 1979a, Siliceous structures secreted by members of the subclass Lobosia (Rhizopodea: Protozoa): Bulletin of the British Museum of Natural History (Zoology), v. 36, no 4, p. 203-207.

Ogden, C. G., 1979b, Comparative morphology of some pyriform species of Difflugia (Rhizopoda): Archiv für Protistenkunde, v. 122, no 1,2, p. 143-153.

Ogden, C. G., 1979c: An ultrastructural study of division in Euglypha (Protozoa: Rhizopoda): Protistologica, v. 15, no 4, p. 541-556.

Ogden, C. G., 1980a, Shell structure of three littoral species of testate amoebae from the Black Sea (Rhizopodea: Protozoa): Bulletin of the British Museum of Natural History (Zoology), v. 38, no 1, p. 16.

Ogden, C. G., 1980b, Aspects of shell structure in the genus Difflugia (Rhizopodea): Journal of Protozoology, v. 27, no 3, p. 57A-58A.

Ogden, C. G., 1980c, Notes on some Difflugiidae from Norfolk (Rhizopodea, Protozoa): Bulletin of the British Museum of Natural History (Zoology), v. 39, no 3, p. 125-138.

Ogden, C. G., 1980d, Shell structure in some pyriform species of Difflugia (Rhizopoda): Archiv für Protistenkunde, v. 123, heft 4, p. 455-470.

Ogden, C. G., 1981, Observations on clonal cultures of Euglyphidae (Rhizopodea, Protozoa): Bulletin of the British Museum of Natural History, (Zoology), v. 41, no 4, p. $137-151$.

Ogden, C. G., 1983a, The significance of the inner dividing wall in Pontigulasia Rhumbler and Zivkovicia gen. nov. (Protozoa: Rhizopoda): Protistologica, v. 19, no 2, p. 215-229.

Ogden, C. G., 1983b, Observations on the systematics of the genus Difflugia in Britain (Rhizopoda, Protozoa): Bulletin of the British Museum of Natural History (Zoology), v. 44 , no 1, p. 1-73.

Ogden, C. G., 1984a, Notes on testate amoebae (Protozoa: Rhizopoda) from Lake Vlasina, Yugoslavia: Bulletin of the British Museum of Natural History (Zoology), v. 47, no 5, p. 241-263.

Ogden, C. G., 1984b, Shell structure of some testate amoebae from Britain (Protozoa, Rhizopoda): Journal of Natural History, v. 18, p. 341-361.

Ogden, C. G., 1985, The flexible shell of the freshwater amoeba Microchlamys patella 
(Claparde and Lachmann, 1859) (Rhizopoda: Arcellinida): Protistologica, v. 21, no 1, p. 141-152.

Ogden, C. G., Couteaux, M-M., 1989, Interstitial Marine Rhizopods (Protozoa) from Littoral Sands on the East Coast of England: European Journal of Protistology, v.24, p.281-290.

Ogden, C. G., Fairman, S., 1979, Further observations on pyriform species of Difflugia (Rhizopodea): Archiv für Protistenkunde, v. 122, no 3,4, p. 372-381.

Ogden, C. G., Hedley, R. H., 1980, An atlas of freshwater testate amoebae: p. 1-222, 95 plates; British Museum (Natural History) and Oxford University Press (London and Oxford).

Ogden, C. G., Zivkovic, A., 1983, Morphological studies on some Difflugidae from Yugoslavia (Rhizopoda, Protozoa): Bulletin of the British Museum of Natural History (Zoology), v. 44, no 6, p. 341-375. ??or just Ogden

Ogden, C.G., 1987, The fine structure of the shell of Pyxidicula operculata , an aquatic testate amoeba (Rhizopoda): Archiv für Protistenkunde, v. 133, pp. 157-164

Ogden, C.G., 1988a, Morphology of the organic shell matrix of Difflugia (Rhizopoda) in culture, including modification by the addition of agglutinate particles: Archiv für Protistenkunde, v. 136, pp. 365-376

Ogden, C.G., 1988b, The role of the organic matrix in the construction of the agglutinate shell of Centropyxis discoides (Rhizopoda: Protozoa): Journal of Natural History, v. 22, pp. 137-148

Ogden, C.G., 1991a, Gas vacuoles and flotation in the testate amoeba Arcella discoides: Journal of Protozoology, v. 38, pp. 269-270

Ogden, C.G., 1991b, The biology and ultrastructure of an agglutinate testate amoeba Difflugia geosphaira sp. nov. (Protozoa, Rhizopoda): European Journal of Protistology, v. 140, pp. 141-150

Ogden, C.G., 1992, The ultrastructure of the freshwater testate amoeba Difflugia rubescens Penard (Protozoa, Rhizopoda): Archiv für Protistenkunde, v. 141, pp. 159179

Ogden, C.G., Meisterfeld, R., 1989, The taxonomy and systematics of some species of Cucurbitella, Difflugia and Netzelia (Protozoa: Rhizopoda), with an evaluation of diagnostic characters: European Journal of Protistology, v. 25, pp. 109-128.

Ogden, C.G., Meisterfeld, R., 1991, The biology and ultrastructure of the testate amoeba, Difflugia lucida Penard (Protozoa, Rhizopoda): European Journal of Protistology, v. 26, pp. 256-269. 
Opravilova, V., 1974, Testacea (Protozoa: Rhizopoda) of the river Bobrava in Moravia: Vestnik Ceskoslovenske Spolecnosti Zoologicke, v. 38, p. 127-147.

Opravilova, V., 1977a, A contribution to the ecology of Testacea of the Bítska Brook (Moravia, Czechoslovakia): Journal of Protozoology, v. 24, no 4, p. 52A-53A.

Opravilova, V., 1977b, Microzoobenthos: in: Zelinka, M., et al.: Production conditions of the polluted trout brook: Folia Facultatis Scientiarum Naturalium Universitatis Purkynianae Brunensis (Brno), Biologia, v. 18, p. (?).

Opravilova, V., 1980, Príspevek k poznání ekologie krytenek (Rhizopoda: Testacea) potoka Bítska (Morava, CSSR) (Contribution to the knowledge of the ecology of the Testacea (Rhizopoda) of the Bítska Brook (Moravia, Czechoslovakia): Prírodovedn Sborník Zípadomoravského Muzea v Trebíci, v. II, p. 5-16.

Opravilova, V., 1983, A contribution to the knowledge of Testacea (Protozoa: Rhizopoda) of the Jihlava River near Hurbsice: Scripta fac Sci. Nat. Univ. Purk. Brun. Biologia, v. 13, no 1, p. 23-32.

Opravilova, V., 1986, Testacea (Protozoa: Rhizopoda) in the epilithion of the lotic stretch of running waters of different degrees of saprobity (Czechoslovakia): ACTAHYDROCHIM.-HYDROBIOL., v. 14, pp. 667-672

Opravilova, V., 1989, Some information on testate amoebae from Spitsbergen: FAUNA-NORV.,-SER., v. 10, pp. 33-37

Opravilova,V., Stepanek,M., 1980, Some less frequent forms of Testacea (Protozoa: Rhizopoda) in small streams of the CSSR: Vestn.-Cesk.-Spol.-Zool., v. 44, pp. 128138

Orlov, Y. A., 1959, Otryad Testacea (Thecamoebina) (Order Testacea (Thecamoebina)): in: Orlov, Y. A. (gen. ed.), "Osnov paleontologii, Tome 1: Orlov, Y. A., Rauzer-Chernousova, D. M. and Fursenko, A. V. (eds.), Obshchaya chast'.

Prosteishie" p. 114; Izdatel'stvo Akademii Nauk SSSR (Moskva), [Fundamentals of Paleontology. A manual for paleontologists and geologists of the USSR: Orlov, Y. A., Rauzer-Chernousova, D. M. and Fursenko, A. V. (eds.), General part. Protozoa]. translated as: Orlov, 1962.

Orlov, Y. A., 1962, Order Testacea (Thecamoebina): in: Orlov, Y. A. (gen. ed.), "Fundamentals of Paleontology. A manual for paleontologists and geologists of the USSR: Orlov, Y. A., Rauzer-Chernousova, D. M. and Fursenko, A. V. (eds.), General part. Protozoa", p. 111; Israel Program for Scientific Translations (Jerusalem). translated from: Orlov, 1959.

Orr, H. D., 1954, Quantitative studies of Protozoan populations from two areas of Pymatuning Lake, Pennsylvania: Ecology, v. 35, no 3, p.(?).

Owen, G., III, Jones, E. E., 1976, Nebela tuberculata comb. nov. (Arcellinida), its history 
and ultrastructure: Journal of Protozoology, v. 23, no 4, p. 485-487.

Oye, P. 1956b, On the Thecamoeben fauna of New Zealand with descriptions of four new species and biogeographical discussion: Hydrobiologica, v. 8, no 1,2, p. 16-37.

Oye, P. van, 1922a, Contribution à la conaissance de la flore de la faune microscopique des Indes Néerlandaises: Ann. Biol. Lacustre, v. 11, no 1, p. 130-150.

Oye, P. van, 1922b, Zur biologie des potamoplanktons aus Java: Internationale Revue der Gesamten Hydrobiologie und Hydrographie, v. 10, p. 363-393.

Oye, P. van, 1923, Deux Rhizopodes nouveaux du Congo belge: Revue Zool. Bot.Afr., v. 12, p. (?).

Oye, P. van, 1924, Zur biologie des potamoplankons aus Java: Internationale Revue der Gesamten Hydrobiologie und Hydrographie, v. 12, p. 48-49.

Oye, P. van, 1926a, Six Rhizopodes nouveau du Congo belge: Arch. Zool. exp. Gén., Notes et Revues, v. (?), no 3, p. 64-74.

Oye, P. van, 1926b, Potamoplankton de la Ruki au Congo Belge et des pays chauds en général: Internationale Revue der Gesamten Hydrobiologie und Hydrographie, v. 16, p.1-50.

Oye, P. van, 1927, Rhizopoden en Heliozoën van Belgish Kongo:

Natuurwetenschappelijke Tijdschrift, v. 9, p. 4-18. ??thecas Oye, P. van, 1932a, Neue Rhizopoden aus Afrika: Zoologischer Anzeiger, v. 29, p. 323-328.

Oye, P. van, 1932b, Rhizopoda from South Africa: Revue de Zoologie et de Botanique Africaines, v. 31, p.54-73.

Oye, P. van, 1933, Rhizopodes du district sub-alpin de la Belgique: Archiv für Naturgeschichte, Abt. B, N.F. 2, p.538-573.

Oye, P. van, 1936, Rhizopoden uit het mos van Meijendel: Levende Natuur, v. 40, p. 215-221, 240-246.

Oye, P. van, 1938a, Rhizopoden von Haiti. An investigation of some Hispaniolan Lakes (Dr. R. M. Bonds Expedition): Archiv für Hydrobiologie, v. 32, p. 320-332.

Oye, P. van, 1939a, Weteinschapelijke resultaten det studiereis van Prof. Oye of ljslend: Biologisch Jaarboek Dodonaea (Gent), v. (?) p. (?).

Oye, P. van, 1939b, Die Rhizopoden von Thingvallavatn und Umgeburg: Biologisch Jaarboek Dodonaea (Gent), v. ?, p. 171-185.

Oye, P. van, 1941, Die Rhizopoden des Sphagnetums bei Krisuvik auf Island: Biologisch Jaarboek Dodonaea (Gent), v. (?), p. 284-305. 
Oye, P. van, 1944, Au sujet de la distribution géographique des Rhizopodes:

Biologisch Jaarboek, v. 11, no 2, p. 83-91.

Oye, P. van, 1948a, De Rhizopodia van België: Biologisch Jaarboek Dodonaea (Gent), v. 15, p. 167-172.

Oye, P. van, 1948b, Rhizopodes du parc Albert: Institut Parcs Nationaux Congo Belge (1948), p. 1-45.

Oye, P. van, 1949, Rhizopodes de Java: Bijdragen tot de Dierkunde, v. 28, p. 327352, p1. 28.

Oye, P. van, 1950, Les Protistes comme indicateurs biogéographies: Bull. Soc. Bot. Nord de la France, v. 2, no 3, p. ?.

Oye, P. van, 1951, Au sujet des Rhizopodes du Grand-Duché de Luxembourg: Biologisch Jaarboek Antwerpen, v. 18, p. 32-47. ??pages Oye, P. van, 1951b, Is Arcella amorpha identical with Arcella apicata: Hydrobiologica, v. (?), p. (?).

Oye, P. van, 1952, Rhizopoda Vénézuélas: Deutscher Verlag der Wissenschaften Berlin, p. 329-360.

Oye, P. van, 1953, Faune Rhizopodique de 1'Étang de Beernem: Biologisch Jaarboek Dodonaea (Gent), v. 20, p. 154-205.

Oye, P. van, 1956a, Rhizopoda Venezuelas, mit besonderer Berücksichtigung ihrer Biogeographie: in: Ergebnisse der Deutschen Limnologischen VenezuelaExpedition 1952, v. I, p. 329-360.

Oye, P. van, 1956c, Rhizopodes de Belgique, Biologisch Jaarboek Dodonaea (Gent), v. 23, p. (?).

Oye, P. van, 1958, Étude sur les Rhizopodes des marais du Sud-ouest d'Uvira (Congobelge): Hydrobiologia, v. 10, p. 85-127, pls. 1-5.

Oye, P. van, 1959, Faune Rhizopodique du plateau de Kundulungu (Congo Belge): Hydrobiologia, v. 13, no 3, p. 239-286.

Oye, P., 1938, Twee veev-profielen uit Zuid-Luxembourg: Biologisch Jaarboek Dodonaea (Gent), v. (?), p. 425-434.

Padovani, C., 1911, II plancton del Fiume Po, contributo allo studio del plancton fluviale: Zoologischer Anzeiger, v. 37, p. 99-104.

Page, F.C. (1966) Cryptodifflugia operculata n. sp. (Rhizopodea:Arcellinida, Crypodifflugiidae) and the status of the genus Cryptodifflugia. Transactions of the American Microscope Society, 85 506-515.

Page, F. C., 1976, An illustrated key to freshwater and soil amoebae with notes on 
cultivation and ecology: Freshwater Biological Association Scientific Publication v. 34, p. 1-155, (Ambleside).

Page, F. C., 1979, Two genera of marine amoebae (Gymnamoebia) with distinctive surface structures: Vannella Bovee 1965 and Pseudoparamoeba n. gen., with two new species of Vannella: Protistologica, v. 15, no. 2, p. 245-257.

Parenti, G., 1992, Le Tecamebe nei sedimenti nel Lago Superiore di Mantova: (Unpublished M. Sc. thesis) Università degli studi di Parma, 95 pp.

Parfitt, E., 1869, On the Protozoa of Devonshire: Transactionas of the Devonshire Association, v. 3, p. 60-67.

Parker, F., 1952a, Foraminifera species off Portsmouth, New Hampshire: Bulletin of the Harvard Museum of Comparative Zoology, v. 106, no. 9, p. 391-423, pl. 1-6.

Parker, F., 1952b, Foraminiferal distribution in the Long Island Sound- Buzzards Bay area: Bulletin of the Harvard Museum of Comparative Zoology, v. 106, no 10, p. 427-473, pl. 1-5.

Parker, F., Phleger, F. B, Peirson, J. F., 1953, Ecology of Foraminifera from San Antonio Bay and environs, Southwest Texas: Cushman Foundation for Foraminiferal Research, Special Publication no 2, p. 1-75, pls. 1-4.

Parona, C., 1880a, Prime ricerche intorno ai Protisti del lago d'Orta, con cenno della loro corologia italiana: Bollettino Scientifico (Pavia), v. 1, no 2, p. 17-26.

Parona, C., 1880b, Intorno alla corologia dei Rizopodi: Bollettino Scientifico (Pavia), v. I, no 2, p. 43-50.

Parona, C., 1880c, Title (?), Bollettino Scientifico (Pavia), v. I, no 2, p. 211 (?).

Parona, C., 1882, Materiali per la fauna dell'Isola di Sardegna.I. Protisti della Sardegna: Bollettino Scientifico (Pavia), v. I, no 4, p. 44-58.

Parona, C., 1883, Essai d'une protistologie de la Sardaigne: Archives des Sciences naturelles, ser. 3, v. 10, p. 225-243, pl. 2.

Parona, C., 1884, I Protisti della Sardegna: Bollettino Scientifico (Pavia), v. 2, no 2, p. 53-59.

Pateff, P., 1926a, Fortpflanzungerscheinungen bei Difflugia mammillaris Penard und Clypeolina marginata Penard: Archiv für Protistenkunde, v. 55, no 3, p. 516-544, pls. 15-16.

Pateff, P., 1926b, Susswässer-Rhizopoden aus der Holhe Salzlocher (Schlesien): Mitteilungen über Hohlen und Karstforschung, v. 2, p. 46-49.

Patterson, R.T., Barker, T., and Burbidge, S.M., 1996,Arcellaceans (Thecamoebians) 
as Proxies of Arsenic and Mercury Contamination in Northeastern Ontario Lakes. Journal of Foraminiferal Research, 26:172-183.

Patterson, R. T., MacKinnon, K. D., Scott, D. B., Medioli, F. S., 1985, Arcellaceans (Thecamoebians) in small Lakes of New Brunswick and Nova Scotia: Modern distribution and Holocene stratigraphic changes: Journal of Foraminiferal Research, v. 15 , no 2 , p. $114-137$.

Patterson, R.T., 1983, Arcellaceans of several small lakes in southwestern New Brunswick, their modern distribution and stratigraphic importance. Unpublished Bachelor of Arts Honours Thesis, Dalhousie University, 59 pages.

Patterson, R.T., 1987, Arcellaceans and foraminifera from Pleistocene Lake Tecopa, California: Journal of Foraminiferal Research, v. 17, pp. 333-343.

Paulson, B., 1953, Some rhizopods associations in a Swedish mire: Oixos, v. 4, no 2, p. 151-165.

Pawlikowski, M., Ralska-Jasiewiczowa, M., Schönborn, W., Stupnika, E., Szeroczyska, K., 1982, Woryty kolo na Pojezierzu Olsztyskim - Historia olinnoci i rozwój jeziora w ciau ostatnich 12.000 lat (Woryty- near Gietrzwald, Olsztyn Lake District, N.E.Poland - vegetetional history and lake development during the last 12,000 years): Acta Palaeobotanica, v. 22, no 1, p. 85-116.

Peach, M., 1955, Soil fungi that prey on Protozoa in: McKevan, K. (ed); "Soil Zoology": Proceedings of the University of Nottingham Second Easter School in Agricultural Science, Butterworths Scientific Publications, London; p. 302-310.

Pearl, R., Dunbar, F. J., 1903, Variation and correlation in Arcella: Biometrika, v. 2, no 3, p. 321-337.

Peltier, A., 1835, Observations microscopiques sur les animalcules: Bulletin de la Société des Sciences naturelles de France, p. 92-95. - We are not certain that this paper deals with thecamoebians.

Peltier, A., 1836, Note sur un mode de propagation des Arcelles vulgaires et scutelliformes (Arcellae aculeatae (sic), Ehr.): Extraits des Procès-verbaux des Séances de la Société Phlomathique de Paris, p. 63-64. 


\section{PEN-RUZ}

Penard, E., 1889, Einige Bemerkungen über die in der Umgegend von Wiesbaden vorkommenden Protozoen: Jarbuch der nassavischen Vereins für Naturkunde, v. 42, p. 141-146.

Penard, E., 1890a, Études sur les Rhizopodes d'eau douce: Mémoires de la Société de Physique et d'Histoire Naturelle de Genève, v. 31, no 2, part 1, p. 1-230, pl. 1-11.

Penard, E., 1890b, Catalog der nackten und schalentragenden Rhizopoden von Wiesbaden: Jarbuch der nassavischen Vereins für Naturkunde, v. 43, p. 67-72.

Penard, E., 1891a, Contributions à l'étude des Rhizopodes du Léman: (Bibliothéque Universelle); Archives des Sciences physique et naturelles, ser. 3, v. 26, p. 134156, pl.2.

Penard, E., 1891b, Rocky Mountain Rhizopods: American Naturalist, v. 25, p. 10701083.

Penard, E., 1893, Pelomyxa palustris et quelques autres organismes inférieurs: (Bibliothéque Universelle); Archives des Sciences physique et naturelles, ser. 3 , v. 29 , p. $161-180$, pl. 3.

Penard, E., 1899a, Les Rhizopodes de faune profonde dans le lac Léman: Revue Suisse de Zoologie, v.7, no 1, p. 1-142, pls. 1-9.

Penard, E., 1899b, Sur la croissance supposée de la coquille chez les Thécamoebiens: (Bibliothéque Universelle); Archives des Sciences physique et naturelles, ser. 4, v. 7 , p. 249-271.

Penard, E., 1899c, Experiences sur les pseupodes detachès de Rhizopodes: (Bibliothéque Universelle); Archives des Sciences physique et naturelles, ser. 4, v. 8, p. 90.

Penard, E., 1900, Essais de méritomie sur quelques Difflugies: Revue Suisse de Zoologie, v. 8, p. 477-490.

Penard, E., 1901, Notes complémentaires sur les Rhizopodes du Léman: Revue Suisse de Zoologie, v. 9, p. 225-241.

Penard, E., 1902, Faune rhizopodique du bassin du Léman: p. 1-714 Henry Kündig (Genue).

Penard, E., 1903a, Notice sur les Rhizopodes du Spitzberg: Archiv für Protistenkunde, v. 2, no 2, p. 238-282.

Penard, E., 1903b, Sur quelques protistes voisins des Héliozoaires ou des Flagellates: Archiv für Protistenkunde, v. 2, no 2, p. 283-304. 
Penard, E., 1904, Quelques nouveaux Rhizopodes d'eau douce: Archiv für Protistenkunde, v. 3, p. 391-422.

Penard, E., 1905a, Les Sarcodinés des grands lacs: p. 1-134; Henry Kündig, éditeur (Genève).

Penard, E., 1905b, Sarcodinés: in: Musée d'Histoire Naturelle de Genève: "Catalogue des Invertébrés de la Suisse": fasc. I, p. I-XII, 1-164.

Penard, E., 1905c, Notes sur quelques Sarcodinés. 1e partie: Revue Suisse de Zoologie, v. 13, no 3, p. 585-616, pls. 13-14.

Penard, E., 1905d, Sur les Sarcodinés du Loch Ness: Proceedings of the Royal Society of Edinburgh, v. 25, part 2, no 8, p. 593-608.

Penard, E., 1906a, Notes sur quelques Sarcodine. 2e partie: Revue Suisse de Zoologie, v. 14, no 2, p. 109-141, p1. 4.

Penard, E., 1906b, Étude sur la Clypeolina marginata: Archiv für Protistenkunde, v. 8, no 1, p. 66-85.

Penard, E., 1907a, Recherches biologiques sur deux Lieberkühnia: Archiv für Protistenkunde, v. 8, no 2, p. 225-258.

Penard, E., 1907b, On the collection and preservation of fresh water rhizopods: Journal of the Quekett Microscopical Club, v. 10, p. 107-116.

Penard, E., 1907c, On some Rhizopods from the Sikkim Himalaya: Journal of the Royal Microscopical Society, p. 274-278, pl. 14.

Penard, E., 1908, Recherches sur les Sarcodinés de quelques lacs de la Suisse et de la Savoie: Revue Suisse de Zoologie, v. 16, no 3, p. 441-471, pl. 17.

Penard, E., 1909, Sur quelques Rhizopodes des mousses: Archiv für Protistenkunde, v. 17 , no 2, p. 258-296.

Penard, E., 1910, Rhizopodes nouveaux: Revue Suisse de Zoologie, v. 18, no 4, p. 929-940, pl. 8.

Penard, E., 1911a, Rhizopodes d'eau douce: in: Shackleton, E.H. (gen. ed.), "British Antarctic Expedition 1907-9, under the command of Sir E. H. Shackleton, C. V. O. Reports on the scientific investigations.": v. I, "Biology", Murray, J. (ed.), part 6, p. 203262, pls. 22,23; William Heinemann (London).

Penard, E., 1911b, On some Rhizopods from Sierra Leone: Journal of the Quekett Microscopical Club, ser. 2, v. II, p. 299-306, pls. 9-10.

Penard, E., 1912a, Scotia collections - Further note on microscopic life on Gough Island, South Atlantic Ocean-"Rhizopoda": in Scottish Oceanographic Laboratory, 
Zoology, Scottish National Antarctic Expedition. Report on the scientific results of the voyaqe of S.Y. Scotia during the years 1902, 1903, and 1904, under the leadership of W.S. Bruce: v. 6, no 10, p. 267-269. (Edinburqh).

Penard, E., 1912a, Scotia collections - Further note on microscopic life on Gough Island, South Atlantic Ocean- Rhizopoda": Proceedings of the Royal Physical Society (Edinburgh), v. 18, no 4, p. 244-247.

Penard, E., 1912c, Notes sur quelques Sarcodinés. 3me oartie: Revue Suisse de Zooloqie, v. 20, no 1, p. 1-29, pls. 1, 2.

Penard, E., 1922, Les Protozoaires considérés sous le rapport de leur perfection organique: 95 p., Georg and Cie (Genève).

Penard, E., 1938, Les infiniment petits dans leurs manifestations vitales: 212 p., Georg and Cie (Genève).

Perry, S. T., 1891, Freshwater Rhizopods of Oakland County, Michigan: Proceedings of the American Microscopical Society, v. 12, p. 94-96.

Perty, J. A. M., 1855, Herr Geheimrath Ehrenberg in Berlin und die Infusorienkunde. Ein Sendschreiben: 3 p., (Bern ?).

Perty, M., 1848a, Bemerkungen über die Bacillarien: Mittheilungen der Naturforschenden Gesellschaft in Bern, p. 160-164. thecampebians (?)

Perty, M., 1848b, Ueber die Entwickelung einiger Infusorien: Mittheilungen der Naturforschenden Gesellschaft in Bern, p. 193-208. thecampebians (?)

Perty, M., 1849a, Über verticale Verbreitung mikroskopischer Lebensformen: Mittheilungen der Naturforschenden Gesellschaft in Bern, no 146-149, p. 17-45.

Perty, M., 1849b, Mikroskopische Organismen der Alpen und der Italienischen Schweiz: Mittheilungen der Naturforschenden Gesellschaft in Bern, no 164,165, p. 153-176.

Perty, M., 1849c, Eine physiologische Eigenthümlichkeit der Rhizopodensippe Arcella Ehr.: Mittheilungen der Naturforschenden Gesellschaft in Bern, p. 124-126.

Perty, M., 1852a, Zur Kenntniss Kleinster Lebensformen nach Bau, Funktionen, Systematik, mit Spezialverzeichniss der in der Schweiz beobachteten n: p. I-VIII, 1-228, pls. 1-17; Jent und Reinert (Bern).

Perty, M., 1852b, System der Infusorien: Mittheilungen der Naturforschenden Gesellschaft in Bern, no 231, 232, p. 57-68.

Petri, S., 1979, Observations on transport of organisms by marine currents: Anais da Academia Brasileira de Ciencias (Formerly AABRA)-CISTI, v. 51, no 3, p. 517-522. 
Peus, F., 1932, Die Tierwelt der Moore, unter besonderer Berücksichtigung der Europäischen Hochmoore: in: Bülow, K., von (ed.), "Handbuch der Moorkunde": vol. III, p. I-VIII, 1-277; Borntraeger (Berlin).

Phillips, F. W., 1883, Notes on the Protozoans of Hertfordshire: section Pantostomata: Transactions of the Hertfordshire Natural History Society, v. 2, no 3, p. 115-125.

Pinel-Alloul,B., Magnin,E.- 1978, Zooplancton d'une tourbiere reticulee du territoire de la baie de James: Naturalist Canadien, v. 105, pp. 19-35

Pinto, C., 1925, Protozoarios observados no Brasil: Mem. Inst. Oswaldo cruz, v. 18, p. 211-302, pls. 16-28.

Piskol, H., 1969, Ameby skorupkowe (Testacea) mchów Kamieniolomów Strzeblowa: Zeszyty przyr., v. 9, p. 87-98.

Pitelka, D. R., 1963, Electron-microscopic structure of Protozoa: in: "International series on pure and applied biology", v. 13, p. I-X, 1-269, 24 plates; Pergamon Press (Oxford).

Playfair, G. I., 1914, Contributions to a knowledge of the biology of the Richmond River: Proceedings of the Linnean Society of New South Wales, v. 39, part 1 (no 153), p. 93-151, pls.2-8.

Playfair, G. I., 1918, Rhhizopods of Sydney and Lismore: Proceedings of the Linnean Society of New South Wales, v. 42, part 4(no 168), p. 633-675, pls. 34-41.

Poche, F., 1913, Das System der Protozoen: Archiv für Protistenkunde, v. 30, p. 125321.

Popoff, M., 1912, Ueber die geschlechtliche Fortpflanzung von Euglypha alveolata Duj.: Archiv für Protistenkunde, v. 25, no 1, p. 8-26, pls. 1,2.

Prandtl, H., 1907, Der entwicklungskreis von Allogromia sp.: Archiv für Protistenkunde, v. 9, no 1, p. 1-20, pl. 1.

Prenant, M., Bobin, G., 1956, Bryozoaires. Première Partie: Entoproctes, Phylactolèmes, Cténostomes: in: Faune de France, v. 60, p. I-III, 1-398; Paul Lechevalier (Paris).

Pritchard, A., 1834, The natural history of animalcules: containing descriptions of all the known species of Infusoria: p. 1-196, 6 plates; (Whittaker and Co.) London.

Pritchard, A., 1841 (reprinted in: 1842, 1845, 1849), A history of Infusoria, living and fossil, arranged according to "Die Infusionsthierchen" of Ehrenberg, containing coloured engravings illustrative of all the genera, and descriptions of all the species in that work, with several new ones. To which are appended "An account of those recently discovered in the chalk formations": p. I-IX, 1-439, 12 plates; 
(Whittaker and Co.) London. (considered by some as the 2nd edition of Pritchard 1834).

Pritchard, A., 1851, A general history of animalcules: p. 1-83, 12 plates; (London).

Pritchard, A., 1852, A history of infusorial animalcules, living and fossil: p. I-VIII, 1706, 24 plates (a new edition enlarged by J. T. Arlidge and A. Pritchard); Whittaker and Co. (London). often considered as 3rd. edition of Pritchard 1834, 1841.

Pritchard, A., 1861, A history of Infusoria, including the Desmidiaceae and Diatomaceae, British and foreign: p. I-XII, 1-968, pls. 1-40; Whittaker and Co. (London) fourth edition, enlarged and revised by J. T. Arlidge, W. Archer, J. Rafts, W. C. Williamson and A. Pritchard.

Prowazek, S., von, 1900a, Cystenbildung bei den Protozoen: Zeitschrift für angewandte Mikroskopie, v. 5, no 10, p. 269-274, pl. 2.

Prowazek, S., von, 1900b, Protozoenstudien II: Arbeiten des Zoologischen Instituten der Universit\{t Wein, v. 12, no 3, p. 243-300, pls. 17,18.

Prowazek, S.,von, 1909, Taschenbuch der mikroskopischen Technik der Protistenuntersuchung:.p. (?), Leipzig.

Pujos, M., 1973, Les biocoenoses de foraminiferes benthiques et de thecamoebiens dans le complexe Garonne-Dordogne-Gironde, manifestations de l'influence fracteurs de l'environnement sur les microfaunes: Bulletin de l'Institut de Geologie du Bassin d'Aquitaine, v. 13, pp. 3-19.

Purkyne, J. E., 1855a, O Rhizopodech (korenonozeich): Ziva, v. 3, p. 109-115. thechamoebians (?).

Purkyne, J. E., 1855b, Porovnáni tvaru Rhizopodu skorepnutch: Ziva, v. 3, p. 210-216. - thechamoebians (?).

Pussard, M., Senaud, J., Pons, R., 1977, Observations ultrastructurales sur Gocevia fonbrunei Pussard 1965 (Protozoa, Rhizopodea): Protistologica, v. 13, no 2, p. 265285.

Puytorac, P. de, Mignot, J. P., Grain, J., Groliere, C. A., Bonnet, L., Couillard, P., 1972, Premier relevé de certains groupes de protozoaires libres sur le territoire de la Station de Biologie de 1'Université de Montréal (Saint-Hyppolyte, Comté de Terrebonne, Qubec): Naturaliste Canadien, v. 99, p. .417-440.

Raikov, I.B., Karadzhan, B.P., Kaur,R., Mignot,J.P., 1989, Nuclear fine structure at interphase and during encystment in two forms of the testacean Arcella vulgaris:

European Journal of Protistology, v. 24, pp. 369-380

Raikov, I.B., Mignot, J.P., 1991, Fine-structural study of mitosis in the testacean Arcella vulgaris Ehrbg: European Journal of Protistology, v. 26, no. 3-4, pp. 340-349 
Raju, N. S., Suxena, M. R., 1979, Algae and Testacea of the Cho-Qyu (Himalayas) Expedition II; Cyanophyta, Chlorophyta, Euglenophyta, Chrysophyta and Testacea: Hydrobiologia, v. 67, no 2, p. 141-160.

Rampi, L., 1947, Fauna di Romagna (Collezione Zangheri), Rizopodi Testacei - Ima Contribuzione: Atti della Societá Italiana di Scienze Naturali e del Museo Civico di Storia Naturale in Milano, v. 86, no 1,2, p. 71-77.

Rampi, L., 1950, Su alcuni laghetti alpini del Massiccio dell'Abisso: Bollettino di Pesca, Piscicultura e Idrobiologia, new ser., v. 5, no 2, p. 207-224.

Rampi, L., 1957, I Rizopodi Testacei del laghetto di Terrasole (Alpi Liguri): Atti della Societá Italiana di Scienze Naturali e del Museo Civico di Storia Naturale in Milano, v. 96, p.192-199.

Reid, G. K., 1961, Ecology of inland waters and estuaries: Reinhold (New York),p. i$x v i+1-375$.

Reinhardt, E.G., Dalby, A., Kumar, A., Patterson, R.T., 1998, Utility of Arcellacean Morphotypic Variants as Pollution Indicators in Mine Tailing Contaminated Lakes Near Cobalt, Ontario, Canada: Micropaleontology ${ }_{2}$ v. 44 no 2.

Reukauf, E., 1912, Zur Encystierung von Euglypha alveolata: Zoologischer Anzeiger, v. 39, p. 372-375.

Reuss, A. E., 1862, Entwurf einer systematischen Zusammenstellung der Foraminiferen: Sitzungsberichte der Kaiserlichen Akademie der Wissenschaften zu Wien, mathematish-naturwissenschaftlichen Classe, v. 44, p. 355-396.

Reynolds, B. D., 1923, Inheritance of double characteristics in Arcella polypora Penard: Genetics, v. 8, p. 477-493.

Reynolds, B. D., 1937, Culturing Arcellae: in Galtsoff, P. S., Lutz, F. E., Welch, P. S. and Needham, J. G., (eds.), "Culture methods for invertebrate animals", p. 92; Comstock Publishing Co. Inc. (Ithaca, N.Y.).

Rhumbler, L., 1891, Beiträge zur Kenntnis der Rhizopoden. I. Über Entstehung und sekundäres Wachsthum der Gehaüse einiger Süsswasserrhizopoden: Zeitschrift für Wissenschaftliche Zoologie, v. 52, no 4, p. 515-550, pl. 32.

Rhumbler, L., 1895a, Beiträge zur Kenntnis der Rhizopoden (Beitrag III, IV und V): Zeitschrift für Wissenschaftliche Zoologie, v. 61, no 1, p. 38-110, pls. 4, 5.

Rhumbler, L., 1895b, Entwurf eines natürlichen Systems der Thalomophoren: Nachrichten der Gesellschaft für Wissenschaft Göttingen, mathematischephysikalisch Klasse, Nr. 1, p. 51-98.

Rhumbler, L., 1898a, Physikalische Analyse von Lebenserscheinungen der Zelle. I. 
Bewegung, Nahrungsaufnahme, Defäkation, Vacuolen-Pulsation, und Gehäusebau bei lobosen Rhizopoden: Archiv für Entwicklungsmechanik, v. 7, no 1-3, p. 103-350, pls. 6,7 .

Rhumbler, L., 1898b, Zelleib-, Schalen- und Kernverschmelzungen bei den Rhizopoden und deren wahrscheinliche Beziehungen zu phylogenetischen Vorstufen der Metazoenbefruchtung: Biologisches Zentralblatt, v. 18, p. 21-26, 33-38, 69-86, 113-130.

Rhumbler, L., 1902, Die Aggregatzustand und die physikalischen Besonderheiten des lebenden zellinhaltes. II. Thiel: Zeitschrift für Allgemeine Physiologie, v. 2, p. 183340.

Rhumbler, L., 1903, Systematische Zusammenstellung der recenten Reticulosa (Nuda und Foraminifera). I. Theil: Archiv für Protistenkunde, v. 3, no 1, p. 181-294.

Rhumbler, L., 1907, Title??, Journal (?),.v. 68, part 2(3), p.(?) at least 54.

Rhumbler, L., 1923, I.(?) Unterstamm: Plasmodroma. 1. Klasse: Rhizopoda oder Sarkodina, Wurzelfsser; II.(?) Erstes Unterreich der Tiere. Einziger Stamm dieses Unterreiches: Protozoa-Urtiere: in: Kkental, W., and Krumbach, Th. (eds.): Handbuch der Zoologie. Eine Naturgeschichte der Stamme des Tierreiches: v.l (I), p. 51-114.

Rhumbler, L., 1928, Amoebozoa und Reticulosa: in: Grimpe, G. and Wagler, E., (eds.): "Die Tierwelt der Nord- und Ostsee", v. II, part a, p. 1-26.

Rhumbler, L., 1935, Rhizopoden der Kieler Bucht, gesammelt durch A. Remane, Teil I: Schriften des Naturwissenschaftlichen Veriens für Schleswig-Holstein (Keil), v. 21, p. 143-194, pls. 1-9.

Richard, A., 1824, ??: in: Bory de St.-Vincent, J. B. (ed.), "Dictionnaire classique d'histoire naturelle", Tome 6, p. ??; Rey and Gravier, and Baudouin Frères (Paris).

Richters, F., 1908a, Beitrag zur Kenntnis der Moosfauna Australiens und der Inseln des Pazifischen Ozeans: Zoologische Jahrbuch, Abt. systematik, v. 26, p. 196-213, pl. 17.

Richters, F., 1908b, Moosbewohner: in: Nordenskjöld, O., (ed.): "Wissenschaftlichen Ergebnisse der Schwedischen Südpolar-Expedition 1901-1903": v. 6, "Zoologie II", p. 1-16, 1 plate; (Stockholm).

Rioja, E., 1942, Estudios hydrobiologicos VII. Apuntes para el estudio de la laguna de San Felipe Xochiltepec (Puebla) I: Annales del Instituto de Biologia, Universidad de Mexico, v. 13, no 2, p. 503-517.

Root, F. M., 1918, Inheritance in the asexual reproduction of Centropyxis aculeata: Genetics, v. 3, p. 173-206. 
Rosa, K., 1957, Bodenmikrofauna und mikrofauna im Fichtenbestande am Praded (Altvater): Prirodovedna Sbornik Ostraveskeho Kraje, v. 18, p. 7-75.

Rosa, K., Lhotsky, 0., 1971, Edaphische Algen und Protozoen im Isergebirge Tschechoslowakei: Oikos, v. 22, p. 21-29.

Rossolimo, L. L., 1927, Atlas ostatkov zhivotnykh organizmov v torfakh i sapropelkh (Atlas tierischer Überreste in Torf und Sapropel): p. 1-48, pls. 1-9; (Moskova).

Rudwick, M.J.S., 1967, Class Rhizopodea - Class Reticularea, subclass Filosia: in: Harland, W.B., et al. (eds.): The fossil record: a symposium with documentation: p.292-293 (fig.8.1), 298-299, 301 (fig.8.2b); Geological Society of London, London.

Ruitenburg, G. J., Davids, C., 1977, Thecamoeba in succession series of some peatbog vegetations: Hydrobiological Bulletin (Amsterdam), v. Il, p. 22-24.

Ruzick, E., 1982, Die subfossilen Testaceen des Krottensees (Salzburg, Osterreich): Limnologica (Berlin), v. 14, no 1, p. 49-88. 


\section{SAB-SCH}

Sabri, A.W., 1988, Seasonal variation of testaceous amoebae (Sarcodina, Protozoa) population in the river Tigris: ARAB-GULF-J.-SCI.-RES.-B:-AGRIC.-BIOL.-SCI, v. 6, pp. $439-448$

Sacchi, M., 1888a, Intorno ai Protisti dei muschi ed al loro incistamento: Bollettino Scientifico (Pavia), v. 3, anno 10, no 2, p. 35-56. translated in Sacchi 1888b.

Sacchi, M., 1888b, Sur les Protistes des mousses et leur enkystement: Journal de Micrographie, v. 12, p. 340-347, 376-380, 403-409.

Sacchi, M., 1890a, I Protozoi terricoli. Nota preventiva: Bollettino Scientifico (Pavia), v. 3, no 3, p. 65-68.

Sacchi, M., 1890b, Les Protozoaires terricoles: Journal de Micrographie, v. 14, p. 107-109.

Saedeleer, F., 1894, Über die systematische Stellung und Fortpflanzung von Yalopus (Gromia dujardinii Schultze): Sitzungsberichte der Gesellschaft der naturforschenden Freunde zu Berlin, p. 14-22. (Reprinted as: Schaudinn, 1911a).

Saedeleer, H. de, 1932, Recherches sur les pseudopodes des Rhizopodes Testacés. Les concepts pseudopodes lobosa, filosa et granulo-reticulosa: Archives de Zoologie expérimentale et générale, v. 74, no 30, p. 597-626.

Saedeleer,, H. de, 1934, Beitrag zur Kenntnis der Rhizopoden: morphologische und systematische Untersuchungen und ein Klassifikationsversuch: Mémoires du Musée Royal d'Histoire Naturelle de Belgique, no 60, p. 1-128, pls. 1-8.

Samsonov, N. A., 1908, Zur Kenntniss des Planktons des Spankansees: Sitzungsberichte der natur...Gesellschaft...Jurjew, v. 17, no 2, p. 1-92.

Sand, R., 1901, Étude monographique sur le groupe des Infusoires tentaculiféres: p. (?), Brussels

Sawyer, T. K., 1975, Marine amoebae from surface waters of Chincoteague Bay, Virginia: one new genus and eleven new species within the families Thecamoebidae and Hyalodiscidae: Transactions of the American Microscopical Society, v. 94, no 3, p. 305-323.

Schafer, C.T., Smith, J.N., Loring, D.H., 1980, Recent sedimentation events at the head of Saguenay Fjord, Canada.: Environmental Geology and Water Sciences, v. 3, 3pp. 139-150.

Schaudinn, F., 1894, Über die systematische Stellung und Fortpflanzung von Yalopus (Gromia dujardinii Schultze): Sitzungsberichte der Gesellschaft der naturforschenden Freunde zu Berlin, p. 14-22. (Reprinted as: Schaudinn, 1911a). 
Schaudinn, F., 1898, Rhizopoda Ost-Afrikas: in: "Deutsch-Ost Afrika", v. 4: "Die Thierwelt Deutsch-Ostafriks und der Nachbargebeite. Wirbellose Thiere": part 18, p. 1-13, pl. I.

Schaudinn, F., 1903, Untersuchungen über die Fortpflanzung einiger Rhizopoden. Vorl..ufige Mitteilung: Arbeiten der Kaiserlichen Gesundheitsantess, v. 19, p. 547576. (Reprinted as: Schaudinn, 1911b).

Schaudinn, F., 1911a, Über die systematische Stellung und Fortpflanzung von Yalopus (Gromia dujardini Schultze: in: Schaudinn, F., "Arbeiten": p. 45-49, Leopold Voss; Hamburg/Leipzig. - Reprinted from: Schaudinn, 1894).

Schaudinn, F., 1911b, Untersuchungen über die Fortpflanzung einiger Rhizopoden. Vorlufige Mitteilung: in: Schaudinn, F., "Arbeiten", mit nachtrag bei Hartmann, M.J; p. 521-528, Leopold Voss; Hamburg/Leipzig. - Reprinted from: Schaudinn, 1903).

Scheffelt, E., 1919, Die aquatile Fauna des Nonnenmattweihers: Mitteilungen bad....Landesver...Naturde...Natursch...Freiburg, i... Br...,v. I, p. ..

Schewiakoff, W., 1893a, Title (?): Biologisches Centralblatt, v. 8, p. 272-274.

Schewiakoff, W., 1893b, Über die geographische Verbreitung der Süsswasser Protozoen: Mémoires de L'Académie Impériale des Sciences de St.Pétersbourg, ser.7, v. 41, no 8, p. 1-201, pls. 1-4, 1 map.

Schlumberger, P., 1845. Observations sur quelques nouvelles espéces d'Infusoires de la famille des Rhizopodes: Annales des Sciences Naturelles, Zoologie, ser. 3, v. 3, p. 254-256.

Schmarda, L. C., 1846, Kleine beiträge zur naturgeschichte der Infusorien: p. (?), (Wien) (?)

Schmarda, L. C., 1854, Zur Naturgeschichte Aegyptens: Denkschriften der Kaiserlichen Akademie der Wissenschaften zu Wien (mathematischenaturwissenschaftliche Klasse), v. 7, no 2, p. 1-28, pls. 1-7.

Schmarda, L. C., 1871, Zoologie. II Aufgabe. Band I: Wilhelm Braumüller (Wien), p. i$x+1-372$.

Schmassman, W., 1924, Die Bodenfauna hochalpiner sees: Archiv für Hydrobiologie, suppl. to v. 3, p. 1-106.

Schmidt, H., 1913, Faunistische und entwicklungsgeschichtliche Studien an Sarcodinen der Umgegend von Bonn: Archiv für Protistenkunde, v. 29, no 2, p. 203247, pls. 5-6.

Schmidt, H., 1926, Untersuchungen an Rhizopoden aus Buchenhohlen: Verhandlungen naturk...Ver... Rheinlande, v. 82, p. 218-226. 
Schneider, A., 1854, Beiträge zur Naturgeschichte der Infusorien: Archiv für Anatomie, Physiologie und wissenschaftliche Medicin (Berlin), v. 21, p. 191-207, 1 pl.

Schneider, G., 1908, Der Obersee bei Reval: Archiv für Biontologie (?), v. 2, no1, p. 1-192.

Schneider, H., 1975, Die Schalenamöbe Arcella: Mikrokosmos, v. 64,no 1), p. 381384.

Schneider, R., 1886, Amphibisches Leben in den Rhizomorphen bei Burgk: Sitzsungsberichte der Königlichen Preussischen Akademie der Wissenschaften zu Berlin, p. 883-900, pl. 7.

Schoch, G., 1868, Die mikroskopischen Thiere des Süsswasser-Aquariums. I. Die Urthiere:.p. (?), Leipzig.

Schönborn, W., 1962a, Über Planktismus und Zyklomorphose bei Diffluqia limnetica (Levander) Penard: Limnologica, v. I, no I, p. 21-34.

Schönborn, W., 1962b, Neue Testaceen aus dem Grossen Stechlinsee und dessen Umgebung: Limnologica, v. I, no I, p. 83-91.

Schönborn, W., 1962c, Die Ökologie der Testaceen im oligotrophen See, dargestellt am Beispiel des Grossen Stechlinsees: Limnologica, v. 1, no 2, p. 111-182.

Schönborn, W., 1962d, Zur Ökologie der sphagnikolen, bryokolen und terrikolen Testaceen: Limnologica, v. 1, no 3, p. 231-254.

Schönborn, W., 1963, Die Stratigraphie lebender Testaceen im Sphagnetum der Hochmoore: Limnologica, v. 1, no 4, p. 315-321.

Schönborn, W., 1964a, Bodenbewohnende Testaceen aus Deutschland. I. Untersuchungen im Naturschutzgebiet Serrahn (Mecklenburg): Limnologica, v. 2, no 1, p. 105-122, pls. 1-3.

Schönborn, W., 1964b, Lebensformtypen und Lebensraumwechsel der Testaceen: Limnologica, v. 2, no 3, p. 321-335.

Schönborn, W., 1964c, Bodenbewohnende Testaceen aus Deutschland. II. Untersuchungen in der Umgebung des Grossen Stechlinsees (Brandenburg):

Limnologica, v. 2, no 4, p. 491-499.

Schönbor n, W., 1965a, Untersuchungen über die Zoochlorellen-Symbiose der Hochmoor-Testaceen: Limnologica, v. 3, no 2, p. 173-176.

Schönborn, W., 1965b, Beobachtungen an der Zellteilung von Paraquadrula (Testacea): Limnologica, v. 3, no 2, p. 235-238. 
Schönborn, W., 1965c, Neue Testaceen aus Wasserleitungen im Bezirk Halle (Saale): Limnologica, v. 3, no 3, p. 461-464.

Schönborn, W., 1965d, Studien über die Gattung Difflugiella Cash (Rhizopoda, Testacea): Limnologica, v. 3, no 3, p. 315-328.

Schönborn, W., 1965e, Die Sedimentbewohnenden Testaceen einiger Masurischer Seen: Acta Protozoologica (Warsaw), v. 3, fasc. 27, p. 297-309.

Schönborn, W., 1966a, Untersuchungen über die Testaceen Schwedisch-Lapplands. Ein Beitrag zur Systematik und Ökologie der beschalten Rhizopoden: Limnologica, v. 4, no 3, p. 517-559.

Schönborn, W., 1966c, Beitrag zur Ökologie und Systematik der Testaceen Spitzbergens: Limnologica, v. 4, no 3, p. 463-470.

Schönborn, W., 1966d, Neue Ergebnisse zur Ökologie der Testaceen: Monatsberichte der Deutschen Akademie der Wissenschaften zu Berlin, v. 8, no 5, p. 393-395.

Schönborn, W., 1966e, Beschalte Amöben (Testacean): Wittenberg-Lutherstadt: Ziemsen: "Die Neue Brehm-Bücherei", no 357, 112 pp.

Schönborn, W., 1967, Taxozönotik der beschalten Süsswasser-Rhizopoden. Eine raumstrukturanalytische Untersuchung über Lebensraumerweiterung und Evolution bei der Mikrofauna: Limnologica, v. 5, no 2, p. 159-207.

Schönborn, W., 1968a, Vergleich der zönotischen Grössen, der Verteilungsmuster und der Anpassungsstandards der Testaceen-Taxozönosen in der Biotopreihe vom Aufwuchs bis zum Erdboden: Limnologica, v. 6, no 1, p. 1-22.

Schönborn, W., 1968b, Taxonomisch-faunistische Probvleme bei der Besiedlung des Bodens an Hand der beschalten Amöben (Testacea): Pedobiologia, v. 8, p. 23-29.

Schönborn, W., 1968c, Allgemeine Probleme der Besiedlung mikromorphologischer Biotopstrukturen auf Grund der Untersuchungen an Testaceen: Biologische Rundschau (Jena), v. 6, no 5, p. 193-203.

Schönborn, W., 1973a, Paläolimnologische Studien an Testaceen aus Bohrkernen des Latnjajaure (Abisko-Gebiet; Schwedisch-Lappland): Hydrobiologia, v. 42, no 1, p. 6375 .

Schönborn, W., 1973b, Humusform und Testaceen-Besatz: Pedobiologia, v. 13, p. 353-360.

Schönborn, W., 1974, Phylogenese von Lebensgemeinschaften (Zoozönosen): Biologische Rundschau (Jena),v. 12, no 3, p. 180-195.

Schönborn, W., 1975a, Studien über die Testaceenbesiedlung der Seen und Tümpel 
des Abisko-Gebietes (Schwedisch-Lappland): Hydrobiologia, v. 46, no 1, p. 115-139.

Schönborn, W., 1975b, Ermittlung der Jahresproduktion von Boden-Protozoen. I. Euglyphidae (Rhizopoda Testacea): Pedobiologia, v. 15, no 6, p. 415-424.

Schönborn, W., 1977, Production studies on Protozoa: Oecologia, v. 27, no 2, p. 171184.

Schönborn, W., 1978, Untersuchungen zur Produktion der Boden-Testaceen:

Pedobiologia, v. 18, no 5-6, p. 373-377.

Schönborn, W., 1981a, Populationsdynamik und Produktion der Testaceen (Protozoa Rhizopoda) in der Saale: Zoologische Jahrbuch (Systematik), v. 108, p. 301-313.

Schönborn, W., 1981b, A History of Rhizopods-Colonization of Great Woryty Lake in: Dabrowiego, J. (ed.): Woryty. Studium archeologiczno-przyrodnicze zespou osadniczego kultury uzyckie: Polska Akademia Nak (?) - Polskie Bod. Archeol.(?), (Warszawa), v. 20, p. 22-24.

Schönborn, W., 1981c, Annual production and population dynamics of Testacea in soils: in: Dryl, S., Kazubski, S.L., and Ploszaj, J. (eds.): "Progress in Protozoology." Abstracts of papers submitted to VI International Congress of Protozoology, Warszawa, Poland, July, 1981: 329 pp.

Schönborn, W., 1982a, Estimation of annual production of Testacea (Protozoa) in mull and moder: Akademie der Wissenschaften der D.D.R., Zentralinstitut für Mikrobiologie und Experimentelle Therapie (Jena); Abteilung Limnologie, Pedobiologie, v. 32, p. 383393.

Schönborn, W., 1982b, Die Produktion an Gehäuse-Protozoen in der IIm:Limnologica, v. 14 , no 2 , p. 347-352.

Schönborn, W., 1983a, Modifikabilitat und Evolutionstrends bei Protozoen: Biologische Rundschau, v. 21, p. 225-235.

Schönborn, W., 1983b, Licht- und Rasterelektronenmikroskopische untersuchungen zur Schalenmorphologie und Rassenbildung Bodenbewohnender Testaceen (Protozoa Rhizopoda) sowie Vorschlage zur biometrischen Characterisierung von Testaceen shalen: Protistologica, v. 19, p. 553-566.

Schönborn, W., 1984, Studies on remains of Testacea in cores of the Great Woryty Lake (N.E.Poland): Limnologica, v. 16, no 1, p. 185-190.

Schönborn, W., 1990, Analyse subfossiler Protozoenschalen der Sedimente eines Kleinen sauren Waldsees (Kleiner Barsch-See, noerdliche DDR): Limnologica, v. 21, pp. 137-145

Schönborn, W. (1990) Shell polymorphism and habitat structure in testacea 
(Rhizopoda). Journal of Protozoology, 37 62A.

Schönborn, W. (1992) Adaptive polymorphism in soil inhabiting testate amoebae (Rhizopoda): Its importance for delimitation and evolution of asexual species. Archiv für Protistenkunde, 142 139-155.

Schönborn, W. (1992) The role of protozoan communities in freshwater and soil ecosystems. Acta Protozoologica, 31 11-18.

Schönborn, W. (1992) Comparative studies on the production biology of protozoan communities in freshwater ecosystems. Archiv für Protistenkunde, 141 187-214.

Schönborn, W., Petz, W., Wanner, M. \& Foissner, W. (1987) Observations on the morphology and ecology of the soil-inhabiting testate amoeba Schoenbornia humicola (Schönborn, 1964) Decloitre, 1964 (Protozoa: Rhizopoda). Archiv für Protistenkunde, 134 315-330.

Schönborn, W. \& Peschke, T. (1990) Evolutionary studies on the AssulinaValkanoria complex (Rhizopoda: Testaceafiliosea) in Sphagnum and soils. Biology and Fertility of Soils, 9 95-100.

Schönborn, W., Flössner, D., Proft, G., 1965, Die Limnologische Characterisierung des Profundals einiger norddeutscher Seen mit Hilfe von Testaceen-Gemeinschaften: Limnologica, v. 3, no 3, p. 371-380.

Schönborn, W., Flössner, D., Proft, G., 1966, Die Limnologische Characterisierung des Profundals einiger norddeutscher Seen mit Hilfe von Testaceen-Gemeinschaften: Verhandlungen des Internationalen Vereins für Limnologie, v. 16, p. 251-257.

Schönborn. W., 1966b, Testaceen als Bioindikatoren im System der Seentypen. Untersuchungen in masurischen Seen und im Suwalki-Gebiet (Polen): Limnologica, v. 4, no 1, p. 1-11.

Schouteden, H., 1906, Les Rhizopodes testacés d'eau douce, d'aprés la Monographie du Prof. A. (sic) Awerintzew: Annales de Biologie Lacustre, v. 1, no 3, p. 327-382, 2 pls.

Schrank Paula, F. von, 1802, Briefe naturhistorischen, physikalischen und ökonomischen Inhaltes an Herrn Bernhard Sebastian Nau... Nebst drey vorausgeschickten naturhistorischen Abhandlungen; J.C. Schubart, p. i-viii + 1384, 4.pls. (Erlangen).

Schultze, F. E., 1874a, Rhizopodenstudien. I.: Archiv für mikroskopische Anatomie, v. 10, p. 328-350, pl. 22.

Schultze, F. E., 1874b, Rhizopodenstudien. II.: Archiv für mikroskopische Anatomie, v. 10 , p. $377-400$, pls. $26-27$. 
Schultze, F. E., 1875a, Rhizopodenstudien. III.: Archiv für mikroskopische Anatomie, v. II, p. 94-139, pls. 5-7.

Schultze, F. E., 1875b, Rhizopodenstudien. IV.: Archiv für mikroskopische Anatomie, v. 11, p. 329-353, pls. 18-19.

Schultze, F. E., 1875c, Rhizopoden: in:"Zoologische Ergebnisse der Nord-Seefahrt vom 21 Luli bius 9 September 1872" (series of articles), Jahresbericht der Kommission zur wissenschaftlichen Untersuchung der deutschen Meere in Kiel, v. 1, no 2-3, p. 97-114, pl. 2.

Schultze, F. E., 1875c, Rhizopodenstudien. V.: Archiv für mikroskopische Anatomie, v. II, p. 583-596, pls. 25-26.

Schultze, F. E., 1877, Rhizopodenstudien. VI.: Archiv für mikroskopische Anatomie, v. 13 , p. $9-30$, pls. $2-3$.

Schultze, M. S., 1854, Über der Polythalamien (Foraminiferen) nebst Benerkungen über die Rhizopoden im Allgemeinen: Wilhelm Engelmann, folio, p. i-xviii + 1-68, pls. 17. (Leipzig). [what should be bold?]

Schultze, M., 1860, Die Gattung Cornuspira unter den Monothalamien, und Bemerkungen über die Organisation und Firtpflanzung der Polythalamien: Archiv für Naturgeschichte, v. 26, p. 287-310. (Translated to English in: Schultze, 1861).

Schultze, M., 1861, On the genus Coprnuspira, belonging to the Monothalamia; with remarks on the organization and reproduction of the Polythalamia: Annals and Magazine of Natural History, ser.3, v. 7, no 4, p. 306-321.(Translation from: Schultze 1860).

Schultze, M., 1863, Das Protoplasma der Rhizopoden der Pflanzenzellen. Ein Beitrag zur Theorie der Zelle: Engelmann; p. i-iv + 1-68 (Leipzig).

Schweigger, A.F., 1819, Beobachtungen auf naturhistorischen Reisen von August Friedrich Schweigger. Anatomish-physiologische Untersuchungen über Corallen; nebst einem Anhange, Bemerkungen über den Bernstein enthaltend: Georg Reimer; p. i-vi + 1-127, 12 tabs., 8 pls. (Berlin). 


\section{SCO-SYV}

Scott, D. B., Medioli, F. S., Schafer, C. T., 1977, Temporal changes in foraminiferal distributions in Miramichi River estuary, New Brunswick: Canadian Journal of Earth Sciences, v. 14, no 7, p. 1566-1587.

Scott, D. B., Schafer, C. T., Medioli, F. S., 1980, Eastern Canadian estuarine Foraminifera: a framework for comparison: Journal of Foraminiferal Research, v. 10, no 3, p. 205-234.

Scott, D.B., Medioli, B.E., Waringer, J., Medioli, F.S., Wightman, W.G., Thibeaudeau, S., 1995, Salt marsh foraminifera and arcellaceans, keys to determination of marine vs. freshwater peat/ lignite/ coal environments: Abstract, Geological Society of America, v. 27, pp. 31

Scott, D.B., Medioli, F. S., 1980, Post-glacial emergence curves in the Maritimes determined from marine sediments in raised basins: Proceedings of Coastlines '80, published by National Science and Engineering Research Council, p. 428-449

Scott, D.B., Medioli, F. S., 1983, Agglutinated Rhizopods in Lake Erie:Modern distribution and stratigraphic implications: Journal of Paleontology, v. 57, no 4, p. 809820.

Scott, D.B., Medioli, F. S., 1984, Measured vs. theoretical sea-level changes in Atlantic Canada: Proceedings International Symposium on Late Quaternary Sea-Level Changes and Coastal Evolution, Mar del Plata, Argentina, p. 89-91.

Scott, D.B., Medioli, F. S., 1987, Holocene sea levels, paleoceanography, and late glacial ice configuration near the Northumberland Strait, Maritime Provinces: Canadian Journal of Earth Science, v. 24, p. 668-675.

Scott, D.B., Suter, J.R,, Kosters, E.C , 1991, : Marsh foraminifera and arcellaceans of the lower Mississippi Delta; controls on spatial distributions: Micropaleontology, v. 37, pp. 373-392

Scourfield, D. J., 1897, Contributions to the non-marine fauna of Spitzbergen. I. Preliminary note and reports on the Rhizopoda: Proceedings of the Zoological Society of London, p. 784-792, pl. 45.

Seis, J., 1971, Rhizopodenanalytische untersuchungen an den mooren des pleistozaenen Salzachvorlandgletschers, Vorbericht, Leopoldskroner Moor: Berichte aus dem Haus der Natur in Salzburg, Abteilung B, Geologisch-Mineralogische Sammlungen, v. 2, pp. 10-14.

Seis, J.,year (?), Investigations of Rhizopoda in the Postglacial Lakes of the Pleistocene glaciers of the Salzach Foreland; preliminary report: Leopoldskron Moor: Haus...Nat... Ber... ABt.... B...., Geol....-Mineral..., Samml....., Salzburg, v. 2, p. 10-14. 
Senaud, J., Pussard, M., Pons, R., 1976, Particularite ultrastructurales de Gocevia fonbrunei Pussard 1965: Journal of Protozoology, v. 23(4), p. 20A.(?)

Simitzis, A.-M., Legoff, F., 1981, Observations on testate amoebae of the group Capsellina Penard 1909 Rhogostoma Belar 1921 (Gromiidae, Gromiida, Filosea): Protistologica, v. 17, no 1, p. 99-111.

Skorikov, A. S., 1907, Quelques faits concernant la biologie d'un etang dans le jardin de la Tauride a St. Petersbourg: Bulletin de l'Academie des Sciencea (St. Petersburg), v. (?), p. 119-126.

Skorikov, A. S., 1907, Quelques faits concernant la biologie d'un etang dans le jardin de la Tauride a St. Petersburg: Bull. Acad. Sci. (St. Petersb.), p. 119-126.

Sladecek, V., 1951, Le zooplancton de 1'etang-lac de Macha et deux autres etangs voisins (Bohme du Nord): Casopis Narodniho Muzea. Oddil Priroddovedny, v. 120, p. 29-34.

Sleigh, M., 1973, The biology of Protozoa: p. I-VIII, 1-315; Edward Arnold (London) and American Elsevier (New York).

Sleigh, M.A. (1989) Protozoa and other protists. 4th Edition. Edward Arnold, London.

Smagowicz K., 1976, On the zooplankton of Lake Zeribar, western Iran: Acta Hydrobiologica (Kraków), v. 18, no 1, p. 89-100.

Smagowicz, K., 1975, Meiofauna of riverine waters of the Biatka Tatrzaka. 1. Testaceous Rhizopoda (Testacea): Acta Hydrobiologica (Kraków), v. 17, no 4, p. 405-414.

Smirnov, A.V., Goodkov, A.V., 1994, Freshwater gymnamoebae with a new type of surface structure Paradermamoeba valamo and P. levis sp. n. (Thecamoebidae), and notes on the diagnosis of the family: Acta Protozoologica, v. 33, pp. 109-115.

Smith, H.G. (1986) The testate amoebae rhizopod fauna of Drepanocladus moss carpet near Rothera Station, Adelaide Island. British Antarctic Survey Bulletin No. 72, 77-79.

Smith, H.G. (1987) A species-poor testate rhizopod fauna on Brabant Island. British Antarctic Survey Bulletin No. 77, 173-176.

Smith, H.G. (1992) Distribution and ecology of the testate rhizopod fauna of the continental Antarctic zone. Polar Biology, 12 629-634.

Smith, H.G. and Wilkinson, D.M. (1986) Biogeography of testate rhizopods in the southern temperate and Antarctic zone. Colloque les Écosystemes Terrestres Subantarctiques, 58 83-96.

Smith, H. G., 1973a, The Signy Island terrestrial reference sites: II. The Protozoa: 
British Antarctic Survey Bulletin, no 33, 34, p. 83-87.

Smith, H. G., 1973b, The Signy Island terrestrial reference sites: III. Population ecology of Corythion dubium (Rhizopoda: Testacida) in site 1: British Antarctic Survey Bulletin, no 33, 34, p. 123-135.

Smith, H. G., 1973c, The terrestrial Protozoa of Elephant Island, South Shetland Islands: British Antarctic Survey Bulletin, no 31, p. 55-62.

Smith, H. G., 1974, The ecology of Protozoa in chinstrap penguin guano: British Antarctic Survey Bulletin, no 35, p. 33-50.

Smith, H. G., 1974a, The ecology of Protozoa in chinstrap penguin guano: British Antarctic Survey Bulletin, no 35, p. 33-50.

Smith, H. G., 1974b, A comparative study of Protozoa inhabiting Drepanocladus moss carpet in the South Orkney Islands: British Antarctic Survey Bulletin, no 38, p. 1-16.

Smith, H. G., 1975, Protozoaires terricoles de 1'lle de la Possession: Revue d'Ecologie et de Biologie du Sol, v. 12, no 2, p. (?)

Smith, H. G., 1975, Protozoaires terricoles de 1'lle de la Possession: Revue d'Ecologie et de Biologie du Sol, v. 12, no 2, pages unknown

Smith, H. G., Hardy, P., Leith, I. M., Spaull, V. W., Twelves, E. L., 1974, A biological survey of St. Paul's Rocks in the equatorial Atlantic Ocean: Biological Journal of the Linnean Society (London), v. 6, no 2, p. 89-96.

Smith, H. G., Hardy, P., Leith, I. M., Spaull, V. W., Twelves, E. L., 1974, A biological survey of St. Paul's Rocks in the equatorial Atlantic Ocean: Biological Journal of the Linnean Society (London), v. 6, no 2, p. 89-96.

Song, H. H., 1982, Limno-biological investigation of Lake Ok-Jeong: BULL.-KOREANFISH.-SOC., v. 15, pp. 1-25 (in Korean)

Spandel, E., 1909, Der Rupelton des Mainzer Beckens, seine Abteilungen und deren Foraminiferenfauna, sowie einige weitere geologishpaläontologishen Mitteilungen über das Mainzer Becken: Bericht über die Thätigkeit des Offenbacher Vereins für Naturkunde, no 43-50, p. 57-230, pls. 1-2.

Stehle, M. E., 1920, A preliminary survey of the Protozoa of Mirror Lake, on the Ohio State University Campus: Ohio Journal of Science, v. 20, no 4, p. 89-126.

Stehle, M. E., 1923, Surface plankton Protozoa from Lake Erie in the Put-in-Bay region: Ohio Journal of Science, v. 23, no 1, p. 41-54.

Stein, S. F. N. von, 1859, Über die inm aus eigener Untersuchung bekannt gewordenen Süsswasser-Rhizopoden: Abh. K. Böhm. Gesellsch. Wiss., 5. Folge 10, p. 41-43. 
Steinecke, F., 1914, Die beschalten Wurzelfüssler (Rhizopoda: testacea) des Zehlaubruches: Schriften der Physikalisch-Ökonomischen Gesellschaft zu Könisberg, v. 54, p. 299-328.

Steinecke, F., 1927, - In Library (QH 301 I 624), get title and read - [in: Gams, H., "Die Geschichte der Lunzer Seen, Moore und W\{lder"]: Internationale Revue der gesamten Hydrobiologie, v. 18, p. (?).

Steinecke, F., 1927a, Leitformen und Leitfossilien des Zehlaubruches: Botanische Archiv; zeitschrift für die gesamte Botanik (Koenigsberg): v. 19, p. 328-343.

Steinecke, F., 1929, Die Nekroznose des Zehlaubruches: Schriften der Physikalischkonomischen Gesellschaft zu Knigsberg, p. (?)

Steinmann, P., 1907, Die Tierwelt der Gebirgsbache: Archiv für Hydrobiologie, v. 3, p. 266-273.

Stenroos, K. E., 1898, Das Thierleben im Nurmijärvi-See. Eine faunistische-biologische Studie: Acta Societatis Flora et Fauna Finnica, v. 17, no 1, p. 1-259, pls. 1-3.

Stepanek, M., 1952a, Testacea of the pond of Hr\{dek at Kunratice (Prague): Acta Musei Nationalis Pragae [Sbornk N\{rodniho v Praze], ser.b, v. 8, p. 1-55.

Stepanek, M., 1952b, Menavky a slunivky tunky Hr\{dku u Kunratic: Casopis N\{rodniho Musea, v. 121 , p. $76-80$.

Stepanek, M., 1953, Rhizopoda jako biologicke indikatory znecisteni vod:

Prirodovedne sbornik Ostravskeho kraje, v. 14, p. 470-505.

Stepanek, M., 1963, Die Rhizopoden aus Katanga (Kongo-Africa): Annales Mus. Roy. Afrique centr. (Tervueren), v. 117, p. 9-91.

Stepanek, M., 1967, Testacea des Benthos der Talsperre Vranov am Thayafluss: Hydrobiologia, v. 29, p. 1-66.

Stepanek, M., 1968, Die Rhizopoden des Tiefenschlammes in Bodensee: Archiv für Hydrobiologie, supplement v. 33 (Falkau Arbeiten 6), p. 442-450.

Stepanek, M., Jiri, J., 1958, Difflugia gramen Penard, Difflugia gramen var. achlora Penard and Difflugia gramen f. globulosa f.n. (Morphometrical and statistical study): Hydrobiologia, v. 10, p. 138-156.

Stiller, J., 1957, Zur Biologie und verbreitung der Protozoen- und crustaceenfauna eines Mittelgebirgsbaches in Ungarn: Archiv für Hydrobiologie, v. 53, p. 392-424.

Stokes, A.C., 1882, A food-habit of Difflugia pyriformis: American Microscopical Journal, v. 3, p. 93.

Stout, J. D., 1963, The distribution of rhizopod and ciliate Protozoa in the soils, forest 
litter and peats of the New Zealand area: in: Ludvík, J., Lom. J. and Vávra, J., (eds.): "Progress in Protozoology. Proceedings of the First International Congress on Protozoology held in Prague, August 22-31, 1961", p. 334; Publishing House of the Czechoslovak Academy of Sciences (Prague).

Stout, J. D., 1973a, The ecology of New Zealand snow-tussock grasslands: in: DePuytorac, P. and Grain, J., (eds.): "Progress in Protozoology. Abstracts of papers read at the Fourth International Congress on Protozoology, Clermont-Ferrand, 210 Septembre 1973": p. 404; Université de Clermont (Clermont-Ferrand).

Stout, J. D., 1973b, The ecology of New Zealand thermal pools: in: DePuytorac, P. and Grain, J., (eds.): "Progress in Protozoology. Abstracts of papers read at the Fourth International Congress on Protozoology, Clermont-Ferrand, 2-10 Septembre 1973": p. 405; Université de Clermont (Clermont-Ferrand).

Stout, J. D., Heal, O. W., 1967, Protozoa: in: Burgess, A. and Raw, F., (eds.): "Soil Biology", p. 149-195; Academic Press (London).

Stout, J. D., Walker, G. D., 1976, Discrimination of mineral particles in test formation by Thecamoebae: Transactions of the American Microscopical Society, v. 95, no 3, p. 486-489.

Strasburger, E., 1876, Studien über Protoplasma: Jenaische Zeitschrift für Medizin und Naturwissenschaft, v. 10, p. 395-446, pls. 13,14.

Studer, T., 1893, Faune du Lac de Champex, Canton du Valais: (Bibliothèque universelle), Archives des Sciences physiques et Naturelles, ser.3, v. 30, p. 637645.

Stump, A. B., 1935, Observations on the feeding of Difflugia, Pontigulasia and Lesquereusia: Biological Bulletin, v. 69, no 1, p. 136-142.

Stump, A. B., 1936, The influence of test materials on reproduction in Pontigulasia vas (Leidy) Schouteden: Biological Bulletin, v. 70, no 1, p. 142-147.

Stump, A. B., 1937, Methods of culturing Testacea: in: Galstoff, P. S., Lutz, F. E., Welch, P. S. and Needham, J. G., (eds.): "Culture methods for invertebrate animals", p. 92-93; Comstock Publishing Co. Inc. (Ithaca, N.Y.).

Stump, A. B., 1943, Mitosis and cell division in Pontigulasia vas (Leidy) Schouteden: Journal of the Elisha Mitchell Scientific Society, v. 59, no 1, p. 14-23, pl. 1.

Stump, A. B., 1959, Mitosis in the rhizopod Lesquereusia spiralis: Journal of Protozoology, v. 6, no 2, p. 185-189.

Stump, A. B., 1963, Interphase changes in the nucleus of Lesquereusia spiralis (Ehrenberg) Penard: Bulletin of the South Carolina Academy of Science, v. 25, p. 15. 
Stump, A. B., 1965, Asexuality and sub-speciation in Lesquereusia spiralis as shown in long term pure line cultures: Bulletin of the South Carolina Academy of Science, v. 27, p.43-44.

Stump, A. B., 1971, Observations on some internal structures and pseudopods of Lesquereusia spiralis (Rhizopoda, Testacida): Journal of Protozoology, v. 18, no 3, p. 417-419.

Stump, A. B., Freeman, C. J. jr., 1967, A "window" method for the observation of Lesquereusia spiralis: Journal of Protozoology, v. 14 (supplement), p.39.

Sudzuki, M. 1976a, Protozoans in the marine beach interstices. I. Fauna and its distribution around Kasado Island in Seto Inland Sea: Japanese Journal of Protozoology, v. 9, p. 8-9.

Sudzuki, M. 1976b, Microscopical marine animals scarcely known from Japan. I. Microand meio-faunae around Kasado Island in the Seto Inland Sea of Japan: Proceedings of the Japanese Society of Systematic Zoology, v. 12, p. 5-12.

Sudzuki, M. 1977a, Protozoans in the marine beach interstices. II. Taxonomy and ecology of Testacea from a sandy island recently constructed: Japanese Journal of Protozoolooy, v. 10, p. 23.

Sudzuki, M. 1978a, The effects of excrements of five domestic animals on the individual density and faunistic component of soil microbiota in soil: Japanese Journal of Protozoology, v. II, p. 6-7.

Sudzuki, M. 1978b, Protozoans in the marine beach interstices. III. Psammobiont protozoans from Niijima Island, Tokyo: Japanese Journal of Protozoology, v. II, p. 8.

Sudzuki, M. 1979a, Psammobiont Rhizopoda and Actinopoda from marine beaches of Japan: Acta Protozoologica, v. 18, no 2, p. 293-304, pls. 1-8.

Sudzuki, M. 1979b, Marine interstitial Testacea from Plau Pinang, Malaysia: Annot...zool...japon... v. 52, no 1, p. 50-53.

Sudzuki, M. 1979c, Some aspects of the haline interstitial biota from Ryukyu Shot, subtropical chain islands, Southwest Japan: Sesoko Marine Science Laboratory, Technical Report no 6, p. 37-50, pls. 1-4.

Sudzuki, M. 1983a, Protozoans in the marine beach interstices. IX. Psammobiont Testacea from Abidjan, Côte d'Ivoire, West Africa: Japanese Journal of Protozoology, v. 16 , no 1 , p. (?).

Sudzuki, M., 1977b, Recent portraits of wild biota in Japan. III. A man-made sandy beach constructed in the Bay of Tokyo: Obuhrohso (Nihon Daigaku), v. 8, p. 221-239.

Sudzuki, M., 1978c, Some approaches to the estimation of the biomass for microfauna 
communities. II. Differences in the occurrences of microbiota inhabiting litters, mosses, especially soils from four terrestrial ecosystems, Environmental Agency (Tokyo), p. 181-215.

Sudzuki, M., 1979d, Marine Testacea of Japan: Sesoko Marine Science Laboratory, Technical Report no 6, p. 51-61, pls. I-IV.

Sudzuki, M., 1983b, Incertae Sedis. I. Strange animalcules from a garden pond in Penang, Malasia: Obuhrohso (Nihon Daigaku), v. 15, p. 173-176.

Suxena, M. R., 1979, Algae and Testacea from high altitudes of Himalayas. I (collected by N. C. C. Punch Chulli Expedition, W. Himalaya 1970): Hydrobiologia, v. 65, no 2, p. 107-128.

Swarczewsky, B., 1908, Über die Fortpflanzungserscheinungen bei Arcella vulgaris Ehr.: Archiv für Protistenkunde, v. 12, no 3, p.173-212, pls. 14-16.

Syvitski, J.P.M., Schafer, C.T., 1996, Evidence for an earthquake-triggered basin collapse in Saguenay Fjord, Canada: Sedimentary Geology. v. 104, pp. 127-153: In: Marine sedimentary events and their records: Shiki, T., Chough, S.K. and Einsele, G.(eds.), Elsevier. Amsterdam, Netherlands. 


\section{TAP-WAI}

Tappan, H., 1966, Valkanova, a new name for the thecamoebian Euglyphella Valkanov, 1962 non Warthin, 1934 (Protozoa: Rhizopodea): Proceedings of the Biological Society of Washington, v. 79, p. 89.

Taranek, K. J., 1881, Beiträge zur Kenntniss der Süsswasser-Rhizopoden Böhmens: Sitzungsberichte der Königlichen Böhmischen Gesellschaft der Wissenschaften , p. $220-235$.

Taranek, K. J., 1882, Monographie der Böhmen's. Ein Beitrag zur Kenntniss der Süsswasser-Monothalamien: Abhandlungen der Königlichen Böhmischen Gesellschaft der Wissenschaften, ser. 6, v. II, mathematisch-naturwissenschaftliche Classe, no 8, p. I-IV, 1-56, pl. 1-5.

Tarnogradskii, D.A., 1957, - -Russian title (?)- Microflora and microfauna of peats in the Caucasus. 6: The Dedvorakski sphagnum bog: Raboty sev. kavk. gidrobiol. Sta. Gorsk. Sel'khoz. Inst., v.6(1-2), p.3-56, 3 pls.

Tarnogradskii, D. A., 1959, -Russian title (?) - Microflora and microfauna of peats in the Caucasus. 8. A sedge-sphagnum lake in the upper reaches of the Balkarian river Cherek: Raboty sev. kavk. gidrobiol. Sta. Gorsk. Sel'khoz. Inst., v. 6, no 3, p. 3-59, 8 plates.

Tarnogradskii, D. A., 1961, -Russian title (?) - Microflora and microfauna of peats in the Caucasus. 5. The Sphagnetums of Makharsky Gorge (Karatschaevo-Circassian Republic): Raboty sev. kavk. gidrobiol. Sta. Gorsk. Sel'khoz. Inst., v. 7 no 1,2, p. 3-32.

Tatem, J. G., 1870, Notes on new Infusoria: Monthly Microscopical Journal, v. 4, p. 313-314, pl. 68.

Tatem, J. G., 1880, Mr. Archer's genus Hyalosphenia: Monthly Microscopical Journal, v. 17, p. 311.

Thalwitz, J., 1906, Die Tierwelt: in: Schorler, J. and Thalwitz. J., (eds.): "Pflanzen und Tierwelt des Moritzburger Grossteiches bei Dresden", Annales de Biologie lacustre, v. I, no 2, p. 256-310.

Thibaudeau, S.A., Medioli, F.S., 1986, Carboniferous thecamoebians and marsh foraminifera: new stratigraphic tools for ancient paralic deposits: Abstract: Geological Society of America, Annual Meeting, San Antonio, pp. 771.

Thibaudeau, S.A., Medioli, F.S., Scott, D.B., 1987, Carboniferous marginal-marine rhizopods, a morphological comparison with Recent correspondents: Abstract, Geological Society of America, v. 19, 7, pp. 866.

Thiébaud, M., 1906a, Sur la faune invertébrée du lac de St.Blaise: Zoologischer Anzeiger, v. 29, p. 795-801. 
Thiébaud, M., 1906b, Contribution à l'étude de la faune des eaux du Jura: Annales de Biologie lacustre, v. 1, no 1, p. 57-113.

Thiébaud, M., 1906c, Contribution à la biologie du lac St.Blaise: Annales de Biologie lacustre, v. 3, no 1, p. 54-140, pl. 1-5.

Thomas, R., 1953, Sur deux formes critiques du gendre Difflugia Leclerc: Bulletin de la Société Zoologique de France. v. 78, p. 132-136.

Thomas, R., 1954, Thécamoebiens de la région bordelaise: Bulletin de la Société d'Histoire Naturelle de Toulouse, v. 89, p. 245-264, pls. 1-4.

Thomas, R., 1958a, Observations sur le revetement des Trinema: Bulletin of Applied Microscopy, v. 8, p. 105-108.

Thomas, R., 1958b, Reflexions sur la nutrition des thécamoebiens: Travaux du Laboratoire de Botanique et Cryptogamie de la Faculté nde Medicine et Pharmacie de I'Institut de Bordeaux, v. (?), p. 1-2.

Thomas, R., 1958c, Le genre Plagiopyxis Penard: Hydrobiologia, v. 10, p. 198-214.

Thomas, R., 1960a, Essai pour un catalogue des rhizopodes testacés des biotopes terricoles: Bulletin de la Société de Pharmacie de Bordeaux, v. 99, p. 13-22.

Thomas, R., 1960b, Les Thécamoebiens muscicoles et terricoles: notions d'écologie générale et comparative: Procès-Verbaux de la Société Linneénne de Bordeaux, v. 98, p. 27-53, pls. 1-2.

Thomas, R., 1961a, Étude sur les Thécamoebiens des stations à hygrométricité faible et variable: Mémoire de Diplôme d'Études Supérieures, Faculté des Sciences de Clermont-Ferrand, p. 1-68.

Thomas, R., 1961b, Note sur quelques Rhizopodes de France: Cahiers des Naturalistes, Bulletin des Naturalistes Parisiens, new series, v. 17, p. 74-80.

Thomas, R., Chardez, D., 1958, Étude critique de Trinema penardi nom.nov. (Thécamoebiens): Cahiers des Naturalistes, Bulletin des Naturalistes Parisiens, new series, v. 14, p. 101-104.

Thomas, R., Gauthier-Lièvre, L., 1959a, Le genre Lesquereusia Schlumberger 1845 (Rhizopodes testacés): Bulletin de la Société d'Histoire Naturelle de l'Afrique du Nord, v. 50, p. 34-83, pls. 1-2.

Thomas, R., Gauthier-Lièvre, L., 1959b, Note sur quelques Euglyphidae d'Afrique: Bulletin de la Société d'Histoire Naturelle de l'Afrique du Nord, v. 50, p. 204-221.

Thomas, R., Gauthier-Lièvre, L., 1961, Addenda and corrigenda. Le genre Lesquereusia Schlumberger: Bulletin de la Société d'Histoire Naturella de l'Afrique du Nord, v. 52, p. 162-163. 
Thomas, R., Horasse, R., 1962, Sur la constitution des théques des Thécamoebiens. I.: Le genre Trinema et Trinema lineare Penard: Bulletin of Applied Microscopy, v. 12, p. 117-119.

Thomas, R., Mabille, J., 1956, Rhizopodes thécamoebiens observés dans le departement de l'Aisne: Cahiers des Naturalistes, Bulletin des Naturalistes Parisiens, new series, v. 12, p. 26-32.

Todd, R., Brönnimann, P., 1957, Recent Foraminifera and Thecamoebina from the eastern Gulf of Paria: Cushman Foundation for Foraminiferal Research, Special Publication no 3, p. 1-43, pl. 1-12.

Tolonen, K. (1966) Stratigraphic and rhizopod analyses on an old raised bog, Varrassuo, in Hollola, South Finland. Annales Botanica Fennici, 3 147-166.

Tolonen, K. (1986) Rhizopod Analysis. In: Berglund, B.E. (ed.) Handbook of Holocene palaeoecology and palaeohydrology. John Wiley \& Sons Ltd. Chichester. 645-666.

Tolonen, K., Huttunen, P., Junger, H. (1985) Regeneration of two coastal raised bogs in Eastern North America. Annales Academia Scientiarum Fennicae. III. GeologicaGeographica. 139 A. 5-51.

Tolonen K., Warner, B.G. \& Vasander, H. (1994) Ecology of testaceans (Protozoa: Rhizopoda) in mires in Southern Finland: II. Multivariate Analysis. Archiv für Protistenkunde, 144 97-112.

Tolonen, K., Warner, B.G., Vasander, H., 1992, Ecology of testaceans (Protozoa:

Rhizopoda) in mires in southern Finland: 1. Autecology: Archiv für Protistenkunde, v. 142, pp. $119-138$

Torigai, K., 1996. Distribution of recent thecamoebians in Lake Winnepeg. Unpublished honours thesis, Carleton University, Ottawa, Ontario, 57 pp.

Torres, V.S. and Jebram, D.H.A. 1993. Arcella gibbosa microsoma var. n. (Protozoa: Sarcodina, Arcellinida): Descrição e Observações feitas em seu Cultivo. Biotemas 6(2):20-29.

Torres, V.S. and Jebram, D.H.A. 1994. Amebas Testáceas Ocorrentes na Região de Porto Alegre, RS. Biotemas 7(1/2):65-78.

Torres, V.S. and Jebram, D.H.A. 1995. Caracterização Morfológica e Biométrica de 3 Amebas Testáceas Filófitas. Biotemas 8(1):30-35.

Torres, V.S. 1996. Vacúolos de Gás e Flutuação em Difflugia mitriformis Wallich, 1864 (Sarcodina: T estaceolobosea). Revista brasileira de Zoologia 13(1):67-75.

Torres, V.S. 1996. Estudos Sobre Difflugia lobostoma Leidy, 1879 (Protista: 
Rhizopoda, Testaceolobosea). Revista brasileira de Zoologia 13 (2):475-487.

Torres, V.S. 1996. Elaboração de Coleção de Culturas de Amebas Testáceas - I: Arcellidae. Biociências 4(1):53-63.

Torres, V.S. 1998. Amebas Testáceas Ocorrentes na Região de Porto Alegre, Rio Grande do Sul. II. Novos Registros para a Região. Revista brasileira de Zoologia 15(2):545-552.

Torres, V.S. 1999. Contribuição ao Conhecimento da Microbiota Aquática do Parque Nacional dos Aparados da Serra, Cambará do Sul, RS, BRASIL. Com. Mus. Ciênc. Tecnol. PUCRS ^ série Miscelânea 4: no prelo.

Toutain, F., 1981, Observations sur l'activité probable des Thécamoebiens dans l'altération cuticulaire des litiéres forestiéres: in: Alabouvette, C. et al.: "Les Protozoaires du sol: aspects écologiques et méthodologhgiques" L'Année biologique, v. 20, no 3, p. 255-303.

Tripp, H.E., 1870, Notice of recent observations on .......??...... and Monads: Proceedings, Bristol (?) Natural.Society, new series, v. 4, p. 9-22.

Vaek, M., Rika, B., 1957, Namurian Thecamoebina from the Ostrava Karvinà coal district: Sbornìk Národnìno Musea v Praze. Rada b: Pirodni Vdy (Acta Musei Nationalis Pragae), ser. b: Historia Naturalis, v. 13, no 5, p. 333-340, pls. 40-41.

Valkanov, A., 1932, Yadrenoto delenie i yadreniyat stroezh pri Cochliopodium i Cyathomonas kato osnova za razglezhdaneto na edin kariologichen problem (Die Kernteilung und Kernverhältnisse bei Cochliopodium und Cyathomonas als Grundlage zur Betrachtung eines karyologischen Problems - This is the German title of the summary): Godishnik na Sofiiskija Universitet', II, Fiziko-Matematicheski Fakultet' (Annales of the University of Sofia, II, Faculty of Physics and Mathematics), sect.I (Zoology), v. 28 no 3, p. 153-196.

Valkanov, A., 1934, Gocevia pontica Valkanov. Protistenstudien 9: Archiv für Protistenkunde, v. 83, no 2, p. 352-353.

Valkanov, A., 1938, Über die Fortpflanzung von Gromia dujardini M. Schultze. Protistenstudien 10: Archiv für Protistenkunde, v. 90(3), p. 393-395.

Valkanov, A., 1940, Die Heliozoen und Proteomyxien. Artbestand und sonstige kritische Bemerkungen: Archiv für Protistenkunde, v. 93, no 2, p. 225-254.

Valkanov, A., 1962a, Über die Kopulation der Testaceen (Rhizopoda Testacea): Doklad Bolgarskoi Akademii Nauk (Reports of the Bulgarian Academy of Science), v. 15, no 3, p. 305-308.

Valkanov, A., 1962b, Paraquadrula madarica n. sp.(Rhizopoda Testacea) und ihre Kopulation: Doklad Bolgarkoi Akademii Nauk (Reports of the Bulgarian Academy 
of Science), v. 15, no 4, p. 423-426.

Valkanov, A., 1962c, Euglvphella delicatula n. g., n. sp. (Rhizopoda Testacea ) und ihre Kopulation: Doklad Bolgarkoi Akademii Nauk (Reports of the Bulgarian Academy of Science), v. 15, no 2, p. 207-209.

Valkanov, A., 1963, Über die Kopulation bei der Gattung Phryganella (Rhizopoda Testacea): Doklad Bolgarkoi Akademii Nauk (Reports of the Bulgarian Academy of Science), v. 16, no 1, p. 97-100.

Valkanov, A., 1966a, Über die Fortpflanzung der Testaceen (Rhizopoda Testacea): Bulgarska Akademiya na naukite, Izvestiya na Zoologischeskiya Institut s Muzei (Bulgarian Academy of Science, Bulletin of the Institut and the Museum of Zoology (Sofia)), v. 22, p. 5-49, 12 unnumbered plates.

Valkanov, A., 1966b, Democystidium cochliopodii n. sp.. seine Struktur und Entwicklunq: Nova Hedwiqia. v. 12, p. 3-4.

Valkanov, A.K., 1936, Versuch einer Revision der Rhizopodengattung Cochliopodium: Sitzungsberichte des Gesellschaft des naturforschenden Freunde, p. 92-101.

Valkanov, A.K., 1942, Über Protistenstudien XI. Eine die Teilung der Testaceen begleitende Erscheinung: Godishnik na Sofiiskija Universitet', FizikoMatematicheski Fakultet' (Annales of the University of Sofia, II, Faculty of Physics and Mathematics), sect.I (Zoology), v. 38, no3, p. 31-60.

Valkanov. A., 1970. Beiträg zur Kenntniss der Protozoen des Schwarzen Meeres: Zoologischer Anzeiger, v. 184. p. 241-290.

Vanhöffen, E., 1897. Die Fauna und Flora Grönlands: in:: von Drygalski, E., (ed.): "Grönland-Expedition der Gesellschaft für Erdkunde zu Berlin 1891-1893, unter Leitung von Erich von Drygalski", v. 2, no 1, p. 1-383, pls. 1-9; W.H.Kühl (Berlin).

Van Oye, P. (1956) On the thecamoeban fauna of New Zealand with description of four new species and biogeographical discussion. Hydrobiologia 8 16-37.

Varga, L., 1960, Über die moosbewohnende Mikrofauna aus einer Dachrinne von Tihany: Annales Instituti Biologici (Tihany) Hungaricae Academiae Scientiarum, v. 27 , p. $169-182$.

Varga, L., 1963, Weitere untersuchungen über die aquatile Mikrofauna der Baradla Hohle bei Aggtelek (Ungarn): Biospeleologia Hungarica, xviii; v. 9, p. 439-458.

Vejdovsk, F., 1881, Über die Rhizopoden der Brunnenwässer von Prag: Sitzungsberichte der Königlichen Böhmischen Gesellschaft der Wissenschaften (1880), p. 136-139.

Velho, L.F.M., Lansac-Toha, F.A., Serafim, M.Jr., 1996, Testate amoebae 
(Rhizopodea-Sarcodina) from zooplankton of the high Parana River floodp lain, state of Mato Grosso do Sul, Brazil: 1. Families Arcellidae and Centropyxidae: STUD.-

NEOTROP.-FAUNA-ENVIRON, v. 31, pp. 35-50

Verworn, M., 1888, Biologische Protisten-Studien: Zeitschrift für Wissenschaftliche Zoologie, v. 46, no 4, p. 455-470, pl. 32.

Verworn, M., 1889, Psycho-physiologische Protisten-Studien. Experimentelle Untersuchungen: p. I-VIII, 1-218, pls. 1-6; Gustav Fischer (Jena).

Verworn, M., 1890, Biologische Protisten-Studien. II: Zeitschrift für

Wissenschaftliche Zoologie, v. 50, no 2, p. 443-468, pl. 18.

Verworn, M., 1913, Irritability. A physiological analysis of the general effect of stimuli in living substance: $p$. I-XII, 1-264; Yale University Press, "Silliman Memorial Lectures" (New Haven).

Virieux, J., 1916, Recherches sur le plankton des lacs du Jura Central: Annales de Biologie Lacustre, v. 8, p. 5-192.

Voeltz-Höhn, J., 1971, Ein Beitrag zur Testaceenfauna (Protozoa, Rhizopoda ) des Hochmoores im Naturpark "Hoher Vogelsberg": Philippia (Kassel), v. I, p. 80-84.

Voeltzkow, A., 1891, Vorläufiger Bericht über die Ergebnisse einer Untersuchung der Süsswasserfauna Madagascars: Zoologischer Anzeiger, v. 14, p. 214-217, 221-230.

Volis, S.I., 1990,: Novye dlia fauny SSSR vidy kornenozhek Rhizopoda, Testacea v Kanevskom vodokhranilishche [New records of Rhizopoda, Testacea in Kanev Reservoir]: VESTN.-ZOOL., no. 2, p. 86

Volz, P., 1929, Studien zur Biologie der bodenbewohnenden Thekamöben: Archiv für Protistenkunde, v. 68, p. 349-408, pl. 6.

Volz, P., 1934, Untersuchungen über Mikroschichtung der Fauna von Waldboden: Zoologische Jahrbuch (Systematik), v. 66, p. 153-210.

Volz, P., 1951, Untersuchungen über Mikrofauna des Walbodens: Zoologische Jahrbuch (Systematik), v. 79, p. 514-566.

Volz, P., 1964, Über die soziologische und die physiognomische Forschungsrichtung in der Bodenzoologie: Verh. deutsch. zool. Gesellsch. p.522-532.

Vucetich, M. C., 1970, Algunos tecamebianos de la provincia de Formosa: Neotropica, v. 16, p. $42-48$.

Vucetich, M. C., 1972a, Tres nuevas tecamebas muscicoles para la Argentina (Rhizopoda, Testacea): Neotropica, v. 18, p. 126-128.

Vucetich, M. C., 1973a, Tecamebas muscicoles de la selva marginal de Punta Lara 
(Provincia de Buenos Aires, Argentina): Physis. Seccion B. Las Aguas Continentales y sus Organismos, v. 32, p. 61-66.

Vucetich, M. C., 1973b, Estudio de tecamebianos argentinos, en especial los del Dominion Pampasico: Rev. Mus. LaPlata, Sección Zoología, v. 11, no 108, p. 287332, 10 plates.

Vucetich, M. C., 1973c, Contribución al conocimiento de la ecologia y zoogeografia de los tecamebianos argentinos: Rev. Mus. LaPlata, Sección Zoología, v. 11, no 109, p. 333-358.

Vucetich, M. C., 1973d, Thecamoebans of General Vintter and Puelo lakes, Chubut, Argentina: Neotropica, v. 18, no 58, p. 1-10.

Vucetich, M. C., 1975a, Tecamebianos muscícolas y esfagnícolas de islas Malvinas (Argentina): Neotropica, v. 21, no 64, p. II-16.

Vucetich, M. C., 1975b, Tecamebianos de mallines y otros ambientes lénticos de la Patagonia andina (Rhizopoda, Testacea): Neotropica, v. 21, no 65, p. 104-112.

Vucetich, M. C., 1978, Nuevos aportes al conocimiento de los tecamebianos del Dominio Subtropical: Neotropica, v. 24, p. 79-90.

Vucetich, M.C., 1973e, Una nueva especie del genero Nebela Leidy (Rhizopoda, Testacea): Neotropica, v. 19, pp. 79-81.

Vucetich, M.C., 1976, Tecamebianos del Lago San Roque y de un ambiente vinculado al mismo (Cordoba, Argentina): Limnobios, v. 1, pp. 29-34

Vucetich, M.C., 1989, Descripcion y ultrastructura tecal de dos especies nuevas del genero Difflugia Leclerc: Limnobios, v. 2, pp. 740-743

Vucetich. M. C., 1972b, Tecamebianos del eupleuston de cuerpos de agua de la Provincia de Buenos Aires: Acta Zoologica Lilloana (Tucuman, Argentina), v. 29, p. 271-284.

Wagner, R., 1834-1835, Lehrbuch der vergleichenden Anatomie: p. I-XXX, 1-607; Leopold Voss (Leipzig). - thecamoebians (?).

Wagner, R., 1841, Icones Zootomicae. Handatlas zur vergleichenden Anatomie nach fremden und eigenem Untersuchungen Zusammengestellt: $\mathrm{p}$. I-XVI, 1-24, pls. 1-35; Leopold Voss (Leipzig).

Wailes, G. H., 1912, Identification of Rhizopoda from Port Erin: Transactions of the Liverpool Biological Society, v. 26, p. 20.

Wailes, G. H., 1912a, Freshwater Rhizopoda from the Hebrides, Orkney and Shetland Islands, and Western Scotland; with description of a new species: Scottish Naturalist, 1912 , p. 59-65, 141-142. 
Wailes, G. H., 1912b, Freshwater Rhizopoda and Heliozoa from the States of New York, New Jersey, and Georgia, U.S.A.; with supplemental note on Seychelles species: Journal of the Linnean Society of London, Zoology, v. 32, no 214, p. 121-161, pl. 12.

Wailes, G. H., 1913a, Freshwater Rhizopoda from North and South America: Journal of the Linnean Society of London, Zoology, v. 32, no 216, p. 201-218, pl. 15.

Wailes, G. H., 1913b, Freshwater Rhizopoda from the North and West Ridings of Yorkshire: Naturalist (Hull, London), p. 144-148.

Wailes, G. H., 1913b, Freshwater Rhizopoda: in: Murray, J. and Wailes, G. H., "Notes on the natural History of Bolivia and Peru", p. 31-40, 44-45; The Scottish Oceanographic Laboratory (Edinburgh).

Wailes, G. H., 1915a, Rhizopoda, Part III: in: Cash, J., Wailes, G. H. and Hopkinson, J., "The British freshwater Rhizopoda and Heliozoa", v. III, p. I-XXIV, 1-156, pls. 33-57, Ray Society Publication no 98; Ray Society (London).

Wailes, G. H., 1915b, Notes on the structure of tests of freshwater Rhizopoda: Journal of the Royal Microscopical Society, p. 105-116, pls. 2,3.

Wailes, G. H., 1919, Supplement to the Rhizopoda in: Cash, J.,Wailes, G. H., and Hopkinson, J., "The British freshwater Rhizopoda and Heliozoa", v. IV, p. VIII-XII, 1716, pls. 58-63, Ray Society Publication no 103; Ray Society (London). ??is this under Cash Wailes, G. H., 1925, Some new or rare Protozoa from British Columbia: Annals and Magazine of Natural History, ser. 9, v. 16, no 91, p. 40-48, pls. 1-2.

Wailes, G. H., 1927, Rhizopoda and Heliozoa from British Columbia: Annals and Magazine of Natural History, ser. 9, v. 20, no 115, p. 153-156.

Wailes, G. H., 1928, Freshwater and marine Protozoa from British Columbia, with description of new species: Art, Historical and Scientific Association of Vancouver, B. C.: Museum and Art Notes, v. 3, no 3, p. 25-37, pls. 7-9; no 4, p. 28-31, pls. 11-12.

Wailes, G. H., 1930a, Some Algae and Protozoa from Como Lake: Art,Historical and Scientific Association of Vancouver, B. C.: Museum and Art Notes, v. 5, no 1, p. 26-27.

Wailes, G. H., 1930b, Munday Lake ecology, Part I: Art, Historical and Scientific Association of Vancouver, B. C.: Museum and Art Notes, v. 5, no 3, p. (?)

Wailes, G. H., 1930c, Protozoa and Algae, Mount Ferguson, B. C.: Art, Historical and Scientific Association of Vancouver, B. C.: Museum and Art Notes, v. 5, no 4, p. 160-165.

Wailes, G. H., 1931, Protozoa and Algae, Mount Ferguson, B. C. Part II: Art, Historical and Scientific Association of Vancouver, B. C.: Museum and Art Notes, v. 6, no 2, p. $72-75$. 
Wailes, G. H., 1931a, Munday Lake and its ecology. Part II: Art, Historical and Scientific Association of Vancouver, B.C.: v. 6, no 1, p. 34-37.

Wailes, G. H., 1933, Subalpine Protozoa and Algae from the Tulameen \& Garibaldi areas, B. C.: Art, Historical and Scientific Association of Vancouver, B. C.: Museum and Art Notes, v. 8, no 1, p. 1-3.

Wailes, G. H., 1934, Freshwater Algae and Protozoa from Alaska, with records of Cladocera and Rotifera. Part II: Art, Historical and Scientific Association of Vancouver, B. C.: Museum and Art Notes, v. 9 (Suppliment no 10), p. I, 1-4.

Wailes, G. H., Penard, E., 1911, Rhizopoda: Proceedings of the Royal Irish Academy, v. 31, "A biological survey of Clare Island in the County of Mayo, Ireland, and of the adjoining district", sect. 3, "Zoology (Oligochaeta to Protozoa). Marine Ecology. Summary", Part 65, p. 1-64, pls. I-VI.

Wailes, G.H. and Penard, E. (1911) Clare Island Survey: Rhizopoda. Proceedings of the Royal Irish Academy 31 (65) 1-64. 


\section{WAL-ZYK}

Wallich, G. C., 1862, The North Atlantic Sea-Bed; comprising a diary of the voyage on board H. M. S. Bulldog, in 1860, and observations on the presence of animal life, and the formation and nature of organic deposits, at great depths in the ocean, Part I: p. I-II, 1-160, 6 plates; J. Van Voorst (London).

Wallich, G. C., 1863a, Further observations on an undescribed indigenous Amoeba, with notices on remarkable forms of Actinophrys and Difflugia: Annales and Magazine of Natural History, ser. 3, v. 11, no 65, p. 365-371, pl. 9.

Wallich, G. C., 1863b, Further observations on Amoeba villosa and other indigenous Rhizopods: Annals and Magazine of Natural History, ser. 3, v. 11, no 66, p. 434-453, pl. 10.

Wallich, G. C., 1863c, On the value of the distinctive characters in Amoeba: Annals and Magazine of Natural History, ser. 3, v. 12, no 68, p. 111-151.

Wallich, G. C., 1863d, Further observations on the distinctive characters and reproductive phenomena of the amoeban Rhizopods: Annals and Magazine of Natural History, ser. 3, v. 12, no 71, p. 329-337.

Wallich, G. C., 1863e, Further observations on the distinctive characters, habits, and reproductive phenomena of the amoeban Rhizopods: Annals and Magazine of Natural History, ser. 3, v. 12, no 72, p. 448-468, pl. 8.

Wallich, G. C., 1864a, On the process of mineral deposit in the rhizopods and sponges, as affording a distinctive character: Annals and Magazine of Natural History, ser. 3, v. 13 , no 73 , p. $72-82$.

Wallich, G. C., 1864b, On the extent, and some of the principal causes, of structural variation among the Difflugian Rhizopods: Annals and Magazine of Natural History, ser. 3, v. 13, p. 215-245, pls. 15,16.

Wallich, G. C., 1865 , On the structure and affinities of the Polycystina: Quarterly Journal of Microscopical Science, v. 13 (=new series, v. 5), p. 57-84.

Wallich, G. C., 1869 , On the rhizopods as embodying the primordial type of animal life: Monthly Microscopical Journal, v. (?), p. 228-235.

Wallich, G. C., 1875, The amoeban, actinophryan and difflugian rhizopods: Monthly Microscopical Journal, v. 13, p. (?)-210.

Wallich, G. C., 1877a, On the fundamental error of constituting Gromia the type of foraminiferal structure: Annals and Magazine of Natural History, ser. 4, v. 19, no 110, p. $158-174$.

Wallich, G. C., 1877b, Observations on the Coccosphere: Annals and Magazine of 
Natural History, ser. 4, v. 19, no 113, p. 343-350, pl. 17.

Wallich, G. C., 1885, Critical observations on Prof. Leidy's "Freshwater Rhizopods of North America", and classification of the Rhizopods in general: Annals and Magazine of Natural History, ser. 5, v. 16, no 95, p. 317-334; v. 16, no 96, p. 453-473.

Wang, J., 1977, English summary: Protozoa from some districts of Tibetan Plateau: Acta Zoologica Sinica, [original text in chinese] v. 23, no 2, p. 131-160.

Wang, P.X, Min, Q.B., 1987, On the paleoenvironmental significance of thecamoebians in Quaternary deposits of China, Acta Micropalaeontologica Sinica [Weiti Gushengwu Xuebao], v. 4, pp. 345-349

Wanner, M., 1995, Biometrical investigations of terrestrial testate amoebae (Protozoa: Rhizopoda) as a method for bioindication: ACTA-ZOOL.-FENN., no. 196, pp. 267-270

Wanner, M., Esser, S., Meisterfeld, R., 1994, Effects of light, temperature, fertilizers and pesticides on growth of the common freshwater and soil species Cyclopyxis kahli (Rhizopoda, Testacealobosia), interactions and adaptations: Limnologica, v. 24, no. 3, pp. 239-280

Wanner, M., Meisterfeld, R., 1994, Effects of some environmental factors on the shell morphology of testate amoebae (Rhizopoda, Protozoa): European Journal of Protistology, v. 30, pp. 191-195

Waringer, J.L., 1996,: Cretaceous marginal marine and freshwater rhizopods from central Nova Scotia, Canada: Unpublished B.Sc. honors thesis, Dalhousie University, Halifax, N.S., Canada.

Warner, B.G. (1987) Abundance and diversity of testate amoebae (Rhizopoda, Testacea) in Sphagnum peatlands in southwestern Ontario, Canada. Archiv für Protistenkunde, 133 173-189.

Warner, B.G. (1990) Testate Amoebae (Protozoa). Methods in Quaternary ecology. Geoscience, Canada 5. Geological Association of Canada. 65-74.

Warner, B.G. (1991) Fossil testate amoebae (Protozoa) and hydrological history of an ombrotrophic bog in northwestern Ontario, Canada. In Spigarelli, S.A. (ed.)

Proceedings of an International Symposium on Peat/Peatland Characteristics and Uses. Minnesota, Bemidji State University, 5-14.

Warner, B.G., Charman,-D.J., 1994, Holocene changes on a peatland in northwestern Ontario interpreted from testate amoebae (Protozoa) analysis: Boreas, v. 23, no. 3, pp. 270-279

Warner, B.G., Chmielewski, J.G., 1992, Testate amoebae (Protozoa) as indicators of drainage in a forested mire, Northern Ontario, Canada: Archiv für Protistenkunde, $v$. 141 , pp. $179-183$ 
West, G. S., 1901, On some British freshwater Rhizopods and Heliozoa: Journal of the Linnean Society of London, Zoology, v. 28, no 183, p. 308-342, pls. 28-30.

West, G. S., 1903, Observations on freshwater Rhizopods with some remarks on their classification: Journal of the Linnean Society of London, Zoology, v. 29, p. 108-117, pl. 13.

West, G. S., 1905, Notes on some Scottish freshwater Rhizopoda and Heliozoa. I. Rhizopods from the Orkneys and Shetlands: Annals of Scottish Natural History, p. 89-94.

Wetzel, R. G., 1975, Limnology: p. I-XII, 1-743; W. B. Saunders Company (Philadelphia).

Whitelegge, T., 1886, List of the freshwater Rhizopoda of N. S. Wales. Part I. Proceedings of the Linnean Society of New South Wales, ser. 2, v. 1, no 2, p. 497504.

Whitelegge, T., 1889, List of the marine and freshwater invertebrate fauna of Port Jackson and neighbourhood: Journal of the Royal Society of New South Wales, v. 23, no 2, p. 163-323.

Wiesner, H., 1931, Die Foraminiferen der deutschen Südpolar-Expedition 1901-1903: in: Drygalski, E. von, (ed.), "Deutsche Südpolar-Expedition 1901-1903, im Auftrage des Reichsministeriums des Innern": Band 20 (= Zoologie, Band 12), p. 49-165, pls. 1-24; Walter DeGruyter \& Co. (Berlin and Leipzig).

Wightman, W.G., Scott, D.B, Kvale, E.P., Archer,A.W., Feldman, H.W , 1993, Carboniferous agglutinated foraminifera and arcellaceans from coal-bearing strata; a new method of investigating environments of coal accumulation: In: Carboniferous to Jurassic Pangea, first international symposium, Beauchamp-B., Embry, E. , Glass, D.(eds.), Canadian Society of Petroleum Geologists. Calgary, Canada.

Wightman, W.G., Scott, D.B., Medioli, F.S, Gibling, M.R., 1992, Agglutinated foraminifera from the Sydney Coalfield, Nova Scotia, their use as indicators of sea-level changes in Carboniferous coal-bearing strata: Abstract, Geological Society of America, v. 24, pp. 226.

Wightman, W.G., Scott, D.B; Medioli, F.S., Gibling, M.R , 1994, Agglutinated foraminifera and thecamoebians from the Late Carboniferous Sydney Coalfield, Nova Scotia: Paleoecology, Paleoenvironments and Paleogeographical implications; In: The Euroamerican coal province; controls on tropical peat accumulation in the Paleozoic; Calder, J.H., Gibling, M.R.(eds.); Palaeogeography, Palaeoclimatology, Palaeoecology, v. 106, pp. 187-202, Elsevier, Amsterdam, Netherlands.

Wilkinson, D.M., 1990, Glacial refugia in South Georgia? Protozoan evidence: Quaternary Newsletter, v. 62, pp. 12-13. 
Wilkinson, D.M., 1990, Multivariate analysis of the biogeography of the protozoan genus Nebela in southern temperate and Antarctic zones: European Journal of Protistology, v. 26, pp. 117-121.

Wilkinson, D.M. (1994) A review of the biogeography of the protozoan genus Nebela in the southern temperate and Antarctic zones. Area, 26 150-157.

Williamson, W. C., 1858, On the Recent Foraminifera of Great Britain: p. I-XX, 1107, 7 plates; Ray Society (London).

Wohlam, A., Allen, R.D., 1968, Structural organization associated with pseudopod extension and contraction during cell locomotion in Difflugia: Journal of Cellular Science, v. 3, p. 105-114.

WohIman, A., Allen, R.D., 1968, Structural organization associated with pseudopod extension and contraction during cell locomotion in Difflugia.: Journal of Cell Science, v. 3, pp. $105-114$.

Wolf, M., 1995, Verkieste Amoeben in Steinkohlen aus dem Ruhrgebiet, erster Nachweis von Arcella Ehrenberg im Palaeozoikum: Palaeontologische Zeitschrift, v. 69, pp. 1-6.

Wolff, H., 1948, Hydrobiologische Untersuchungen an den hochalpin seen des San Bernardinopasses: Revue für Hydrobiologie, v. 10, p. 101-144.

Woodland, W.A., Charman, D.J. and Sims, P.C. (1998) Quantitative estimates of water tables and soil moisture in Holocene peatlands from testate amoebae. The Holocene, 8 261-273.

Wright, E. P., 1867, Remarks on freshwater rhizopods: Quarterly Journal of Microscopical Science, new ser., v. 7, p. 174-175. Reprinted as: Wright, 1868.

Wright, E. P., 1868, Remarks on freshwater rhizopods: Proceedings of the Dublin Microscopical Club, v. 1, no 2, p. 118-119. Reprinted from: Wright 1867.

Wright, E. P., 1870, No title: Quarterly Journal of Microscopical Science, new ser., v. 10, p. 305.

Wright, T. S., 1861a, On the reproductive elements of the Rhizopoda: Annals and Magazine of Natural History, ser. 3, v. 7, no 41, p. 360-362, pl. 18.

Wright, T. S., 1861b, Observations on British Protozoa and Zoophytes: Annals and Magazine of Natural History, ser. 3, v. 8, no 44, p. 120-135, pls. 3-5.

Zacharias, 0., 1889, Bericht über eine zoologische Exkursion an die Kraterseen der Eifel: Biologisches Centralblatt, v. 19, p. 56-64, 76-80, 107-113.

Zacharias, 0., 1890, Zur Kenntnis der Tierwelt des Reisengebirges nebst vergleichenden Ausblicken: Forschungsberichte deutsch. Land. Volksk. v. 4, p. 399- 
433.

Zacharias, 0., 1891, Die Fauna des Süsswassers in ihren Beziehungen zu der des Meeres: in: Zacharias, O., "Die Tier und Pflanzenwelb des Susswasser", v. I, p. 297312.

Zacharias, 0., 1893, Faunistische und Biologische Beobachtungen am Grossen PIner: Forschungsberichte aus der Biologischen Station zu Plön, v. I, 52 p.

Zacharias, 0., 1894, Faunistische Mitteilungen: Forschunsberichte aus der Biologischen Station zu Plön, v. 2, p. 57-59, pls. 1-2.

Zacharias, 0., 1897a, Neue Beiträge Kenntnis des Süsswasserplanktons:

Forschungsberichte aus der Biologischen Station zu Plön, v. 5, p. 1-9, pl. I.

Zacharias, 0., 1897b, Zur Mikrofauna der Sandforten Taiche: Forschungsberichte aus der Biologischen Station zu Plön, v. 5, p. 112-114.

Zacharias, 0., 1899, Die Rhizopoden und Heliozoen des Süsswasserplanktons:

Zoologischer Anzeiger, v. 22, p. 49-53.

Zacharias, 0., 1902, Zur Flora und Fauna der Schilfstengel im Grossen Plner:

Forschungsberichte aus der Biologischen Station zu Plön, v. 9, p. 17-25.

Zacharias, 0., 1903, Zur kenntnis der niedern Flora und Fauna Holsteinischer Moorstümpfe: Forschungberichte aus der Biologischen Station zu Plön, v. 10, p. 223-289, pl. 2.

Zapata, A., Rudolph, E., 1981, Distribucion de foraminiferos y tecamebas en el estuario del Rio Contaco, Osorno: ARCH.-BIOL.-MED.-EXP., v. 14, p. 304

Zapata, J., Crespo, J., 1990, Tecamebas del volcan Rano-Kau, Isla du Pascua (27ํㅜㄴ $10^{\prime}$ S, $\left.109^{\circ} 26^{\prime} \mathrm{W}\right)$ : Biota, v. 6, pp. 53-59

Zapata, M. A., Rudolph, L.E., 1986, Tecamebas del rio Damas: Biota, v. 2, pp. 65-82

Zarnik, B., 1908, Über eine neue Ordnung der Protozoen: Sitzungsberichte der Physikalisch-medicinischen Gesellschaft zu Würzburg, p. 72-78.

Ziegler, H. E., 1907, Zoologisches Wörterbuch. I, Lieferung, A-F: P. ??368; (Jena). [what should be bold?]

Zimmer, C., 1899, Das tierische Plankton der Oder: Das Plankton der Oderstrome; Forschungsberichte aus der Biologischen Station zu Plön, v. 7. p. 1-14.

Zivkovi, A., 1975a, Nouvelles et rares espèces de Testacea (Rhizopoda) dans la fauna (sic) de (sic) Danube: Glasnik Prirodnjackog Muzeja, Beograd, Serija B, Bioloske Nauke/ Bulletin du Muséum d'Histoire Naturelle, Belgrade, Série B, Sciences Biologiques, v. 30, p. 119-123, pl. 1. 
Zivkovi, A., 1975b, Contribution à la connaissance des espèces de (sic) genre Protocucurbitella Gauthier-Livre (sic) et Thomas dans la faune de Yougoslavie: Glasnik Prirodnjackog Muzeja, Beograd, Serija B, Bioloske Nauke/ Bulletin du Muséum d'Histoire Naturelle, Belgrade, Série B, Sciences Biologiques, v. 30, p. 147-152, pl. 1.

Zschokke, F., 1900, Die Thierwelt in den Hochgebirgsseen: Neue Denkschriften des Allgemeine Schweizeriche Gesellschaft für gesamten Naturwissenschaften, v. 37, p. I-IV, 1-400, pls. 1-8.

Zschokke, F., 1907, Ubersicht über die Tiefenfauna des Vierwaldstättersees: Archiv für Hydrobiologie, v. 2, no 1, p. 1-8.

Zschokke, F., 1908a, Die Resultate der Zoologischen Erforschumg hochalpner Wasserbecken seit dem Jahre 1900: Internationale Revue der gesamten Hydrobiologie und Hydrographie, v. I, p. 221-235.

Zschokke, F., 1908b, Beziehungen zwischen Tiefenfauna subalpiner seen und der Tierwalt von kleingewässern des Hochgebirges: Internationale Revue der gesmten Hydrobiologie und Hydrographie, v. I, p. 783-790.

Zschokke, F., 1912, Leben in der Tiefe subalpiner seen, uberreste der eiszeitlichen Mischfauna: Archiv für Hydrobiologie, v. 8, p. 109.

Zuelzer, M., 1904, Beiträge zur Kenntnis von Difflugia urceolata Carter: Archiv für Protistenkunde, v. 4, no 2, p. 240-295, pls. 10-12.

Zune, A. J., 1894, Traito d'analyse chimique, micrographique, et micro-biologique des eaux potables: Paris - no further information available.

Zykoff, W., 1904, Das Plankton des Seliger Sees: Zoologischer Anzeiger, v. 27, p. 388-394.

Zykoff, W., 1906, Über das Plankton des Saisan-Sees: Zoologischer Anzeiger, v. 29, p. $477-482$. 